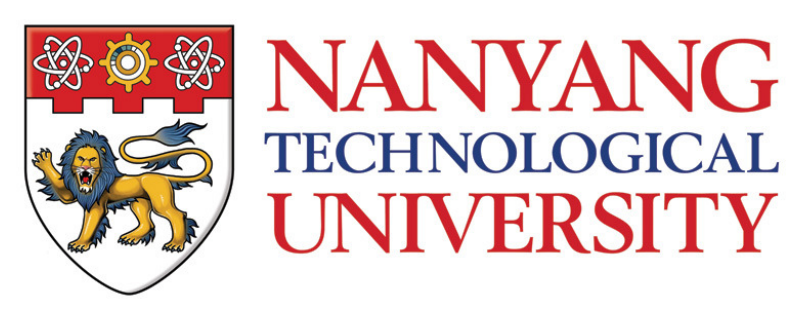

\title{
SURFACE ACOUSTIC WAVE (SAW) DESIGN AND APPLICATIONS IN MICROFLUIDICS
}

\author{
RAHUL KISHOR \\ INTERDISCIPLINARY GRADUATE SCHOOL \\ NANYANG ENVIRONMENT \& WATER RESEARCH INSTITUTE (NEWRI)
}




\title{
SURFACE ACOUSTIC WAVE (SAW) DESIGN AND APPLICATIONS IN MICROFLUIDICS
}

\author{
RAHUL KISHOR
}

INTERDISCIPLINARY GRADUATE SCHOOL

NANYANG ENVIRONMENT \& WATER RESEARCH INSTITUTE (NEWRI)

A Thesis Submitted to the Nanyang Technological University in partial fulfilment of the requirement for the degree of Doctor of Philosophy 



\section{Acknowledgements}

With great pleasure I would like to thank my thesis advisor Prof Zheng Yuanjin for his support and guidance provided to me during my stint at NTU as a PhD student. I am deeply indebted to you for the faith and confidence bestowed on me over these years. My heartfelt thanks to Prof Lim Teik Thye and Dr Wang Zhenfeng, my cosupervisor and Prof Richard Webster, my mentor for their invaluable suggestions and recommendations.

The completion of this highly interdisciplinary undertaking could not have been possible without the assistance of my teammates. I would like to especially thank Dr Feng Xiaohua, Dr Ding Ran, Dr Gao Fei, Dr Zhu Yao and Liu Siyu for their help and support. A special gratitude to Seah Yen Peng Daphne for her guidance and unwavering support. Dr Sivaramapanicker Sreejith, thanks for your extreme patience in taking my thoughts and making them look highly professional with your amazing skills. The days in NTU was enjoyable from the friends I made over these years. Thank you all for being with me throughout these years.

I thank NTU and NEWRI for awarding me the postgraduate scholarship to support my studies.

This journey would have been difficult without the backing of my in-laws and relatives. To my sister Heera, you deserve my whole-hearted thanks for being there 
always to encourage me. To my father, who is always in my thoughts- you are missed. To my mother, Padmaja Kishor, who was my first teacher and taught me the value of hard work and education. Thank you for your unwavering love and faith in me.

Words are not enough to express my love and gratitude to my beloved wife, Sreedevi.You have been with me throughout this journey, and I couldn't complete this without your selfless support, sacrifices and patience. This thesis is dedicated to you. 


\section{Abstract}

Lab-on-a-chip (LOC) is the current trend towards developing point-of-care devices. LOC finds its application in fields of medicine, environmental monitoring and towards a multitude of industrial applications. It involves integrating all the functions that were done in a laboratory, from input samples to results delivery in an easy-to-handle device. This was realised due to the advent of microfluidics and the microelectronics technology. Physically, an LOC consists of four main parts: microfluidics, actuators, sensors and readout circuits. Many research groups along with startup companies have developed technologies to realise fluid actuation/control and signal detection with impressive capabilities. However, there is a dearth of LOC's available in the market. They are still confined within the spaces of the laboratory and regarded as a "chip-onlab" functionality, justifiably due to the lack of an integrated platform that performs the different assay procedures in a seamless and automated fashion. This work is in pursuit of developing an integrated platform to realise an LOC utilising surface acoustic wave (SAW) devices.

SAW are nanometer amplitude vibrations that are generated on a piezoelectric substrate. During the last decade, SAW has been intensively used and researched for microfluidic applications majorly as an actuator. SAW capabilities as a sensor for immunoassay was also explored. This unique feature of SAW to act both as an actuator and sensor makes it easier for integration. In this thesis, we first study the use of 
SAW as an actuator of the fluids by establishing a novel mechanism for characterising the SAW energy transmission in fluidic channels, which is essential for all the SAW microfluidics design, using a mixing structure. We developed analytical models in this work that could be used to optimise the power transmission coefficient and hence increase actuation efficiency.

Besides focusing on the application of SAW as an actuator, its usage as a sensor was primarily relying on the immunoassay technique which required complicated surface preparation steps. In the subsequent work, we proposed and demonstrated a new sensing methodology utilising photoacoustic induced surface acoustic wave (SAW-PA) for simultaneous optical and mechanical property characterization of analytes (including cells, nanoparticle and dyes). A nanosecond pulsed laser excitation on a sample triggers a longitudinal acoustic wave in the fluid which is modeconverted into a Rayleigh SAW on the piezoelectric substrate and detected using the metal electrodes (interdigital transducer, IDT). We further developed a numerical model to study the wave conversion process (longitudinal acoustic waves to SAW) and demonstrate that the PA generated in the microfluidic channel acts as a mechanical resonator dependent on the dimensions of the microfluidic channel. We experimentally verified that a SAW device matched to the channel resonant frequency could improve the sensitivity.

Finally, we propose a platform combining the SAW actuators and SAW-PA sensor, which is closer to realising the initial objective of an integrated platform for LOC. In this work, we have developed an integrated microfluidic system that combined high-efficiency (> 95\%) tilted-angle standing surface acoustic wave (taSSAW) based particle separation, particle concentration inside an open microfluidic chamber and sensing, on a single $\mathrm{LiNbO}_{3}$ substrate. The platform demonstrated real-time quantitative detection of $10 \mu \mathrm{m}$ polystyrene beads down to 7 particles in $10 \mu \mathrm{l}$ of the sample volume in 15 minutes. 


\section{Contents}

Acknowledgements $\ldots \ldots \ldots \ldots \ldots \ldots \ldots \ldots \ldots \ldots$

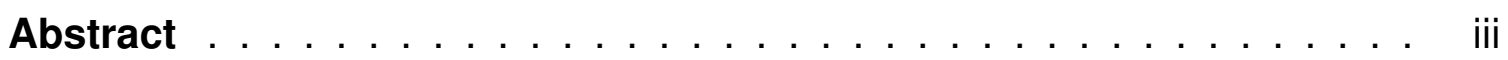

List of Figures $\ldots \ldots \ldots \ldots \ldots \ldots \ldots \ldots \ldots \ldots \ldots \ldots \ldots \ldots$

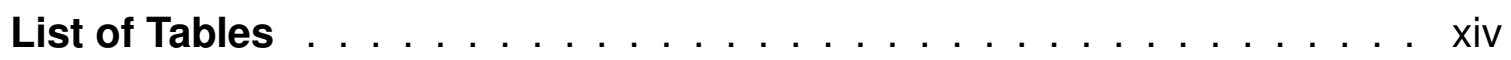

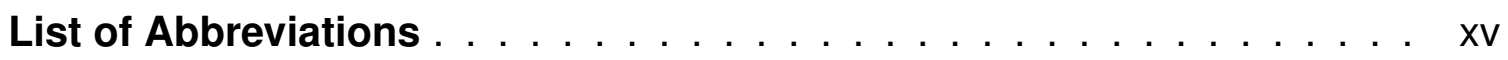

1 Introduction 1

1.1 Motivation for this thesis $\ldots \ldots \ldots \ldots \ldots \ldots$

1.2 Objectives ..................... 3

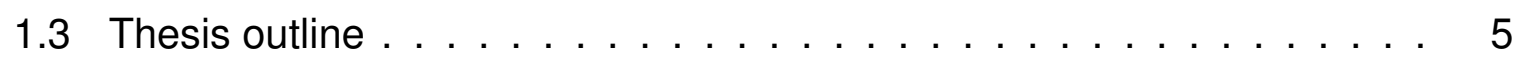

2 Literature Review $\quad 7$

2.1 Defining Lab-on-a-chip . . . . . . . . . . . . . . . 7

2.2 Processes involved in an LOC $\ldots \ldots \ldots \ldots \ldots$ 
2.2.1 Sample handling . . . . . . . . . . . . . . . . 8

2.2 Separation . . . . . . . . . . . . . . . . . 12

2.2 .3 Detection . . . . . . . . . . . . . . . . . . 13

2.3 Surface acoustic wave microfluidics . . . . . . . . . . . . . . . . 18

2.3.1 SAW fluid actuation and manipulation . . . . . . . . . . . . 22

3 Acoustofluidic mixing: The study of an acoustically coupled multi-layered microfluidic platform on SAW substrate $\quad 25$

3.1 Introduction . . . . . . . . . . . . . . . 26

3.2 Materials and methods . . . . . . . . . . . . . . . . 28

3.2.1 SAW device design and simulation . . . . . . . . . . 28

3.2.2 Micromixer design $\ldots \ldots \ldots \ldots \ldots$

3.3 Experimental setup . . . . . . . . . . . . . . . . 31

3.3.1 Electrical setup . . . . . . . . . . . . . . . . . 31

3.3.2 Coupling layer $\ldots \ldots \ldots \ldots \ldots \ldots \ldots \ldots$

3.3.3 Placement of the microfluidic channel . . . . . . . . . . 33

3.3.4 Microfluidic setup . . . . . . . . . . . . . . . . . . . . . 33

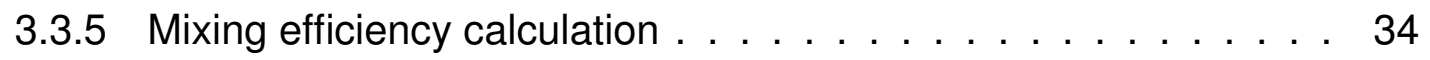

3.4 Results and discussion $\ldots \ldots \ldots \ldots \ldots \ldots$ 
3.4.1 Transient behaviour of mixing . . . . . . . . . . . 36

3.4.2 Effect of voltage on mixing . . . . . . . . . 37

3.4.3 Effect of coupling layer thickness on mixing efficiency $\ldots . . . .37$

3.4.4 Effect of frequency on mixing . . . . . . . . . . . . . 40

3.4 .5 Acoustic heating . . . . . . . . . . . . . . . 43

3.5 Concluding remarks $\ldots \ldots \ldots \ldots$

4 Photoacoustic induced surface acoustic wave (SAW PA) sensor for sens$\begin{array}{ll}\text { ing analytes in a microfluidic channel } & 47\end{array}$

4.1 Introduction ......................... 48

4.2 Materials and Methods . . . . . . . . . . . . . . . . . . 50

4.2.1 SAW device design and fabrication . . . . . . . . . . . . . 50

4.2.2 Design of microfluidic channel . . . . . . . . . . . . . . 51

4.2.3 Reagent and solutions . . . . . . . . . . . . . . . . . . 51

4.2 .4 Experimental setup . . . . . . . . . . . . . . 52

4.2.5 Sensor theory and data processing . . . . . . . . . . . . . . . . 54

4.3 Experimental Results and discussion . . . . . . . . . . . . . . . 60

4.3.1 SAW-PA signal from standard dye solutions . . . . . . . . . . . 60

4.3.2 SAW-PA signals from gold nanoparticles . . . . . . . . . . . . . . 64 
4.4 FEM modelling of the SAW-PA sensor . . . . . . . . . . . . . . . . . 70

4.4.1 Theoretical analysis ................. . . 70

4.4 .2 Finite element mode . . . . . . . . . . . . . . . . . 71

4.4.3 Experimental measurement . . . . . . . . . . . . . . . 77

4.4.4 Sensitivity calculation . . . . . . . . . . . . . . 80

4.5 Conclusion . . . . . . . . . . . . . . . . . . . . 80

5 Integrated microfluidic system using SAW for real-time separation and detection of particles on a single substrate

5.1 Introduction . . . . . . . . . . . . . . . 83

5.2 Materials and methods . . . . . . . . . . . . . . . . . 85

5.2.1 Device design and operation . . . . . . . . . . . . 85

5.2.2 SAW device fabrication . . . . . . . . . . . . . 88

5.2.3 SAW device characterization . . . . . . . . . . . . . . 88

5.2.4 Functionality of the integrated device . . . . . . . . . . . 88

5.2 .5 Materials ........................ 96

5.3 Results and discussion . . . . . . . . . . . . . . . . . 96

5.3.1 Separation of particles . . . . . . . . . . . . . . . 96

5.3.2 Concentration of particles . . . . . . . . . . . . . . . 98 
5.3.3 Sensing of particles . . . . . . . . . . . . . . 100

5.4 Conclusion .......................... 106

6 Conclusions and Future Work 108

6.1 Conclusions . . . . . . . . . . . . . . . . . 108

6.2 Future work . . . . . . . . . . . . . . . . . . 110

$\begin{array}{ll}\text { Appendix A Lamb waves in a plate } & 112\end{array}$

A.1 Derivation of stress in an isotropic solid . . . . . . . . . . . . . . 114

A.2 Derivation of mechanical displacement components of Lamb wave . . . 115

Appendix B Transmission coefficient of a sound wave from an arbitrary number of layers

Bibliography 


\section{List of Figures}

2.1 Photoacoustic principle $\ldots \ldots \ldots \ldots \ldots \ldots \ldots$

2.2 Schematic of a surface acoustic wave device $\ldots \ldots \ldots$

2.3 Schematic of the SAW interacting with a droplet . . . . . . . . . . 21

3.1 Fabricated SAW devices at a centre frequency of $50 \mathrm{MHz} \ldots \ldots .29$

3.2 Measured $\mathrm{S} 11$ using the network analyser . . . . . . . . . 30

3.3 Reusable SAW mixing schematic . . . . . . . . . . . . . . . 31

3.4 PDMS as a reservoir to control coupling layer thickness $\ldots \ldots \ldots 32$

3.5 Microfluidic $Y$ channel placed on top of the substrate via coupling layer . 33

3.6 Y shaped microchannel on PDMS . . . . . . . . . . . . 34

3.7 Images of the SAW mixing experiment . . . . . . . . . 35

3.8 Transient mixing phenomena $\ldots \ldots \ldots \ldots \ldots$

3.9 Mixing efficiency variation with voltage $\ldots \ldots \ldots \ldots \ldots$

3.10 Acoustic wave transmission through the various layers $\ldots \ldots \ldots$. . . 39 
3.11 Mixing efficiency variation with coupling layer thickness . . . . . . . . . 40

3.12 Mixing efficiency variation with frequency $\ldots \ldots . . . . . . . . . .41$

3.13 Acoustic wave transmission with the wave refracting angles $\ldots$. . . . . 44

3.14 Setup for measuring the temperature on the SAW device $\ldots . . . . .45$

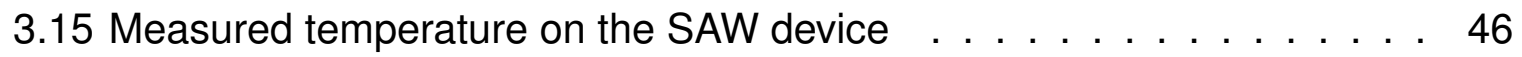

4.1 Frequency response (S11) of the SAW device . . . . . . . . . . . . . . 51

4.2 Schematic illustration of the PDMS microfluidic channel on the SAW substrate ........................... 52

4.3 Schematic representation of the SAW-PA device $\ldots . . \ldots 53$

4.4 Schematic of the experimental setup of the SAW-PA sensor . . . . . . 53

4.5 Photograph of SAW device with the bonded microfluidic channel . . . . 54

4.6 Schematic representation of the mode conversion process . . . . . . . . 55

4.7 Photoacoustic signal output obtained at the IDT . . . . . . . . . . 57

4.8 Background SAW-PA signal intensity generated by the PDMS microfluidic channel for different optical excitation wavelengths . . . . . . . . . 58

4.9 PSD spectra at different time durations . . . . . . . . . . . . . . . . 59

4.10 Repeatability of the SAW-PA signal . . . . . . . . . . . . . . . . 60

4.11 Measured absorption spectra of the dye . . . . . . . . . . . 61

4.12 SAW-PA response for different dye concentrations $\ldots . . . . . . . .63$ 
4.13 Effect of viscosity on SAW-PA response . . . . . . . . . . . . 65

4.14 SAW-PA response from gold nanoparticles (GNPs) . . . . . . . . . . . . 66

4.15 SAW response for different GNP sizes . . . . . . . . . . . . . . . . . 67

4.16 SAW-PA response of gold nanorods (GNRs) for different concentrations 68

4.17 UV-vis absorption spectra and TEM images of the gold nanostructures . 69

4.18 SAW-PA response dependence on shape of the nanoparticle $\ldots . . .71$

$4.192 \mathrm{D}$ geometry used for the SAW-PA sensor simulation . . . . . . . . . . . 72

4.20 Frequency spectra of the SAW-PA sensor . . . . . . . . . . . . . . . . 75

4.21 Influence of the microfluidic channel dimension on the frequency spectra 76

4.22 The experimental setup for measuring the SAW-PA sensors sensitivity to frequency . . . . . . . . . . . . . . . . . 78

4.23 Experimental results for the sensitivity measurement . . . . . . . . . . . 79

5.1 Schematic diagram of the SAW-PA integrated microfluidic device . . . . 86

5.2 Schematic of the experimental system used for SAW-PA sensing of particles . . . . . . . . . . . . . . . . . . . 87

5.3 Measured S-parameter for the different SAW IDTs on the integrated device ........................... 89

5.4 Design of the microfluidic channel for concentration stage . . . . . . . . 92

5.5 Sensitivity of the SAW-PA device to IDT centre frequency $\ldots$. . . . . . . 94 
5.6 Experimental results for the particle separation . . . . . . . . . . . . . . 97

5.7 Experimental results for the particle concentration . . . . . . . . . . . . 99

5.8 Experimental results to show the dependence of particle concentration on cavity size . . . . . . . . . . . . . . . . . . . . . . . . . . 99

5.9 2D Geometry used for the simulation with the corresponding domains . 102

5.10 Numerical simulation results for particle detection using SAW-PA sensor 103

5.11 Experimental results for the SAW-PA sensing of different particle concentrations . . . . . . . . . . . . . . . . 105

A.1 Isotropic Plate . . . . . . . . . . . . . . . . . . . . . . . . . . 112

B.1 Sound wave transmission through arbitrary number of layers . . . . . 120 


\section{List of Tables}

2.1 Various types of active micromixers $\ldots \ldots \ldots \ldots \ldots \ldots$

2.2 Comparison of active separation techniques found in existing lab-on-achip systems . . . . . . . . . . . . . . . . . . . . . . 14

2.3 Comparison of the detection techniques found in existing lab-on-a-chip systems . . . . . . . . . . . . . . . . 16

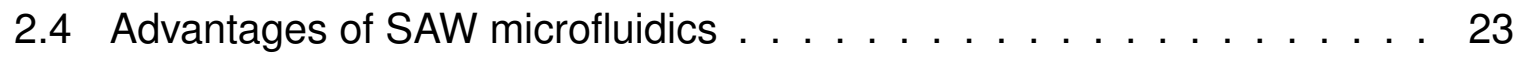

3.1 SAW design parameters $\ldots \ldots \ldots \ldots \ldots \ldots$

4.1 SAW-IDT design parameters $\ldots \ldots \ldots \ldots \ldots$

4.2 Thermoacoustic properties of water at $273^{\circ} \mathrm{C} \ldots \ldots \ldots \ldots$

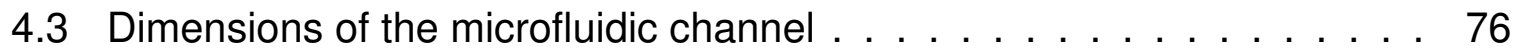




\section{List of Abbreviations}

$\begin{array}{ll}\text { IDT } & \text { Interdigital transducer } \\ \text { SAW } & \text { Surface acoustic wave } \\ \text { PA } & \text { Photoacoustics } \\ \text { LOC } & \text { Lab on a chip } \\ \text { taSSAW } & \text { Tilted-angle standing surface acoustic wave } \\ \text { LiNbO }_{3} & \text { Lithium niobate } \\ \text { PDMS } & \text { Polydimethylsiloxane } \\ \text { FEM } & \text { Finite element method } \\ \text { GNP } & \text { Gold nanoparticle } \\ \text { GNR } & \text { Gold nanorod }\end{array}$




\section{Chapter 1}

\section{Introduction}

A lab-on-a-chip (LOC) is a class of device which integrates the multitude of laboratory tasks into a single-chip, whose size varies from a few square millimetres to square centimetres. The whole purpose of an LOC is to perform an automated and a high throughput analysis of the samples for in situ/point-of-care diagnostics. The history of LOC is essentially linked to microfluidics, which is associated to the microtechnology used in semiconductors. The fabrication of MEMS devices in the early 60's subsequent to the micro technologies fostered the development of the first LOC- a gas chromatography, in 1979 by S.C. Terry at the Stanford University [1]. Subsequently, in the 80's there was a spur in the research towards LOC with the development of micropumps, sensors and also towards the production of polymer chips with ease in fabrication compared to the silicon technologies. In the 90's research exploration on microfluidics and miniaturisation of the biochemical process such as PCR by integrating various steps from sample collection to analysis, called generally as the Micro total analysis system ( $\mu$ Tas) [2]. Further, the improvement in individual components and its application to non-analysis lab processes inspired the term Lab-on-a-chip. Today for certain applications including chemical analysis [3], medical diagnostics [4] and en- 
vironmental monitoring [5], the LOC receives growing interests. The current trends in the research and product developments highlight that LOC will transform the diagnostics in the future. The challenge would be to integrate the maximum amount of functionalities to reduce cost, size and improve the speed of operation. Presently the technologies are not unified and hence impose the restriction on the ease of integration, research needs to address this point for their wide applicability [6,7]. As we explore in this thesis, certain technologies have the capability to perform a multitude of functionalities and therefore possess the potential to realise an LOC.

\subsection{Motivation for this thesis}

Microfluidics is the core component of any LOC system. It is the microscale conduit in an LOC. Whitesides [8] defines microfluidics as the "science and technology of systems that process or manipulate small $\left(10^{-9}\right.$ to $10^{-18}$ litres $)$ amount of fluids, using channels with dimensions of tens to hundreds of micrometres". Various features including reduced reagent and sample consumption, system miniaturisation and low cost become attractive in adhering to the requirements of an LOC. However, microfluidics possesses certain challenges, which limits a self-contained system, and requires integration with various active components to actuate and manipulate fluids. Some of the limitations include [9]:

- The increased surface area-volume ratio due to reduced dimensions causes the surface and viscous forces to dominate and reduce fluid flow.

- The Reynolds number in a microfluidic system where $\rho$ and $\mu$ are the density and the fluid viscosity, while $L$ and $v$ denotes the characteristic length and velocity, respectively. The low Re creates a laminar flow in the microfluidic channels and presents challenges in fluid mixing.

- Further, the application of LOC in bioanalysis requires the capability for addi- 
tional microfluidic manipulations including sample preparation steps such preconcentration and separation, with the ultimate goal of analyte detection.

The restrictions mentioned above emphasise the point that additional fluid control (actuator) units and sensors with microfluidics are essential to realise an LOC. Depending on the concerned applications, the components may vary. However, in general, a typical LOC would include sample handling, sample and reagent mixing, separation and finally detection $[6,10]$. In this thesis, we will discuss about using surface acoustic wave (SAW), which are nanomechanical waves propagating in a piezoelectric substrate, for microfluidic actuation and sensing and finally integrate them to actualize an LOC. Despite the current advancement in SAW microfluidics for actuation (to be discussed in detailed in Sec 2.3.1), their downstream detection still relies on using microscopes and cell counters like haemocytometers which are performed off-chip. The off-chip transferring involves the risk of sample loss, which is quite detrimental, especially for detection of rare specimens. However, a fully integrated LOC incorporating the SAW fluidic actuation with downstream detection is not realised.

\subsection{Objectives}

The broad scope of the thesis is to advance the state of art in SAW based LOC's by integrating SAW based- sensors and actuators with microfluidics on a single piezoelectric substrate, which has not been performed yet. Towards this goal, the thesis work focusses on the following:

(i) SAW actuator- There is a growing interest to couple SAW into disposable superstrates, which involves acoustic wave propagation through the various layer. Due to the multilayer propagation, the energy for fluid actuation is reduced.Coupling of SAW to superstrates was reported previously (details in Chapter 2) which did not address the effect of frequency and coupling layer thickness on the acoustic 
energy transmission efficiency. In this work, we perform a detailed characterization by proposing new analytical models to optimise the power transmission coefficient and experimentally demonstrated using the application of the fluid mixing phenomenon.

(ii) SAW sensor- Acoustic wave based sensors for evaluating mechanical parameters of fluid samples such as density, viscosity, etc. exists. However, devices for the simultaneous optical and mechanical characterization of fluids remains unexplored. Herein we propose a novel sensor combining photoacoustics (PA) and SAW to extract optical and mechanical characterization of fluids in micro volumes. The microfluidic channel acts as an acoustic resonator and numerical simulation using the finite element method (FEM) was used to explain the working principle.

(iii) SAW enabled LOC- Towards effectuating an integrated platform with sensors and actuators on the same chip, we designed and fabricated an integrated device which incorporates a SAW based separation technique and downstream detection on the same chip, moving closer to the expected goal of a true-LOC (as shown in chapter 5 ).

A common theme that runs through this thesis involves the study and optimisation of acoustic wave propagation: to impart energy into the fluid for actuation (discussed in chapter 3) and to receive the acoustic wave to sense the analyte properties (addressed in Chapter 4). From the SAW actuator (relying on acoustic streaming) and SAW sensor (photoacoustic induced SAW) studies in Chapter 3 and 4 respectively, we established the principles to finally incorporate a novel LOC based on SAW in Chapter 5. 


\subsection{Thesis outline}

Chapter 2: Literature Review.

In this chapter, we begin with a comprehensive introduction into the field of LOC by discussing the various processes involved in its realisation. We also review the various actuation and detection methods available in the microfluidic devices and stress on the significance of the acoustic based techniques. We then focus on an emerging optoacoustic sensing methodology- photoacoustics (PA) and its challenges in incorporating into microfluidics. Finally, an overview of the surface acoustic wave (SAW) based microfluidic actuation techniques is provided.

Chapter 3: Acoustofluidic mixing: The study of an acoustically coupled multi-layered microfluidic platform on SAW substrate.

In Chapter 3, we report a comprehensive study of SAW induced mixing on a reusable superstrate using extensive experiments and analysis. The study reveals a novel mechanism for characterising the SAW energy transmission in fluidic channels, using a mixing structure. The main contribution of the work comes from the discussion of the effect of thickness of coupling layer and frequency on the mixing efficiency in a Y-shaped microchannel and development of analytical models.

Chapter 4: Photoacoustic induced surface acoustic wave (SAW-PA) sensor for sensing analytes in a microfluidic channel

In this section, we introduce a novel sensor utilising the SAW IDTs to detect an acoustic signal generated by the optoacoustic resonance inside a microfluidic channel. We first demonstrate through experiments, the use of this SAW-PA platform for investigating the optical and acoustical properties of standard dye solutions and gold nanoparticles. We present analytical models to explain the acoustic mode conversion from the photoacoustic induced longitudinal waves to SAW.In this chapter, we explore the 
physics behind the SAW-PA sensing by performing a detailed numerical simulation using finite-element method. We show the existence of acoustic resonance inside the microfluidic channel and efficient conversion of a longitudinal wave generated inside the channel into SAW at eigen modes existing parallel to the piezoelectric substrate. We validate the numerical simulation by performing experiments to determine the sensitivity improvement of the SAW-PA sensor by choosing SAW IDTs with different centre frequencies.

Chapter 5: Integrated microfluidic system using SAW for real-time separation and detection of particles on a single substrate

This chapter binds the concepts of the SAW actuation and SAW-PA sensing to implement an integrated microfluidic platform. We combined the tilted angle standing surface acoustic wave based(taSSAW) separation, particle concentration and sensing on a single $\mathrm{LiNbO}_{3}$ substrate. The platform was used to demonstrate real-time quantitative detection of polystyrene beads. We first show high efficiency (> $95 \%$ ) separation of polystyrene beads $(3 \mu \mathrm{m}$ and $10 \mu \mathrm{m})$ using an SSAW. The separated particles were concentrated on an open PDMS cavity using a single SAW IDT. The microfluidic channel was designed to meet the conditions for the formation of a standing wave inside the fluid, to concentrate the particles. Experiments were conducted to prove the validity of the concept. The aggregated particles were further sensed using the SAW-PA sensor. The choice of optimum frequency was the design challenge, which was established using numerical modelling and experiments. Finally using experiments we demonstrate the performance of the sensor.

Chapter 6: Conclusions and future work

In the final part of the thesis, a summary of the work conducted will be presented with the list of key contributions. The chapter and thesis will conclude with potential future works. 


\section{Chapter 2}

\section{Literature Review}

In this chapter, a comprehensive introduction to the field of lab-on-a-chip (LOC) devices is presented by an overview of different steps involved in its realisation. Following, an overview of the two important functionalities in realising an LOC: actuation and sensing are discussed. A distinctive preference is given to acoustic based actuation methods by highlighting its advantages over other active techniques, which is the method to be considered in this thesis. A brief overview of the application of the different microfluidic actuation techniques by surface acoustic wave (SAW) is reviewed. A consideration of the various detection methods in microfluidics is summarised, with a focus on an emerging optoacoustic sensing methodology-photoacoustics (PA) and its challenges in incorporating into microfluidics.

\subsection{Defining Lab-on-a-chip}

A lab-on-a-chip (LOC) is a class of device which integrates the multitude of laboratory tasks into a single-chip, whose size varies from a few square millimetres to square centimetres. The whole purpose of an LOC is to perform an automated and a 
high throughput analysis of the samples for in situ/point-of-care diagnostics.

\subsection{Processes involved in an LOC}

Fluid control (actuator) units and sensors with microfluidics are essential to realise an LOC. Depending on the concerned applications, the components may vary. However, in general, a typical LOC would include sample handling, sample and reagent mixing, separation and finally detection $[6,10]$. In the following section, we review these steps.

\subsubsection{Sample handling}

\subsubsection{Sample injector}

Sample injection is the first step involved in an LOC, where the sample to be analysed is introduced from the external world into the chip using either syringe pumps or automated pipetting systems. Apart from an external world, the fluid has to be precisely transferred between different components of the LOC, such as to move fluids to the sensing sites where the target immobilise and bind to the surface bound sites. A class of devices called micropumps were developed by researchers initially with the goal of delivering therapeutic agents into a human body, such as an insulin delivery system [11]. However, with miniaturisation of the chemical and biological analysis, different types of micropumps have been realised for LOC devices. They are classified by fluid flow and pressure generated into either a displacement or dynamic micropumps [12]. A displacement micropump works by applying the pressure created by a moving boundary to drive the fluid. Some examples of the displacement micropumps includes piezoelectric [13], pneumatic [14], rotary [15], electromagnetic [16], electrostatic [17] and recently expanding field of electrowetting [18]. The use of complicated structures and requirement of large areas for the controlling the elastic elements in 
a displacement micropump renders them unsuitable for miniaturisation [19]. On the other hand, constant energy is imparted to the fluid to increase its momentum in a dynamic pump. Dynamic micropumps are classified by the driving field involved into acoustics (Flexural waves, the bubble generated streaming and SAW), electrokinetics (including electro-osmosis, electrophoresis, dielectrophoresis), optics (tweezers) and magnetohydrodynamics [20].

\subsubsection{Sample pretreatment}

Depending on the application, this step becomes essential to isolate or treat the required analyte from the complex sample. This phase becomes mandatory in certain molecular diagnostics involving DNA/RNA, as the nucleic acid is extracted from the whole cell. The extraction process improves the efficiency of the polymerase chain reaction $(\mathrm{PCR})$. The challenge involved in integrating the sample preparation step in microfluidics apart from handling the different formats of raw samples is the large variation in the preparatory step for various type of analysis. For example, extraction of DNA samples from whole blood requires a dilution, lysis and pre-concentration [21], separating plasma from red blood cells requires only pre-concentration [22] step while separating white blood cells from whole blood needs a filtration stage [23]. Hence, it becomes difficult to generate a general framework for the separation process. Despite the lack of a universal frame for the sample preparation step, sample preparation is done on-chip which reduces the time (in few mins) [21] and manual intervention compared to the conventional culture methods or molecular based methods which consume few hours to days for the process [24]. The process of separation is also performed in subsequent steps, after mixing of reagent and sample and hence will be discussed in detail in this chapter. 


\subsubsection{Microfluidic mixing}

Micromixer is a major component in the LOC system. The necessity comes from the slow reaction time caused by the low Reynolds number flow or the laminar flow. The only source of mixing is via diffusion, which might take a long time not acceptable for many applications and most importantly for an LOC device. Hence, there have to be some changes in the channel design or inclusion of an external agitation to realise fast mixing of liquids. In general, micromixers are classified into either passive or active mixers $[25,26]$.

Passive Micromixers do not rely on external energy sources to cause mixing of fluid. Instead, diffusion or chaotic advection gives rise to mixing. The principle behind the fluid mixing involves modification in the channel geometry (shape) to split, stretch, fold and break the flow [27]. Since no more external actuation sources are required, the passive structures are simple, robust, stable and very easy for integration. The passive micromixers suffer from the disadvantage of using relatively longer mixing periods to achieve the same mixing efficiency as active mixers [28].

Active micromixers use external perturbations to actuate the fluids. The active mixers are classified based on the energy source that drives the mixing. As shown in Table 2.1 we summarise the various active mixing schemes for microfluidics.

Various mixers have been reported so far, however, mainly studied case by case. As many factors influence the mixing performance including the dimension, fluid diffusivity, inertia (Peclet number) and mixing quantification methods. Only when these mixers are compared under same or at least comparable conditions, we can make accurate conclusions. However, in general, the active mixing could be more efficient as it involves moving parts. However, the active mixers requires actuators and corresponding controllers, which could make the system more complicated and increase fabrication cost. In comparison, for passive mixing, the fluids must flow through 
a certain length of the channel to get good mixing, and this may cause wastage of the precious liquid samples. The absence of moving parts and external controllers makes it simpler, cheaper and more reliable. From the operation point of view, passive mixing may be more suitable for continuous inline mixing; while active mixing can be used for both inline mixing and bath mixing (or mixing in static chambers).

\begin{tabular}{|c|c|c|c|c|}
\hline Mechanism & Principle & $\begin{array}{l}\text { Mixing } \\
\text { time }(\mathrm{ms})\end{array}$ & $\begin{array}{l}\text { Mixing- } \\
\text { index }\end{array}$ & Reference \\
\hline \multirow[t]{2}{*}{$\begin{array}{l}\text { Acoustic/ } \\
\text { Ultrasonic }\end{array}$} & $\begin{array}{l}\text { Using acoustics to drive trapped } \\
\text { microbubbles causing cavitation } \\
\text { microstreaming }\end{array}$ & 120 & 0.025 & [29] \\
\hline & $\begin{array}{l}\text { Surface acoustic wave induced } \\
\text { acoustic streaming }\end{array}$ & 600 & 0.9 & [30] \\
\hline $\begin{array}{l}\text { Electro hy- } \\
\text { drodynamic }\end{array}$ & $\begin{array}{l}\text { Motion of charge carriers in the } \\
\text { presence of an electrical field }\end{array}$ & 100 & $0.2-0.5$ & [31-33] \\
\hline $\begin{array}{l}\text { Dielectro } \\
\text { phoretic }\end{array}$ & $\begin{array}{l}\text { Force is exerted on a dielec- } \\
\text { tric particle subjected to a non- } \\
\text { uniform electric field, gives rise } \\
\text { to a chaotic trajectory and accel- } \\
\text { erates mixing }\end{array}$ & & 0.85 & {$[34,35]$} \\
\hline $\begin{array}{l}\text { Magneto hy- } \\
\text { drodynamic }\end{array}$ & $\begin{array}{l}\text { A mutually perpendicular elec- } \\
\text { tric field and magnetic fields act- } \\
\text { ing on a fluid generate Lorentz } \\
\text { force to drive mixing. }\end{array}$ & 1100 & 0.977 & [36] \\
\hline $\begin{array}{l}\text { Optics (laser } \\
\text { induced mix- } \\
\text { ing) }\end{array}$ & $\begin{array}{l}\text { Laser-induced formation of cav- } \\
\text { itation bubbles, which expands } \\
\text { and collapse to generate mixing. }\end{array}$ & 50 & $\begin{array}{l}\text { Capable of } \\
\text { starting an } \\
\text { enzyme- } \\
\text { catalyzed } \\
\text { reaction }\end{array}$ & [37] \\
\hline
\end{tabular}

Table 2.1: Various types of active micromixers

Among the various active mixing methods, acoustic-based mixers have reaped high importance due to their non-invasive nature, simple and inexpensive fabrication process and instrumentations [29,38-40]. 


\subsubsection{Separation}

This stage becomes significant in two classes of applications: one for purifying a sample from the other contaminants or unwanted objects, and the other involving applications where the segregated particle needs to be analysed. This part finds widespread applications in the field of biological analysis, chemical synthesis and mineral processing [41-44,44]. The most widely discussed application involves the separation of rare circulating tumour cells (CTCs) from blood for diagnostics [45, 46]. In the field of therapeutics, there is a significant interest towards using the separation methods to purify the blood (separating bacteria, CTCs, etc.) before transfusing to a donor [47]. In the realm of cell biology, it becomes necessary to separate cells of a particular type and study their behaviour [48]. Similar to the micromixing methods mentioned in the previous section, the separation technique is classified into fundamentally two approaches: active and passive. Additionally, there are a few variants, which combine the passive and active methods to achieve improved performance. Various review papers have discussed the different types of passive and active separation devices. The review paper by Wyatt et al. [49] and Lee et al. [50] discusses in detail various cell separation techniques. The thesis focuses on using a class of separation devices, which falls into the active separation category. The review papers from Gossett et al. [51], Sajeesh et al. [41] discusses elaborately on the different types of the passive separation device. A few of them includes hydrodynamic filtration based on size and shape, microscale filters depending on the size and deformability, another sorting method based on the dimension of the particle relative to the microchannel dimension involves the phenomenon involving the interplay of inertial and Dean forces. The passive device, in general, have a low throughput (less than $10 \mu \mathrm{l} / \mathrm{min}$ ), lower separation resolution ( $>5 \mu \mathrm{m}$ inertial) and lower purity or efficiency of segregation ( 90 $\%)$. On the contrary, an active method of separation ensures a high separation efficiency (100\%) [52], higher resolution of isolation $(<0.4 \mu \mathrm{m})$ [53] and high throughput 
(400 $\mu / / \mathrm{min})$ [54]. Table 2.2 shows a comparison of different active separation techniques for blood cells.

Among the different separation methods relying on their physical properties (label-free), the acoustic methods provide unique features. The acoustic separation of cells has little impact on its viability. It is an entirely label-free approach with no modifications needed on the medium in which the cells are cultured and separated. The Table 2.2 shows that acoustic-based method is the only active separation process that can differentiate cells based on size, density and compressibility, it becomes necessary to separate leukaemia cells from normal leukocytes with similar size but varying compressibility. Furthermore, the SAW devices used for separation are very compact (few square. centimetres) and uses lower power $(<1 \mathrm{~W})$, which makes it favourable for an LOC.

\subsubsection{Detection}

In the following section, we review the various detection techniques utilised in the microfluidic devices. The subsequent discussion builds on the review papers from [61-65]. The detection techniques mainly include optical, electrochemical, magnetic, mass spectrometry and biosensors. At the end of this section, a new sensing modality namely photoacoustics (PA), extensively used in imaging applications is introduced with capabilities for multi-parameter detection- optical and mechanical properties of the analyte. Table 2.3 lists the various detection approaches with an emphasis on the sensing in a microfluidic channel. 


\begin{tabular}{|c|c|c|c|c|c|c|c|}
\hline Method & Principle & $\begin{array}{l}\text { Separation } \\
\text { criteria }\end{array}$ & Sample & $\begin{array}{l}\text { Separation } \\
\text { resolu- } \\
\text { tion }(\mu \mathbf{m})\end{array}$ & $\begin{array}{l}\text { Through- } \\
\text { put } \\
(\mu \mathrm{L} / \mathrm{min})\end{array}$ & $\begin{array}{l}\text { Purity/ } \\
\text { effi- } \\
\text { ciency }\end{array}$ & $\begin{array}{l}\text { Refer- } \\
\text { ence }\end{array}$ \\
\hline Acoustic & $\begin{array}{l}\text { Acoustic radia- } \\
\text { tion force due } \\
\text { to a travelling or } \\
\text { standing acous- } \\
\text { tic wave using } \\
\text { SAW or ultra- } \\
\text { sound transduc- } \\
\text { ers }\end{array}$ & $\begin{array}{l}\text { Size, density } \\
\text { and com- } \\
\text { pressibility }\end{array}$ & $\begin{array}{l}\text { Cell } \\
\text { (BAW) } \\
\text { MCF-7 } \\
\text { from WBC } \\
\text { Polystyrene } \\
\text { particles }\end{array}$ & $\begin{array}{l}>1 \\
0.2 \\
0.2-0.4\end{array}$ & $\begin{array}{l}80 \\
2 \\
4-6\end{array}$ & $\begin{array}{l}100 \% \\
80 \% \\
80-90 \%\end{array}$ & $\begin{array}{l}{[55]} \\
{[52]} \\
{[53,56]}\end{array}$ \\
\hline Magnetic & $\begin{array}{l}\text { Magnetophore- } \\
\text { sis enabled } \\
\text { by permanent } \\
\text { magnets or } \\
\text { coils exert- } \\
\text { ing force on } \\
\text { cells labelled } \\
\text { with magnetic } \\
\text { particles } \\
\text { Magnetic force } \\
\text { on particles due } \\
\text { to difference } \\
\text { in magnetic } \\
\text { susceptibility } \\
\text { to the bulk and } \\
\text { magnetic field } \\
\text { gradient }\end{array}$ & $\begin{array}{l}\text { Size } \\
\text { Using native } \\
\text { magnetic } \\
\text { susceptibility }\end{array}$ & $\begin{array}{l}\text { CTCs } \\
\text { from } \\
\text { mouse } \\
\text { blood } \\
\\
\\
\text { isolating } \\
\text { rare nu- } \\
\text { cleated } \\
\text { red blood } \\
\text { cells from } \\
\text { peripheral } \\
\text { blood }\end{array}$ & $\begin{array}{l}\text { Not based } \\
\text { on size but } \\
\text { separate } \\
\text { paramag- } \\
\text { netic from } \\
\text { diamag- } \\
\text { netic }\end{array}$ & $216-450$ & $90 \%$ & [54] \\
\hline $\begin{array}{l}\text { Dielec- } \\
\text { trophore- } \\
\text { sis (DEP) }\end{array}$ & $\begin{array}{l}\text { Force exerting } \\
\text { on a polarizable } \\
\text { particle due to } \\
\text { a non-uniform } \\
\text { electric field. } \\
\text { Particles need } \\
\text { not be intrinsi- } \\
\text { cally charged }\end{array}$ & $\begin{array}{l}\text { Polarizability, } \\
\text { size }\end{array}$ & $\begin{array}{l}\text { Lipoo- } \\
\text { somes }\end{array}$ & $\begin{array}{l}\quad 0.5 \mu \mathrm{m} \\
\text { (particle } \\
\text { size > }>1 \\
\mu \mathrm{m} \text {, due } \\
\text { to lack of } \\
\text { effective } \\
\text { focussing) }\end{array}$ & 6 & $90 \%$ & [58] \\
\hline Optical & $\begin{array}{l}\text { Formation of an } \\
\text { optical lattice or } \\
\text { arrays through } \\
\text { multiple beam } \\
\text { interference } \\
\text { CCD-enabled } \\
\text { detection fol- } \\
\text { lowed by cell } \\
\text { transporta- } \\
\text { tion by optical } \\
\text { tweezers }\end{array}$ & $\begin{array}{l}\text { Refractive in- } \\
\text { dex, size } \\
\text { Size and fluo- } \\
\text { rescence }\end{array}$ & $\begin{array}{l}\text { Particles } \\
\\
\text { yeast } \\
\text { cells and } \\
\text { human } \\
\text { embryonic } \\
\text { stem cells }\end{array}$ & $<2$ & $\begin{array}{l}1500 \text { par- } \\
\text { ticles/min } \\
10 \mathrm{~nL} / \mathrm{min}\end{array}$ & $96 \%$ & [60] \\
\hline
\end{tabular}

Table 2.2: Comparison of active separation techniques found in existing lab-on-a-chip systems 


\begin{tabular}{|c|c|c|c|c|c|}
\hline Method & Principle & Sensitivity & Advantage & Disadvantage & Ref \\
\hline Fluorescence & $\begin{array}{l}\text { The phenomenon } \\
\text { of light emission by } \\
\text { a molecule (fluo- } \\
\text { rophore) after initial } \\
\text { electronic excita- } \\
\text { tion due to light } \\
\text { absorption. The } \\
\text { emitted light has a } \\
\text { wavelength higher } \\
\text { than the excitation } \\
\text { wavelength }\end{array}$ & $\begin{array}{l}\text { Detected } 10-100 \\
\mathrm{nM} \text { of Rhodamine } \\
6 \mathrm{G} \\
\text { Detected } 10 \mathrm{nM} \\
\text { of Alexa Fluor } 633 \\
\text { dye }\end{array}$ & $\begin{array}{l}\text { Low detection limit } \\
\text { High selectivity }\end{array}$ & $\begin{array}{l}\text { Requires label (sample } \\
\text { tagging)-Time consuming } \\
\text { as it requires fluid handling, } \\
\text { mixing and washing steps } \\
\text { Fluorescent dyes are } \\
\text { costly, limited shelf life and } \\
\text { affected by pH which varies } \\
\text { between samples } \\
\text { Isolate leakage excitation } \\
\text { light from the detector, calls } \\
\text { for additional polarisation } \\
\text { schemes to block }\end{array}$ & [66-69] \\
\hline $\begin{array}{l}\text { Chemilumines- } \\
\text { cence }\end{array}$ & $\begin{array}{l}\text { A chemical reaction } \\
\text { generates light }\end{array}$ & $\begin{array}{l}\text { Detect hydrogen } \\
\text { peroxide with LOD } \\
=5 \mu \mathrm{m}\end{array}$ & $\begin{array}{l}\text { Excitation light } \\
\text { source and emis- } \\
\text { sion filters are } \\
\text { not required- Re- } \\
\text { duced background } \\
\text { interference }\end{array}$ & $\begin{array}{l}\text { Require highly sensitive } \\
\text { detectors } \\
\text { Require chemi-luminescent } \\
\text { labels for detection }\end{array}$ & [70] \\
\hline $\begin{array}{l}\text { UV-Vis ab- } \\
\text { sorbance }\end{array}$ & $\begin{array}{l}\text { Relies on the opti- } \\
\text { cal absorption or at- } \\
\text { tenuation at a par- } \\
\text { ticular optical wave- } \\
\text { length }\end{array}$ & $\begin{array}{l}\text { Alexa Fluor } 633 \text { dye } \\
\text { detected at } 1 \mu \mathrm{M} \\
\text { BSA detected } \\
\text { at } 15 \mathrm{nM} \\
\text { Coumarin dye } \\
\text { with an LOD = } 4.3 \\
\mu \mathrm{M}\end{array}$ & $\begin{array}{l}\text { Label-free approach } \\
\text { Instrumentation } \\
\text { is simple }\end{array}$ & $\begin{array}{l}\text { Decreased sensitivity } \\
\text { due to decreased optical } \\
\text { path length and hence } \\
\text { reduced absorbance (by } \\
\text { Beer-lambert law) }\end{array}$ & {$[68,71,72]$} \\
\hline $\begin{array}{l}\text { Electrochemical } \\
\text { detection }\end{array}$ & $\begin{array}{l}\text { Cyclic voltammetry } \\
\text { is an amperometric } \\
\text { technique. The volt- } \\
\text { age is varied, and } \\
\text { the current induced } \\
\text { is detected., The } \\
\text { limiting current is } \\
\text { proportional to an- } \\
\text { alyte concentration } \\
\text { by the Nernst equa- } \\
\text { tion }\end{array}$ & $\begin{array}{l}\text { RNA detection in } \\
\text { saliva }=0.4 \mathrm{fM}\end{array}$ & $\begin{array}{l}\text { Wide dynamic } \\
\text { range } \\
\text { Low cost }\end{array}$ & $\begin{array}{l}\text { Electrodes may get fouled } \\
\text { due to by-products of the } \\
\text { reactions } \\
\text { Lower response time - } \\
\text { time for the analyte to } \\
\text { diffuse to the electrode } \\
\text { surface }\end{array}$ & [73] \\
\hline
\end{tabular}




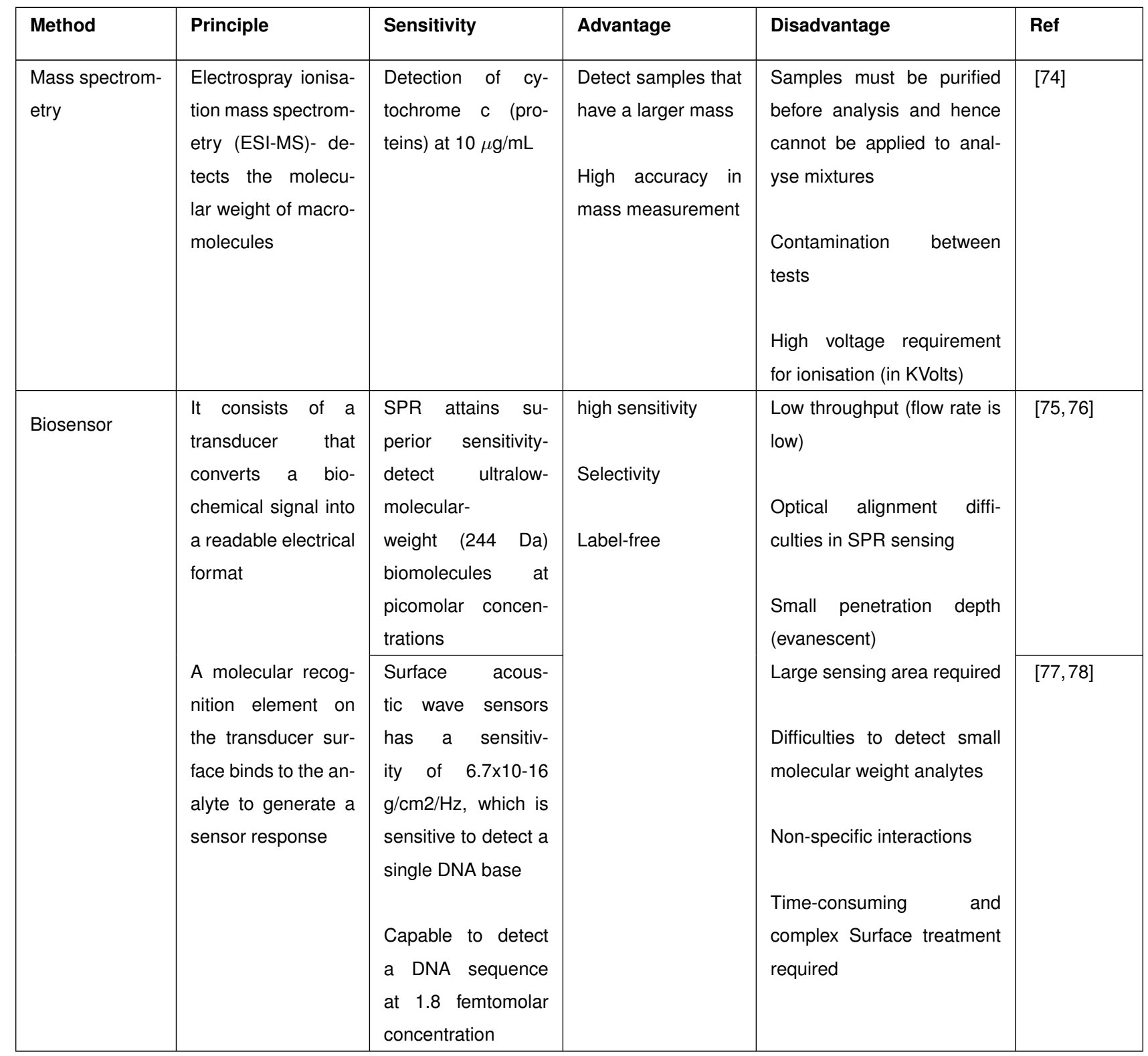

Table 2.3: Comparison of the detection techniques found in existing lab-on-a-chip systems

Though biosensors (including SPR and SAW) possess high sensitivity, they lack the throughput for detecting rare analytes (e.g., circulating tumour cells such 1100 per $1 \mathrm{~mL}$ of blood) and require tedious pre-treatments before detection. In this context, volumetric or bulk detection possess advantages. The conventional opti- 
cal sensors (in Table 2.3), photonic crystal liquid sensors [79] and optomechanofluidics [80] operate directly on the bulk fluid. Recently there is much emphasis on a technique utilising laser-generated acoustic wave in both sensing and imaging applications. Photoacoustic (PA) is the physics of generating an acoustic wave due to absorption of a pulsed laser irradiation on a sample [81]. In the arena of biomedical imaging, the PA technique achieves much significance as it combines the advantages of strong optical contrast and high-resolution acoustic detection (reduced scattering compared to the light). The PA is also used for trace gas detection (in ppb levels) $[82,83]$. The capability of spectroscopy with sensitive detection is highly beneficial for multi-analyte detection. PA has been applied recently for single cell detection. Also, the PA flow cytometry was used for malaria diagnosis with detection sensitivity thousand times better than existing tests [84]. Since the PA fundamentally works on the optical absorption principle, as mentioned in Table 2.3, it is a label-free method. In comparison to other optical detection techniques, PA is ideally, a background-free technique, since the signal is generated only by the absorbing analyte. On the other hand, conventional absorption detection is an indirect method, as it uses the reflected or transmitted signals. The transmitted signal is severely affected by low absorption coefficients, opaque samples and scatterers inside the sample. PA overcomes these limitations to form an optimum detector [85]. Similar to the fluorescence detection, the PA signal depends on the incident power. The PA signal is linearly proportional to the laser fluence (energy per unit area) and absorption coefficient of the sample [81, 86, 87]. Fig 2.1 shows the principle of PA signal generation, where the sample analyte absorbs laser excitation (nanosecond pulse width). The electromagnetic wave absorption generates heating in the sample. A ultrashort pulse duration prevents heat loss during the laser irradiation period. Thermal and stress confinement are satisfied by the nanosecond laser pulse. The thermal expansion converts into a longitudinal acoustic wave, detected using an ultrasound transducer. 


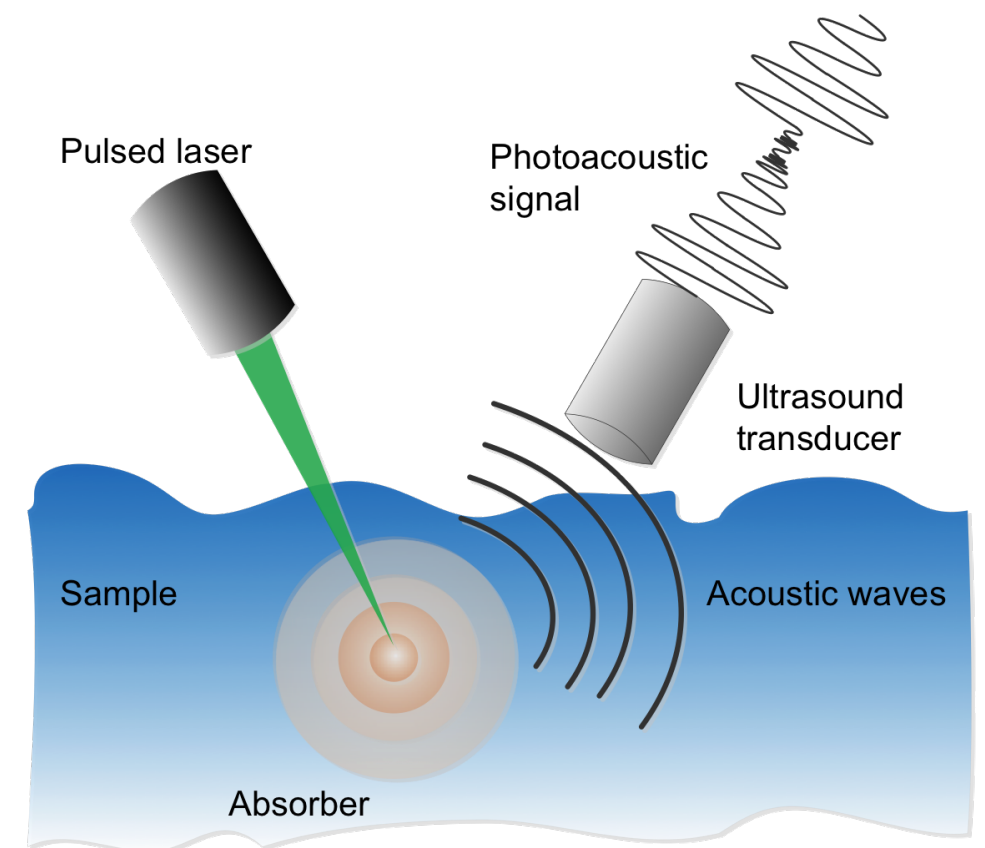

Figure 2.1: Photoacoustic principle- generation of Photoacoustic (PA) signal due to a pulsed laser excitation [88].

\subsection{Surface acoustic wave microfluidics}

Surface acoustic wave (SAW) are nanomechanical waves $(\sim \mathrm{nm}$ to $\AA$ ) propagating on a piezoelectric material [89-91]. The elastic wave that propagates on the surface of the piezoelectric material (like lithium niobate, quartz, etc.) is also known as Rayleigh waves. Unlike in bulk, at the surface, the longitudinal and transverse modes are coupled. Due to different displacements of the modes, the Rayleigh waves are elliptically polarised. Another important property of the surface waves is the evanescent behaviour of the waves and hence the particle displacements decay inside the substrate within one-two wavelengths. See Fig 2.2(a) for the acoustic displacement profile along the piezoelectric substrate. SAW is generated in a piezoelectric material through various methods including interdigital transducers (IDT), non-contact excitation user pulsed laser [92] and wedge-coupled ultrasound transducer [93]. Fig 2.2(b) shows the most commonly used method of SAW excitation using an inter-digital trans- 
ducer (IDT) patterned the piezoelectric substrate. The IDT also acts as a narrow band receiver of the surface acoustic wave. The design specification of a SAW device involves finding the interdigital electrode (IDT) width for a certain frequency. As shown in Fig 2.2(c), The inter-finger spacing $d$ is the design parameter for a specific frequency

$$
d=v / 4 f
$$

Here, $v$ is the velocity of the surface acoustic wave and $f$ is the frequency. However, the above design requires the exact value of the SAW velocity, which might change due to various second order effects such as bulk acoustic wave radiation, inter-finger reflection, etc. One of the standard design techniques to reduce the unwanted finger reflections is to make the use of a split-electrode $(\lambda / 8)$ design. Here, $\lambda$ refers to the wavelength of the SAW. Hence, the IDT width $d$ will b $\lambda / 8$ instead of $\lambda / 4$ [94]. SAW has been widely used for communication devices for the design of RF and IF filters for mobile phones, high- $Q$ resonators and receivers for precision filtering. The ability to perform complex signal processing within a small footprint and mass-volume fabrication capabilities are prime factors contributing to the widespread use of SAW devices in communication applications $[95,96]$. Over the past decade, the surface acoustic wave has been receiving significant attention in the field of microfluidics [97-99]. Compared to conventional ultrasound or bulk acoustic waves (BAW), the concentration of SAW energy within the surface of the piezoelectric substrate causes higher efficient transfer of acoustic energy into the fluid at low power $(<1 \mathrm{~W})$. Also, the relatively (BAW and ultrasound) high frequency ( $\mathrm{MHz}$ order) SAW is capable of generating high accelerations $\left(108 \mathrm{~m} / \mathrm{s}^{2}\right)$ to drive a multitude of microfluidic applications. A difference in the acoustic wave velocity in the piezoelectric substrate $\left(4000 \mathrm{~m} / \mathrm{s}^{2}\right.$ for $128^{\circ} \mathrm{YX}$ lithium niobate, $\mathrm{LiNbO}_{3}$ ) and fluid $\left(1500 \mathrm{~m} / \mathrm{s}^{2}\right.$ ) loaded on the substrate causes wave to refract into the liquid. The longitudinal wave inside the fluid causes flow via a phenomenon known as acoustic streaming [99]. Yeo et al. [98] explain the existence of different forms of acoustic streaming. Based on the various length scale of the system the 


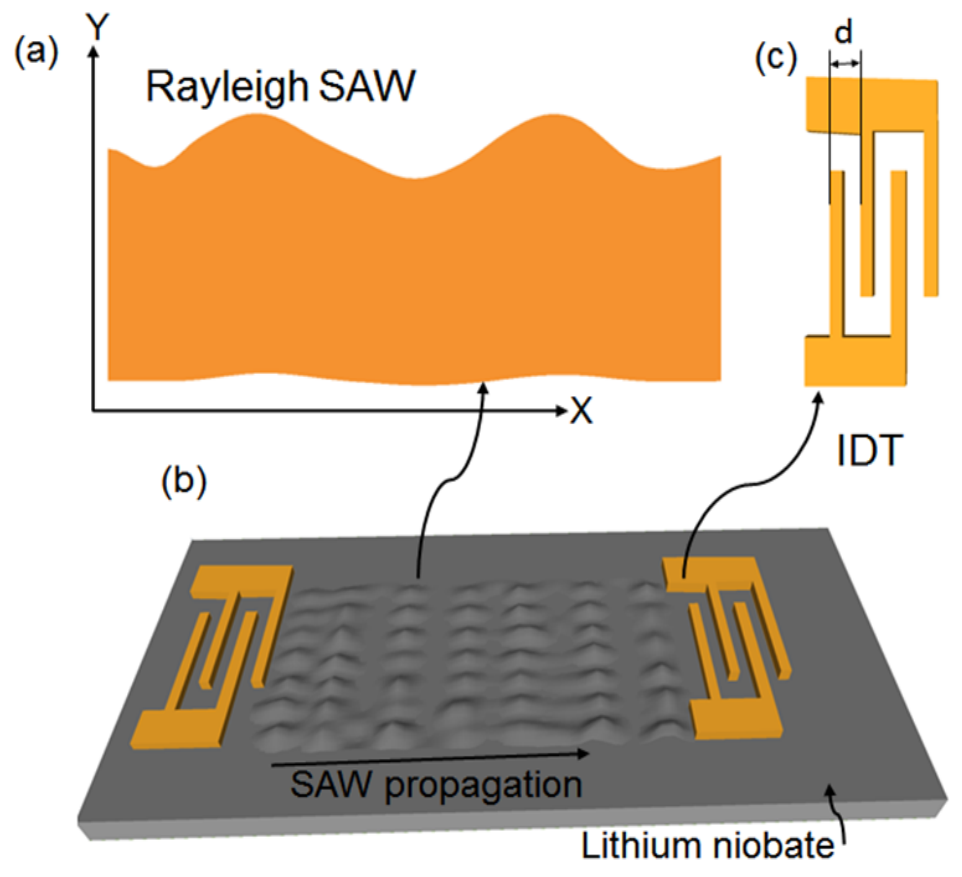

Figure 2.2: Schematic of a surface acoustic wave device. (a) Displacement profile for a Rayleigh SAW propagation (elliptical motion on the surface). Displacement decays within two wavelength. (b) A SAW delay line (two IDTs) with the wave propagation along the piezoelectric substrate (lithium niobate, $\mathrm{LiNbO}_{3}$ ). (c) General configuration of the IDT to excite SAW. $d$ denotes the electrode pitch.

acoustic streaming can be classified as Schilting streaming, Rayleigh streaming and Eckart streaming. Fig 2.3 shows a SAW wave generated from an IDT interacting with a droplet on the substrate. Fig 2.3(b) shows the wave refracting into the droplet following the Snells law of refraction. A Rayleigh wave on interacting with a droplet leaks energy into the fluid and hence a leaky SAW is generated on the substrate. For particle manipulation using SAW, either a standing SAW (SSAW) or travelling SAW (TSAW) are employed [53]. The use of SSAW to actuate particles is termed as acoustophoresis. In SSAW, two oppositely directed travelling SAW (from two IDTs) interfere to create a standing acoustic wave. The SSAW is manipulated to either trap [100] and move $[101,102]$ cells/particles at either the pressure nodes/antinodes of the SSAW. Four forces are acting on a particle: acoustic radiation force, viscous force (drag force), buoyant force and gravity. The last two of the forces are typically neglected, as the two 
(a)

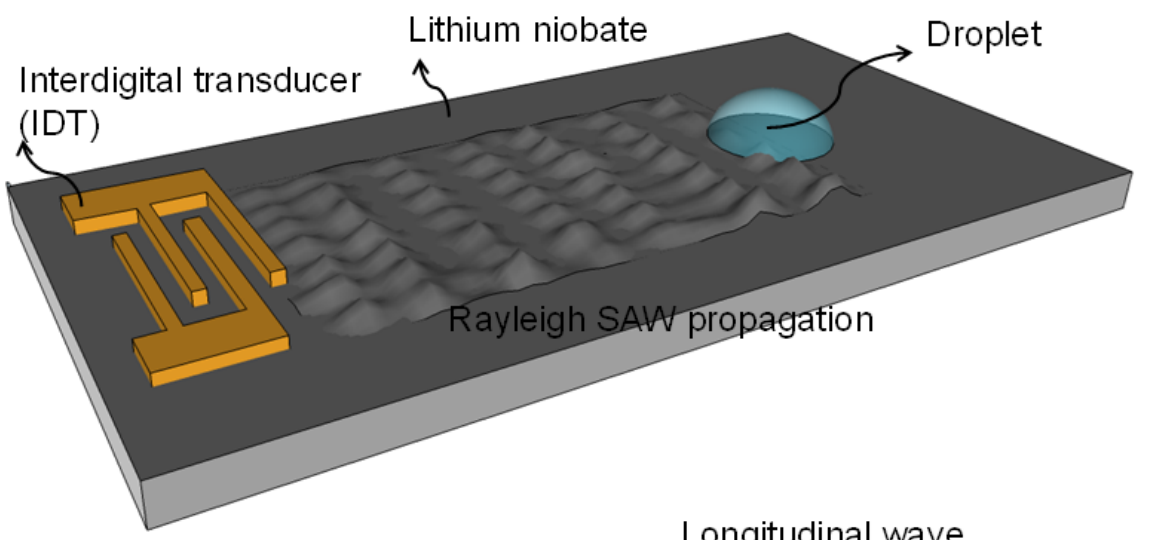

(b)

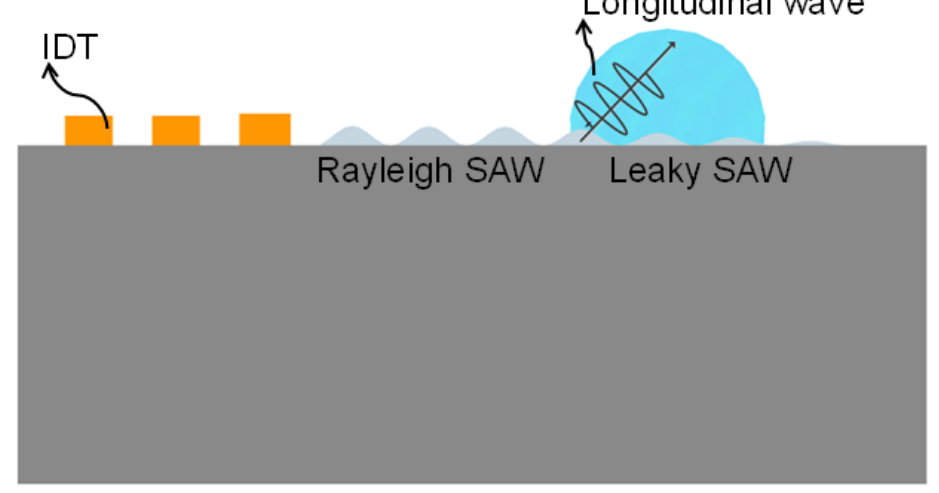

Figure 2.3: (a) Schematic of the SAW (not to scale) interacting with a droplet. (b) A cross-sectional view of a SAW refracting into the fluid and inducing acoustic streaming. The direction of the propagation of longitudinal wave inside the fluid is determined by the ratio of the velocity of sound in the piezoelectric substrate and fluid by the Snells law.

forces are equal in magnitude and opposite in direction. The acoustic radiation force (Fr) causes the particles to move to either the nodes/antinodes [103].

$$
f_{r}=4 \pi \phi\left(\beta^{\prime}, \rho^{\prime}\right) k a^{3} E_{\mathrm{s}} \sin (2 k x)
$$

where $\phi$ is the acoustic contrast factor depending on the compressibility $\left(\beta^{\prime}\right)$ and density $\left(\rho^{\prime}\right)$ of the fluid and particle, $k$ the wave-number, a the radius of the particle, $E_{s}$ the acoustic energy density of the standing wave and $x$ is the distance from a pressure node along the acoustic wave propagation direction. The particle movement under the 
acoustic field creates a viscous drag force $\left(F_{v}\right)$ to oppose the motion, given by:

$$
f_{v}=-6 \pi \eta a v_{\mathrm{p}}
$$

where $\eta, v_{p}$ denotes the medium viscosity and particle velocity respectively. The force $F_{v}$, together with the acoustic force $F_{r}$ determines how fast the particles travel to the nodes/antinodes. The acoustophoretic contrast factor $(\phi)$ in the radiation force equation determines if the particles aggregate at nodes $(\phi>0)$ or the antinodes $(\phi<0)$.

$$
\phi\left(\beta^{\prime}, \rho^{\prime}\right)=\frac{1}{3}\left[\frac{5 \rho^{\prime}-2}{2 \rho^{\prime}+1}-\beta^{\prime}\right]
$$

$\rho^{\prime}=\frac{\rho \mathrm{p}}{\rho_{0}}$ and $\beta^{\prime}=\frac{\beta_{\mathrm{p}}}{\beta_{0}}$ are the relative density and compressibility respectively for the particle (subscript, p) and fluid (subscript,0) respectively. The acoustic radiation force generated by a plane progressive (TSAW) wave on a particle is given by [104]:

$$
f_{r}=4 \pi \phi\left(\beta^{\prime}, \rho^{\prime}\right) k^{4} a^{6} E_{\mathrm{p}}
$$

Rearranging Eq. 2.2 and Eq. 2.5 as explained by Groschl [104], the $f_{r} \propto(k a)^{3}$ and $f_{r} \propto(k a)^{6}$ for the SSAW and TSAW respectively. The Eq. 2.2 and Eq. 2.5 are derived with the condition that (particle size < wavelength). Hence, it can be concluded that an SSAW generates larger acoustic radiation force than a TSAW.

\subsubsection{SAW fluid actuation and manipulation}

The SAW generated acoustic streaming, and acoustic radiation force is utilised for various applications in microfluidics to manipulate both fluid and particles. The TSAW is applied in both open and closed microfluidic channel to achieve fluid mixing, pumping (translation), jetting, particle concentration, and controlling nanoparticles. The standing wave formed by SSAW is used in applications including focussing 
particles, separation based on acoustic properties (density, size and compressibility), patterning particles in a stagnant fluid using $2 \mathrm{D}$ and $3 \mathrm{D}$ tweezers and to align nanomaterials. The review paper by Ding et al. [97] and Friend et al. [99] provides an exhaustive review of the various applications realised using SAW microfluidics. SAW techniques are suitable for such applications mainly because of the unique features of being compact, inexpensive [105], compatible with bio-relevant samples [101,106], energy efficient (energy is concentrated only on the surface) and versatile in handling different fluids regardless of their electrical, magnetic or optical properties. Moreover, SAW provides contact-free manipulation of liquids. SAW is also coupled to disposable superstrate avoids contamination of the substrates and makes it reusable for multiple analysis.

\begin{tabular}{|c|c|c|c|c|}
\hline & $\begin{array}{l}\text { Electrohydro- } \\
\text { dynamic }\end{array}$ & $\begin{array}{l}\text { Dielec- } \\
\text { trophoretic }\end{array}$ & $\begin{array}{l}\text { Magnetohy- } \\
\text { drodynamic }\end{array}$ & $\begin{array}{l}\text { Acous- } \\
\text { tic(SAW) }\end{array}$ \\
\hline Working fluid & Dielectric fluids & $\begin{array}{l}\text { Wide range de- } \\
\text { pends on ionic } \\
\text { density and } \mathrm{pH}\end{array}$ & $\begin{array}{l}\text { Only on elec- } \\
\text { trolyte solution }\end{array}$ & Any fluids \\
\hline $\begin{array}{l}\text { Approximate } \\
\text { size }\left(\mathrm{mm}^{3}\right)\end{array}$ & $10-250$ & $100-1000$ & $\begin{array}{l}\text { Depends on re- } \\
\text { quired magnetic } \\
\text { field }\end{array}$ & $5-10$ \\
\hline $\begin{array}{l}\text { Operating volt- } \\
\text { age }(\mathrm{V})\end{array}$ & $40-600$ & $40-1500$ & $\begin{array}{l}\text { Low voltage } \\
(\sim 1 \mathrm{~V}) \text { but also } \\
\text { require to gen- } \\
\text { erate magnetic } \\
\text { field }\end{array}$ & $30-40$ \\
\hline Reusable & No & No & No & Yes \\
\hline $\begin{array}{l}\text { Microfluidic ap- } \\
\text { plications }\end{array}$ & Continuous & Continuous & Continuous & $\begin{array}{l}\text { Both droplet } \\
\text { and continuous } \\
\text { microfluidics } \\
\text { are supported }\end{array}$ \\
\hline Effects on fluid & Joule heating & Joule heating & Joule heating & $\begin{array}{l}\text { Pulsed } \\
\text { excitation- } \\
\text { reduces fluid } \\
\text { heating }\end{array}$ \\
\hline
\end{tabular}

Table 2.4: Advantages of SAW microfluidics 
Table 2.4 shows the comparison of various active microfluidic actuation techniques. Hence we can conclude that the acoustic wave technology is a good solution for a lab-on-chip platform (smaller size, lower power, absence of physical contact with the liquid and ability to work with most of the fluids). 


\section{Chapter 3}

\section{Acoustofluidic mixing: The study of}

\section{an acoustically coupled multi-layered microfluidic platform on SAW}

\section{substrate}

As described in Chapter 1, one of the advantages about SAW microfluidics is its possibility to be reusable. However, existing literature shows that the coupling to superstrates involves losses with roughly 50\% transmission efficiency [107]. Hence, it becomes essential to optimise the device design to reduce the dissipative losses through the coupling layer and superstrates. Optimisation of the reusable microfluidic platform on surface acoustic wave (SAW) requires a clear understanding of the various factors that affects the acoustic energy transmission to the fluid in the microchannel. This chapter reports the characterization and analysis of the reusable SAW microfluidic platform. The acoustic wave was coupled through a liquid layer to a disposable superstrate. Using the microfluidic mixing phenomenon we characterised the acoustic 
energy transfer through various layers. We conducted experiments and developed new analytical models to relate the influence of the input voltage, frequency and coupling layer thickness to the mixing efficiency. The anti-symmetric higher order Lamb waves generated on thin glass plate makes compressional waves in the liquid to induce fluid motion. The acoustic energy delivered to the fluid increased as the square of the applied voltage and saturated at $50 \mathrm{~V}$. The frequency response demonstrated a higher acoustic energy transmission for the $100 \mathrm{MHz}$ compared to the $50 \mathrm{MHz}$. By detailed numerical modelling, we show that the existence of second order Lamb wave mode (A2) in the $100 \mathrm{MHz}$ device relative to the first order mode (A1) existing in the $50 \mathrm{MHz}$ improves the transmission efficiency. Power transmitted through the coupling layer displayed a sinusoidal dependence on the normalised thickness of the layer. Finally, we measured the temperature rise and calculated the variance in sound speed, and this is analysed to confirm the validity of the developed models. The contents of this chapter is based on our work given in [108].

\subsection{Introduction}

A recent review discussed the growing interest in using the disposable superstrates on the expensive piezoelectric substrates [97]. SAW coupling to a glass superstrate using water as a couplant was reported previously [107]. Disposable glass superstrate was also used to demonstrate droplet actuation using slanted finger interdigital transducer (IDT) [109].

Rapid mixing on liquid droplets using SAW as the primary force was demonstrated earlier by different groups [110-112]. The quantitative study relating the efficiency of the chaotic mixing process due to SAW for variations in the fluid viscosity and input power was also performed in a microfluidic well [113]. The effect of SAW was used as a mixer in polydimethylsiloxane (PDMS) microchannel bonded to the lithium niobate $\left(\mathrm{LiNbO}_{3}\right)$ substrate [114].They had analysed the effect of the channel orienta- 
tion on mixing efficiency. At electrical signals of 35 volts and a frequency of $9.6 \mathrm{MHz}$, a mixing efficiency of $94 \%$ was obtained.Sritharan et al. [115] reported on using SAW for mixing at low Reynolds number flow. They made use of a SAW device at $146 \mathrm{MHz}$ and observed the mixing behaviour for water and beads through a $Y$ shaped channel. Tan et al. [116] introduced channels by etching the $\mathrm{LiNbO}_{3}$ substrate and studied the mixing behaviour for different frequencies. They observed that when the channel length is larger than the wavelength of the sound at a particular frequency, a uniform to mixing flow transition occurs. The two ends of the microchannel reflect the SAW wave and hence form a transverse standing wave across the channel width. Luong et al. [117] proposed the use of a focussed interdigital transducer (F-IDT) to improve mixing. The F-IDT gives a mixing efficiency of $90 \%$ compared to the $10 \%$ efficiency obtained with a parallel IDT for a voltage of $80 \mathrm{~V}$ with Peclet number of 74400 and flow rate of 167 $\mu \mathrm{L} /$ min. This improvement in efficiency is because the F-IDT focusses the wave to a small area. However, the temperature of the substrate increases at the focal point due to wave focusing. Johansson et al. [118] used polydimethylsiloxane (PDMS) channels as a superstrate for particle manipulations in continuous-flow microfluidic operation, and performed a detailed evaluation of the acoustic wave coupling mechanisms and distribution of acoustic nodes in the fluid relative to the channel walls for effective particle manipulations. A qualitative visualisation of the effects of PDMS microchannel in the acoustic waves within the fluid is performed using finite element methods (FEM). In our work, we use mixing phenomenon to characterise the acoustic energy transfer through the various layers.

The reusable platform consists of microchannel created on a polydimethylsiloxane (PDMS) bonded to a disposable superstrate (glass), and acoustically coupled to the piezoelectric substrate. The fluid motion in the microchannel is caused due to the higher order antisymmetric Lamb wave modes generated on the glass superstrate, which acts a thin plate [107]. The antisymmetric mode with prominent transverse vibrations is coupled to the fluid in the microchannel causing fluid motion. This chapter 
focuses to characterise SAW transmission in the liquid channel in a direct manner, which is through mixing. We established a novel mechanism for characterising the SAW energy transmission in fluidic channels, which is essential for all the SAW microfluidics design, using a mixing structure. The main contribution of the work comes from the discussion of the effect of thickness of coupling layer and frequency on the mixing efficiency in a Y-shaped microchannel and development of analytical models. The analytical models that are developed in this chapter can be used to optimise the power transmission coefficient and hence increase mixing efficiency. The platform can be extended to other SAW-based microfluidic applications. The decoupling of the expensive piezoelectric substrate with the microfluidic device enables lower cost and disposability. This makes the platform an ideal fit for the development of point of care clinical diagnostics [119], screening or analytical tests [120].

\subsection{Materials and methods}

\subsubsection{SAW device design and simulation}

The anisotropic piezoelectric substrate used was a $\mathrm{LiNbO}_{3}$ wafer with the crystal cut rotated $128^{\circ}$ around the Y-axis. A double-electrode transducer was used to reduce the inter-finger reflection and improve its performance [94]. Table 3.1 shows the design specifications of the IDT for the frequencies of $50 \mathrm{MHz}$ and $100 \mathrm{MHz}$.

\begin{tabular}{|c|c|c|}
\hline \multirow{2}{*}{ Parameter } & \multicolumn{2}{|c|}{ Value } \\
\cline { 2 - 3 } & $50 \mathrm{MHz}$ & $100 \mathrm{MHz}$ \\
\hline IDT pitch $(\lambda / 8)-\mu \mathrm{m}$ & 9.7 & 4.765 \\
\hline Number of IDT pairs & 50 & 50 \\
\hline IDT thickness- $\mu \mathrm{m}$ & 0.15 & 0.15 \\
\hline Aperture-mm & 4 & 3 \\
\hline
\end{tabular}

Table 3.1: SAW design parameters 


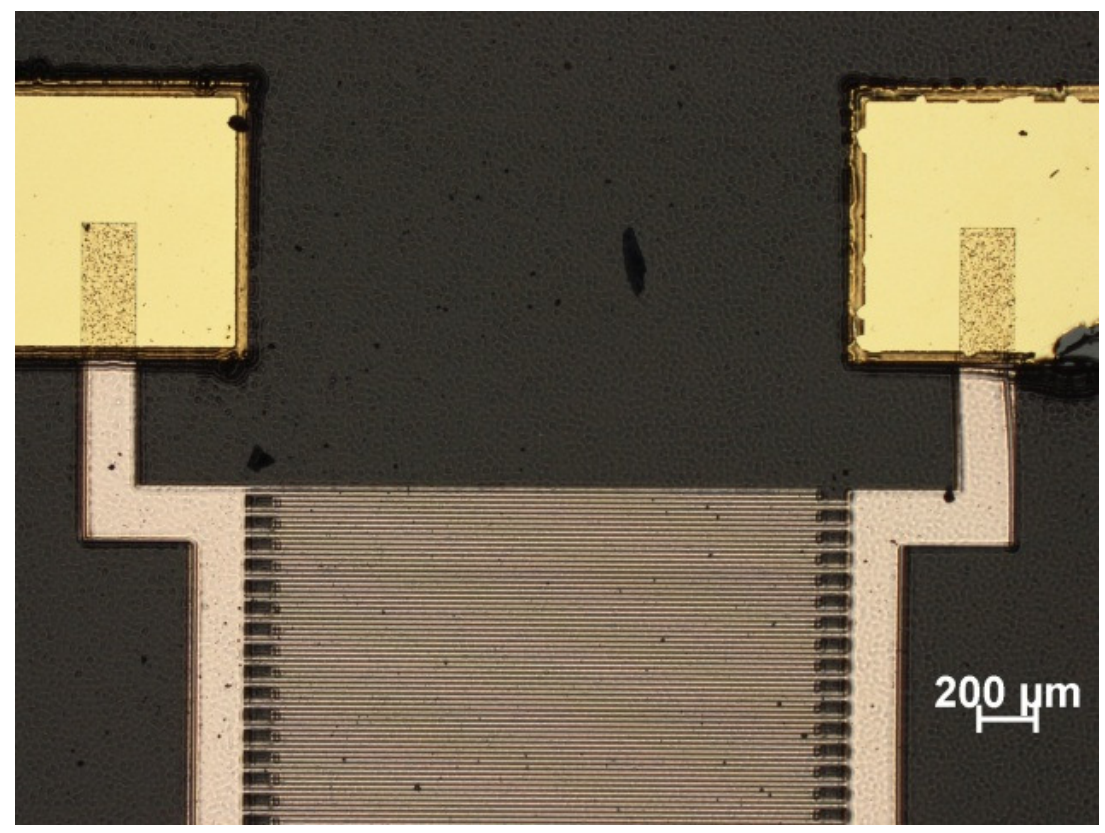

Figure 3.1: Fabricated SAW devices at frequency of $50 \mathrm{MHz}$. The yellow regions show the gold pads and the fine lines show the IDTs

\subsubsection{SAW device fabrication}

The fabrication process of the SAW device begins with a layer of aluminium patterned (thickness of $300 \mathrm{~nm}$ ) on the $128^{\circ}$ rotated $\mathrm{Y}$-cut $\mathrm{LiNbO}_{3}$ substrate using a positive photoresist to form the IDT. After patterning and stripping of the photoresist, the gold of thickness $1.5 \mu \mathrm{m}$ was then sputtered to create the metal pads for the contacts. To protect the IDT from oxidation, an additional layer of AZ-4620 photoresist was deposited by evaporation, for a thickness of $0.8 \mu \mathrm{m}$, except in the SAW propagation area. The final fabricated device is shown in Fig 3.1.

\subsubsection{SAW device characterization}

The fabricated device was bonded to a printed circuit board (pcb). The centre frequency of the fabricated device was characterized using the E5061B ENA Series Network Analyzer.Maximum acoustic power was generated from the SAW device at this frequency. The tolerance of the IDT width was $2 \%$ and $4 \%$ for the devices working 

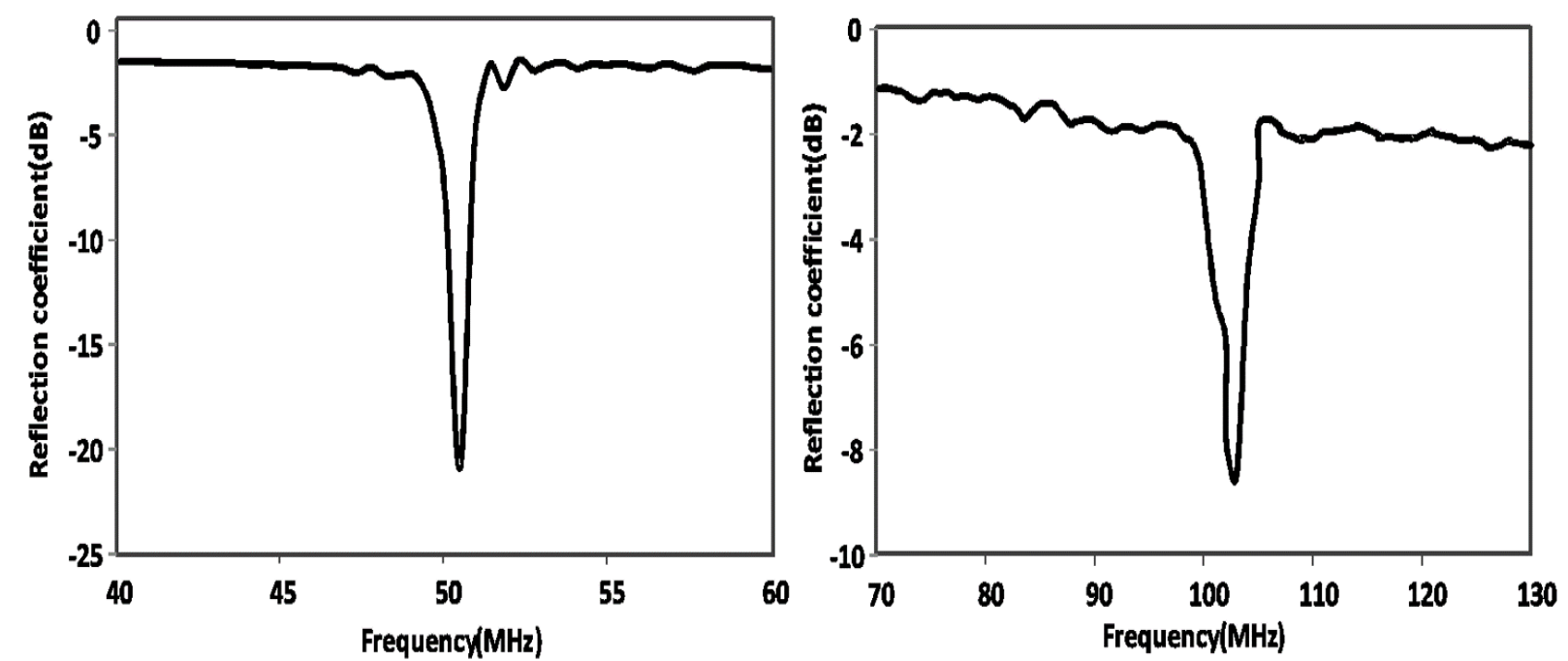

Figure 3.2: Measured S11 using the network analyser for the two devices at frequencies (a) $50.5 \mathrm{MHz}$ and (b) $103 \mathrm{MHz}$. The devices were designed for $50 \mathrm{MHz}$ and 100 $\mathrm{MHz}$ respectively.

at frequencies of $50 \mathrm{MHz}$ and $100 \mathrm{MHz}$ respectively. This could be induced by the photomask fabrication and photolithographic process, which in turn caused variance to the centre frequency. Fig 3.2 shows the $\mathrm{S} 11$ (reflection coefficient) for the fabricated devices, which was measured to be $-20 \mathrm{~dB}$ at $50 \mathrm{MHz}$ and $-8 \mathrm{~dB}$ at $100 \mathrm{MHz}$.

\subsubsection{Micromixer design}

Fig 3.3 shows the schematic of the reusable micromixing platform. The SAW was transmitted from the piezoelectric substrate through a coupling layer of water and a glass superstrate into the fluid flowing through the microchannel. A Y-shaped microchannel made of PDMS enclosed the liquid. A reservoir was specially designed to hold the water couplant. 


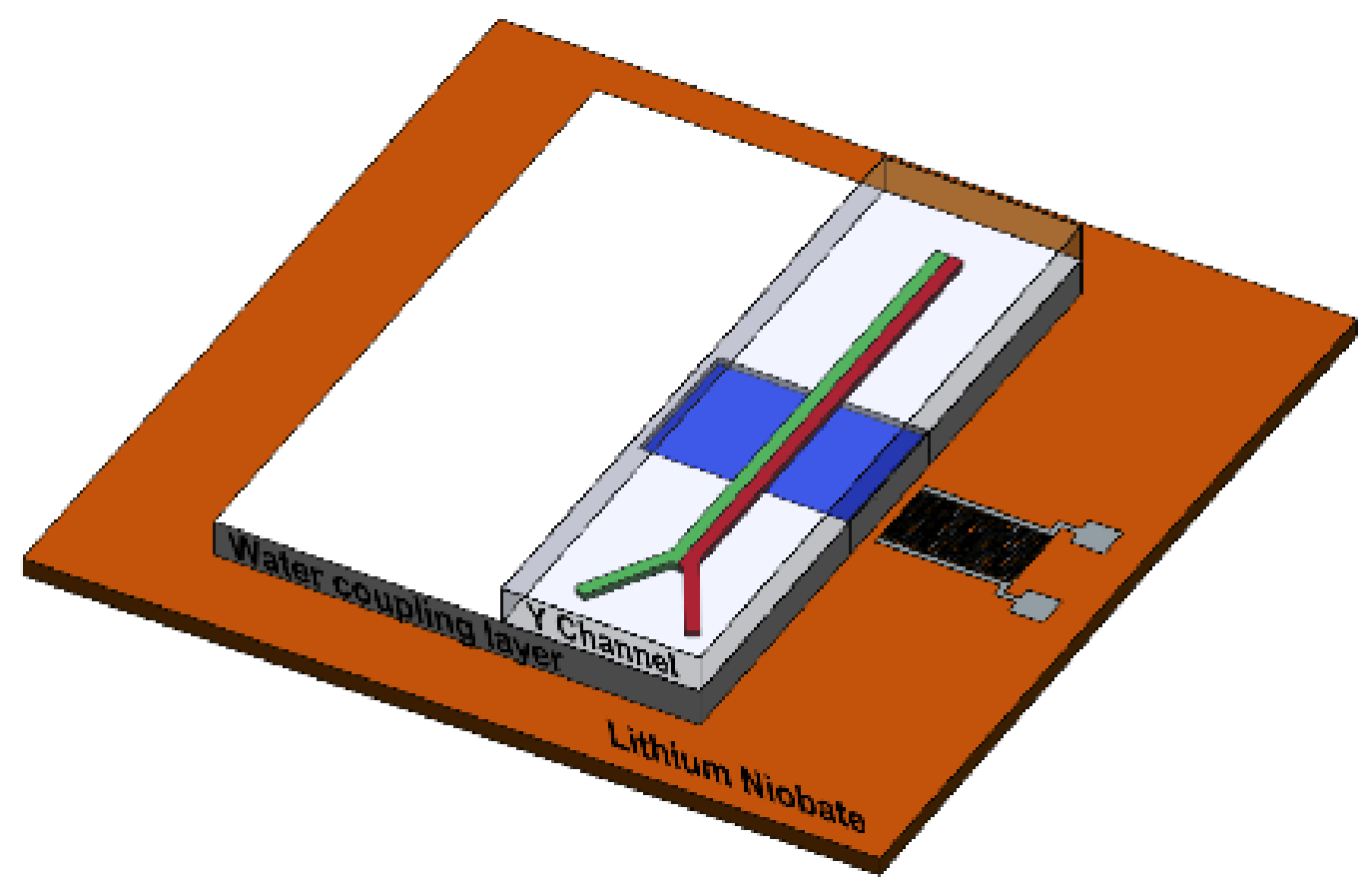

Figure 3.3: Reusable SAW mixing schematic. The acoustic wave generated on lithium niobate $\left(\mathrm{LiNbO}_{3}\right)$ was transmitted through a coupling layer of water into the microchannel made of PDMS bonded to glass. The mixing channel has a width of $1.15 \mathrm{~mm}$ and a height of $200 \mu \mathrm{m}$.

\subsection{Experimental setup}

\subsubsection{Electrical setup}

The electrical signal was generated from a pulsed RF power amplifier, as a high voltage $(>30 \mathrm{~V}$ ) was required for mixing to take place. The pulsed RF input was generated using the Tektronix AFG3252 arbitrary function generator. A pulsed RF signal with a period of $T_{p}$ of $5 \mathrm{~ms}$ and $T_{O N}$ (duration for which the signal is turned on) of $1 \mathrm{~ms}$ was generated. The output signal from the function generator with an amplitude range of $100-200 \mathrm{mV}$ was amplified by a pulsed power amplifier (TOMCO) of $60 \mathrm{~dB}$ gain. The output of the pulsed power amplifier provided the input to the SAW device. 


\subsubsection{Coupling layer}

Water forms the coupling layer in our experiments. Other coupling layers like the UV cured epoxy (NOA61) and ultrasonic gel were also used. For reusing the SAW device for different tests, water was chosen as the coupling layer. To control the water layer thickness, a U-shaped reservoir made out of PDMS was used, which is opened in the front as shown in Fig 3.4. The opening in the front prevents further attenuation of SAW by the PDMS. A fixed volume of liquid is introduced by a pipette, which helps to control the water layer length. The thickness of the coupling layer is equal to the thickness of the PDMS reservoir block ( $1 \mathrm{~mm}$ thick).

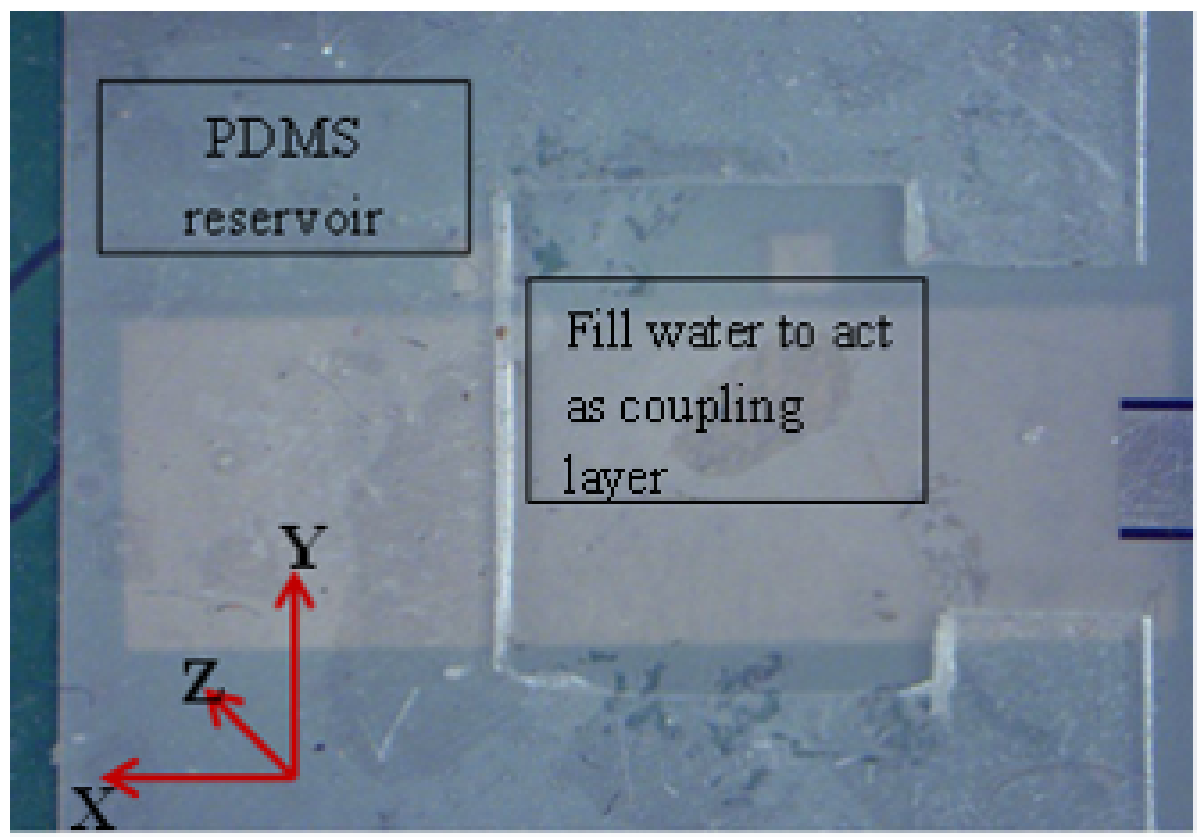

Figure 3.4: A block of PDMS functions as a reservoir to control the coupling layer thickness 


\subsubsection{Placement of the microfluidic channel}

Fig 3.5 shows the Y-shaped microchannel made on PDMS and bonded to glass. The channel is placed on top of the substrate via the coupling layer. The coupling layer length in the $\mathrm{X}$ direction (see Fig 3.4 for axis direction) is controlled by the placement of the microchannel, which was approximately $1.5 \mathrm{~mm}$ for the study. The layout and the dimensions of the microfluidic channel that was used for the experiment is shown in Fig 3.6. PDMS attenuates the acoustic wave, hence the PDMS was trimmed to reduce the attenuation.

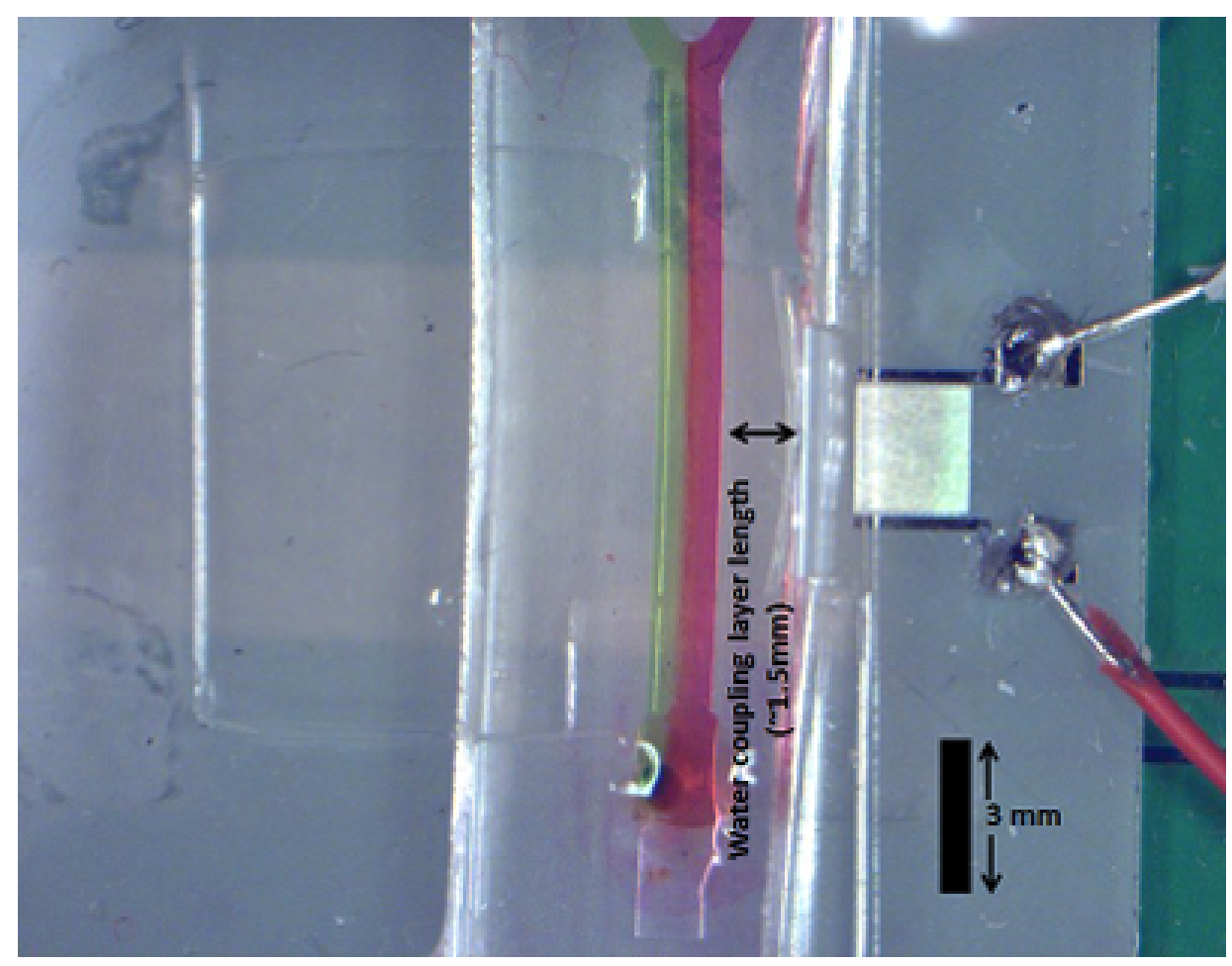

Figure 3.5: Microfluidic $Y$ channel placed on top of the substrate via coupling layer

\subsubsection{Microfluidic setup}

The mixing effect due to the SAW disturbance was visualised using CMOS video camera (INFINITY1). Food dyes of red and green colours were introduced into 
the microchannel at a flow rate of $1 \mu \mathrm{L} / \mathrm{min}$. The flow rate was controlled by syringe pumps (NE-1600, New Era, USA).

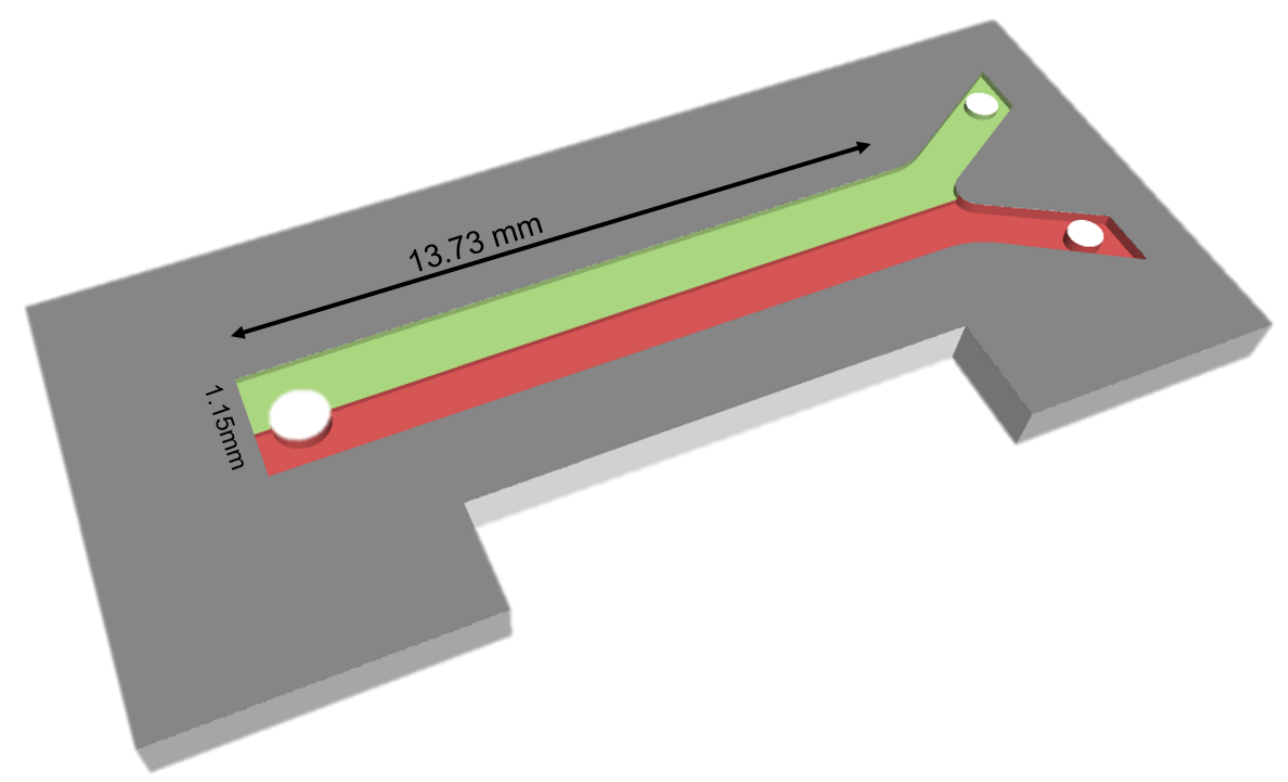

Figure 3.6: Y shaped microchannel on PDMS. The edge facing the IDT is trimmed to reduce the SAW attenuation

\subsubsection{Mixing efficiency calculation}

The mixing efficiency $(\sigma)$ was calculated by extracting the pixel intensities $I_{\text {unmix }}$ before mixing, $I_{i}$ obtained with the acoustic wave induced mixing and $I_{\text {mix }}$ after complete mixing [122] as follows:

$$
\sigma=1-\operatorname{sqrt} \frac{1}{N} \sum_{i=1}^{N} \sigma_{\mathrm{i}}^{2}
$$

where $\sigma_{\mathrm{i}}=\frac{I_{\mathrm{i}}-I_{\text {mix }}}{I_{\text {unmix }}-I_{\text {mix }}}$ and $N$ is the total number of pixels. The value of $\sigma$ varies from zero for non-mixing to one for complete mixing. Please note that during the mixing test, the experimental setup (mixing platform and the channel placement) remain unchanged. By the laminar nature of the flow, the two liquids initially flowed separately without significant mixing as seen in Fig 3.7(a). Upon initializing the SAW, mixing oc- 
curred between the two streams of fluid, as shown in Fig 3.7(b). The two dyes were then homogenously mixed externally and flowed through the microchannel as shown in Fig 3.7(c), this was representative of the completely mixed state. The region of interest (ROI) was selected to be within the SAW aperture region, shown in Fig 3.7(a).

(a)

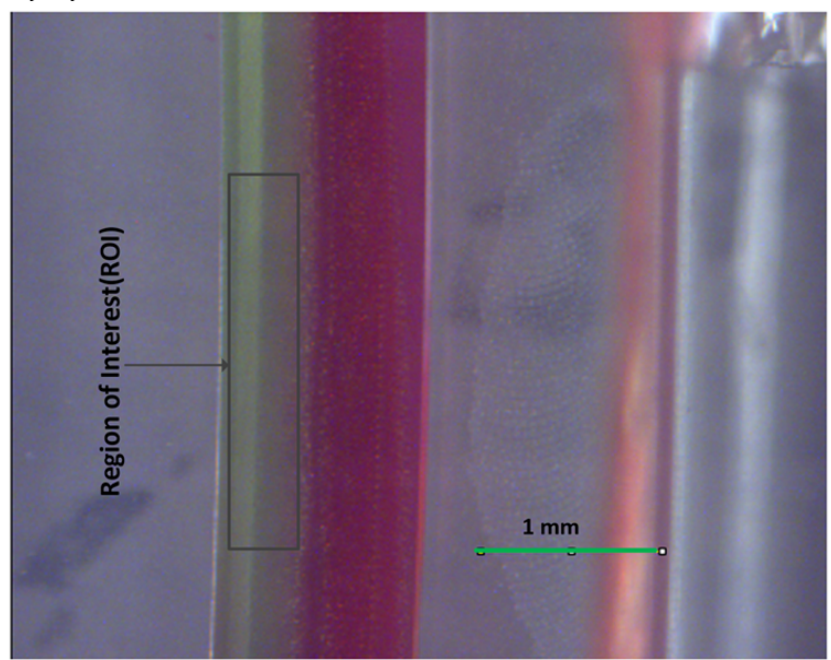

(b)

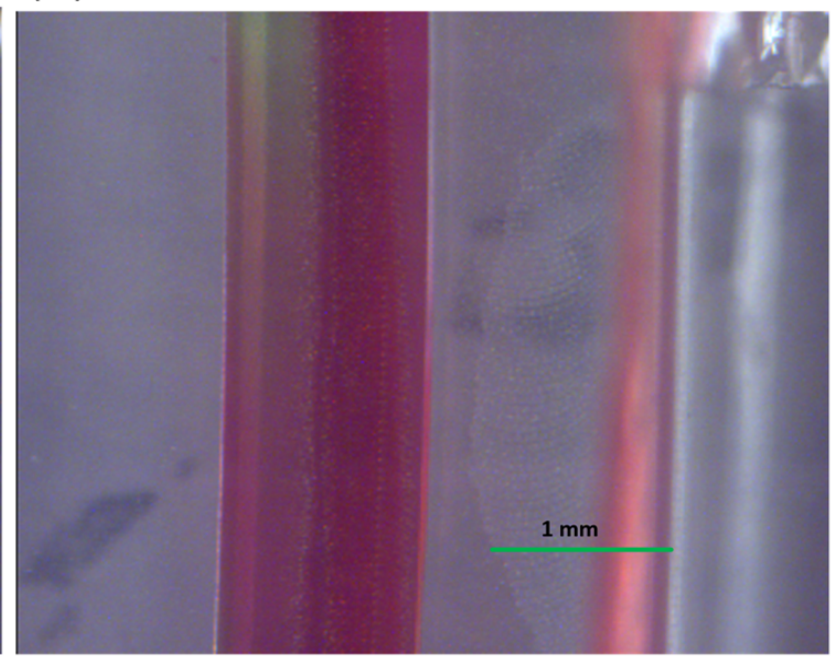

(c)

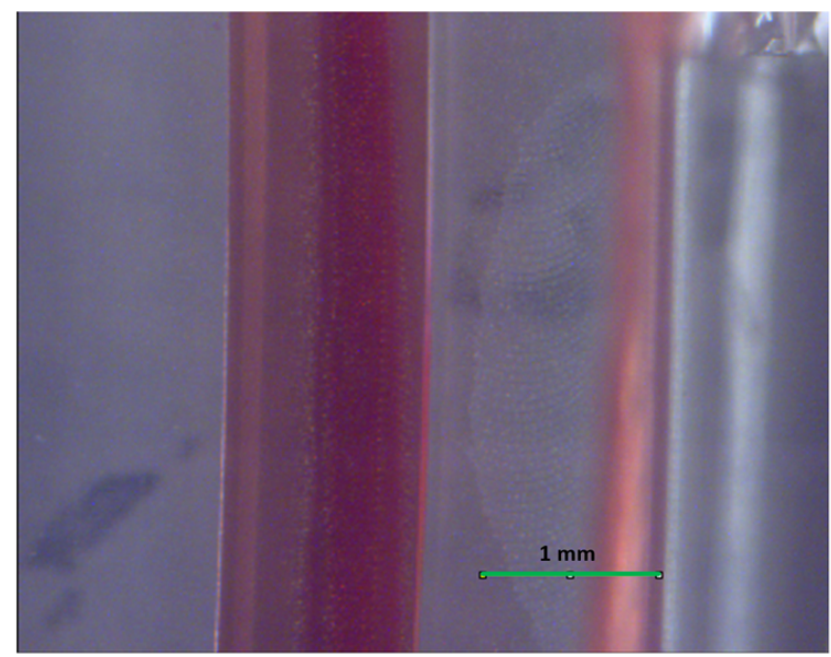

Figure 3.7: Images of the SAW mixing experiment (a) unmixed liquids at the beginning of the experiment, (b) fluids mixed using SAW (c) Homogenously mixed liquids where the two dyes were mixed externally to form the homogenously mixed solution. 


\subsection{Results and discussion}

To calculate the efficiency using Eq. 3.1, the pixel values (corresponding to the intensity) was extracted using the ImageJ software [123]. An average of 20,000 pixels ( $\mathrm{N}$ in Eq. 3.1) in the ROI was used to calculate the efficiency.

\subsubsection{Transient behaviour of mixing}

Fig 3.8 shows the transient response of the mixing phenomena. The increase in mixing efficiency can be observed immediately, before saturating at a value dependent on the voltage level. The experimental result demonstrated that the mixing be-

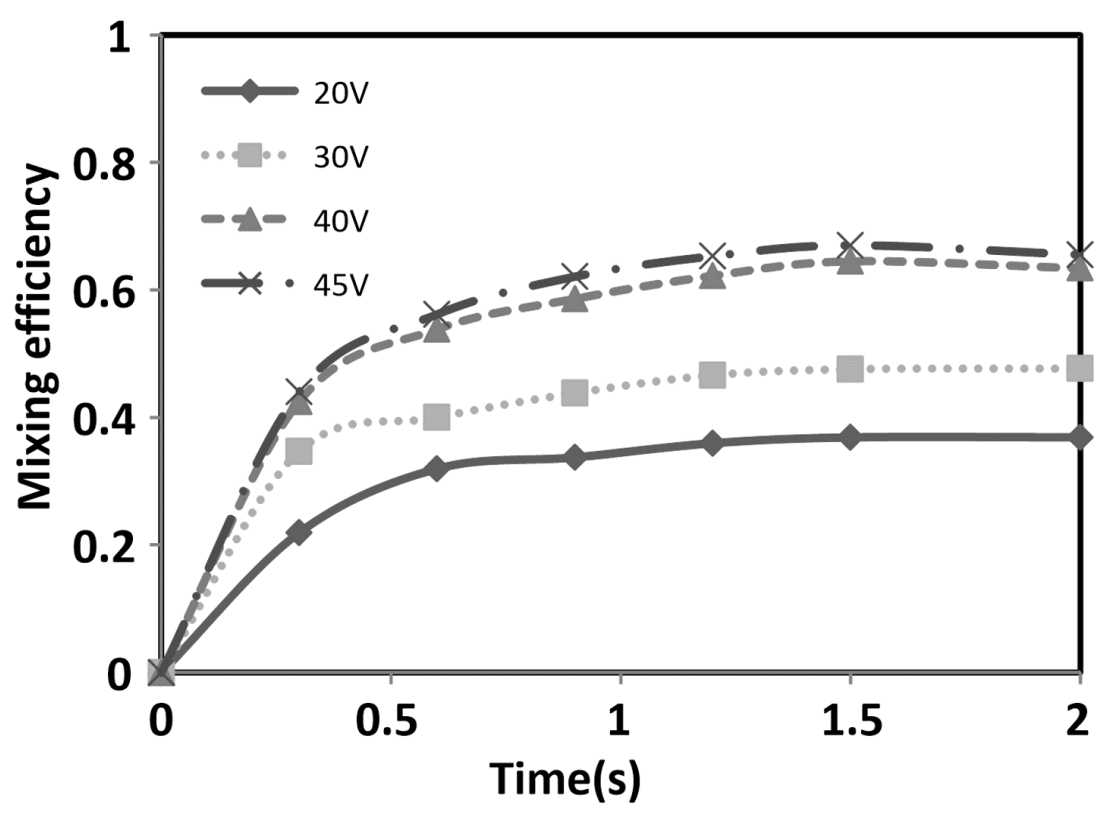

Figure 3.8: Transient mixing phenomena. The mixing achieved saturation within $1.5 \mathrm{~s}$.

haviour saturated within a time of 1.5 seconds. $70 \%$ efficiency at a voltage input of 45 $V$ was observed in $1.5 \mathrm{~s}$. Hence, in this work we extract the frame at $1.5 \mathrm{~s}$ to calculate the acoustic wave induced mixing efficiency. 


\subsubsection{Effect of voltage on mixing}

To examine further the influence of voltage on mixing, the voltage was varied from $20 \mathrm{~V}$ to $60 \mathrm{~V}$. The mixing efficiency was calculated at each voltage step. Fig 3.9 shows the mixing efficiency with different voltage for the device working at $50 \mathrm{MHz}$. Maximum efficiency of $80 \%$ was attained with an input voltage of $50 \mathrm{~V}$. The mixing efficiency graph demonstrated two trends with increasing voltages. At lower voltages, the mixing efficiency increased with voltage rapidly following a second order polynomial response. At higher voltages (around $50 \mathrm{~V}$ ), the mixing efficiency saturated. The interaction of the acoustic wave with fluid generates acoustic streaming [124]. As the wave is applied in a direction perpendicular to the liquid flow direction, acoustic streaming forces a mass movement in an orthogonal direction of flow and thus rapidly induces mixing. Acoustic streaming is a nonlinear second-order phenomenon. As reported by Nguyen and White [125], the acoustic streaming velocity is proportional to the square of the applied voltage. Mixing efficiency, which is proportional to the acoustic streaming velocity, thus increases with the square of the voltage. The experiment observation obtained is in line with this trend. At higher voltages, the nonlinearity of the acoustic field in the fluid causes the saturation of the acoustic streaming velocity [126,127]. In this study, the mixing efficiency saturated beyond an applied voltage (50 V) which supports prior research findings.Due to saturation in the level of mixing, the input power need not be increased beyond $50 \mathrm{~V}$. Any further increase would reduce the power conversion efficiency

\subsubsection{Effect of coupling layer thickness on mixing efficiency}

The acoustic mixing efficiency is dependent on the thickness of the coupling layer. The effect of varying thickness on the efficiency was examined experimentally. The experimental observation in 3.11 shows that a larger coupling layer thickness gives rise to a higher mixing efficiency The acoustic impedance of the coupling layer 


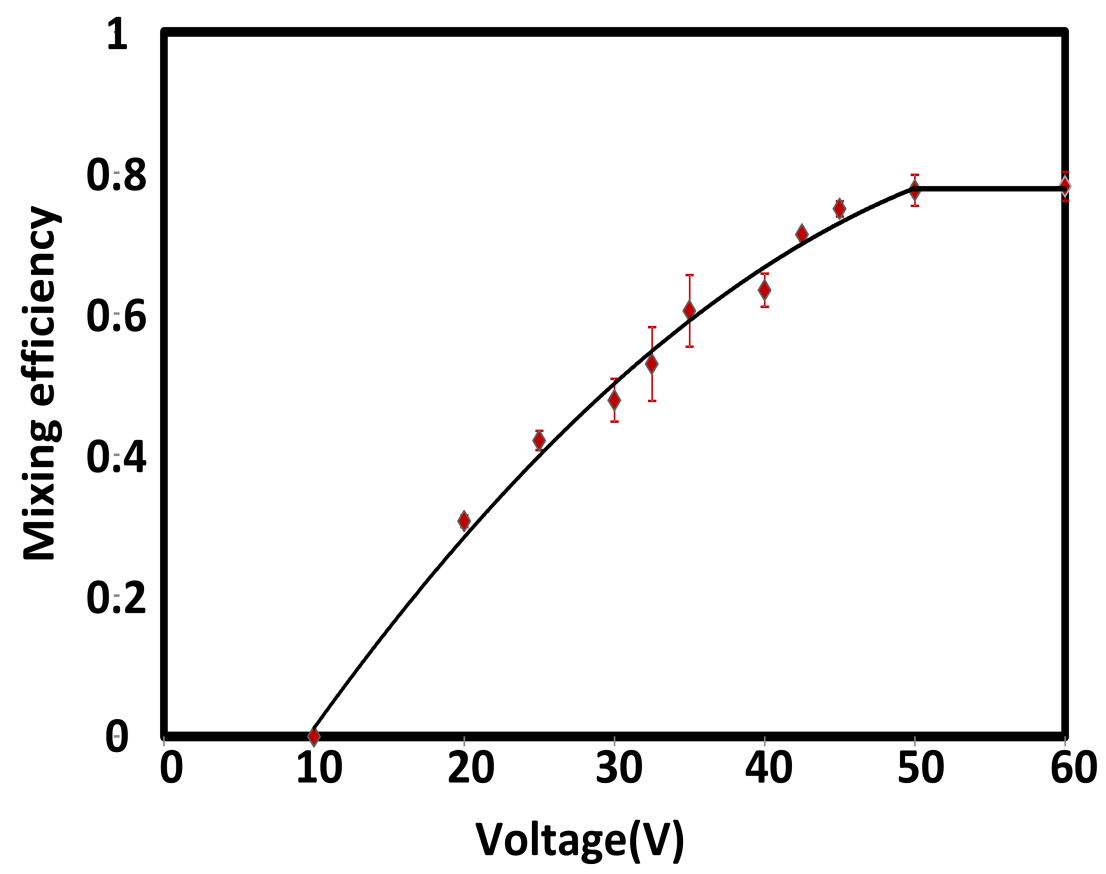

Figure 3.9: Mixing efficiency as a function of voltage. The error bars show the mixing efficiency variation from the mean value. The solid line shows a second order polynomial fit for voltages less than $50 \mathrm{~V}$ and further saturates at higher voltages.

prevents total reflection of the incoming wave from the $\mathrm{LiNbO}_{3}$ substrate. The wave diffracts into the water coupling layer from the SAW device at an angle of $22.1^{0}$. However, on passing through the coupling layer of a certain thickness $(L)$, the wave gets attenuated [128]. Fig 3.10 shows the wave amplitude through the various layers to determine the power transmission coefficient $\left(T_{r}\right)$. The power transmission coefficient can be calculated from [129]:

$$
T_{r}=4 r_{13} /\left(r_{13}+1\right)^{2}\left[1-\left(r_{23}^{2}-1\right)\left(r_{12}^{2}-1\right)\left(\sin K_{2} L\right)^{2} /\left(r_{13}+1\right)^{2}\right]
$$

with $r_{i j}=\rho_{j} c_{j} / \rho_{i} c_{i}$, where $\rho$ c represents the characteristic acoustic impedance with $c$ and $\rho$ denoting the wave velocity and density respectively in the medium. $L$ denotes the coupling layer thickness. The parameter of Eq. 3.2, other than the sinusoidal function remains unchanged for the experiment. The power transmission coefficient $\left(T_{r}\right)$ is directly proportional to the square of the sinusoidal function. For the operating fre- 


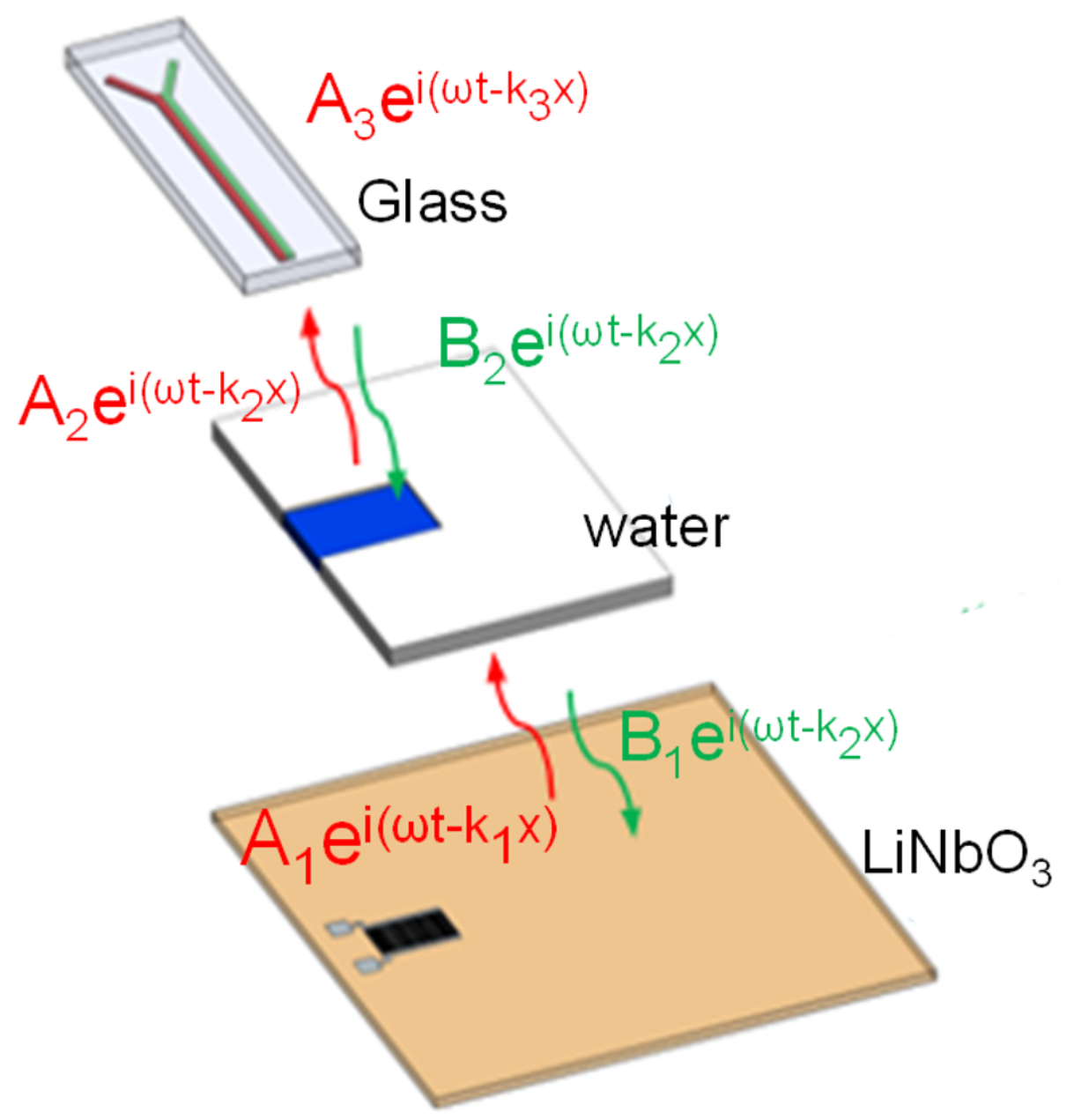

Figure 3.10: Wave transmission through the various layers. $A_{i}$ and $B_{i}$ represent the transmitted and reflected wave amplitude respectively at each layer. The terms in red denote the incident $\left(A_{1}\right)$ and transmitted waves $\left(A_{2}, A_{3}\right)$. The reflected waves $\left(B_{1}, B_{2}\right)$ are represented in green. The exponential part shows the phase dependence of the wave on the acoustic wave frequency $(\omega)$ and wavenumber $(k)$.

quency of $50 \mathrm{MHz}$, the square of sine function gives values of $8.6 \times 10^{-8}, 0.71$ and 0.75 for the coupling layers of thicknesses $0.6 \mathrm{~mm}, 0.7 \mathrm{~mm}$ and $0.8 \mathrm{~mm}$ respectively. Thus, $T_{r}$ increases for thicknesses in the order of $0.8 \mathrm{~mm}, 0.7 \mathrm{~mm}$ and $0.6 \mathrm{~mm}$. The experimental results shown in Fig 3.11 thus confirms the theoretical model. An optimum coupling layer thickness can be designed to reduce the attenuation. 


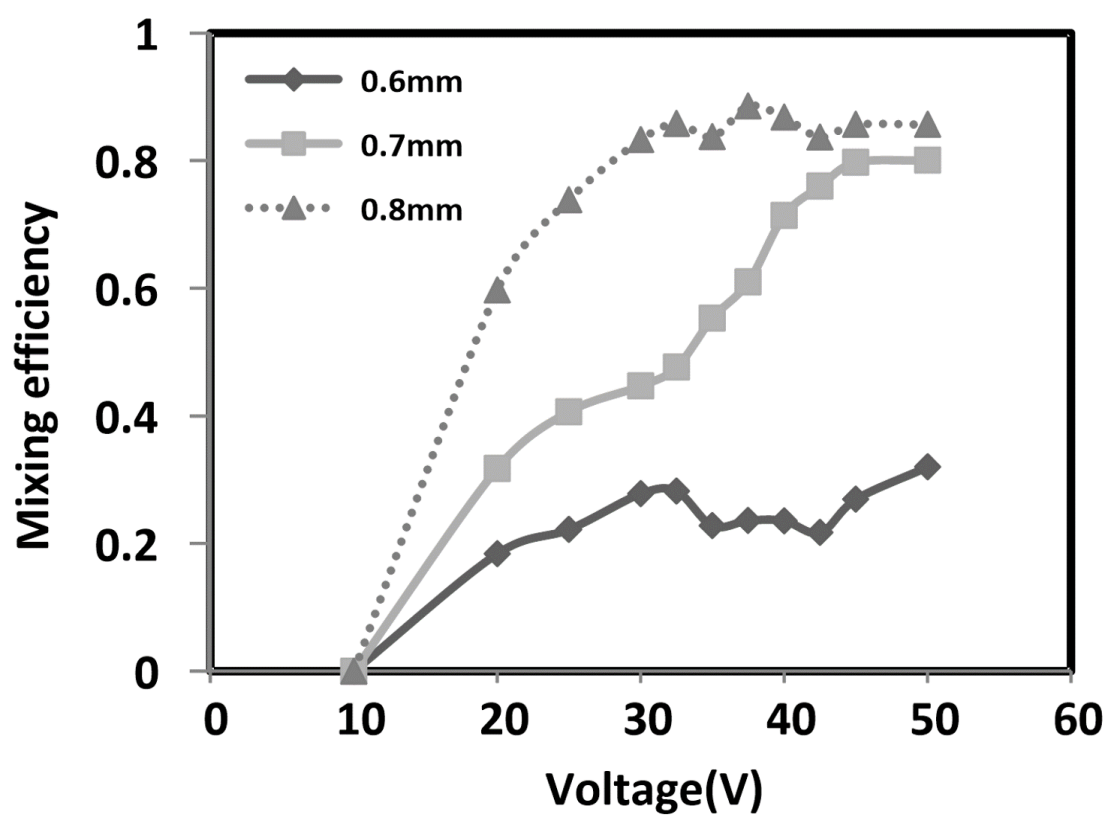

Figure 3.11: Mixing efficiency variation with coupling layer thickness

\subsubsection{Effect of frequency on mixing}

The SAW devices designed for frequencies of $50 \mathrm{MHz}$ and $100 \mathrm{MHz}$ were used to study the effect of frequency on the mixing efficiency. A coupling layer thickness of $1 \mathrm{~mm}$ was used for the experiment. As observed experimentally and shown in Fig 3.12, the SAW device working at $100 \mathrm{MHz}$ has a higher mixing efficiency than $50 \mathrm{MHz}$ at lower voltages. However, at higher voltages, the mixing efficiency reaches a maximum as mentioned in the effect of voltage, and both the devices deliver nearly equal mixing efficiency. The analysis for the effect of frequency is twofold: SAW power $\left(P_{s}\right)$ is generated on the substrate at different frequencies and delivered into the fluid through the various transmission layers. The power produced by the transducer is given by [90]:

$$
P_{s}=G_{a}|V|^{2} / 2
$$

where $G_{a}$ is the IDT conductance and $V$ is the applied electrical potential.As the IDT is bidirectional, only half of the wave power is delivered into the microfluidic chip. $G_{a}$ 


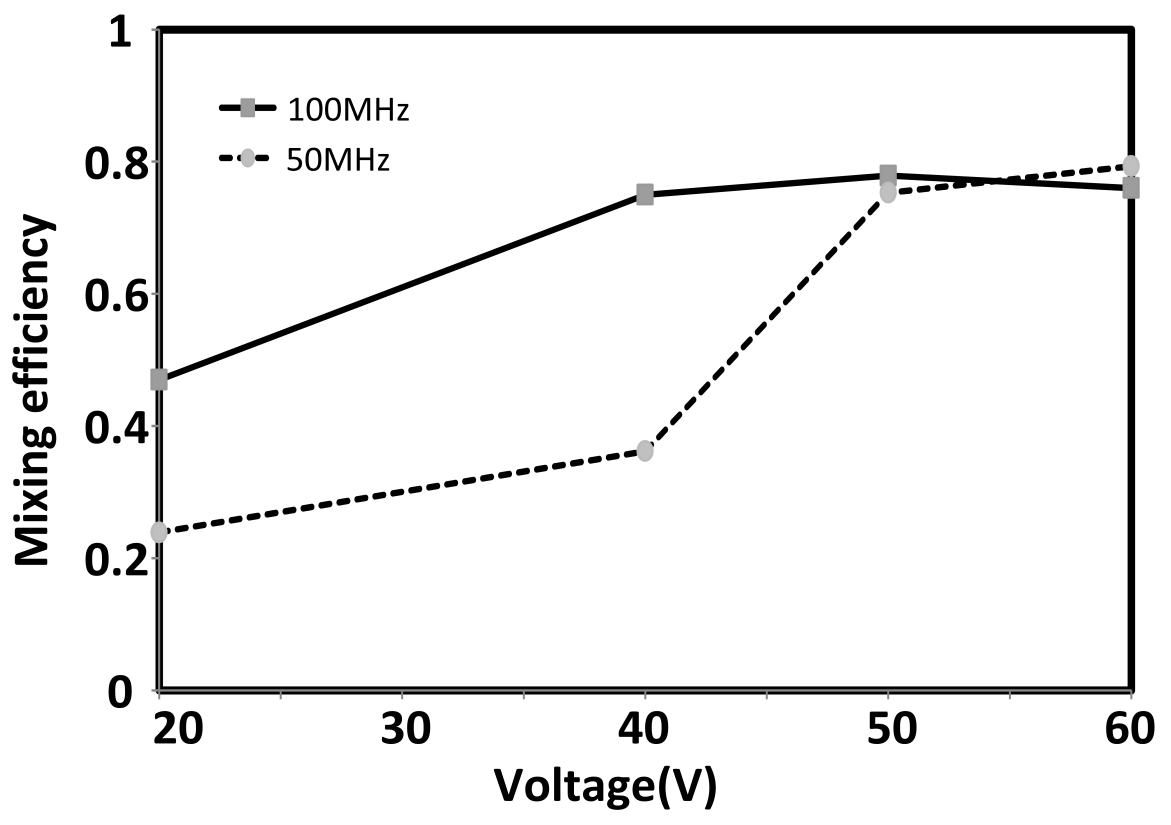

Figure 3.12: Mixing efficiency obtained for SAW devices at $50 \mathrm{MHz}$ and $100 \mathrm{MHz}$.

is proportional to the frequency and the aperture of the IDT. The power generated by the transducer for the two frequencies is:

$$
P_{s}(50 M H z)=0.533 P_{s}(100 M H z)
$$

Hence, the acoustic wave power generated by the $100 \mathrm{MHz}$ SAW device is approximately twice the power delivered by the $50 \mathrm{MHz} S A W$ transducer. The acoustic wave generated passes through the various layers before it reaches the microchannel as shown in Fig 3.13. As the wave passes through the thin water layer, it undergoes an attenuation of $0.55 \mathrm{~dB}$ and $2.3 \mathrm{~dB}$ for the $50 \mathrm{MHz}$ and $100 \mathrm{MHz}$ devices respectively. The longitudinal wave generated in the fluid reaches the water-glass (bottom) interface at an incident angle $\left(\theta_{W}\right)$ of $22.1^{\circ}$. The compressional waves from water excite Lamb waves in the thin glass plate of thickness $0.15 \mathrm{~mm}$. As discussed previously by Hodgson et al. [107], higher order antisymmetric waves are generated on the glass plate. The dispersion curve for the glass $[130,131]$ shows that at a frequency of 50 
$\mathrm{MHz} \mathrm{A}_{1}$ mode is excited. The $100 \mathrm{MHz}$ incident acoustic wave excites the $\mathrm{A}_{1}, \mathrm{~S}_{1}$ and $A_{2}$ Lamb wave modes. For the excited modes, the phase velocity of the normal wave is less than the speed of the longitudinal waves c $(5960 \mathrm{~m} / \mathrm{s})$ and close to the transverse velocity $b(3200 \mathrm{~m} / \mathrm{s})$ on the plates. The displacements for mode $A_{n}$ in this case are [132] given below. (Refer to Appendix A for details on the derivation of Eq. 3.5):

$$
\begin{array}{r}
u_{1} \cong\left(n+\frac{1}{2}\right) \frac{\pi}{h} A\left\{-\sin \left[(2 n+1) \pi \frac{x_{2}}{2 h}\right]+2(-1)^{n} \frac{\sinh \left(\chi k x_{2}\right)}{\sinh (\chi k h)}\right\} \\
u_{2} \cong i K A\left\{\cos \left[(2 n+1) \pi \frac{x_{2}}{2 h}\right]+(2 n+1)(-1)^{n} \frac{\pi \chi}{k h} \frac{\chi k x_{2}}{\chi^{h}}\right\}
\end{array}
$$

where $u_{1}$ and $u_{2}$ are the longitudinal and vertical (transverse) components of the mechanical displacement. The plate thickness along the $x_{2}$ direction is equal to $2 \mathrm{~h}$ and $\mathrm{n}$ represents the mode number. Computation of the transverse component of displacement $\left(\mathrm{u}_{2}\right)$ on the surface of the plate $\left(\mathrm{x}_{2}=h\right)$ shows that the magnitude of second order mode $\left(A_{2}\right)$ is about three times the magnitude of the first order mode $\left(A_{1}\right)$. The second order mode excited in the $100 \mathrm{MHz}$ excitation generates larger displacement, consequently improves mixing efficiency. The sound wave transmitted through the glass plate placed between two liquid media, can also be analysed quantitatively by the Eq. 3.6 obtained from Brekhovskikh [133]. (Refer to Appendix B for the derivation of Eq. 3.6).

$$
\begin{array}{r}
W=2 N /\left[2 M+i\left(M^{2}-N^{2}-1\right)\right] \\
M=\left(Z_{2} / Z_{1}\right) \cos ^{2} 2 \gamma_{2} \cot P+\left(Z_{2 t} / Z_{1}\right) \sin ^{2} 2 \gamma_{2} \cot Q \\
N=Z_{2} \cos ^{2}\left(2 \gamma_{2}\right) / Z_{1} \sin P+Z_{2 t} \sin ^{2}\left(2 \gamma_{2}\right) / Z_{1} \sin Q \\
P=k_{2}(h / 2) \cos \theta_{2} \\
Q=\kappa(h / 2) \cos \gamma_{2}
\end{array}
$$

where $K_{2}, \kappa_{2}$ are the wavenumbers of the acoustic wave for the longitudinal and transverse component respectively in glass; $Z_{2}, Z_{2 t}, Z_{1}$ denotes the acoustic impedance for the longitudinal, transverse wave in glass, and the compressional wave in water re- 
spectively; $\theta_{2}, \gamma_{2}$ are the angle of incidence for the longitudinal and transverse wave in glass. The longitudinal wave in glass undergoes total internal reflection and the wave only resides on the surface. The angle of incidence of the longitudinal wave is represented in the complex form. We set $\theta_{2}=\pi / 2+i \zeta$, with $\zeta=0.9579$, using this $\sin \theta=\cosh \zeta, \cos _{\theta}=-i \sinh \zeta$. The transmission coefficient (power- $\left|W^{2}\right|$ ) for the $50 \mathrm{MHz}$ and $100 \mathrm{MHz}$ acoustic wave is $0.155(-8 \mathrm{~dB})$ and $0.2916(-5.35 \mathrm{~dB})$ respectively. The quantitative results align with the qualitative results obtained above, with the $100 \mathrm{MHz}$ excitation conveying larger power to the fluid above compared to the $50 \mathrm{MHz}$ acoustic wave excitation. From the reflection coefficient of the device shown in Fig 3.2, the $50 \mathrm{MHz}$ and $100 \mathrm{MHz}$ devices have a reflection coefficient of $0.794 \%$ and $15 \%$ respectively. Thus, the transmission coefficients for the two devices are -0.034 $\mathrm{dB}$ and $-0.71 \mathrm{~dB}$. There is an additional reduction of power by $-3.01 \mathrm{~dB}$ due to the bidirectional nature of the IDTs. Hence, summing up the various losses, the total coupling attenuation of power was $11.594 \mathrm{~dB}$ and $11.37 \mathrm{~dB}$ for the $50 \mathrm{MHz}$ and $100 \mathrm{MHz}$ devices respectively. The transmitted power is equal to the generated power by the SAW device minus the attenuated power through various layers. Since the generated power is almost 2 times higher in the $100 \mathrm{MHz}$ device when compared to the $50 \mathrm{MHz}$ device, the acoustic power delivered to the fluid is two times higher in the SAW device at $100 \mathrm{MHz}$ when compared to the $50 \mathrm{MHz}$. Hence the mixing efficiency is higher when excited by a $100 \mathrm{MHz}$ SAW device. Thus, the numerical computation shows that the power transmitted by the $100 \mathrm{MHz}$ device was higher than the $50 \mathrm{MHz}$ device.

\subsubsection{Acoustic heating}

Temperature rise changes the acoustic impedance of various layers and can hinder the model presented in Sec 3.4.3 for calculating the power transmission coefficient. To justify the validity of the model, we performed the following experiment to measure the temperature. The temperature was recorded at the $\mathrm{LiNbO}_{3}$ surface for a time-period of 20 seconds using a Fluke DT-610B thermometer. The observation 


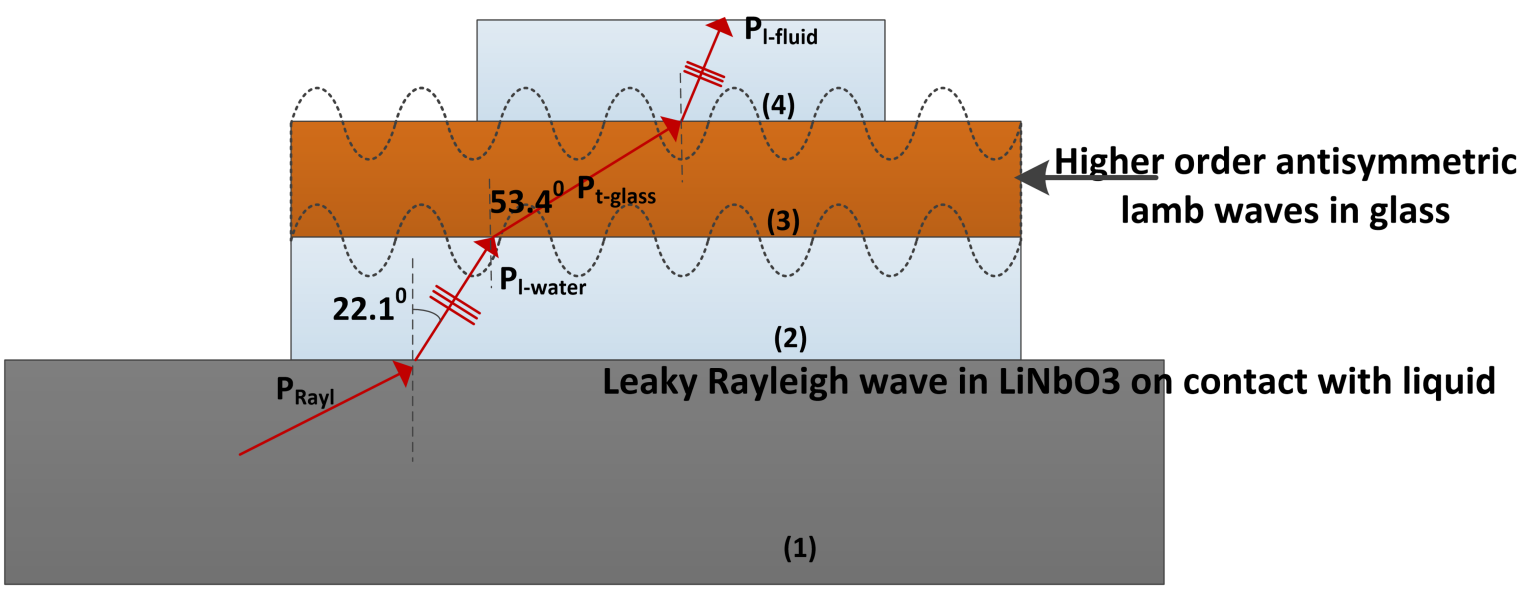

Figure 3.13: Acoustic Wave transmission through the various layers, showing the waves and its refracting angles at each layer. Medium 1, 2, 3 and 4 are the piezoelectric lithium niobate substrate, water coupling layer, glass and fluid within the microchannel respectively. $P_{l-\text { water }}$ represents the longitudinal wave in water, at an incident angle of $22.1^{0} ; P_{t-g l a s s}$ is the transverse wave in the glass with a transmission angle of $53.4^{\circ}$, the longitudinal component undergoes total internal reflection and will have the character of surface waves.

points (P1, P2 and P3) were chosen along the centre line of the SAW aperture as shown in Fig 3.14. Different Voltages (25V, $35 \mathrm{~V}$ and $45 \mathrm{~V})$ were also applied for the experiment. Fig 3.15 shows the experimental results for $20 \mathrm{~s}$. It indicates that for 20 $\mathrm{s}$, the temperature rises only from $24.35^{\circ} \mathrm{C}$ to $39.05^{\circ} \mathrm{C}$ (corresponding to $45 \mathrm{~V}$ and position $\mathrm{P} 1$ ). The mixing efficiency in our experiment was calculated at $1.5 \mathrm{~s}$. The temperature rises by a maximum of $0.8^{\circ} \mathrm{C}\left(24.35^{\circ} \mathrm{C}\right.$ to $\left.25.15^{\circ} \mathrm{C}\right)$ for the period of $1.5 \mathrm{~s}$. Considering the dependence of the velocity of sound in water [134] and density [135] as a function of temperature, a $0.16 \%$ change in the acoustic impedance is obtained. This temperature rise is considered negligible and is unlikely to cause a substantial amount of shift in the acoustic impedance. Hence, the Kinsler approximation model discussed in sec 3.4.3 is appropriate. The temperature increase is also not substantial to cause any heating of the liquid. 


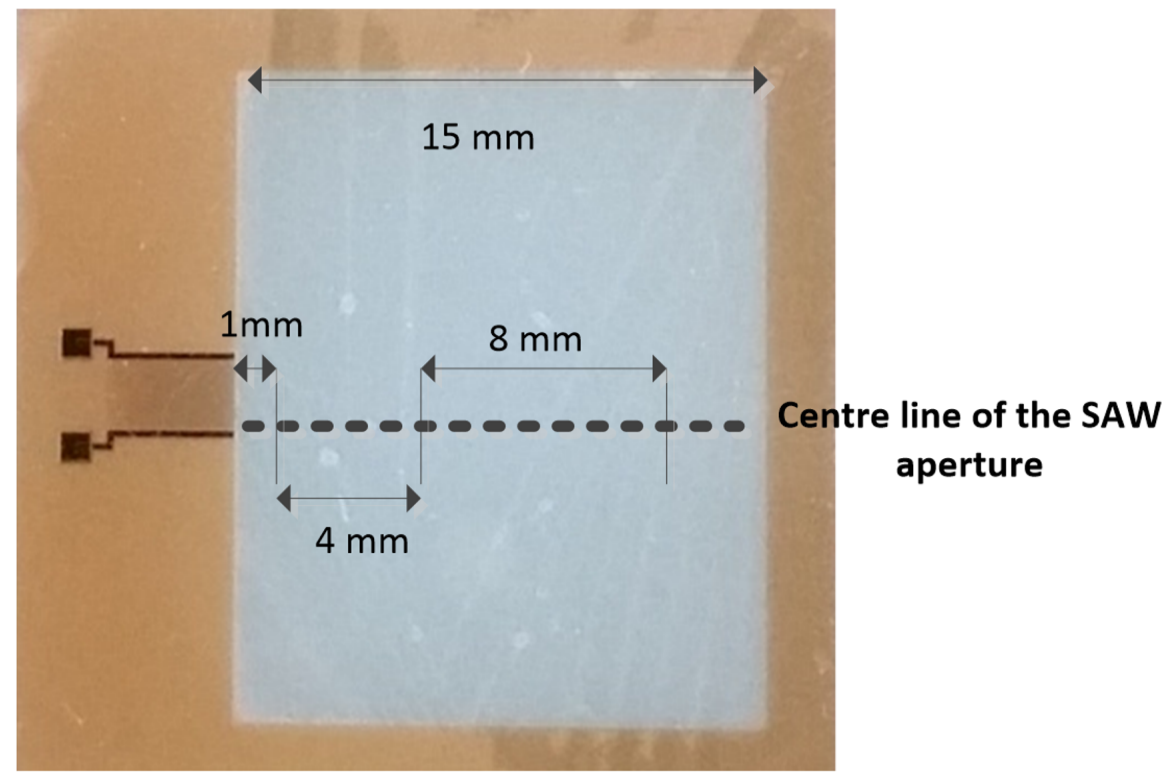

Figure 3.14: Positions along the SAW device for measuring the temperature. Three points were chosen along the centre line of the SAW aperture at distance of $1 \mathrm{~mm}$ $(\mathrm{P} 1), 5 \mathrm{~mm}(\mathrm{P} 2)$ and $13 \mathrm{~mm}(\mathrm{P} 3)$ from the IDT

\subsection{Concluding remarks}

In this chapter, we report the design, fabrication and characterization of a reusable microfluidic platform using SAW device. The Y-shaped microchannel was made from PDMS and bonded to a glass superstrate. It was coupled to the SAW, generated on the $\mathrm{LiNbO}_{3}$ substrate using water as an acoustic coupling layer. The platform was characterised using microfluidic mixing phenomenon. Two food-dye solutions were introduced into the microchannel and mixing efficiency was calculated. Mixing efficiency of $80 \%$ was observed within $1.5 \mathrm{~s}$ for an input signal of $50 \mathrm{~V}$. Experiment was conducted with varying coupling layer thickness to find the optimal thickness for maximum acoustic energy transmission. A SAW device at $50 \mathrm{MHz}$ gives rise to greater than $80 \%$ mixing efficiency for a coupling layer of $0.8 \mathrm{~mm}$ thickness. An analytical model formulated to predict the behaviour for different thicknesses substantiates the experimental findings. We then compared the mixing efficiency for two devices at 


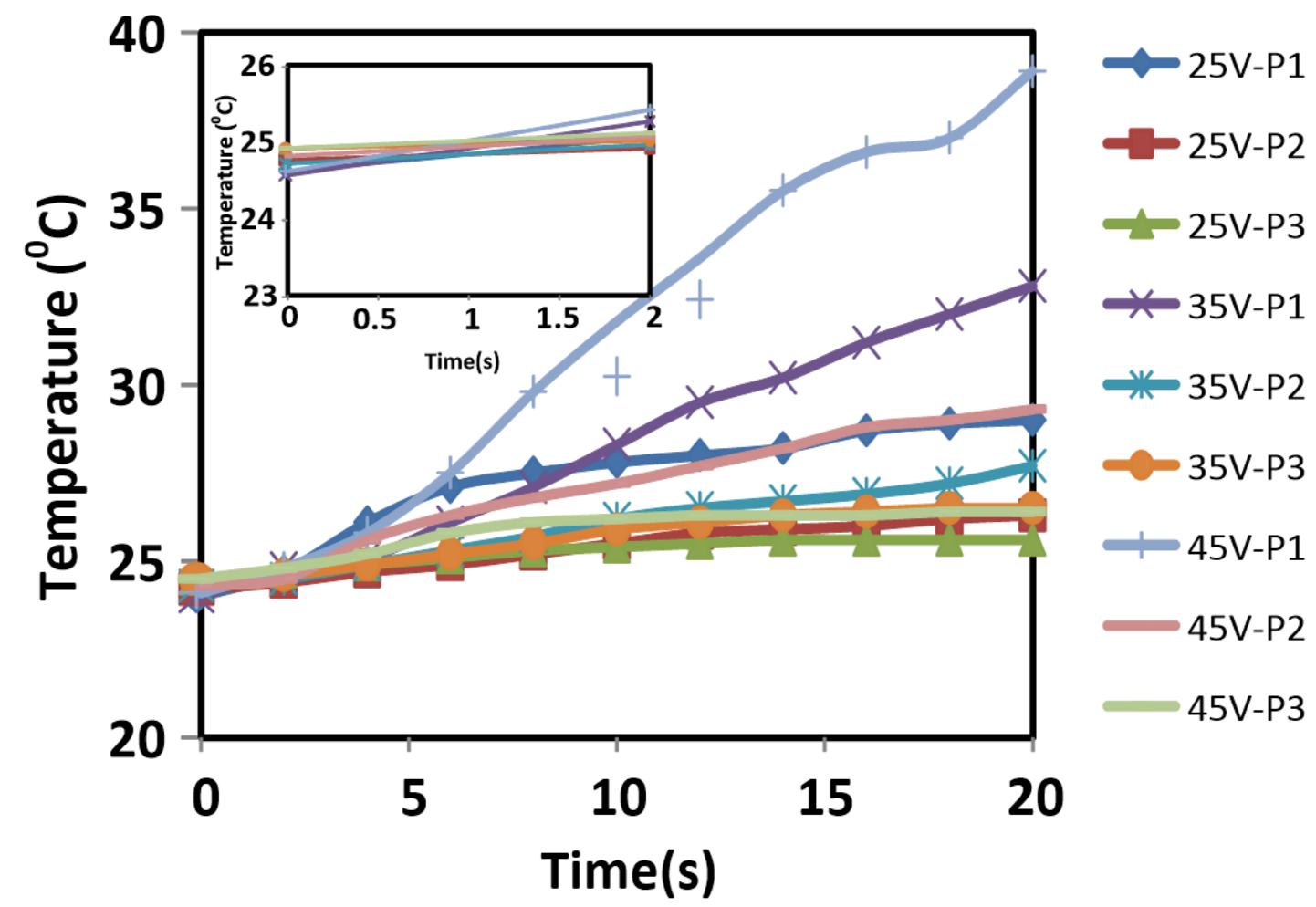

Figure 3.15: The temperature measured for a time period of $20 \mathrm{sec}$ with different voltages $(25 \mathrm{~V}, 55 \mathrm{~V}$ and $45 \mathrm{~V})$ at various positions as indicated in Fig 3.14. The inset shows the magnified view of the graph for a time period of $2 \mathrm{sec}$

frequencies of $50 \mathrm{MHz}$ and $100 \mathrm{MHz}$. The experimental result shows that the power transmission was higher for the $100 \mathrm{MHz}$ as compared to the $50 \mathrm{MHz}$, mainly due to the existence of higher order Lamb wave modes with larger displacement amplitudes. The results obtained provide useful design guidance for the selection of voltages, frequencies and the thickness of couplant layers for improving the acoustic energy transfer for various microfluidic manipulations. 


\section{Chapter 4}

\section{Photoacoustic induced surface}

\section{acoustic wave (SAW PA) sensor for}

\section{sensing analytes in a microfluidic}

\section{channel}

In this chapter, we report a novel sensor utilising the photoacoustic induced surface acoustic wave (SAW-PA) to detect the optomechanical properties of analytes inside a microfluidic channel. The longitudinal wave generated inside the microfluidic channel is mode-converted to a surface acoustic wave (SAW) and detected by the interdigital transducer (IDT). We present an analytical model to explain the mode conversion process and its attainable efficiency. We then demonstrate by experiments the usage of the sensor to investigate the optical and acoustical properties of standard dye solutions and gold nanoparticles. Subsequently, we elucidate the response mechanism of the sensor using detailed numerical FEM simulation. The simulation study has shown that the microfluidic channel on the substrate acts as an acoustic resonator with mul- 
tiple eigenmodes.PA signals generated inside the microfluidic channel resonates at these modes. The displacement profile on the piezoelectric surface confirmed that the pressure mode propagating parallel to the piezoelectric substrate has the highest mode conversion efficiency (longitudinal wave to SAW). This mode is sensitive to the microchannel dimensions. The study has shown that a SAW device matched to this mode /frequency could be used for improving the sensitivity of the sensor. These numerical observations supported with an experimental verification has led to an understanding of the SAW-PA sensor.

\subsection{Introduction}

SAW sensors have been used to determine the mechanical properties including viscosity and density of the fluids flowing through a channel between the electrodes $[136,137]$.On the other hand, optofluidic sensors have been explored to characterise the optical properties of the liquid samples [138-141] including absorptivity, refractive index and even mechanical properties of the fluids such as viscosity $[142,143]$.However, the measurement of the fluid viscosity requires introducing particles into the fluid as a probe and involves complex calculations. To achieve development of a comprehensive and concurrent (optical and mechanical properties) characterization pathway for diversified analytic samples, a sensor incorporating smart integration of light and sound is highly desirable. Recently, the development of new optical imaging techniques based on photoacoustic (PA) effect such as PA imaging and its combination techniques have been used extensively for sensing and imaging applications [144-147],especially in biomedicine [148, 149]. Details of the PA sensing technique was discussed in Chapter 2. Although PA sensing and imaging have demonstrated promising results in both anatomical and functional imaging of small animals in vivo, its applications in a compact microfluidic environment is rarely explored. This could be attributed to the inherent limitation of integrating the bulky ultrasound 
transducer on a small analytical microchip. In this chapter, we present the design and fabrication of a SAW-PA device, which performs PA sensing with a Rayleigh SAW device. The analyte sample for the investigation was loaded into a microfluidic channel made of polydimethylsiloxane (PDMS). The nanosecond pulsed laser focused on the fluid sample in the microchannel triggers PA waves, which couples into a Rayleigh SAW on the piezoelectric substrate for detection. This platform enables characterization of liquid samples by measuring both its optical and mechanical properties simultaneously. Furthermore, the usage of SAW as the receiver of the PA signal in this new design enables a couplant-free (water-free) PA signal sensing at a low cost and compact pathway. In this work, we first demonstrated the usage of the SAW-PA sensor to detect the optomechanical properties of organic dyes and plasmonic nanoparticles (NP), which represents an optically homogeneous and optically heterogeneous medium respectively for generating PA. The multitude of applications possible due to nanoparticles in scientific fields like medicine, pharmacy, nano and biotechnology, electronics, photonics and environmental science [150] highlights the importance of detecting NP and determining its morphology using a lab-on-chip device. The primary goal of the numerical modelling was to determine the frequency of the PA excited SAW and use the information in the design of the interdigital transducer (IDT), for increasing the sensitivity of the sensor. Besides the frequency determination, the chapter aims to elucidate: 1) The need of a narrow-band device to detect a wideband PA signal. 2) How do we efficiently convert a longitudinal acoustic wave to SAW without additional wedge structures as discussed in the previous chapter. Various groups $[86,87]$ performed work about the analytical and numerical models for PA generation in liquid. According to our knowledge, the study on the mode-conversion of PA generated longitudinal waves in fluids into SAW on piezoelectric substrates has not been done elsewhere. However, some early works were performed to study the laser-generated surface acoustic waves $[151,152]$ on solid materials. In this section, we prove that the laser-generated longitudinal wave in the microfluidic channel undergoes resonance at 
various modes. The frequency which excites surface waves at the highest amplitude is the pressure mode in the channel which propagates in a direction parallel to the piezoelectric surface. The derivation of the model is based on the coupling of multiple physics interfaces in COMSOL Multiphysics 4.4. The thermoacoustic module was used to solve for the acoustic degree of freedom inside the fluid, pressure propagation in the polydimethylsiloxane (PDMS) was modelled using pressure acoustics and the piezoelectric interface comprising of solid mechanics and electrostatics simulates the SAW propagation and electrical voltage from the IDT, respectively. The accuracy of the frequency predicted by the model has been validated by performing an experiment on the sensitivity of the SAW-PA sensor to IDTs at different centre frequencies. The contents of this chapter is based on our work published in $[153,154]$

\subsection{Materials and Methods}

\subsubsection{SAW device design and fabrication}

SAW device was designed and fabricated on a $128^{\circ} \mathrm{YX}$ lithium niobate $\left(\mathrm{LiNbO}_{3}\right)$ substrate with split-finger inter-digital transducer (IDT). Table 4.1 and Fig 4.1 provide the detailed dimensions of the SAW IDT and the frequency response (S11) of the SAW device (measured using E5061B ENA Series Network Analyzer) respectively. The resonant frequency of the device is at $12.9 \mathrm{MHz}$.

\begin{tabular}{|c|c|}
\hline Parameter & Values \\
\hline IDT width $(\lambda / 8$ in $\mu \mathrm{m})$ & 37.5 \\
\hline Number of IDT pairs & 25 \\
\hline IDT thickness $(\mathrm{nm})$ & 150 \\
\hline Aperture $(\mathrm{mm})$ & 4 \\
\hline
\end{tabular}

Table 4.1: SAW-IDT design parameters 


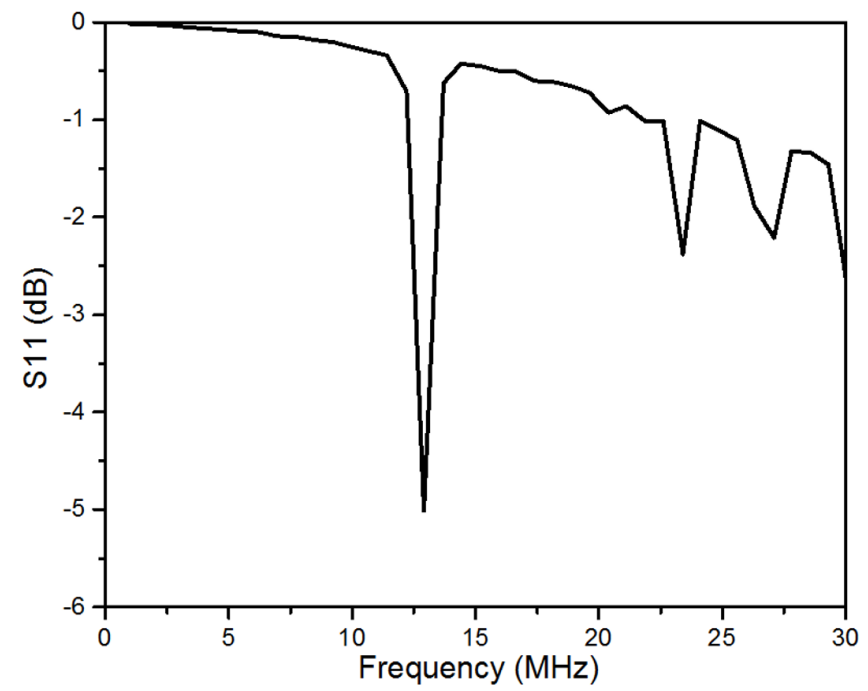

Figure 4.1: Frequency response (S11) of the SAW device measured using the network analyser. The resonant frequency of the device is $12.9 \mathrm{MHz}$.

\subsubsection{Design of microfluidic channel}

A Polydimethylsiloxane (PDMS) microfluidic channel of width $1.15 \mathrm{~mm}$, depth $150 \mu \mathrm{m}$ and length $15 \mathrm{~mm}$ was fabricated by soft lithography with through-holes punched for both inlet and outlet. The PDMS was trimmed along the width (sidewall thickness$\mathrm{T}_{\mathrm{s}}$ of $3 \mathrm{~mm}$ as shown in Fig 4.2) to reduce the PDMS attenuation of the acoustic wave. The PDMS was bonded to the substrate via oxygen plasma treatment (Inspiraz Technology). The PDMS and the $\mathrm{LiNbO}_{3}$ substrate were cleaned and exposed to oxygen plasma for 2 min. The PDMS microchannel was then aligned and bonded to the SAW substrate. The device was kept in an oven maintained at a temperature of $80{ }^{\circ} \mathrm{C}$ to strengthen the bond.

\subsubsection{Reagent and solutions}

We have selected aqueous solutions of two commonly used food colouring dyes and gold nanoparticles as plasmonic materials for analysis. The dyes, erythrosine (red) and brilliant blue were purchased from SigmaAldrich Singapore. Spherical 


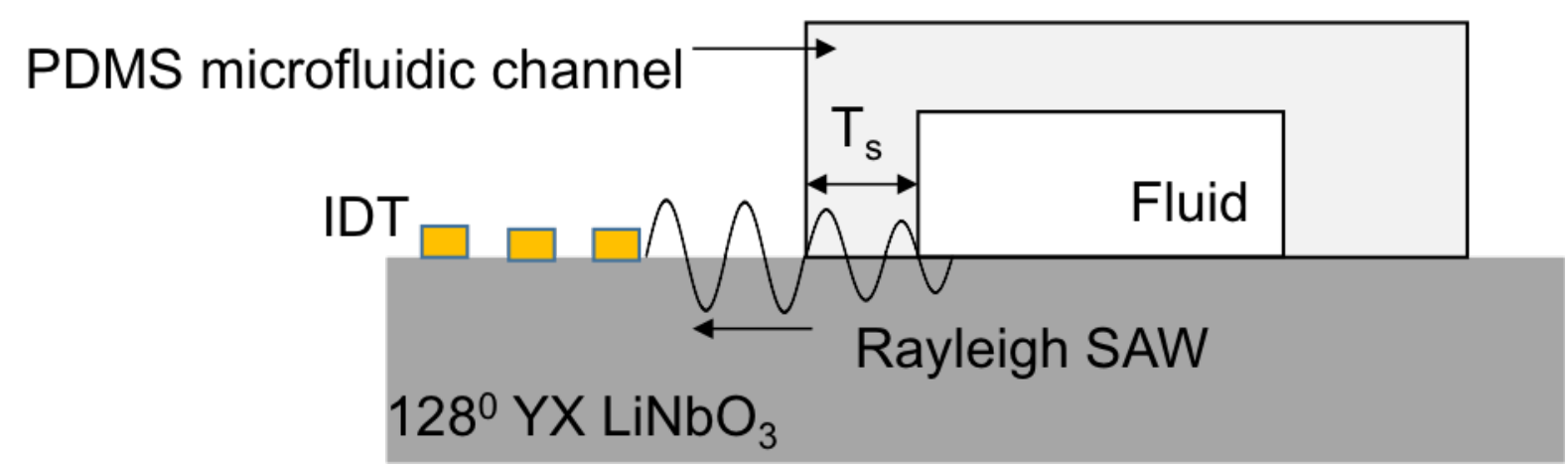

Figure 4.2: Schematic illustration of the PDMS microfluidic channel on the SAW substrate, where Ts denotes the side wall thickness

gold nanoparticles (GNPs) and gold nanorods (GNRs) were obtained from Nanopartz Inc., USA. All the solutions were prepared with millipore deionized (DI) water. UV/vis absorption spectra of the dyes and NPs were obtained using a Shimadzu UV-3600 UV-vis-NIR spectrometer.

\subsubsection{Experimental setup}

A nano-second pulsed laser light of $1 \mathrm{~mJ}$ pulse energy was focused on the microchannel within the aperture of the SAW device, to induce PA signal. The laserinduced PA signal within the fluid in the microchannel was converted to surface acoustic waves in the piezoelectric $\left(128^{\circ} \mathrm{YX} \mathrm{LiNbO}_{3}\right)$ substrate. The surface acoustic wave propagating on the substrate was detected by the IDT. Fig 4.3 shows the schematic representation of the device. Fig 4.4 illustrates the experimental configuration consisting of the optical interrogation units, surface acoustic wave device and the electrical measurement units in SAW-PA device. An optical parametric oscillator (OPO) pulsed laser with tunable wavelength $(410-2100 \mathrm{~nm}), 7 \mathrm{~ns}$ pulse-width, and $1 \mathrm{~mJ}$ pulse energy, was employed as the light source. After attenuation by the neutral density (ND) filter and focusing by the condenser lens, the light was illuminated on the microfluidic channel filled with liquid sample. A pre-amplifier (Panametrics 5077PR) with $59 \mathrm{~dB}$ gain was used to amplify the surface wave from the IDT, followed by data acquisition 
from an oscilloscope (WaveSurfer from LeCroy). Fig 4.5(a) shows individual components including split-finger aluminium metal IDT of thickness $150 \mathrm{~nm}$, which was deposited on the $128^{\circ} \mathrm{YX} \mathrm{LiNbO}_{3}$. Metal pad of thickness $500 \mathrm{~nm}$ was used to connect the IDT to the external electrical circuits. Fig 4.5(b) shows the vertical alignment of fluidic compartment made of PDMS bonded to the piezoelectric substrate.

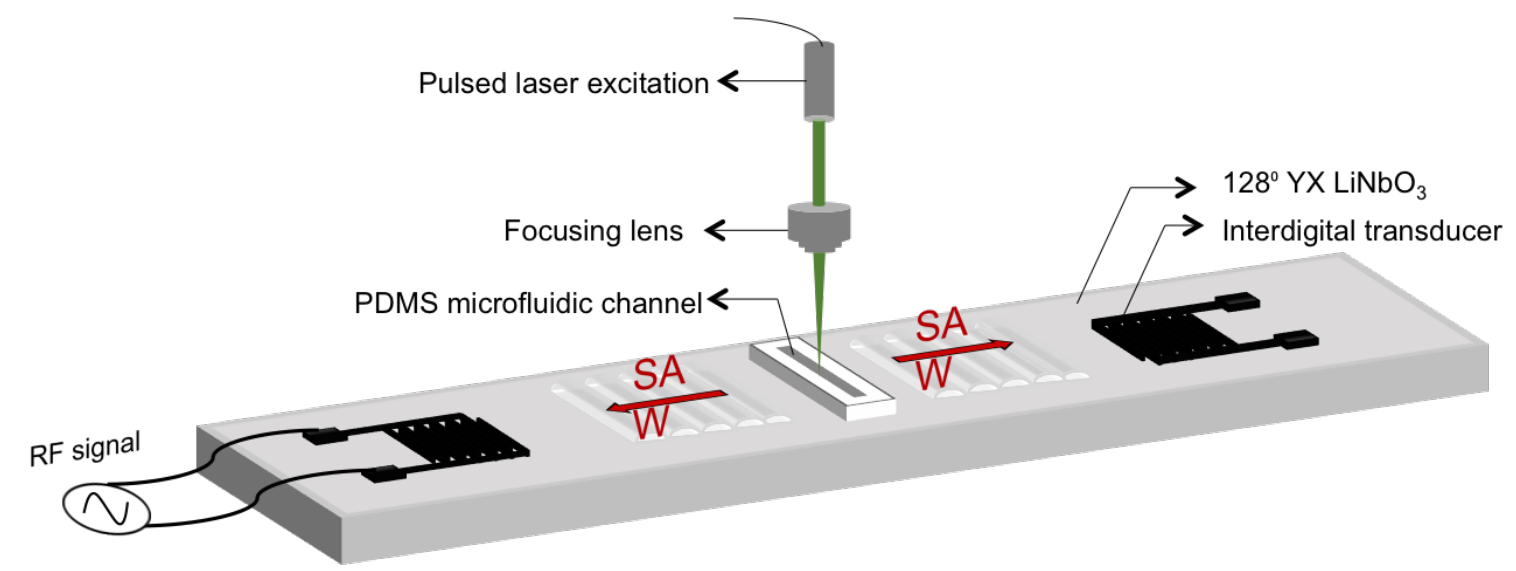

Figure 4.3: Schematic representation of the SAW-PA device. The fluid sample in the microfluidic channel absorbs the pulsed laser and generates longitudinal acoustic waves, which is mode-converted into SAW on the piezoelectric substrate-liquid interface. The SAW is detected using the IDT on the $\mathrm{LiNbO}_{3}$ substrate

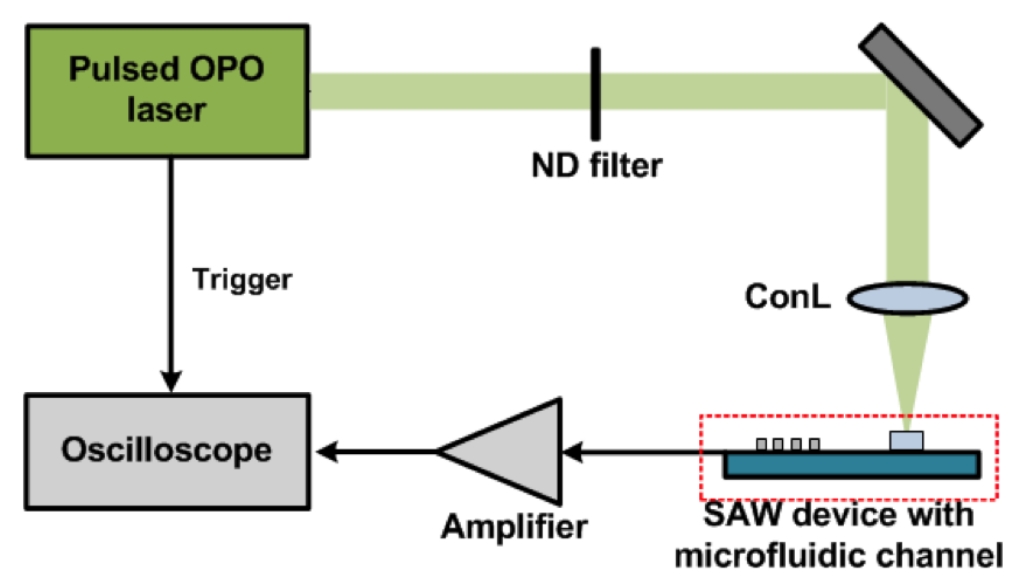

Figure 4.4: The experimental setup for PA measurement using the proposed SAWPA sensor. OPO: optical parametric oscillator; ND: neutral density; ConL: condenser lens; IDT: interdigital transducer 
(a)

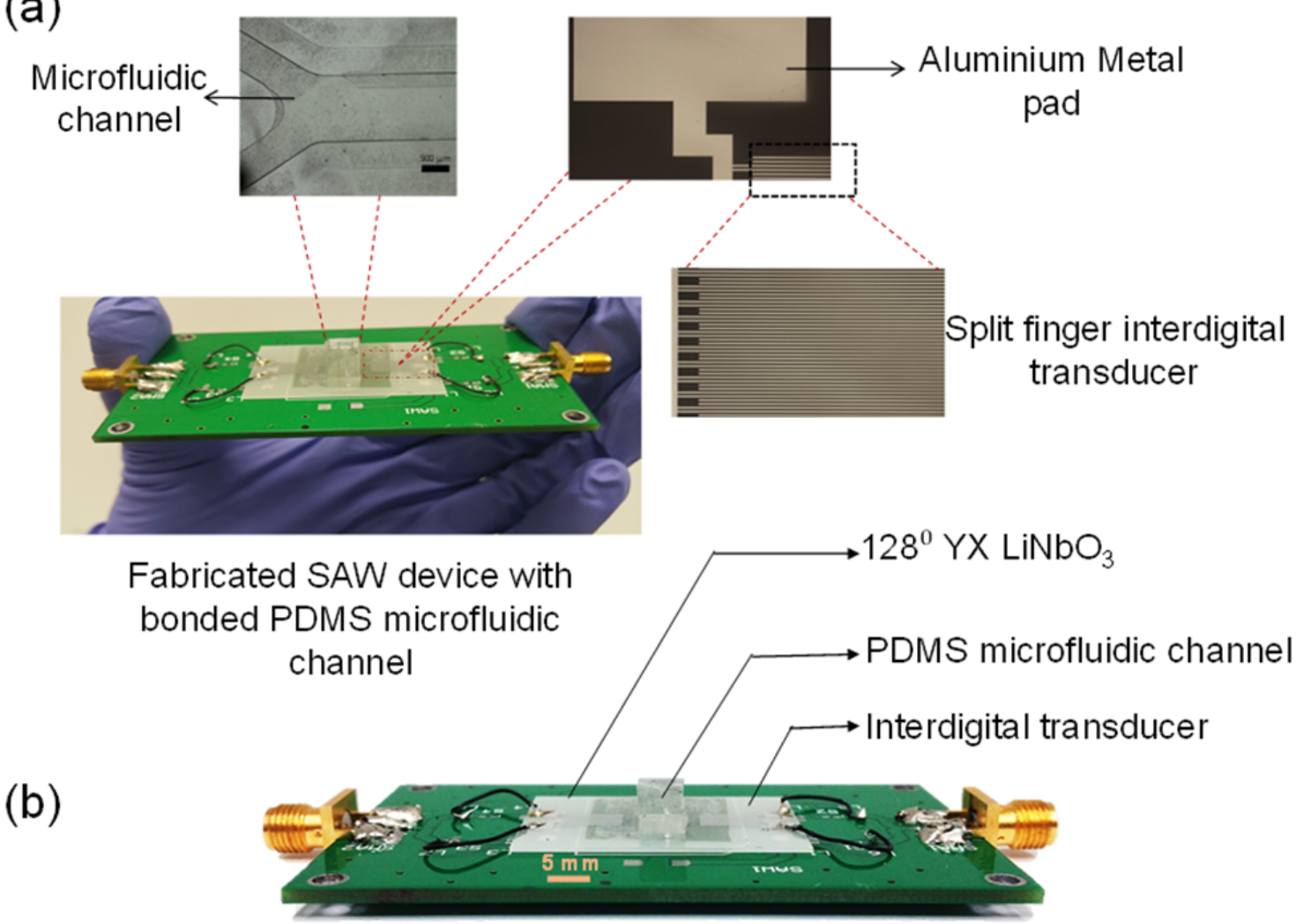

Figure 4.5: The experimental setup for PA measurement using the proposed SAW-PA sensor. (a) Photographs of individual components integrated in the SAW-PA device. (b) Photograph showing side view of SAW-PA sensor with the bonded PDMS microfluidic channel

\subsubsection{Sensor theory and data processing}

\subsubsection{Analytical study of the PA to SAW mode conversion process}

The generated PA is converted to leaky SAW beneath the microchannel, which is then converted into a SAW at the edge of the microchannel. The principle of the mode conversion process involved in exciting a SAW is shown in Fig 4.6. The conversion from leaky wave to surface wave will be 100 percent efficient, if we assume that the substrate fields of the leaky and surface wave are similar [93].The generated PA signal inside the liquid is a longitudinal wave. The conversion process is equivalent to the excitation of the Rayleigh wave by a wedge transducer as mentioned 
in a previous publication [155].A Rayleigh wave is excited on the substrate if it satisfies the condition that $c_{R}$, the Rayleigh wave velocity in the substrate $(3979 \mathrm{~m} / \mathrm{s})[90]$, should be greater than $c_{W}$, the longitudinal wave velocity in the liquid $(1500 \mathrm{~m} / \mathrm{s}$ in water). This condition is satisfied in our configuration. All the longitudinal waves inclined

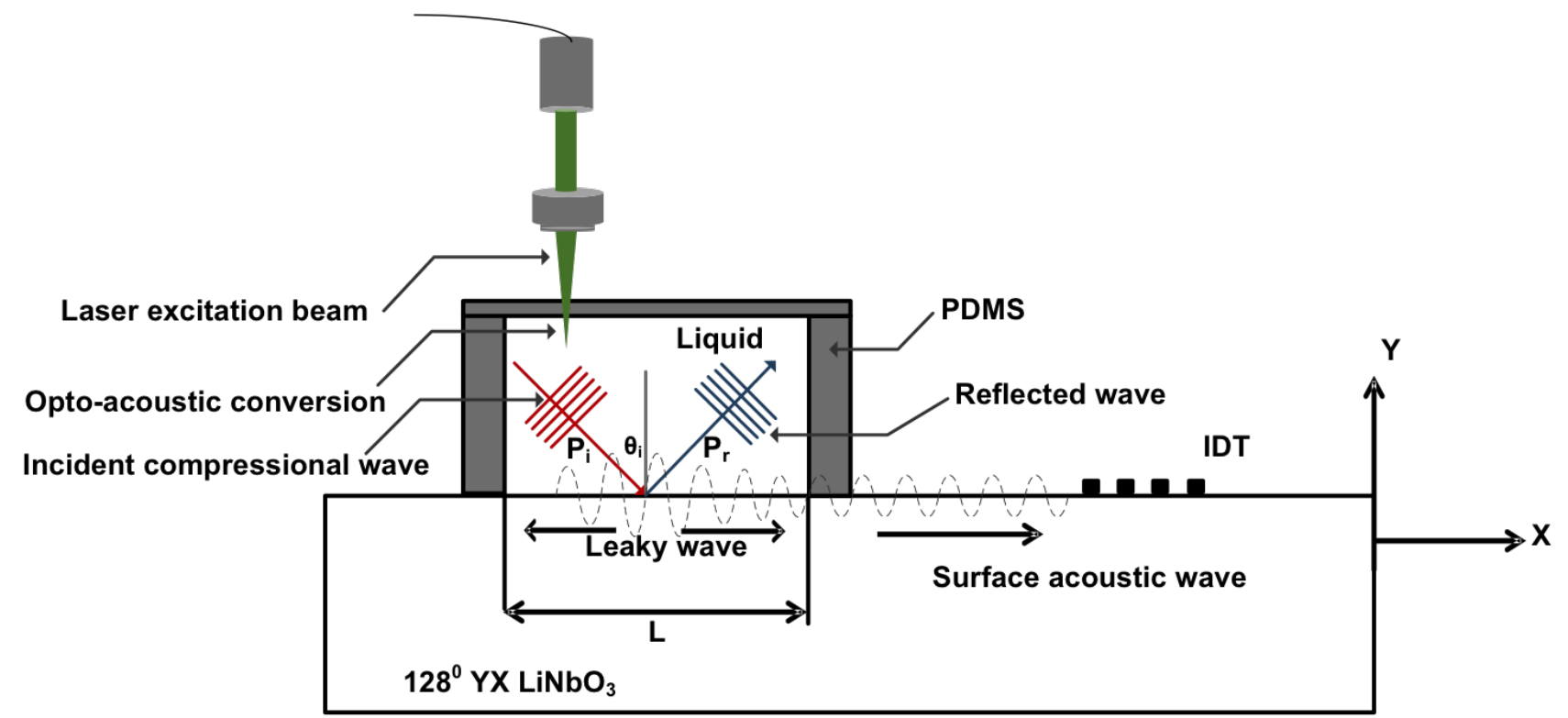

Figure 4.6: Schematic representation of the mode conversion process in generating a Rayleigh surface acoustic wave excitation triggered by the photoacoustic waves. $\mathrm{P}_{\mathrm{i}}$, $\mathrm{P}_{\mathrm{r}}$ and $\theta_{i}$ refer to the incident pressure, reflected pressure and angle of incidence respectively

at an angle $\theta_{i}$ greater than the critical angle defined by: $\sin \theta_{i}=c_{w} / c_{R}$, satisfies the criteria to excite the Rayleigh wave. The efficiency of the conversion process from the longitudinal to the leaky surface wave is calculated analytically by the equation:

$$
\eta_{0}=2\left[1-\exp (-\alpha L]^{2} / \alpha L\right.
$$

where $\alpha$ denotes the leak rate or attenuation per unit length of the surface acoustic wave under the microchannel, which is a function of the frequency of the wave, acoustic impedance in the liquid and the ratio of mode displacement to the power flow. $L$ denotes the aperture or the length on the substrate where the longitudinal wave 
strikes. Fraser et al. [155] plots the efficiency with respect to the factor $\alpha L$ and shows that a conversion efficiency of $81 \%$ is obtained in the mode conversion process. High conversion efficiency of $81 \%$ percent proves the significance of using the SAW as a detector of the longitudinal PA signal.

\subsubsection{Data processing}

The electrical signal received from the SAW device was post-processed to extract the sample generated PA signal from the background. Fig 4.7(a) shows the signal obtained at the IDT due to the laser absorption in the dye for a wavelength of $530 \mathrm{~nm}$. Erythrosine (red) dye was used as the fluidic sample. Laser excitation generates background acoustic signals from the PDMS microfluidic channel. Two control experiments were performed. The first experiment involved detecting the SAW-PA signals produced by the PDMS microchannel (without containing any fluid in it) and the second experiment was conducted with deionized (DI) water as the sample fluid in the microchannel. The transient signal obtained from this control experiment was subtracted from the signal of interest generated by the dye in the microchannel. At $530 \mathrm{~nm}$, the background signal remains relatively weak compared to the signal generated by the dye as shown in Fig 4.7(d). From experiments we observed that the influence of the PDMS was the maximum in the blue spectral region. Fig 4.8 shows the background signal generated by the PDMS microfluidic channel for different optical wavelengths. The power spectral density in Fig 4.7(c) of the time domain signal (0-45 $\mu s$ ) obtained from the dye has a dominant frequency component at $12.9 \mathrm{MHz}$ corresponding to the SAW resonant frequency. Fig 4.9 shows the PSD spectra of the transient signals obtained for the Erythrosine dye from 2-4 $\mu \mathrm{s}$ (red curve) and 6-8 $\mu \mathrm{s}$ (black curve). The spectra show a dominant frequency component at $12.9 \mathrm{MHz}$, which again coincides with the SAW resonant frequency. 

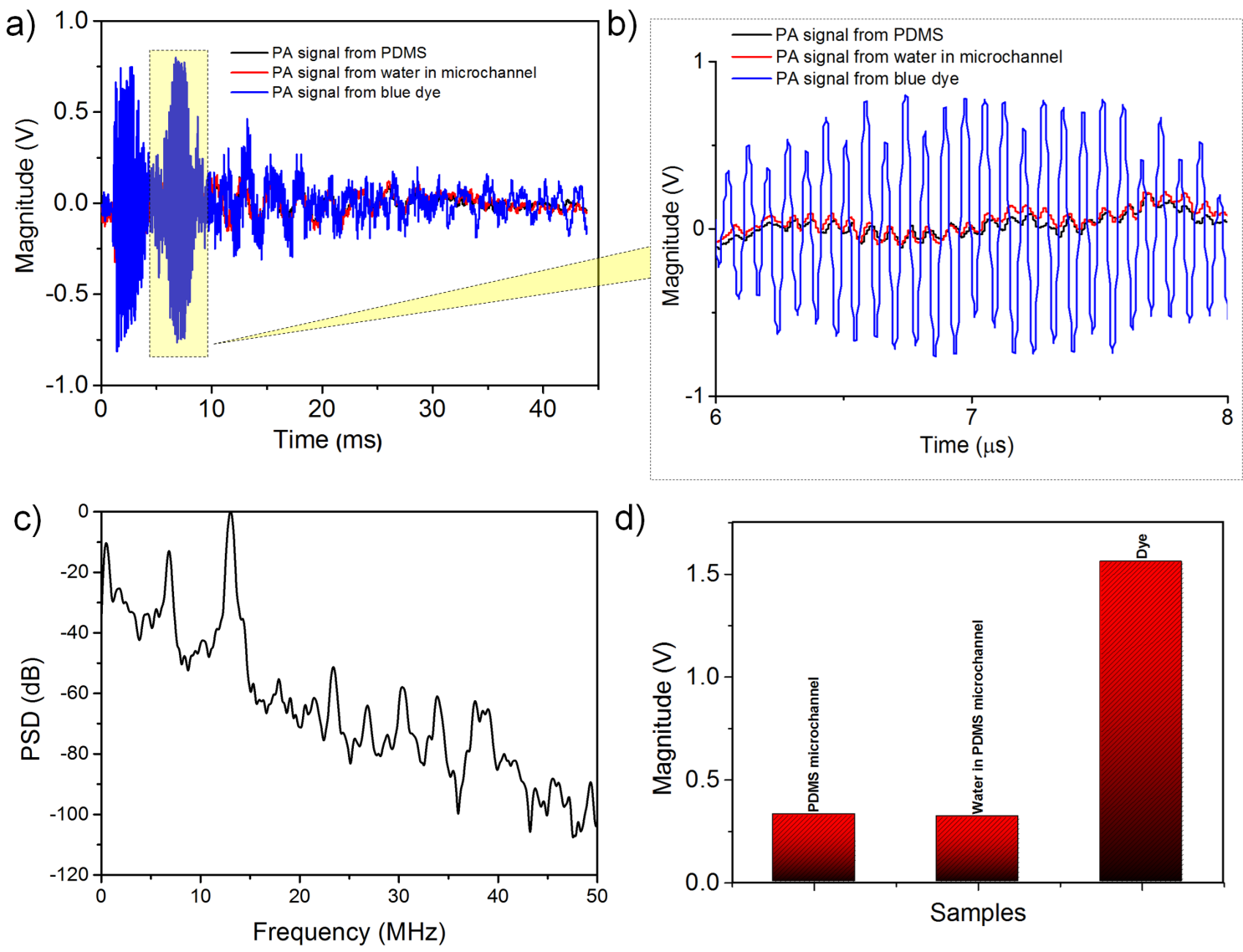

Figure 4.7: Photoacoustic signal output obtained at the IDT: (a) Transient signal generated at the IDT from the absorption of light at wavelength $530 \mathrm{~nm}$ from PDMS microchannel (black curve), DI water (red curve) and Erythrosine dye (blue curve) in the microchannel. (b) Shows the zoomed signal from 6-8 $\mu \mathrm{s}$. Signal from the dye is strong compared to the background signal from PDMS due to the high optical absorption of the dye compared to water and PDMS. (c) Results of the power spectral density (PSD) analysis for entire duration of the transient signal with peak magnitude at the SAW center frequency of $12.9 \mathrm{MHz}$ (d) Signal amplitude for the three different conditions at $530 \mathrm{~nm}$ wavelength.

\subsubsection{Analytical modelling}

The microfluidic channel was approximated as a thin slab (width of $1.15 \mathrm{~mm}$ and thickness of $150 \mu \mathrm{m}$ ). The pressure waveform was approximated as a Heaviside 


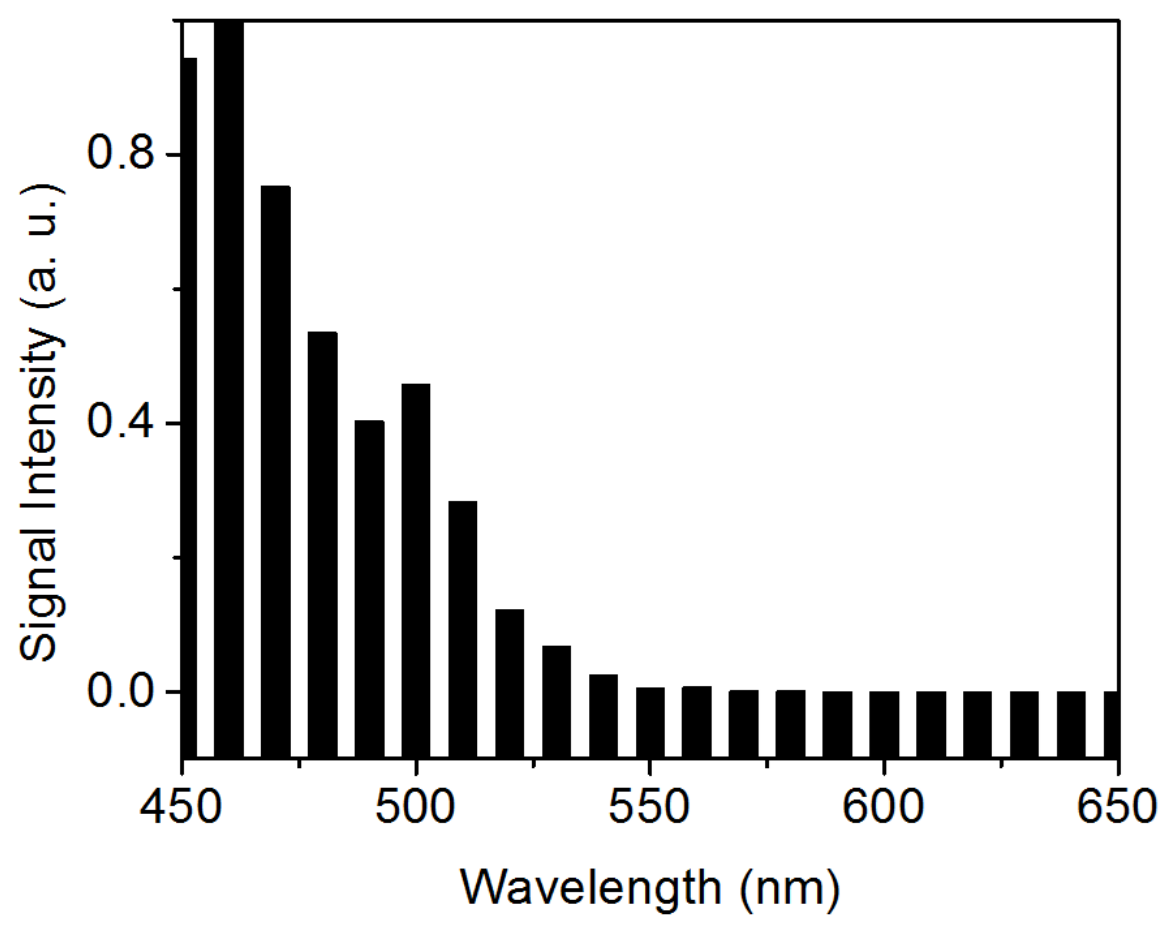

Figure 4.8: Background SAW-PA signal intensity generated by the PDMS microfluidic channel for different optical excitation wavelengths

step function from $-d / 2$ to $+d / 2$ where $d$ denotes the channel thickness. The pressure at the bottom of the channel, which is converted to SAW, is obtained by using the following [87]:

$$
p(z, t)=\frac{1}{2} p_{0}\left(z-v_{s t}\right)+\frac{1}{2} p_{0}\left(z+v_{s t}\right)
$$

The acoustic pressure at $z(=-d / 2)$ is a rectangular pulse of width $d / v_{s}$, with a thickness (d) c. a. $150 \mu \mathrm{m}$ and acoustic velocity $\left(v_{s}\right)$ of $1500 \mathrm{~m} / \mathrm{s}$ in water generates a pulse of width $0.1 \mu \mathrm{s}$. The corresponding frequency spectrum is a sinc function with the first minima at $10 \mathrm{MHz}$. Deconvolution of the SAW-PA signal from the SAW transfer function results in a low pass signal with a $-40 \mathrm{~dB}$ response at $10 \mathrm{MHz}$ which represents the main lobe of the expected PA frequency spectrum. Hence, the generated PA spectrum agrees with the theoretical analysis. The repeatability of the signal was confirmed 


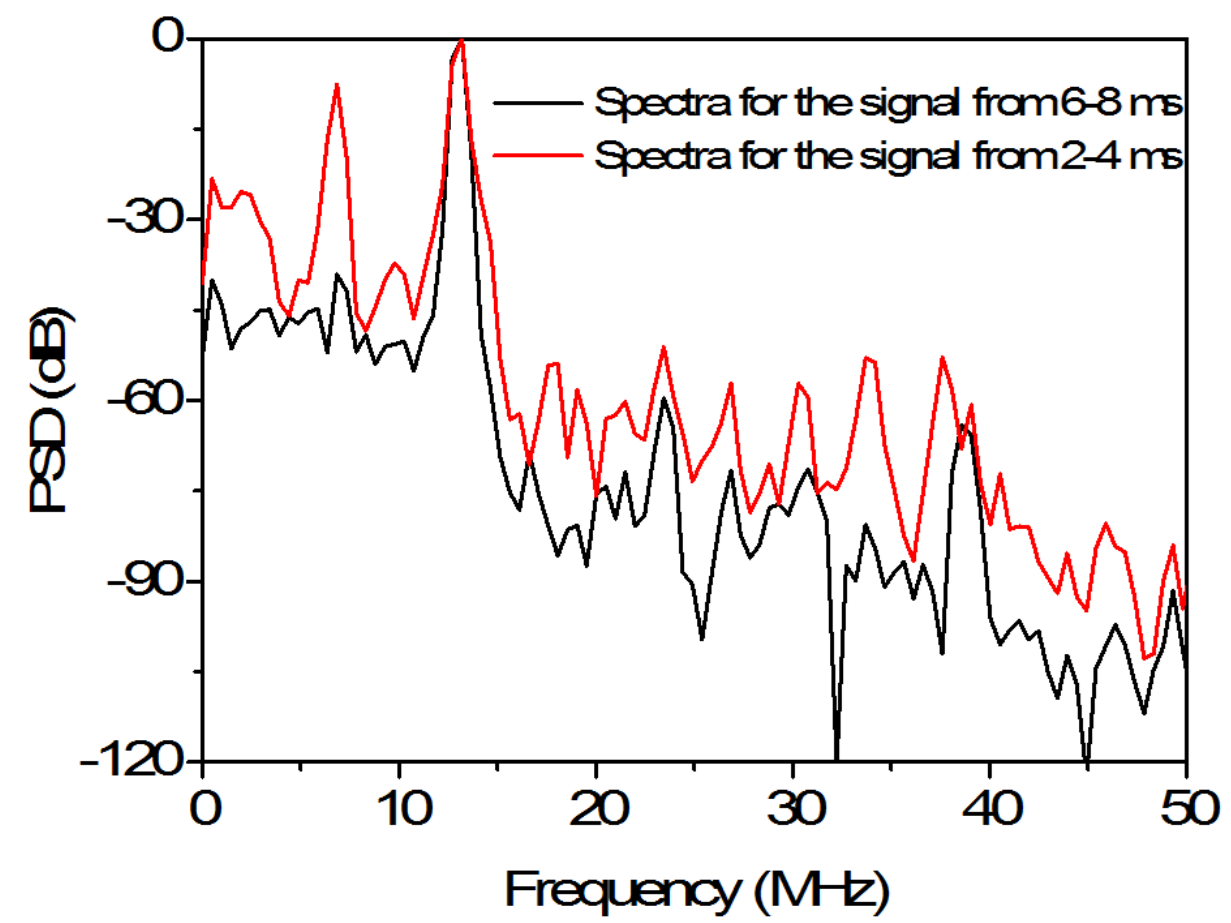

Figure 4.9: Results of the power spectral density (PSD) analysis for specific durations (2-4 $\mu s$ and 6-8 $\mu s)$ of the transient signal displaying the dominant frequency component at $12.9 \mathrm{MHz}$

by using Pearsons linear correlation coefficients on multiple sets of transient data as shown in Fig 4.10. To illustrate the reproducibility of the transient data shown in Figure 4.7(a), we take a set of time domain data obtained for erythrosine dye solutions of various concentrations ( 75,50 and $25 \%$ volume concentrations each) and also calculated the cross-correlation between the transient signals to highlight the robustness of the signal. The repeatability can be estimated by correlation analysis [156].The Pearsons linear correlation coefficients for the transient signals obtained for the signals $\mathrm{s} 1$ and s2 in Figure 4.10 is 0.816 , and for signals s2 and s3 is 0.904 . A value greater than +0.7 indicates very high correlation between signals. Hence, the transient data is repeatable 
(a)

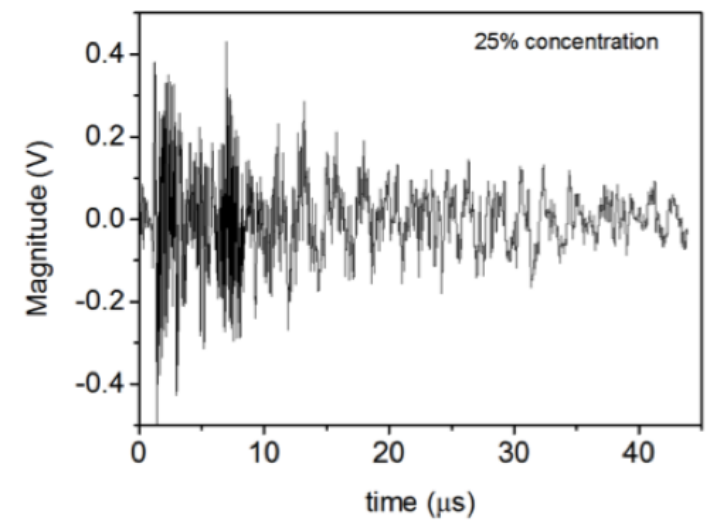

(b)

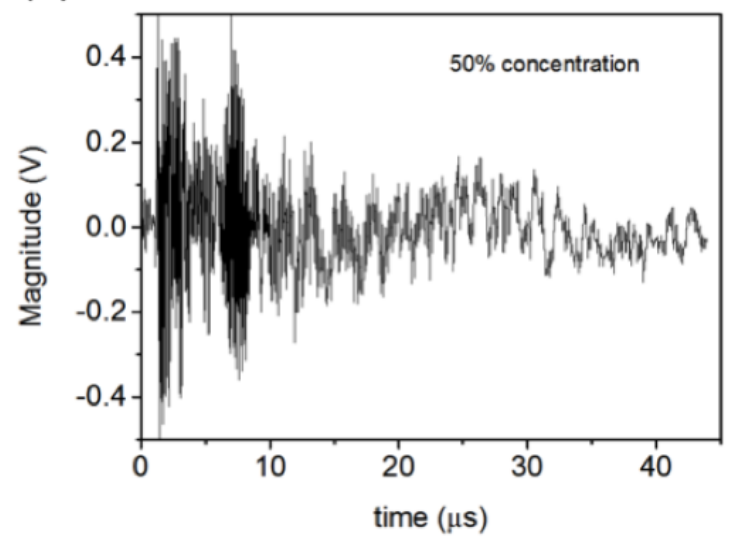

(c)

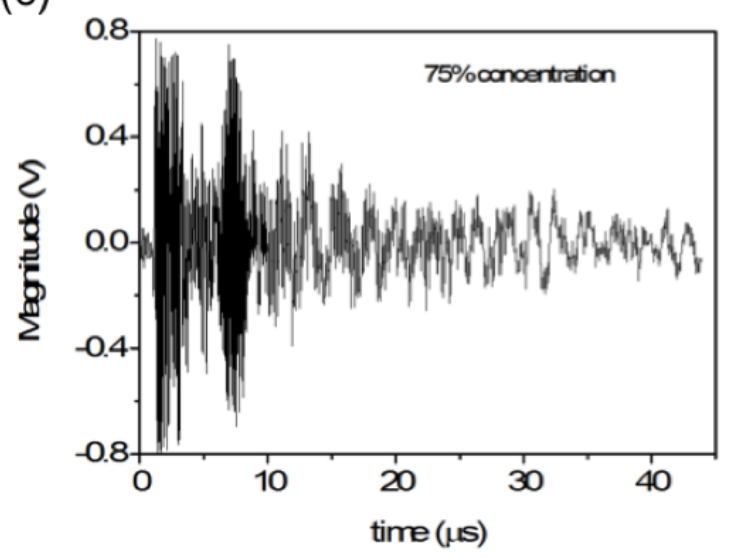

Figure 4.10: Repeatability of the data: Transient signals obtained for various Erythrosine dye concentrations: a) $25 \%$, b) $50 \%$ and c) $75 \%$.

\subsection{Experimental Results and discussion}

\subsubsection{SAW-PA signal from standard dye solutions}

\subsubsection{Dye absorption spectropscy using SAW-PA device}

The UV/Vis absorption spectroscopy measurement was performed on the erythrosine (red) and the brilliant blue dyes prior to performing the SAW-PA device characterization. UV/Vis absorption spectra presented in Fig 4.11(a) for the erythrosine $\left(6 \times 10^{-6} \mathrm{M}\right)$ and brilliant blue $\left(6 \times 10^{-6} \mathrm{M}\right)$ dyes show characteristic absorption max- 
imums at $510 \mathrm{~nm}$ and $620 \mathrm{~nm}$ respectively. During our first set of experiments with the SAW-PA device, the dyes were introduced into the microchannel and the entire device was kept in a shock-free assembly during the measurement. The pulsed-laser repetition rate was set to a low value of $1 \mathrm{~Hz}$ to prevent increase in temperature and subsequent heating of sample in the microchannel. The acoustic signal obtained was further post-processed using MATLAB (Matrix Laboratory Programming Language). A band-pass filter with the center frequency of $12.9 \mathrm{MHz}$ and a 6-dB bandwidth of $4 \mathrm{MHz}$ filters the other signal frequencies. The maximum peak-peak amplitude for each optical wavelength excitation was subtracted from the background signal amplitude obtained for the same wavelength. The signal was further normalised against the laser intensities. In Fig 4.11(b) the red symbols shows the signal obtained from erythrosine loaded in the SAW-PA device for laser wavelength excitations varying in the $450 \mathrm{~nm}$ to $650 \mathrm{~nm}$ spectral range. The measurements were repeated three times
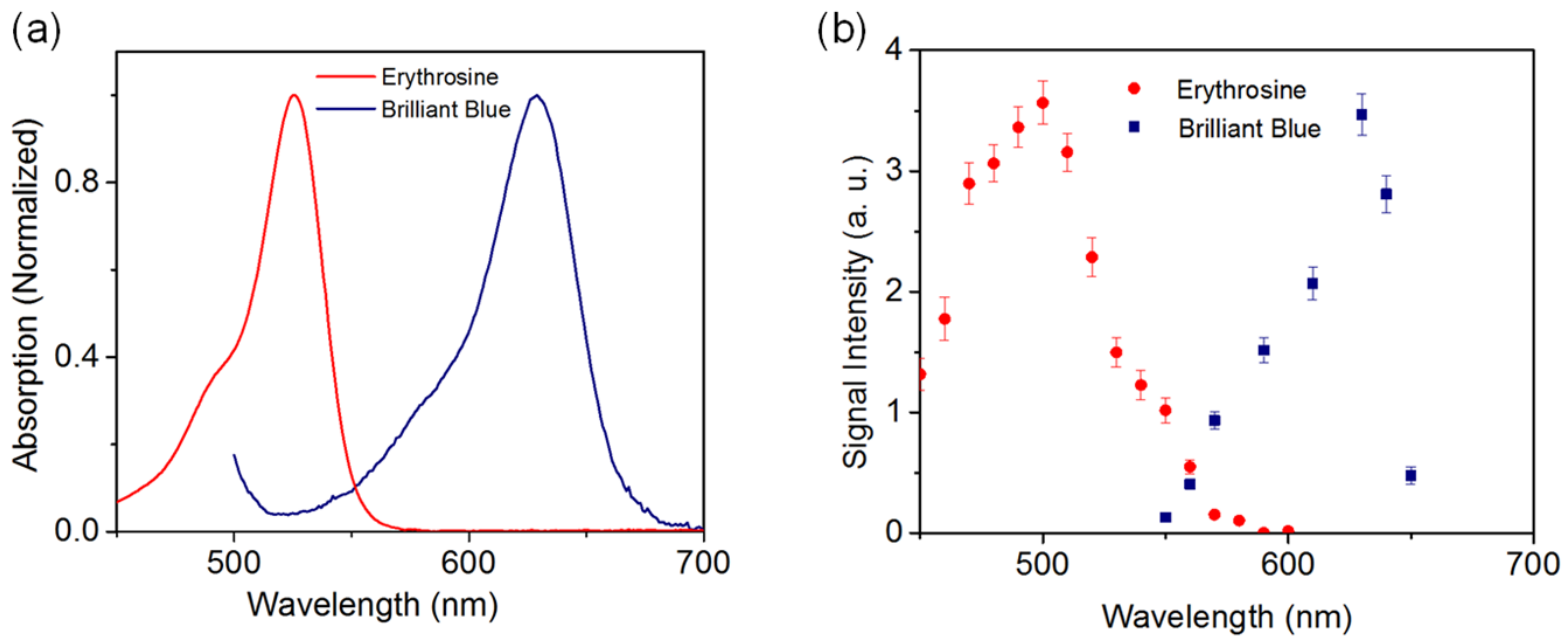

Figure 4.11: (a) UV/Vis absorption spectrum of erythrosine and brilliant blue (b) Measured SAW-PA signal intensity from the erythrosine and brilliant blue dyes at different laser wavelengths.

to confirm repeatability and the variation was less than $10 \%$. Measured peaks for the erythrosine and the brilliant blue dye were observed at $500 \mathrm{~nm}$ and $620 \mathrm{~nm}$ respec- 
tively, which was in good agreement with the absorption maxima of the dye found in Fig 4.11(a). It must be also noted that a $10 \mathrm{~nm}$ blue shift for the mean value of the measured red dye spectra against the expected peak could be attributed to variations in the laser intensity and laser focusing at different wavelengths.

\subsubsection{Effect of concentration on the SAW-PA signal}

Further experiments were conducted using the SAW-PA device to evaluate the PA signal generated on the SAW device for different concentrations of the dye. Erythrosine dye was used as the sample liquid. The dye was diluted with DI water at room temperature $\left(23^{\circ} \mathrm{C}\right)$ to prepare various volume concentrations ranging from 0 to $100 \%$. The laser light at a fixed wavelength of $530 \mathrm{~nm}$ with pulsed excitation was used to illuminate the sample. Fig 4.12 shows the magnitude of the signal received by the SAW-PA device for different concentrations of the erythrosine dye. The amplitude of the PA signal depends on the optical absorption coefficient $\left(\mu_{a}\right)$ and the path length (I). For $\mu_{a} L \ll 1$, the PA signal is directly proportional to the optical absorption coefficient and hence the SAW-PA signal increases with concentrations. However, at higher concentrations the optical penetration depth becomes smaller than the path length, causing saturation of the signal. A previous research [157] has reported the PA amplitude of the form mentioned in Eq. 4.3 for variation in the optical absorption coefficient, which is proportional to the concentration.

$$
P A\left(z, \mu_{a}\right)=\mu_{a} F_{0} K_{1} \exp \left(-\mu_{a} z\right)
$$

where $\mu_{a}$ is the optical absorption coefficient of the medium which is the product of concentration and molar extinction coefficient, $F_{0}$ is the optical energy received per unit area; $k_{1}$ is the PA conversion factor, and $z$ denotes the depth of the microchannel. An exponential fitting function of the form mentioned in Eq. 4.4 is used to correlate with 


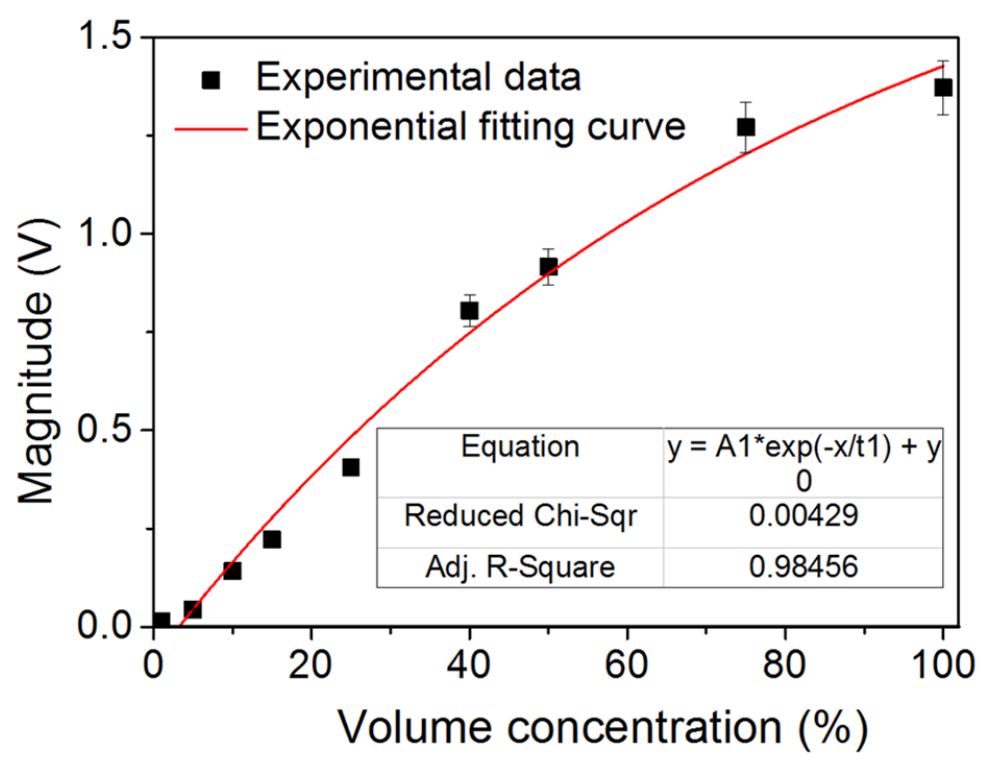

Figure 4.12: Signal received by the SAW-PA device for different concentration of the dye (expressed in volume percentage). An exponential function of the form mentioned in Eq. 4.4 was used to fit the experimental data. Error bars are presented to confirm the repeatability of the photoacoustic signal received on the SAW-PA device. The model equation fits to the experimental results with an R-square value of 0.985 .

Eq. 4.3 and incorporate the saturation of the PA signal.

$$
Y=Y_{0}+A_{1} \exp \left(-x / t_{1}\right)
$$

where $Y_{0}, A_{1}$ and $t_{1}$ are the unknown parameters. $x$ denotes the concentration of the solution. $Y_{0}$ indicates the saturation voltage level due to increasing concentration. $A_{1} \exp \left(-x / t_{1}\right) / t_{1}$ depicts the sensitivity and $t_{1}$ denote the concentration at which the signal saturates. After fitting the model to the experimental data, the obtained values for the unknowns are $Y_{0}=2.04, A_{1}=-2.123$ and $t_{1}=80.625$. The goodness of fit has an R-square value of 0.985 . The SAW-PA amplitude reaches close to saturation $\left(\mathrm{e}^{-1}\right)$ at a concentration of $80.625 \%$. The dye with various concentrations was analysed five times to confirm the repeatability. Each reading was averaged over 200 sweeps to improve the signal-noise ratio. As shown in Fig 4.12, a maximum standard deviation c.a $4.8 \%$ for $75 \%$ volume concentration was obtained, which indicates good repeatability 
in the current SAW-PA device.

\subsubsection{Effect of viscosity on the SAW-PA signal}

The ability of our platform to detect the mechanical parameters of a liquid sample was confirmed by sensing its viscosity. For this study, erythrosine dye-glycerol (EG) solutions of varying concentrations were prepared at room temperature $\left(23^{\circ} \mathrm{C}\right)$ and used as samples for the viscosity tests. The effect of viscosity was analysed by comparing the received SAW-PA signal from the EG solution with the erythrosine dyewater $(\mathrm{EW})$ solution of similar dye concentrations. The laser light at a fixed wavelength of $530 \mathrm{~nm}$ with pulsed excitation was used to illuminate the sample. Glycerol added to the dye induces optical clearing effects to reduce scattering and therefore enhanced the SAW-PA signal [158]. Fig 4.13 shows the percentage increase in the SAW-PA signal amplitude of the EG solution with respect to the EW solution for various dye concentrations. At 50\% dye concentration, a $65 \%$ increase in the signal intensity was observed for the EG solution. This result demonstrates a clear enhancement in the SAW-PA signal amplitudes due to increasing viscosity of the fluids. At higher dye concentrations, the viscosity of the EG solution decreases and becomes comparable to the EW solution, which reduces the relative magnitude of the SAW-PA signal intensity.

\subsubsection{SAW-PA signals from gold nanoparticles}

Metal nanoparticles (NPs) with high plasmon absorption in the visible and the near-infrared wavelengths provide enhanced capabilities for the specific detection of analytes for biosensing applications. The ability of the SAW-PA device to detect plasmonic absorption of gold nanoparticles (GNPs) and gold nanorods (GNRs) was investigated by introducing GNPs and GNRs (stabilised with CTAB surfactant) dispersed in water into the microchannel. The plasmonic absorption and subsequent 


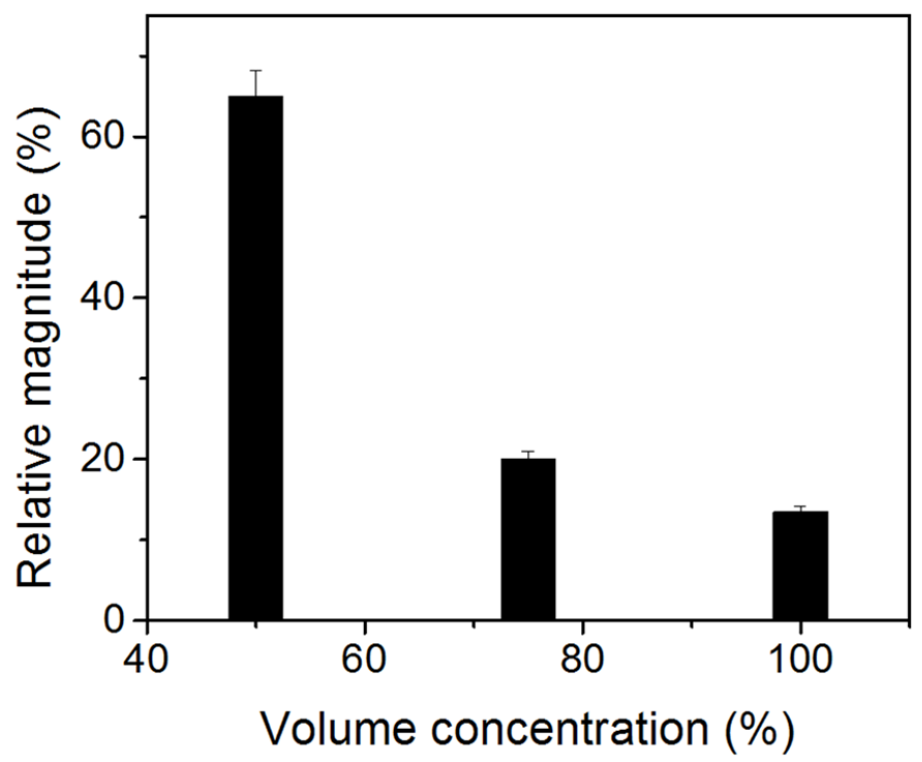

Figure 4.13: Percentage increase in the SAW-PA signal intensity of the erythrosine dye-glycerol with respect to the erythrosine dye-water solutions for varying dye concentrations

nonradiative relaxation dominated PA generation for optically heterogeneous nanostructures differ fundamentally from the PA signals generated by the optically homogenous dye solutions.A pulsed laser with wavelengths $480-600 \mathrm{~nm}$ was used for spectroscopic characterization of the nanoparticles. The signal received from the SAW-PA device was averaged over 300 sweeps. Water in the PDMS microchannel was used as the reference solution to which the GNP induced SAW-PA signal was compared. Fig 4.14(a) shows the transient SAW-PA signal from $12 \mathrm{~nm}$ GNP solution (2 $\mathrm{mg} \mathrm{mL}^{-1}$ ) compared to water as a reference, at an excitation wavelength of $520 \mathrm{~nm}$. As shown in Fig 4.14(a), an apparent enhancement in the SAW-PA signal was observed for the GNPs in the microchannel. We further measured the spectroscopic response of the GNPs. Fig 4.14(b) shows the UV/Vis absorption spectrum of GNPs $\left(2 \mathrm{mg} \mathrm{mL}^{-1}\right.$, c. a. $6 \times 10^{12}$ particles $\mathrm{mL}^{-1}$ ) in aqueous solution, with an absorption maximum at $520 \mathrm{~nm}$. The SAW-PA signal (normalised to laser intensity) shown in Fig 4.14(c) follows the absorption profile from $500 \mathrm{~nm}$ and higher wavelengths, with an absorption peak at $520 \mathrm{~nm}$. The deviations at lower wavelengths (less than $500 \mathrm{~nm}$ ) were also reported 
in a previous work, which was attributed to scattering effects [159].

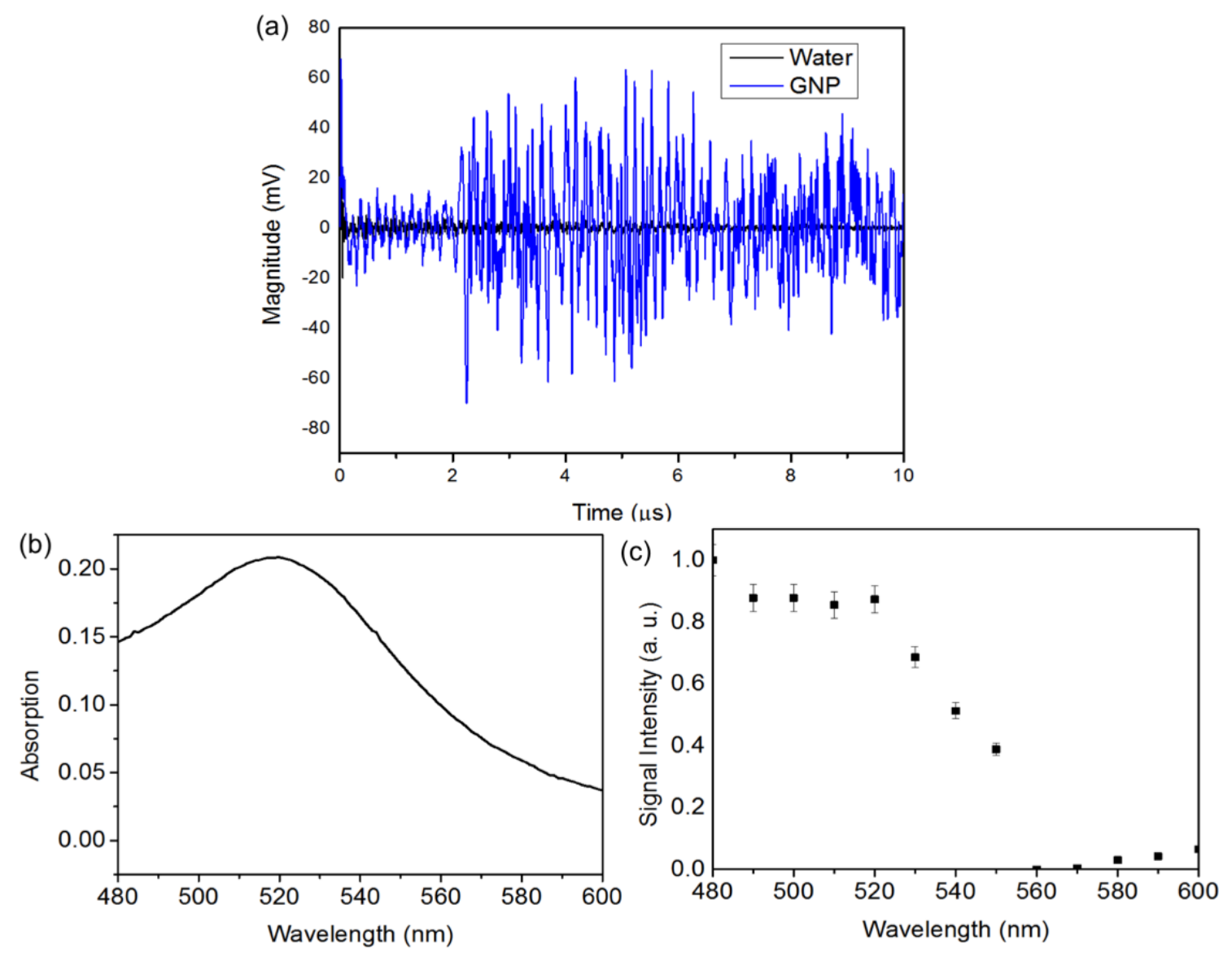

Figure 4.14: (a) SAW-PA signals from $12 \mathrm{~nm}$ GNPs and water in the microfluidic channel for an excitation wavelength of $520 \mathrm{~nm}$. (b) UV/Vis absorption of GNP $\left(2 \mathrm{mg} \mathrm{mL}^{-1}\right)$ (c) SAW-PA signal intensity of GNPs $\left(2 \mathrm{mg} \mathrm{mL}^{-1}\right)$ for different laser wavelengths

\subsubsection{Influence of gold nanoparticle sizes}

Spherical GNPs of various sizes (10 nm-50 nm in diameter) were diluted in water to obtain identical extinction values $\left(0.7 \mathrm{~cm}^{-1}\right)$ at an excitation wavelength of 532 nm. Fig 4.15 shows that the SAW-PA signal for different sizes of the GNPs at an excitation wavelength of $532 \mathrm{~nm}$ increases as a third-order polynomial function of the NP diameter. The standard deviation was calculated from five repeated measurements. The NPs generate nonlinear PA signals at a threshold fluence of $300 \mathrm{~mJ} \mathrm{~cm}^{-2}$ [160]. 
Beyond the threshold fluence, the temperature of the NPs quickly increases to the boiling point of the surrounding water and generates nanobubbles. Previous researchers have proposed analytical models for the bubble generation and dependence of the NP diameter on PA signal amplitude. Accordingly, the PA signal amplitude increases with NP sizes [161]. The threshold for the nonlinearity in the dye is higher than the nanoparticle. For the Erythrosine dye with an optical absorption coefficient of 100 $\mathrm{cm}^{-1}$, the temperature rise is c.a $0.5^{\circ} \mathrm{C}$. Hence, the nonlinear behaviour in dye is less profound than in the NPs.

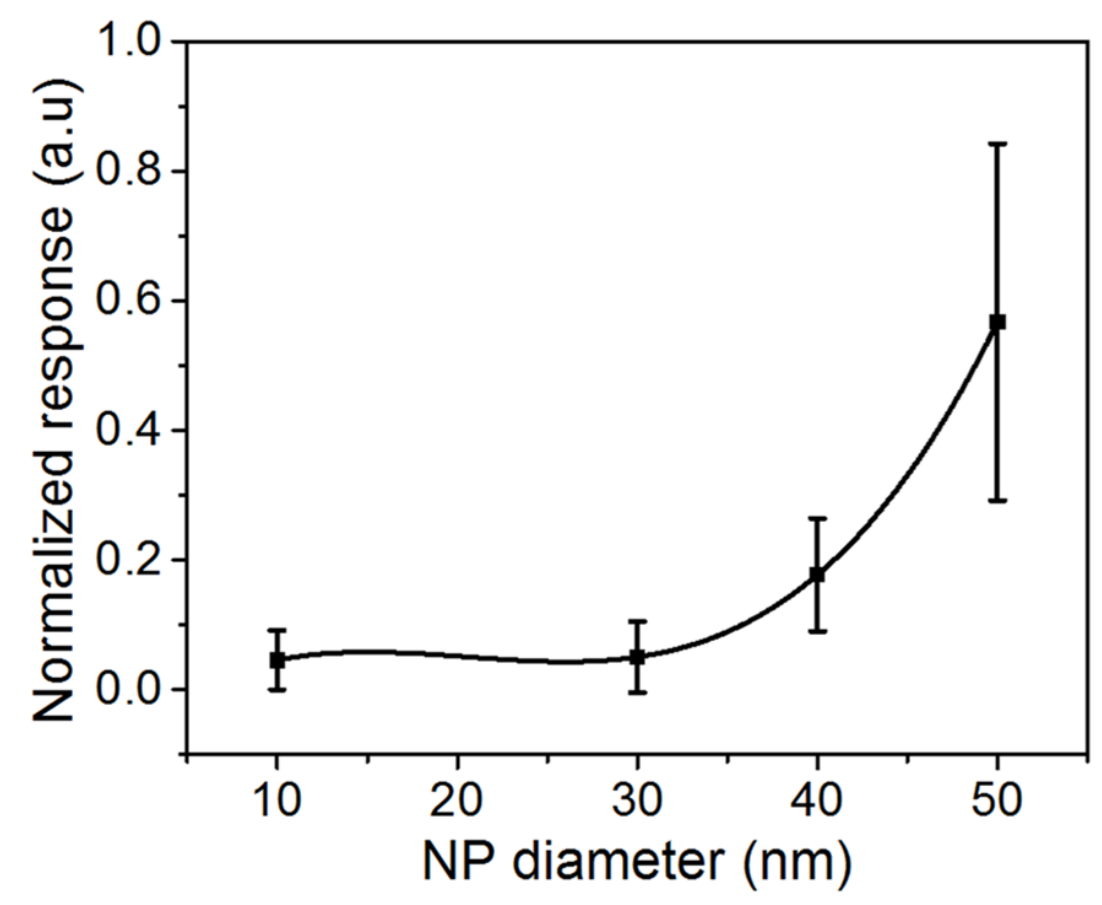

Figure 4.15: Normalized SAW-PA response for different GNP sizes at optical wavelength of $532 \mathrm{~nm}$. The fitting curve represents a third order polynomial function.

\subsubsection{Sensing the transverse and longitudinal plasmon modes of gold nanorods}

The efficacy of the SAW-PA for biosensing applications can be further emphasised by sensing the plasmon resonance modes of the gold nanorods (GNRs) at visible and near-infrared regions. The longitudinal plasmon bands are extremely sen- 
sitive to changes in the dielectric properties of the surrounding medium, improving the detection signal-noise ratio [162]. GNR of dimension $10 \mathrm{~nm} \times 85 \mathrm{~nm}$, with the transverse and longitudinal plasmon resonance modes at $512 \mathrm{~nm}$ and $1064 \mathrm{~nm}$ respectively were used for the study. Fig 4.16 illustrates the normalized SAW-PA response from

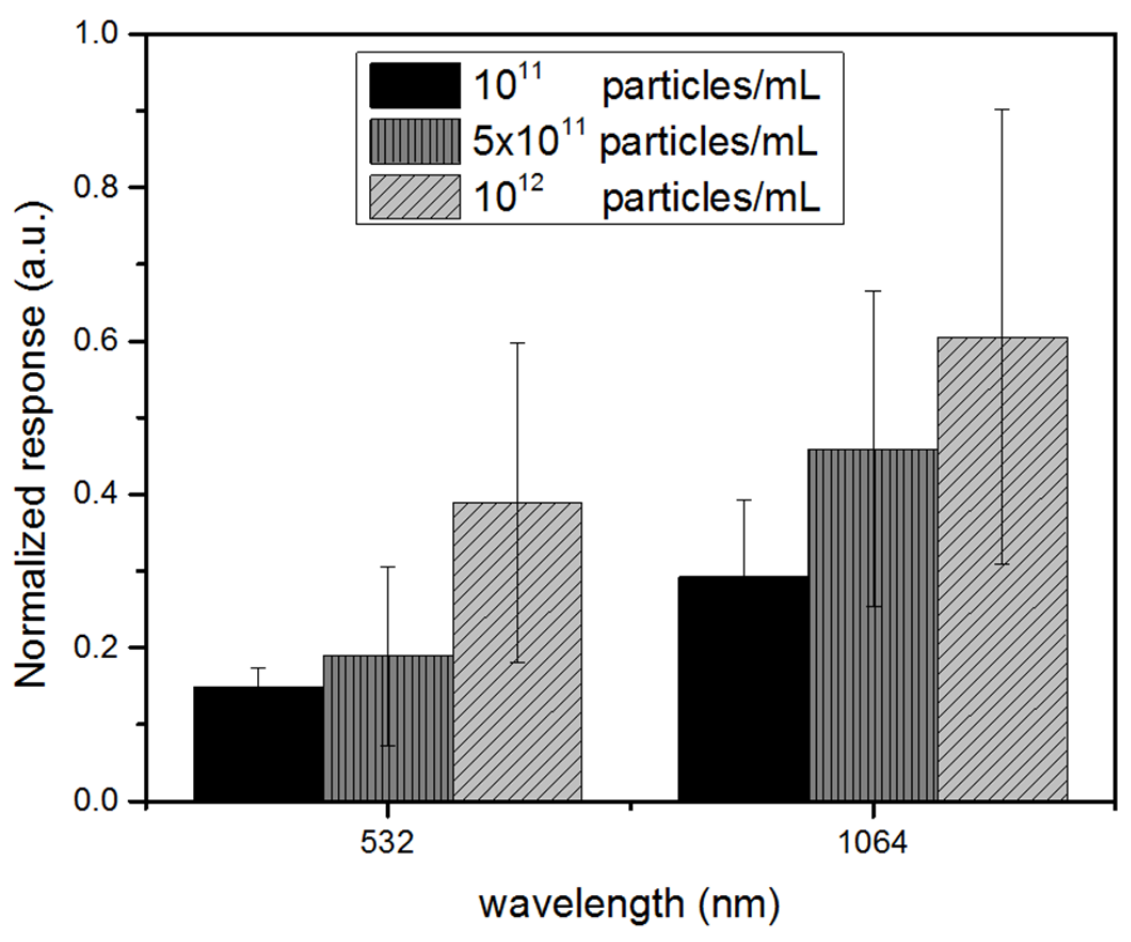

Figure 4.16: SAW-PA response of the gold nanorods $(10 \mathrm{~nm} \times 85 \mathrm{~nm})$ at $532 \mathrm{~nm}$ and $1064 \mathrm{~nm}$ for different concentrations.

various concentrations of GNR solutions at optical excitation wavelengths of $532 \mathrm{~nm}$ and $1064 \mathrm{~nm}$. At each concentration, mean and standard deviations were obtained with five repeated measurements. The significant increase in the SAW-PA signal at $1064 \mathrm{~nm}$ compared to the amplitude at $532 \mathrm{~nm}$ conforms to the longitudinal resonance of the GNRs. 


\subsubsection{Influence of shape of nanoparticles}

The SAW-PA response of the GNP and GNR was compared by selecting three different nanoparticle solutions at a concentration of $2 \mathrm{mg} \mathrm{mL}^{-1}$. The samples consisted of $40 \mathrm{~nm}$ (diameter) gold nanosphere, GNRs of dimension $10 \mathrm{~nm} \times 85 \mathrm{~nm}$ (GNR1) and $10 \mathrm{~nm} \times 40 \mathrm{~nm}$ (GNR2) -diameter $\times$ length. Fig 4.17 shows the TEM images of the nanoparticles with corresponding UV-Vis absorption spectra. The GNP

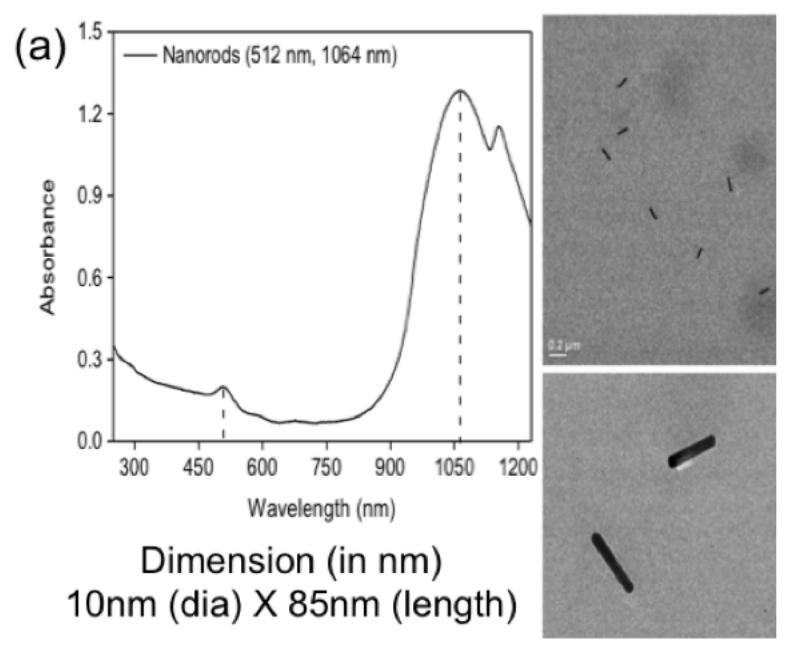

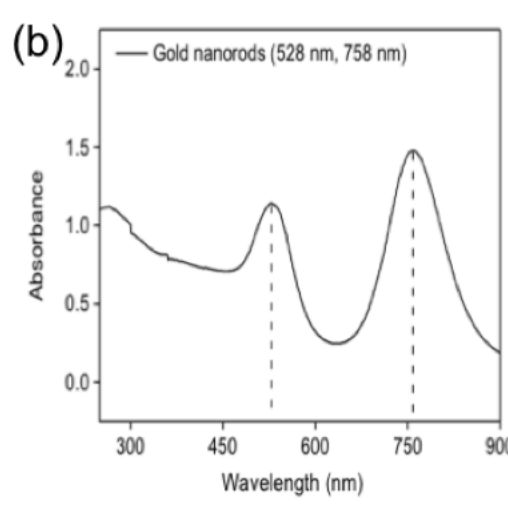

Dimension (in $\mathrm{nm}$ )

$10 \mathrm{~nm}$ (dia) X 40nm (length)
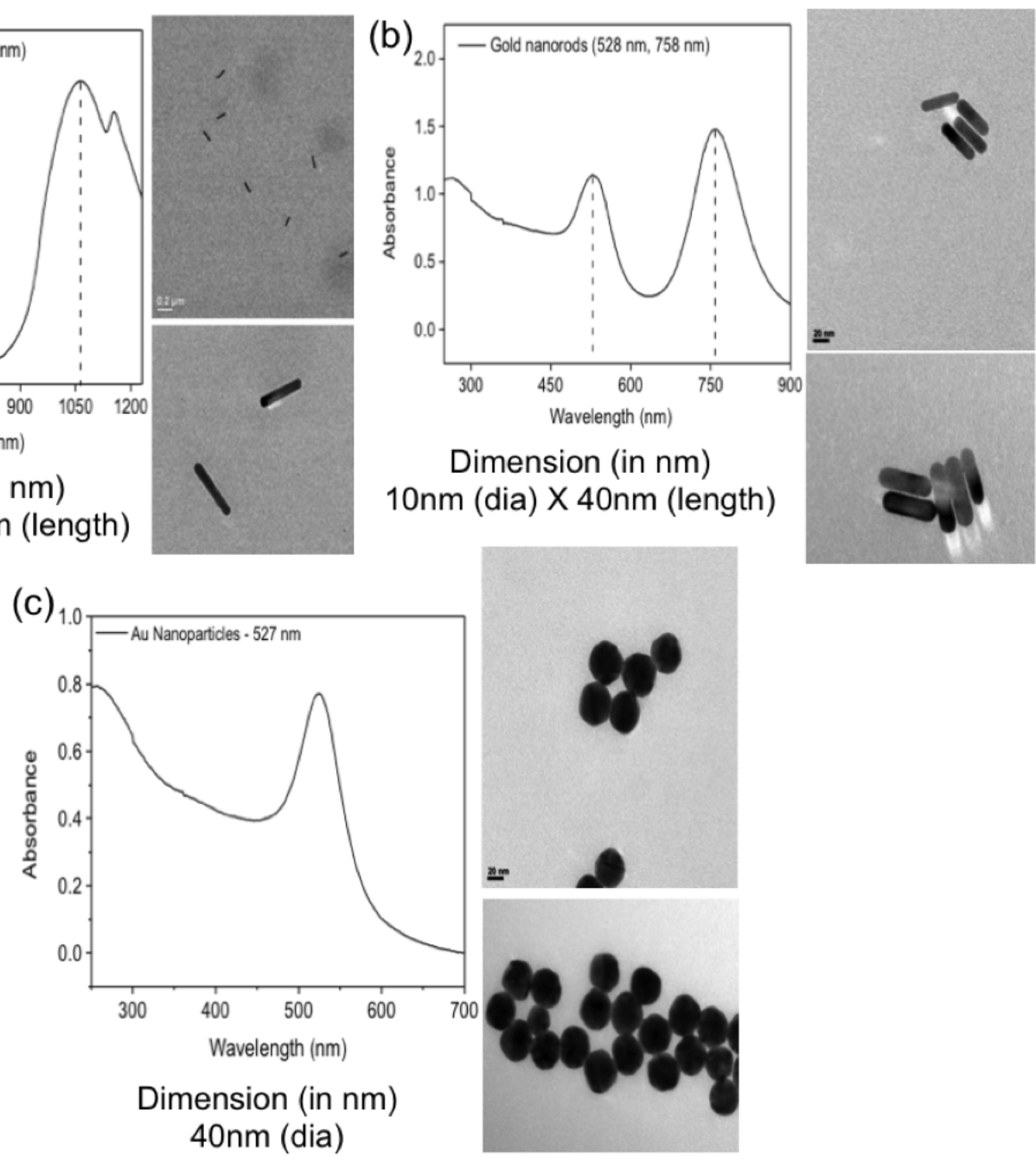

Figure 4.17: UV-vis absorption spectra and TEM images of the gold nanostructures. (a),(b) and (c) represents the UV-vis absorption spectrum of the $10 \mathrm{~nm} \times 85 \mathrm{~nm}$ gold nanorod, $10 \mathrm{~nm} \times 40 \mathrm{~nm}$ gold nanorod and $40 \mathrm{~nm}$ gold nanoparticle respectively with the TEM images of the corresponding nanostructures. 
has a peak plasmon resonance at $527 \mathrm{~nm}$. The two GNR's selected has a transverse plasmon absorption peak at $512 \mathrm{~nm}$ and $528 \mathrm{~nm}$ and corresponding longitudinal peaks at $1064 \mathrm{~nm}$ and $758 \mathrm{~nm}$. Fig 4.18 shows the experimental results for the SAW-PA response for the three nanoparticle solutions. From the graphs, at $800 \mathrm{~nm}$ and 1064 $\mathrm{nm}$ optical wavelengths, the GNR2 and GNR1 have the maximum signal amplitudes respectively. GNR2 with an effective radius of 21.5 and aspect ratio of 3.4 has a per micron optical absorption at $800 \mathrm{~nm}$ of ca. $400 \mu^{-1}$ and the optical absorption of GNP at $532 \mathrm{~nm}$ is $75 \mu^{-1}$ (ca.) [163]. This explains the reason for larger signal amplitude at $800 \mathrm{~nm}$ for GNR2 compared to GNP at $532 \mathrm{~nm}$. GNRs with high aspect ratio and smaller effective radius increase the optical absorption leading to improved SAW-PA response. The lower response for the GNR1, which has an effective radius of $25 \mathrm{~nm}$ and an aspect ratio of 5.48 at $1064 \mathrm{~nm}$, is attributed to the increased contribution of scattering to the absorption, which increases nonlinearly with effective radius. The variation in morphology of the nanoparticle shifts the peak absorption wavelength, which is determined by measuring the optical wavelength at which the SAW-PA response is the maximum as shown in Fig 4.18.

\subsection{FEM modelling of the SAW-PA sensor}

\subsubsection{Theoretical analysis}

According to PA theory, a laser pulse duration shorter than the acoustic and thermal confinement time is considered as a source of delta heating [87]. The corresponding PA has a wideband spectrum. Strohm et al. [164] performed FEM studies for determining PA frequency response from micron sized particles with different boundary conditions. In the SAW-PA sensor, the PA source is surrounded by the PDMS microfluidic channel and piezoelectric substrate. The reflections from the boundary modify the PA frequency spectrum. The PA generated inside the microfluidic channel 


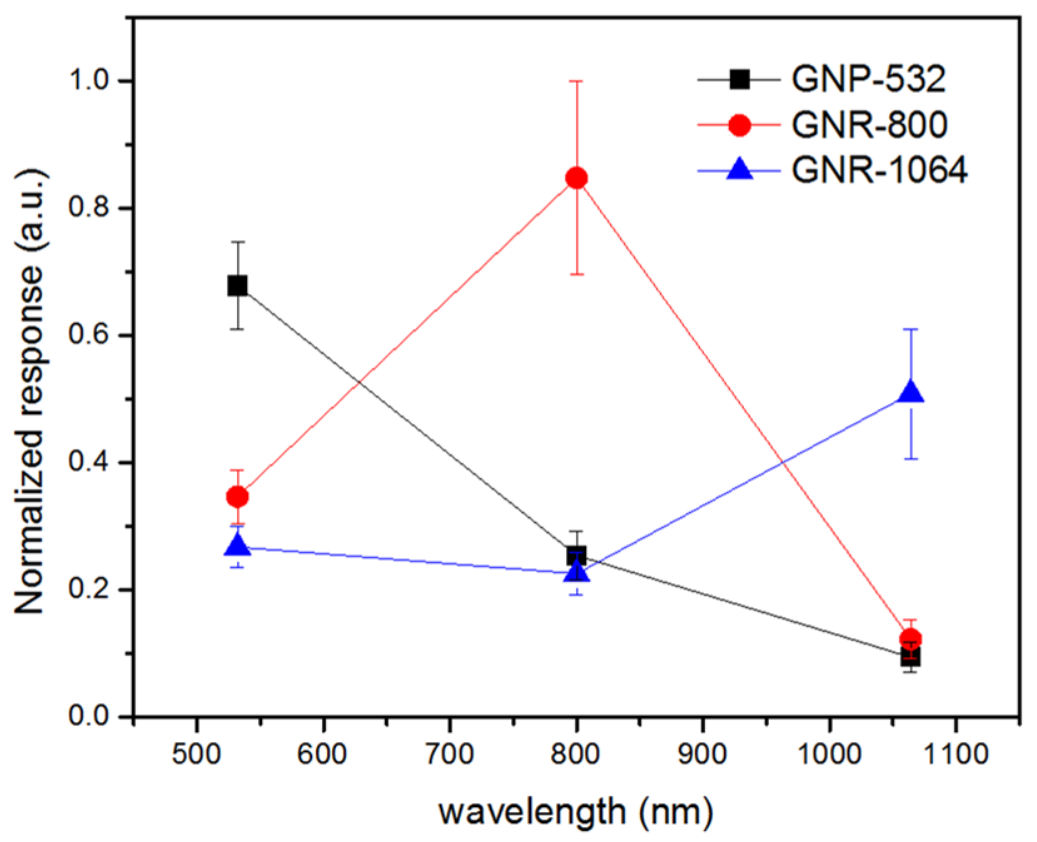

Figure 4.18: Normalized SAW-PA response of $40 \mathrm{~nm}$ gold nanoparticle (GNP-532), 10 $\mathrm{nm} \times 40 \mathrm{~nm}$ gold nanorod (GNP-800) and $10 \mathrm{~nm} \times 85 \mathrm{~nm}$ gold nanorods (GNP-1064) measured at three optical wavelengths- $532 \mathrm{~nm}, 800 \mathrm{~nm}$ and $1064 \mathrm{~nm}$.

acts as a resonator, with resonance along $\mathrm{X}$ and $\mathrm{Y}$-direction. The mode with an integer number of half-wavelengths generated in the X-direction within the PDMS microfluidic channel (as shown in Fig 4.19(b) is ideal for SAW excitation, due to the absence of bulk wave losses caused by wave refraction into the piezoelectric substrate.

\subsubsection{Finite element mode}

Fig 4.19(a) shows the 2D FEM geometry used to simulate the SAW displacements on the $\mathrm{LiNbO}_{3}$ substrate under PA excitation. Thermoacoustic module in COMSOL implements fully linearized compressible Navier-Stokes, continuity, and energy equations to obtain the acoustic pressure variations $p$, fluid velocity variations and acoustic temperature variations $T$. The input for the simulation is a constant heat source $Q$ (in frequency domain represents a wideband heat source) which generates an acoustic pressure wave. In case of the photoacoustics (PA), an incident laser pulse 


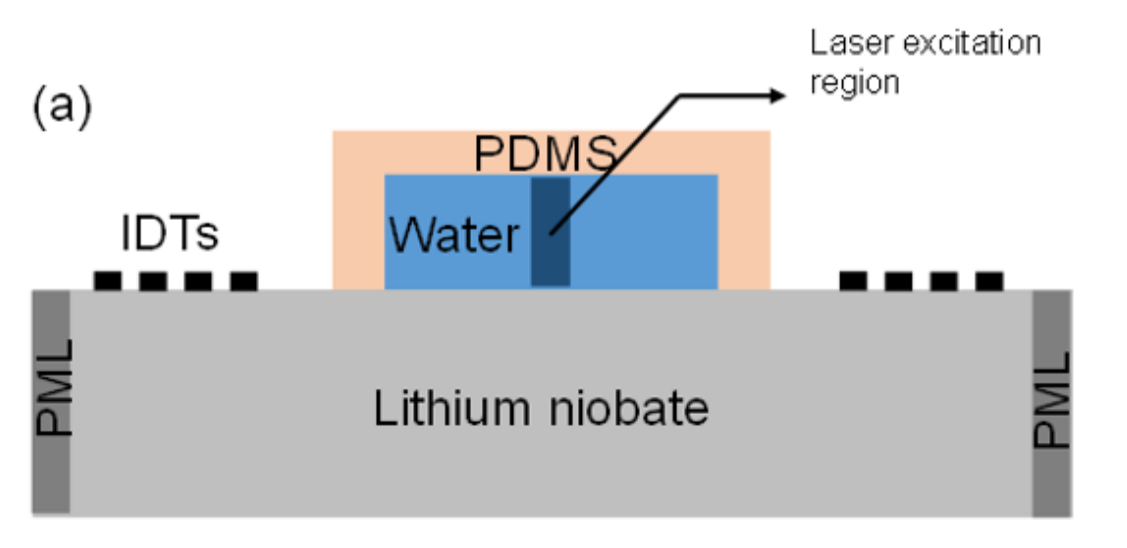

(b)

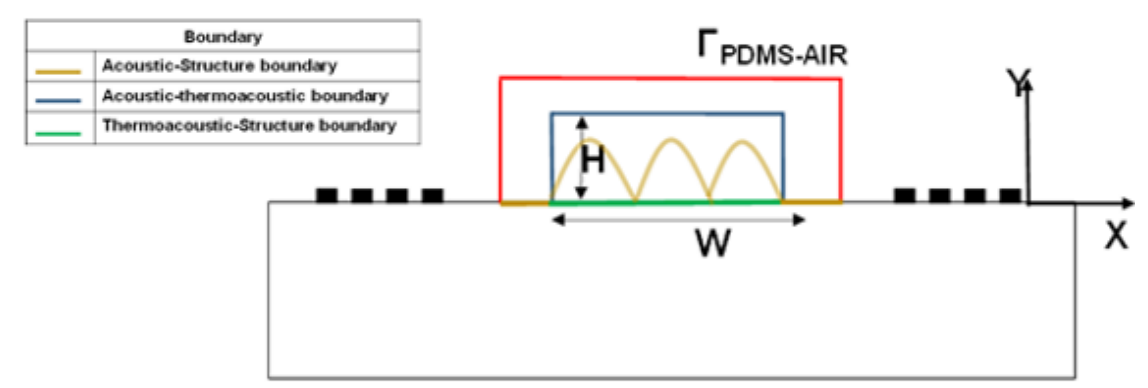

(c)

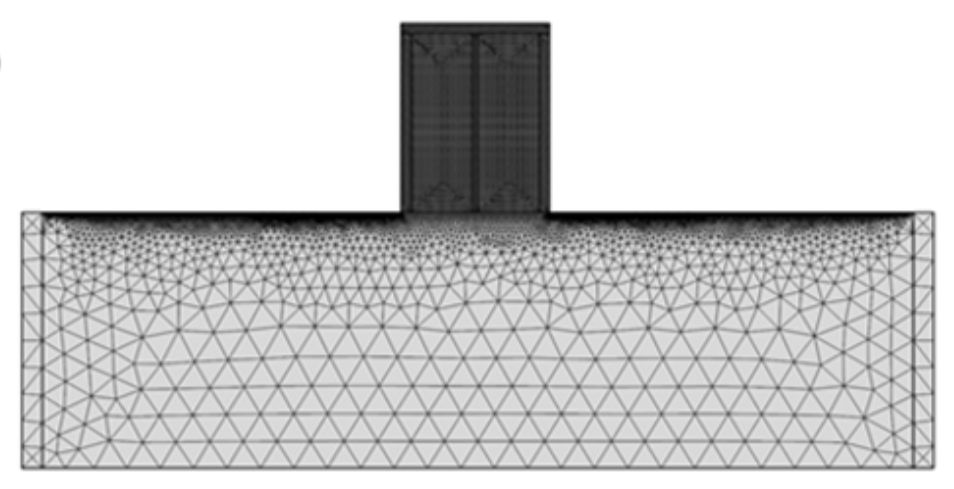

Figure 4.19: (a) 2D sketch of the SAW-PA sensor consisting of $\mathrm{LiNbO}_{3}$ substrate and fluid filled PDMS microfluidic channel. The center region of the fluid is the laser induced heat source. Note that the figure is not drawn to scale.(b) FEM computational domain with the boundaries. An acoustic mode (in yellow) with wave propagation along $\mathrm{X}$-direction is the optimal mode for SAW excitation.(c) FE mesh of the SAW-PA sensor

generates heat. The heat $Q$ acts as the source term for the energy equation and solves for the temperature $(\mathrm{T})$. Depending on the material properties such as thermal coefficient of volume expansion $(\beta)$ and isothermal compressibility $(\kappa)$, the tempera- 
ture change $(T)$ is converted to PA pressure (p), as shown by the Eq. 4.5 below.

$$
\frac{d V}{V}=-\kappa p+\beta T
$$

In this model, the nanosecond pulsed laser (1 ns) was approximated as an impulse excitation. This assumption explains the reason for applying a constant value as the heat source input for the thermoacoustics physics model. The heat source input of $1 \mathrm{~W} / \mathrm{m}^{2}$ was used for the thermoacoustic model. In this model, water was used as the fluid domain. The acoustic properties of water match closely to the dye used in the experiment. The default values specified in COMSOL for the material properties (water, PDMS) are applied in the simulation. The thermoacoustic properties for water calculated at $273^{\circ} \mathrm{C}$ is given in Table 4.2. The piezoelectric properties of $128^{\circ} \mathrm{YX}$ $\mathrm{LiNbO}_{3}$ were selected from the work done by Kannan [165]. Air-PDMS boundary was modelled as a sound soft boundary because the impedance condition follows $Z_{\text {air }}<<$ $Z_{\text {PDMS }}[166,167]$. Fig 4.19(b) shows the computational domain with the boundary conditions and domain interfaces. Three half-sinousoidal waves (in yellow) represents an acoustic mode $(0,3)$ with wave propagation along the $X$ direction. Perfectly matched layers (PML) were used to model an infinte extent of the substrate. The laser excitation domain was represented by a rectangular region in the centre of the fluid domain with a width of $100 \mu \mathrm{m}$. To keep the simulation computationally efficient, the thermoacoustic model for the fluid was coupled to pressure acoustics domain for the PDMS. A custom mesh with maximum element size equal to wavelength/8 (corresponding to a frequency of $100 \mathrm{MHz}$ ) was used to mesh the acoustic domain. The solid domain close to the IDT was modelled with a maximum element size of wavelength/5 [165]. Mesh refinement study was performed to confirm the accuracy of the selected meshes for the simulation. 


\begin{tabular}{|c|c|}
\hline Parameter & Value \\
\hline Coefficient of thermal expansion & $70 \times 10^{-6}[1 / \mathrm{K}]$ \\
\hline Specific heat at constant pressure, $C_{P}$ & $4216.6 \mathrm{~J} /(\mathrm{kgK})$ \\
\hline Ratio of specific heats, $\gamma$ & 1 \\
\hline Density, $\rho$ & $1003.9 \mathrm{~kg} / \mathrm{m}^{3}$ \\
\hline Speed of sound $v_{s}$ & $1403 \mathrm{~m} / \mathrm{s}$ \\
\hline
\end{tabular}

Table 4.2: Thermoacoustic properties of water at $273^{\circ} \mathrm{C}$

\subsubsection{Analysis of resonance modes}

The PDMS microfluidic channel acts as a resonance cavity for the lasergenerated acoustic wave. Multiple acoustic modes exist within the rectangular cavity. The resonance frequencies of a cavity with height $(H)$ and width $(W)$, is given by [168].

$$
f_{j}=\frac{c}{2} \sqrt{\left(\frac{l}{H}\right)^{2}+\left(\frac{m}{W}\right)^{2}}
$$

where, $l$ and $m$ represent the acoustic modes along the height and width of the cavity respectively. The resonant medium consists of PDMS and water. Frequency domain study was performed to determine the different acoustic modes and obtain the corresponding SAW displacements. Fig 4.20 shows the numerical simulation results obtained for a microfluidic channel of dimension $500 \mu \mathrm{m}$ (width) x $350 \mu \mathrm{m}$ (height). The PDMS wall had a sidewall thickness $\left(T_{s}\right)$ of $1 \mathrm{~mm}$. Fig $4.20(\mathrm{a})$ presents the frequency response of the displacement (refer to Fig 4.19(b) for the axis representation) along the Y-axis at a point on the surface of the piezoelectric substrate outside the channel area. The transverse displacement has a maximum amplitude at a frequency of 13.4 $\mathrm{MHz}$; the corresponding pressure profile is shown in Fig 4.20(b). The colour plot confirms that an incident pressure profile parallel to the substrate is generated. This mode favours the highest conversion efficiency (longitudinal to surface wave) without the usage of an external wedge element. Fig 4.20(c) represents the Y-axis displacement, which confirms the SAW excitation. 
(a)

(b)
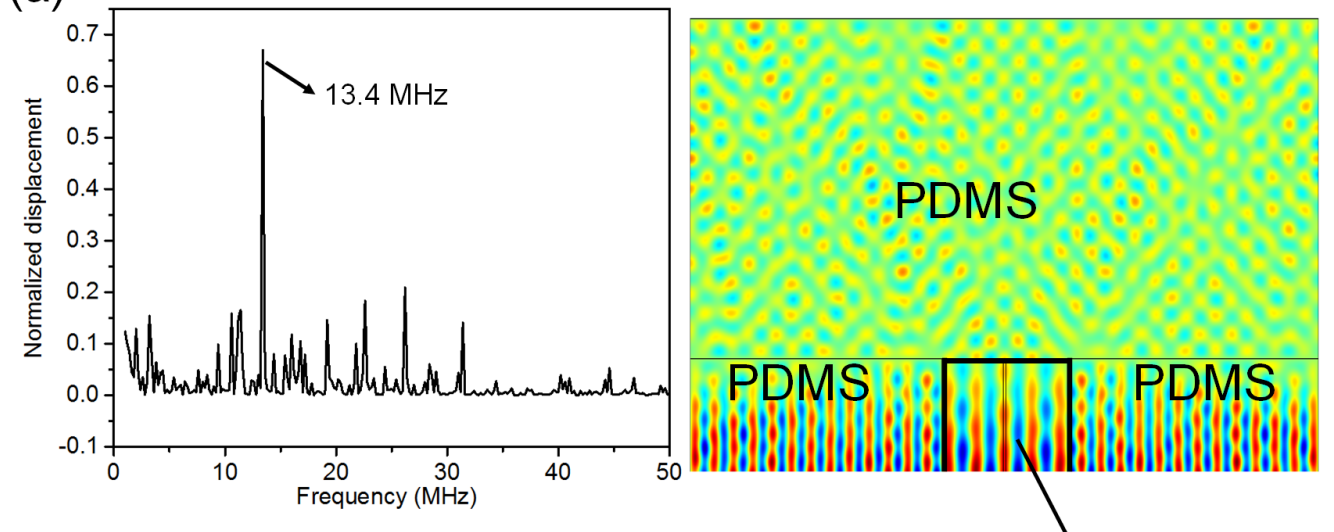

(c)

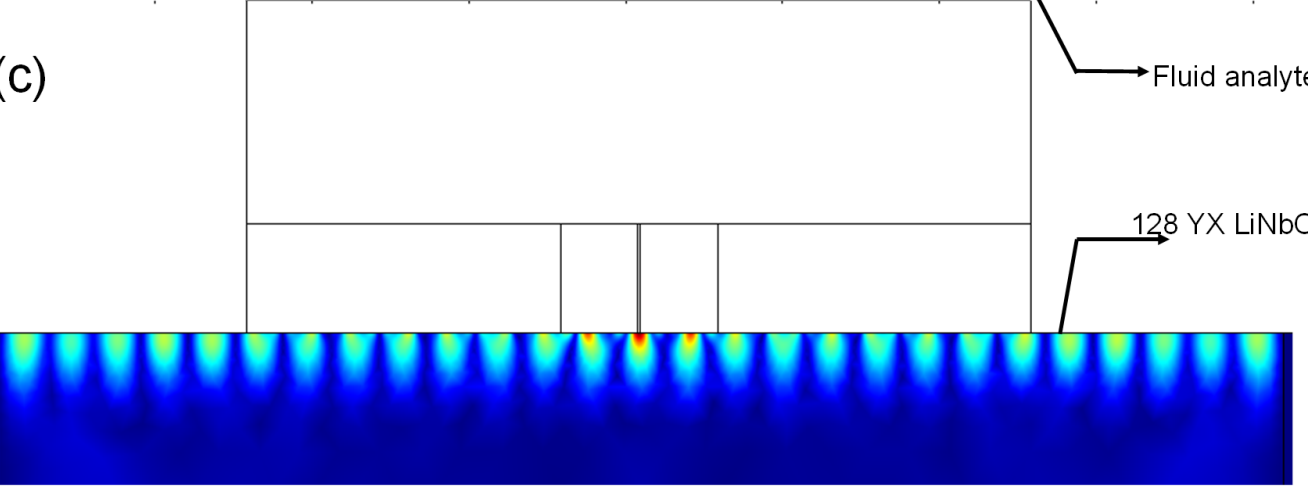

Figure 4.20: (a) Frequency spectra of the transverse displacement (Y-axis) on the surface of $\mathrm{LiNbO}_{3}$ at a point outside the microfluidic channel. Response at $13.4 \mathrm{MHz}$, corresponding to the frequency at which maximum displacement occurs:(b) Color plot of acoustic pressure inside the microfluidic channel (c) Color plot of the Y-axis displacement on $\mathrm{LiNbO}_{3}$.

\subsubsection{Effect of microchannel dimensions}

Considering the numerical complexity required for solving a large geometry, a smaller geometry was considered for the study with a PDMS side wall thickness of $100 \mu \mathrm{m}$. Fig 4.21(a) displays the frequency spectra for the Y-component of displacement at a point on the $\mathrm{LiNbO}_{3}$ surface using a PDMS microfluidic which follows the dimensions of Model A represented in Table 4.3. Fig 4.21(b) shows that the frequency of peak displacement $(7.6 \mathrm{MHz})$, the resonance mode is parallel to the $\mathrm{X}$-axis. 


\begin{tabular}{|c|c|c|}
\hline & $\begin{array}{c}\text { Channel } \\
\text { height }(\mathrm{H})\end{array}$ & $\begin{array}{c}\text { Channel } \\
\text { width(W) }\end{array}$ \\
\hline Model A & $350 \mu \mathrm{m}$ & $250 \mu \mathrm{m}$ \\
\hline Model B & $500 \mu \mathrm{m}$ & $250 \mu \mathrm{m}$ \\
\hline Model C & $350 \mu \mathrm{m}$ & $500 \mu \mathrm{m}$ \\
\hline
\end{tabular}

Table 4.3: Dimensions of the microfluidic channel

(a)

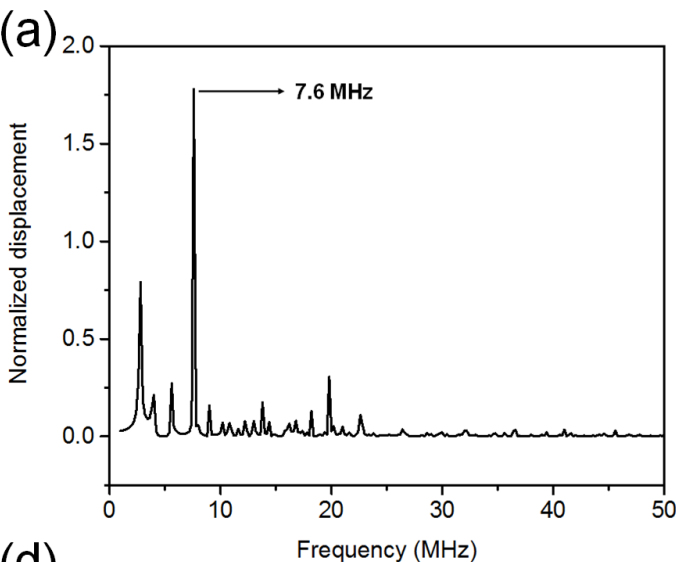

(d)

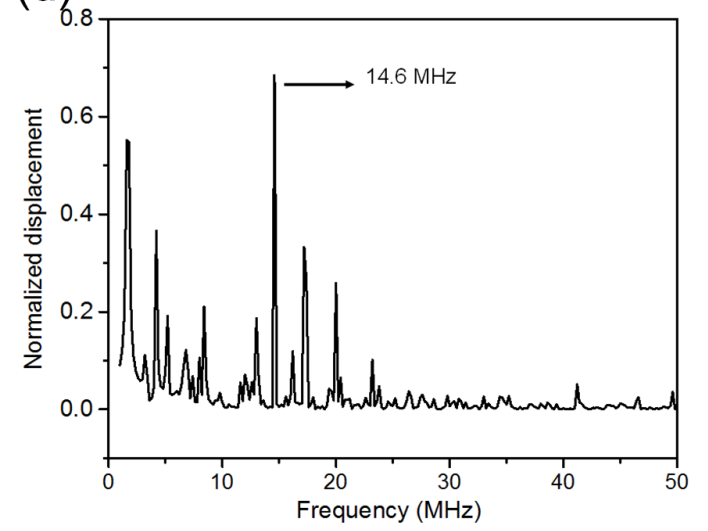

(b)

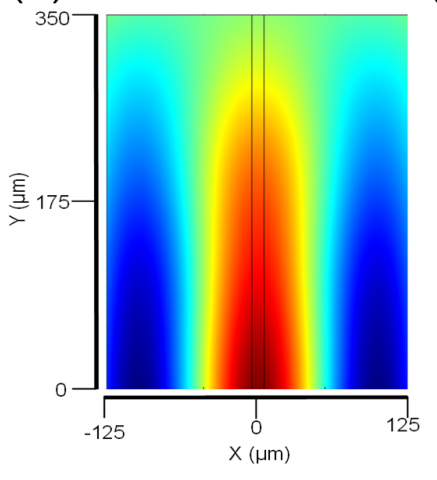

(e)

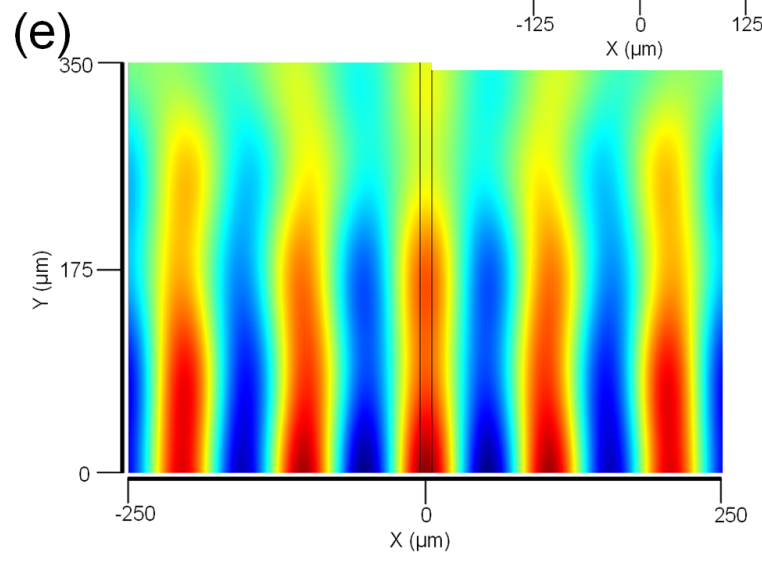

Figure 4.21: Frequency spectra of the transverse displacement (Y-axis) on the surface of $\mathrm{LiNbO}_{3}$ with a microfluidic channel of dimension shown in: (a) Model A, (d) Model $\mathrm{C}$ of Table 4.3. Color plots of the acoustic pressure in the microfluidic channel of dimensions: (b) Model A, (c) Model B and (e) Model C. 
Varying height of the microchannel Fig 4.21(c) depicts the pressure profile for the microfluidic channel of Model $\mathrm{B}$ at a frequency of $7.6 \mathrm{MHz}$. For Model $\mathrm{B}$, the width remains the same as Model $\mathrm{A}$, but the height is increased.Eq. 4.6 shows that for modes propagating parallel to the $X$-axis $(I=0)$, the resonant frequency will not vary with the height of the channel. The simulation results of Fig 4.21(c) prove that the zero-order modes parallel to the $\mathrm{X}$-axis will remain unaffected by changes in channel height.

Varying width of the microchannel The numerical simulation confirms that the variation in the width of the microfluidic channel affects the cavity modes. The maximum displacement for Model $C$ (different width and equal height as Model $A$ ) is obtained at a frequency of $14.6 \mathrm{MHz}$ (Fig 4.21(d)), with a higher order mode parallel to the $\mathrm{X}$-axis as shown in Fig 4.21(e). The study confirms that the resonant frequency can be tuned by varying the channel width and the PDMS side wall thickness (compare the side-wall thickness in Fig 4.20 and Fig 4.21).

\subsubsection{Experimental measurement}

\subsubsection{Experimental platform}

Experiment was conducted to assess the influence of appropriate acoustic frequency on the sensitivity of the SAW-PA sensor. As shown in Fig 4.22, it consists of a nanosecond pulsed laser of $1 \mathrm{~mJ}$ pulse energy focussed on the fluid contained in the PDMS microfluidic channel. The pulsed laser at a wavelength of $532 \mathrm{~nm}$ was illuminated at a repetition rate of $10 \mathrm{~Hz}$. The laser was focused into a spot-size of $100 \mu \mathrm{m}$ in the channel. Fig 4.22(b) shows the schematic of the microfluidic channel used in the experiment. The PDMS was trimmed along the width at the area overlapping the IDT. The PDMS side wall thickness $\left(T_{s}\right)$ is $1 \mathrm{~mm}$. The channel region has a width $(\mathrm{W})$ of $500 \mu \mathrm{m}$ and depth $(\mathrm{H})$ of $350 \mu \mathrm{m}$. The dimensions of the microfluidic channel used in the experiment follows the simulation shown in Fig 4.20. Carbon black ink 
is used as the sample fluid for the PA generation owing to its high optical absorption over a wideband. For this study, two-port SAW delay lines with resonant frequencies at $10 \mathrm{MHz}$ and $13 \mathrm{MHz}$ were used. Both the devices had 20 pairs of electrodes with a split-electrode configuration. The device was characterised using the E5061B ENA Series Network Analyzer. Fig 4.23(a) shows the measured S11 for the two devices. The signal obtained from the SAW device was amplified using a pre-amplifier (Panametrics 5077PR) with $49 \mathrm{~dB}$ gain, followed by data acquisition using an oscilloscope (wavesurfer from Lecroy) which was triggered by the laser illumination.

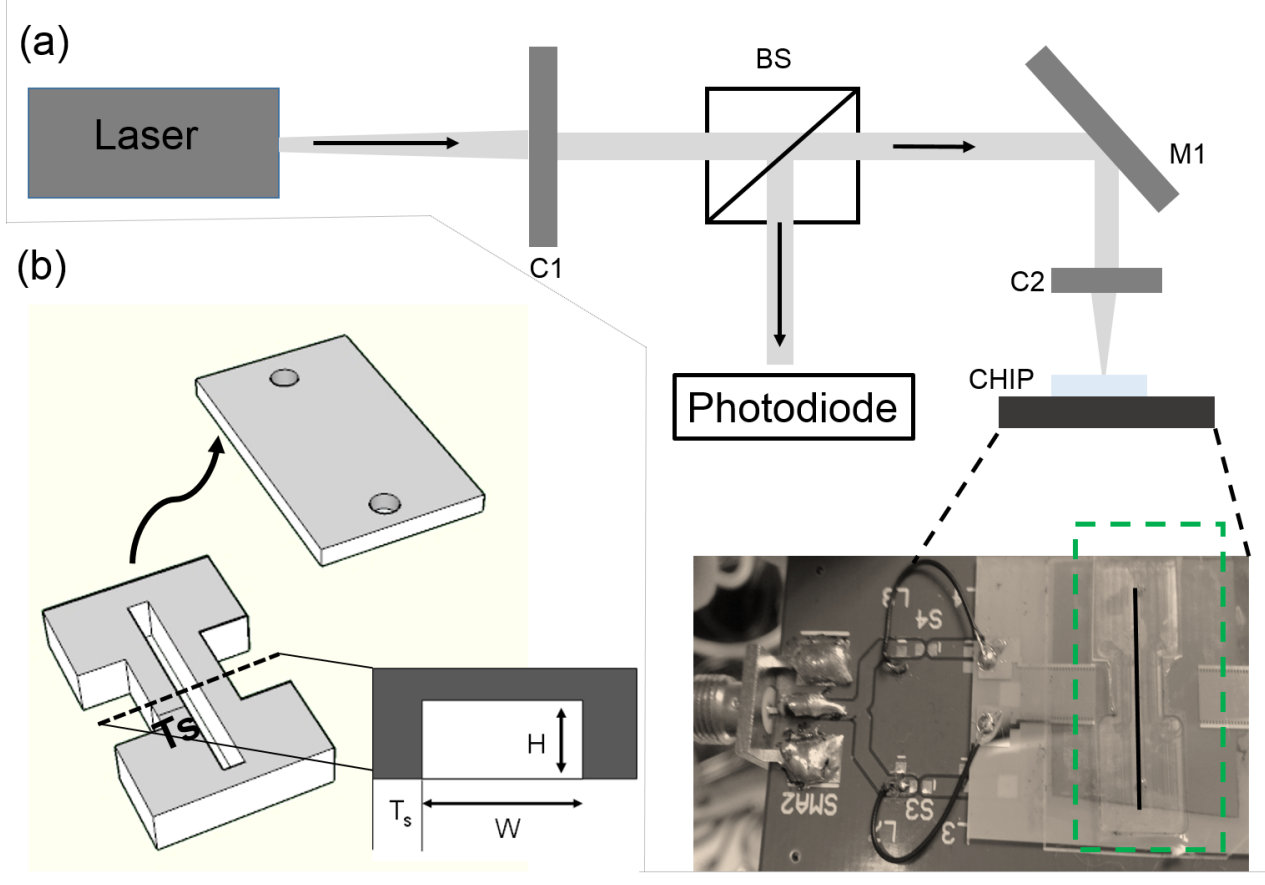

Figure 4.22: (a) The experimental setup for measuring the SAW-PA signal. C1: collimating lens; BS: beam splitter; M1: mirror; C2: objective lens. The SAW device with the microfluidic chip is zoomed to show the physical device. (b) Schematic of the microfluidic channel. For illustrating the channel, the top part of the microfluidic chip is separated. Note that the channel has been trimmed. Cross-section along the dotted line is shown, $T_{s}$ indicates the PDMS side wall thickness; $\mathrm{W}$ and $\mathrm{H}$ represent the width and height of the microfluidic channel respectively. 


\subsubsection{Frequency response of the SAW-PA sensor}

The accuracy of the numerical simulation was verified by comparing the sensitivity of the SAW-PA signals to different IDT frequency. According to the simulation results shown in Fig 4.20, the maximum surface displacement occurs at a frequency of $13.4 \mathrm{MHz}$. Hence selecting the SAW device with centre frequency at $13 \mathrm{MHz}$ is expected to give better sensitivity compared to the device at $10 \mathrm{MHz}$. Fig 4.23(b) shows the output voltage obtained from the two SAW devices. It is clearly observed from the voltage amplitudes that the SAW device at $13 \mathrm{MHz}$ has a higher sensitivity to the PA signal generated inside the microfluidic channel. The output signal was averaged over 20 sweeps and normalised to the laser intensity measured using a photodiode.

(a)

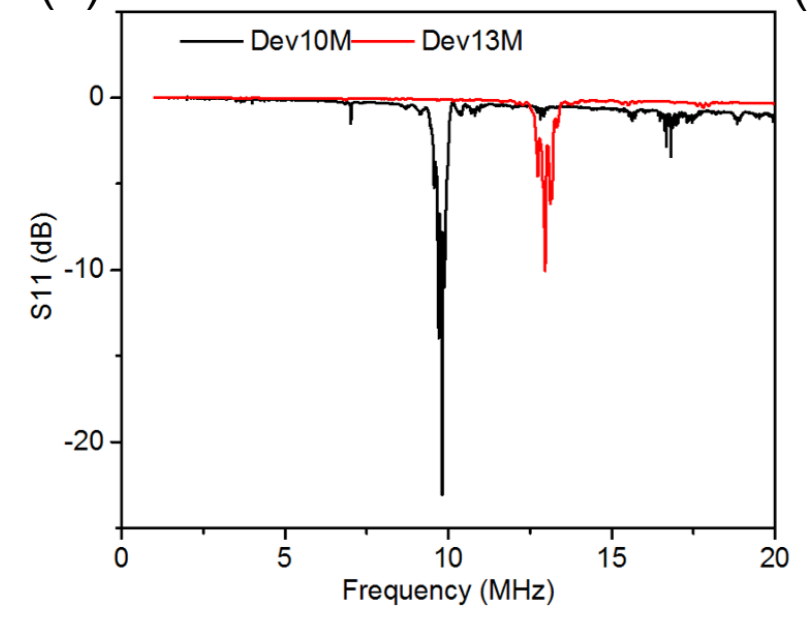

(b)

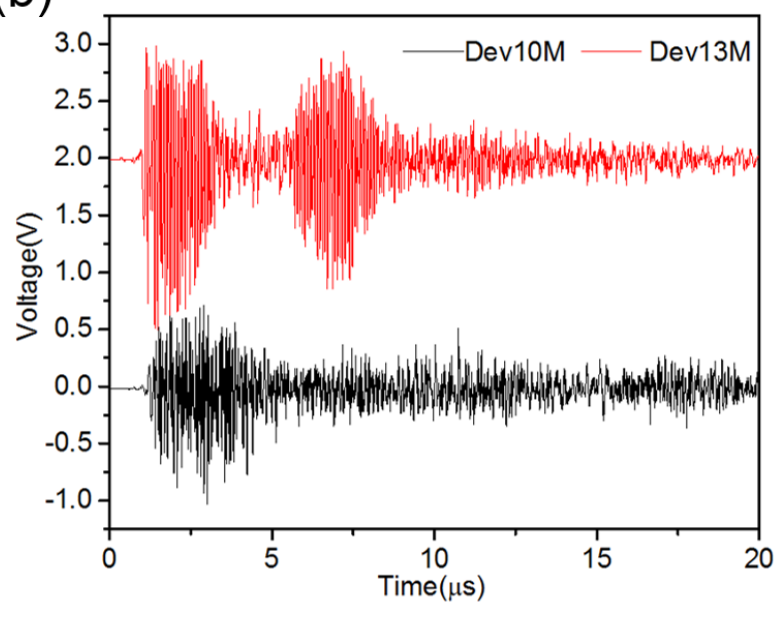

Figure 4.23: (a) Measured S11 of the SAW devices. The resonant frequency of the devices is $13 \mathrm{MHz}$ (Dev13M) and $10 \mathrm{MHz}$ (Dev10M). (b) Transient SAW-PA signals obtained from the SAW devices. Note that the signal from the Dev13M is shifted vertically by $2 \mathrm{~V}$ to improve the clarity. 


\subsubsection{Sensitivity calculation}

For the simulation model shown in Fig 4.20, the simulation results shows that for a heat input of $1 \mathrm{~W} / \mathrm{m}^{3}$, the output voltage obtained is $4 \times 10^{-14} \mathrm{~V}$. We consider a reference squaraine dye with applications in detecting analytes such as aminothiol in blood [169]. The optical absorption coefficient $(\alpha=\epsilon C)$ for the squaraine dye with molar extinction coefficient $(\epsilon)$ of $10^{5} \mathrm{M}^{-1} \mathrm{~cm}^{-1}$ and a concentration $(C)$ of $1 \mathrm{nM}$ is 10 ${ }^{-4} \mathrm{Cm}^{-1}$. The heat generated per unit volume $(\mathrm{Q})$ by a pulsed laser of $1 \mathrm{~ns}\left(\mathrm{t}_{\mathrm{s}}\right)$ duration is given by:

$$
Q=\alpha F / t_{s}
$$

where $\mathrm{F}$ is the fluence of the laser(=Energy / irradiated area). For a laser energy $1 \mathrm{~mJ}$ and laser spot size of $100 \mu \mathrm{m}$ (diameter), the heat generated $=1.27 \times 10^{10} \mathrm{~W} / \mathrm{m}^{3}$. On multiplying the heat $(Q)$ with the output voltage generated by the heat input of $1 \mathrm{~W} / \mathrm{m}^{3}$, an electrical potential of $508 \mu \mathrm{V}$ is obtained. We have verified that our model is linear, with the heat input linearly proportional to the electrical potential. Thus, the device has a sensitivity of $508 \mu \mathrm{V} / \mathrm{nM}$ at $13.4 \mathrm{MHz}$. The use of a narrow band SAW-IDT designed to the $13.4 \mathrm{MHz}$ reduces noise and hence becomes a very sensitive detection method.

\subsection{Conclusion}

In conclusion, we demonstrate a novel integrated SAW-PA sensor platform for a couplant-free, simultaneous optical and mechanical characterization of fluids flowing through a microchannel. The PA generated longitudinal acoustic wave is mode converted into Rayleigh SAW at the interface of the liquid- $\mathrm{LiNbO}_{3}$ substrate. By exploiting the piezoelectric substrate as the medium for acoustic wave propagation, the requirement of a coupling layer (water) for the ultrasound transducer was eliminated in this platform. Using analytical modelling, we show that a high mode conversion efficiency of 80 percent is possible by optimal designs of the SAW-PA device. The optical charac- 
terization capability of the SAW-PA device was demonstrated by performing the optical absorption spectroscopy and measuring the concentration of the fluid sample in the microchannel using two standard dye solutions. We further investigated the effects of viscosity on the SAW-PA signal to exhibit the ability of the platform to detect mechanical parameters of the fluid, which is otherwise difficult to detect using standard optical protocols. The sensor was also successfully applied to study the SAW-PA response of plasmonic nanoparticles by selecting various sizes of GNPs and GNRs as a model system. The sensitivity to the nanoparticles size and shape was also illustrated. In future, the SAW-PA platform can also be applied for bio/chemical sensing by detecting the shift in optical absorption spectra, which is induced by the receptor-analyte binding. Further, we implemented a finite element model for the SAW-PA sensor using COMSOL multiphysics 4.4. The PDMS microfluidic channel behaves as a rectangular resonator with multiple modes along the width and height of the channel. The modelling results show that maximum mode conversion efficiency (longitudinal to surface acoustic wave) is obtained at a frequency in which the pressure is directed parallel to the piezoelectric substrate. SAW devices designed at this frequency will have the highest sensitivity to the PA signal inside the microfluidic channel. The resonant behaviour of the PA signal in a microfluidic channel makes the high Q SAW device potentially an optimal sensor for detecting PA waves induced in a microfluidic channel. In conclusion, we show that the wideband PA signal is converted to a narrowband signal due to resonance inside the microfluidic channel and existence of modes parallel to the substrate, satisfies the condition for maximum acoustic coupling to SAW and alleviates a wedge structure 


\section{Chapter 5}

\section{Integrated microfluidic system using}

\section{SAW for real-time separation and \\ detection of particles on a single}

\section{substrate}

In this chapter, we introduce a microfluidic system seamlessly integrating separation, concentration, and detection of particles on a single piezoelectric (128 $\left.\mathrm{YX} \mathrm{LiNbO}_{3}\right)$ chip. The existing capabilities of the standing surface acoustic waves (SSAW) for highefficiency separation of particles and cells is utilised along with a single IDT based particle aggregation to preconcentrate the sample before sensitive detection. The detection scheme involves sensing the surface acoustic waves (SAW) generated by the optically induced mechanical resonance of the photoacoustic(PA) wave produced by the particles inside the microfluidic cavity. As a proof of concept study, the developed integrated microfluidic system was applied to separate 3- and 10- $\mu$ m-diameter polystyrene particles with an efficiency of separation above $95 \%$. The separated 10 
$\mu m$ particles were directed into the sensing chamber, where a single SAW was used to concentrate the particles before real-time quantitative detection. The sensor presented a reliable $\left(R^{2}=0.98\right)$ detection of the $10 \mu \mathrm{m}$ particles down to 7 particles in 10 $\mu L$ of the sample volume in 15 minutes.

\subsection{Introduction}

Emerging technologies utilising micro and nanoscale engineering have profound applications in the health and point of care theranostics. Therefore, developing such low-cost diagnostic devices which follow a rapid and sensitive detection protocol receives prime attention. Currently available rapid diagnostic tests (RDTs) which possess cheaper and quick response time are limited mainly by the low detection limit (for example 100 parasites/L) and lack of quantification [170]. On the other hand, sensitive and selective detection methods such as polymerase chain reaction (PCR) and enzyme-linked immunosorbent assay (ELISA) require expensive equipment and trained professionals to perform the tests. In this context, integrated microfluidic systems combining microfluidic actuation with various sensing technologies to realise point-of-care (POC) devices is essential [171-173]. In addition to the benefits of miniaturisation, such devices offer low power consumption profile with higher sensitivity. Kong et al. [173] demonstrated the use of deformability separation techniques to enrich infected red blood cells and magnetic resonance relaxometry sensing for early malarial detection at a level of $0.0005 \%$. Similarly, Tokel et al. [171] demonstrated the merging of microfluidics with plasmonic sensing approaches to realise a label-free detection platform. Thus, among other parameters, sample enrichment and detection are vital steps to accomplish an efficient integrated microfluidic system.

In the field of microfluidics, surface acoustic wave (SAW) technology has been receiving much attention over the past several years [97, 99, 174, 175]. The nanometer scale vibrations excited by metal electrodes on piezoelectric substrate 
$\left(128^{\circ} \mathrm{YX} \mathrm{LiNbO} 3\right)_{3}$ couples to the fluid and perform various microfluidic manipulations. Exposing a droplet to an asymmetric SAW distribution causes rapid (in few seconds) particle/cell concentration within it. The interaction of acoustic streaming and acoustic radiation forces causes the particles to aggregate within a droplet $[176,177]$. The technique was utilised by performing nanoparticle aggregation within the solution to aid surface-enhanced Raman scattering (SERS) detection [178]. Furthermore, the primary acoustic radiation force (ARF) exerted by the standing surface acoustic wave (SSAW) was utilised for high-efficiency separation of particles and cells. Reported results $[52,100]$ demonstrated segregation of particles with $97-99 \%$ efficiency and rare cancer cells from normal WBCs with a recovery rate over 83\% [45]. The separation performance can be further improved by optimising the flow rate, input power and channel dimensions. Though many research works were focussed on separating rare clinical samples from the bulk using SAW, the downstream detection still relies on microscopes and cell counters like haemocytometers which have been performed off-chip [52]. Sample loss while transferring between successive stages is the risk associated with such schemes [179]. In this scenario, a microfluidic platform integrating the high-efficiency SAW separation and real-time quantitative detection is seldom reported, which could be useful for segregate and quantitatively detect bio-relevant substrates.

In this Chapter, we present a SAW based integrated microfluidic platform for sample separation, concentration and detection. In this platform, a mixture of two different particle sizes is first introduced into a SAW separation stage. Particles with larger size are separated and transported by the flow into the outlet chamber for detection. The response time and sensitivity of the sensor were improved by utilising the SAW to induce particle concentration in the microfluidic reservoir. Further, the pulsed laser was irradiated on the concentrated particles for detection. The sensing principle relies on our recently developed optically induced acoustic resonant sensing platform: photoacoustic (PA) wave induced SAW (SAW-PA). [153] The integrated platform was 
used to demonstrate the separation of polystyrene beads of two different sizes 3- and $10-\mu \mathrm{m}$-diameter. The separated $10 \mu \mathrm{m}$ particles inside the microfluidic cavity were detected using the SAW-PA sensor. The sensor detected 7-200 particles inside a $10 \mu \mathrm{L}$ of fluid in 15 mins.

\subsection{Materials and methods}

\subsubsection{Device design and operation}

A schematic of the integrated microfluidic platform is shown in Fig 5.1. The integrated device is structurally divided into three parts. The first part is a piezoelectric substrate $\left(128^{\circ} \mathrm{YX} \mathrm{LiNbO}_{3}\right.$, lithium niobate) which encompasses the metal electrodes (IDT, interdigital transducers) for the actuation and sensing. The second part consists of a PDMS microfluidic channel. The main channel is $200 \mu \mathrm{m}$ high and $200 \mu \mathrm{m}$ wide. The PDMS channel is bonded to the substrate using oxygen plasma. The third part consists of a $532 \mathrm{~nm}$, nanosecond (1nsec) pulsed laser that operates at a pulse repetition frequency of $10 \mathrm{~Hz}$. The laser irradiates on the separated particles to excite the SAW-PA, which is detected using the sensing IDTs. The pulsed laser illuminates the sensing chamber from the top with a laser fluence of $31 \mathrm{~mJ} / \mathrm{cm}^{2}$ for $200 \mu \mathrm{m}$ spot size. An optical microscope was used to visualise the particles inside the sensing chamber and a translation stage allowed the device to be moved to the site for the laser irradiation. The pulsed laser was split into two outputs using an optical fibre and focussed into the active $(G)$ and reference $(E)$ sensing cavities. The laser was targeted to the concentrated particles using a mechanical scanner. The SAW device was held on a platform and translated in the horizontal direction by using two DC servo motors (KDC101, Thorlabs) for the $X$ and $Y$ directions. The step size of each scan points was set to $50 \mu \mathrm{m}$. Two stages of low noise amplifier (ZFL-1000LN+) were cascaded to obtain a gain of $40 \mathrm{~dB}$ gain. Each output from the IDTs was connected to the amplifier, 
followed by data acquisition using an oscilloscope (WaveSurfer from Lecroy).

a)
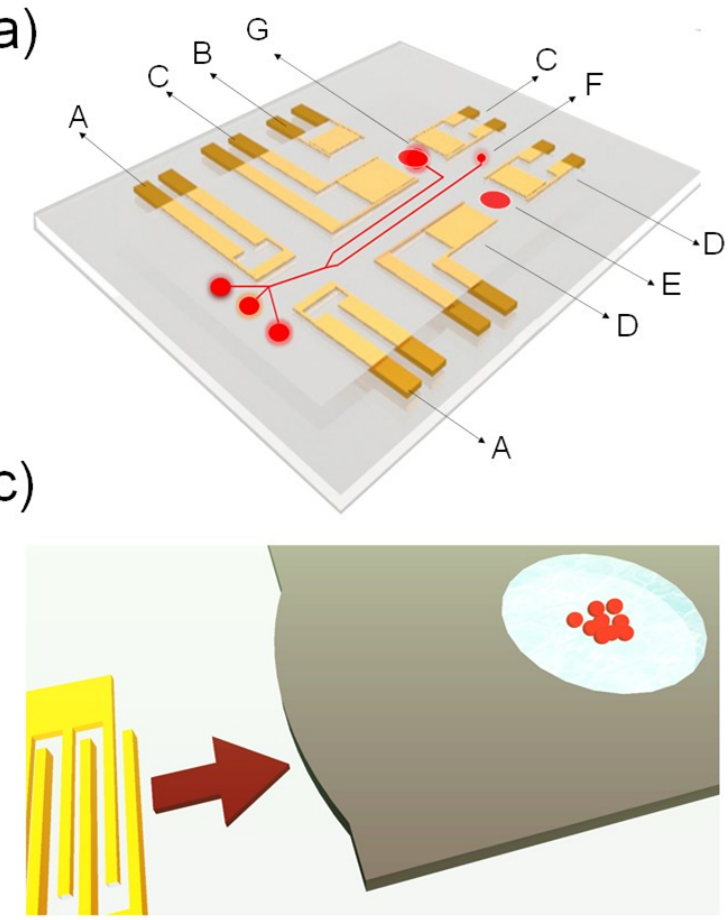

b)

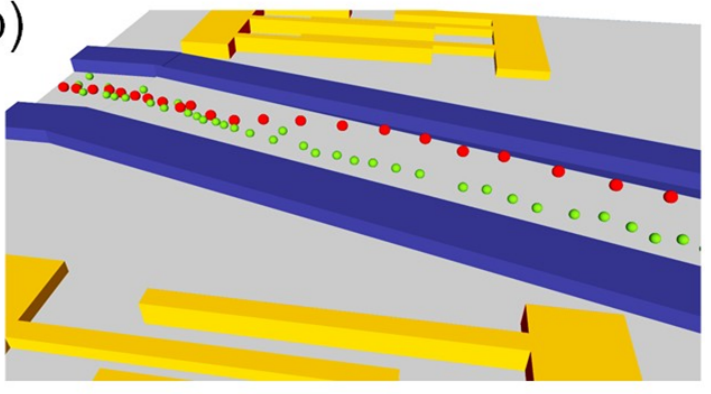

d)

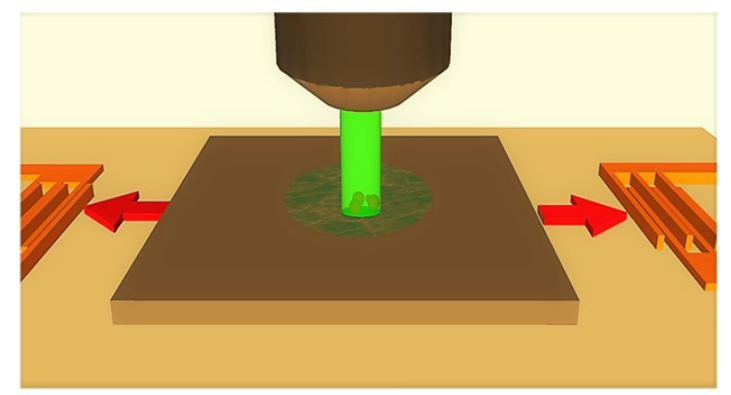

Figure 5.1: (a) Schematic diagram of the SAW-PA integrated microfluidic device. The particles are injected through the centre inlet. The sheath flow through the outer inlets focuses the particles in the main channel. The particles of two different sizes (3- and $10 \mu \mathrm{m})$ are separated using the Tilted-angle standing surface acoustic wave (taSSAW) generated by interdigital transducer (IDTs)- $A$. The larger particles flow downstream into the sensing reservoir- $G$ while the smaller particle moves to the waste reservoir- $F$. $E$-denotes the reference cavity, which is filled with DI water. The IDT- $B$ concentrates the particles $(10 \mu \mathrm{m})$ inside the sensing cavity. Further, the pulsed laser irradiation on the sensing and reference reservoir generates a photoacoustic (PA) signal, which resonates inside the polydimethylsiloxane (PDMS) reservoir and mode converts into a SAW. The IDTs- $C$ (active) and $D$ (reference) detects the PA-induced SAW. (b) The IDTs inclined at an angle $\left(15^{\circ}\right)$ to the direction of fluid flow, used to separate the particles (red and green coloured spheres inside the channel represent the large and small sized particles respectively). (c) The IDTs produces SAW that interacts with the particles inside the reservoir and concentrates them. (d) The laser irradiates on the particles to generate a SAW, which is detected by the IDT 


\subsubsection{Experimental setup for the sensing stage}

The experimental setup for the sensor is illustrated in Fig 5.2. The pulsed laser is set to the external trigger mode. A personal computer (PC) provides a trigger signal to the pulsed laser and the DC servo motors (controlling the mechanical scanning stage). The laser synchronisation signal triggers the oscilloscope to acquire the SAW-PA signal obtained after amplification. The raw data received from the oscilloscope is stored on the PC. The device is translated in the horizontal direction by the scanner to the laser scanning region for sensing. A combination of a collimator lens (C1), neutral density filter (NDF) and a condenser lens (Conl) is used to focus the light into the optic fibre. A microscope working in the reflection mode is used to observe the particles inside the reservoir.

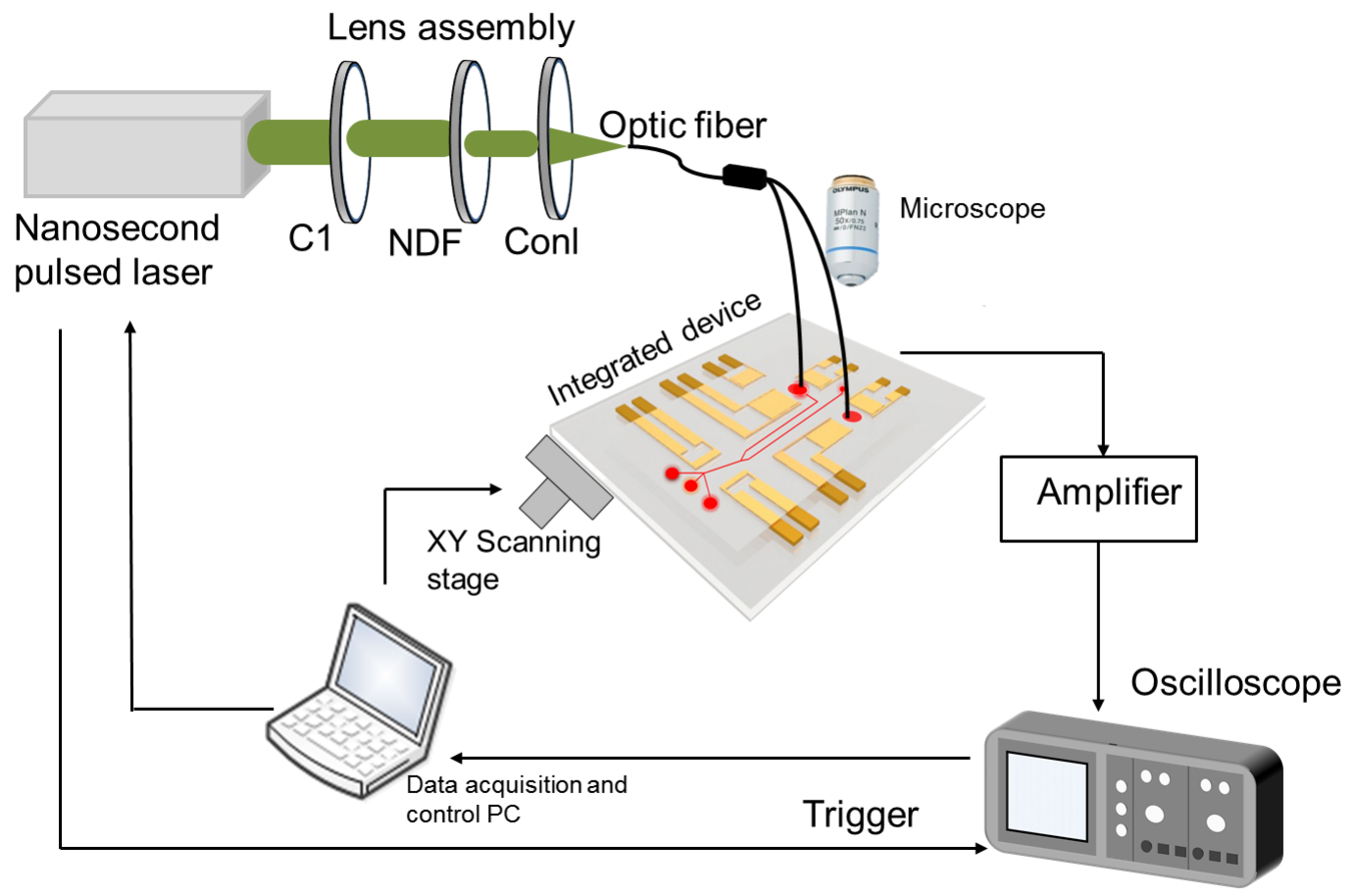

Figure 5.2: Schematic of the experimental system used for SAW-PA sensing of particles 


\subsubsection{SAW device fabrication}

The fabrication of SAW device involves photolithography, metal deposition and lift-off process. The lithium niobate wafer was first treated in a hexamethyldisilazane (HMDS) oven to promote photoresist (PR) adhesion. A layer of PR (AZ9260, Microchem) was spin-coated on the wafer, placed in a hot-plate (for two mins) and then exposed to a UV light source to develop the pattern. The patterned wafer is dipped in a PR developer solution (AZ400K). A double layer of metal ( $\mathrm{Cr} / \mathrm{Au}, 20$ $\mathrm{nm} / 200 \mathrm{~nm}$ ) was deposited on the wafer using magnetron sputtering. Subsequently, the wafer was dipped in acetone for lift-off and forms the IDT.

\subsubsection{SAW device characterization}

The frequency response of the fabricated SAW IDTs is measured using the E5061B ENA Series network analyser. The S-parameters obtained for the different sets of IDTs in the integrated device are shown in Fig 5.3. Fig 5.3 - (a) and (b) shows the S11 measurement obtained for a single port of the IDT used for separation and concentration respectively. Fig 5.3(c) and 5.3(d) shows the S11, S12 and S22 measurement for one pair of sensing IDTs designed for $10 \mathrm{MHz}$ and $235 \mathrm{MHz}$ centre frequencies

\subsubsection{Functionality of the integrated device}

A single SAW substrate incorporates the actuation and sensing functionalities. Functionally; the SAW device is divided into three stages.

\subsubsection{Design of the separation stage}

The first part consists of a Tilted-angle standing surface acoustic wave (taSSAW) [52] for high-efficiency separation of particles based on their difference in size. 

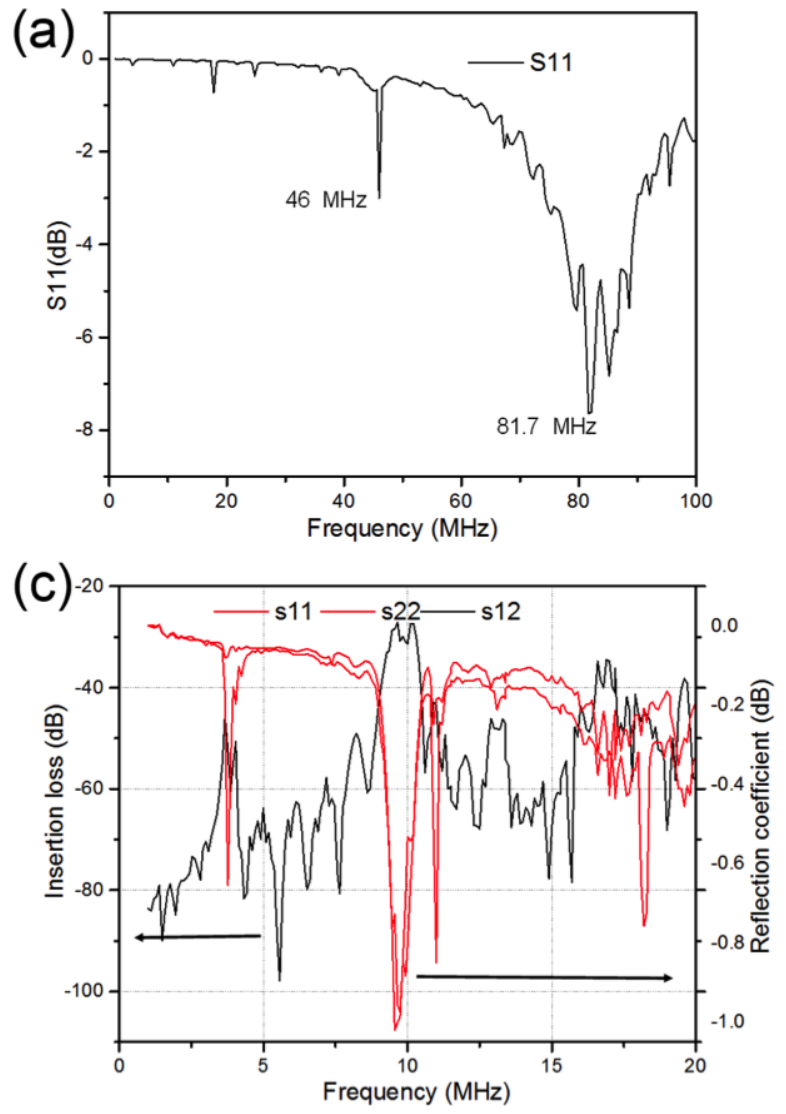
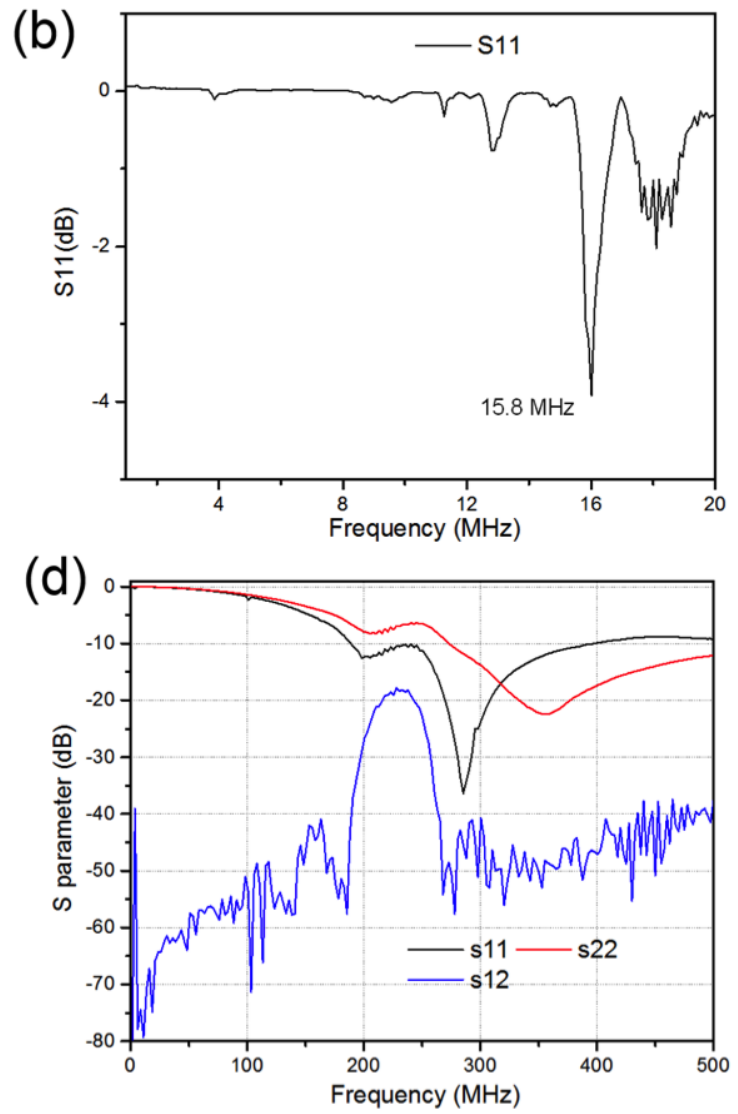

Figure 5.3: S-parameter obtained for different SAW devices. (a-b) corresponds to the S11 parameter of the SAW device used for separation and concentration stage respectively. (c-d) shows the reflection coefficients (S11, S22) and insertion loss (s12) of the devices for sensing at $10 \mathrm{MHz}$ and $235 \mathrm{MHz}$ respectively.

The IDTs had ten pairs of electrodes with constant electrode spacing and fingeroverlap of $40 \mu \mathrm{m}$ and $2.5 \mathrm{~mm}$ respectively. The SAW propagates in an orthogonal direction to the usually preferred X-propagation direction of the substrate as shown by the IDT-A in Fig 5.1(a). The sound speed in this direction is slightly lower and equals to $3680 \mathrm{~m} \mathrm{~s}^{-1}$ [180]. According to the design, the fundamental frequency for SAW generation exists at $46 \mathrm{MHz}$ which is observed in the S11 plot (see Fig 5.3(a)). However, at $81.7 \mathrm{MHz}$ there exists a stronger resonance peak. This frequency is selected for the separation experiment. Coherent AC signals which were generated by an RF signal generator (Tektronix, AFG 3252) and amplified by a power amplifier (Minicircuits, 
ZHL-100W-GAN+) were applied to the IDTs to form a standing surface acoustic wave (SSAW) within the microfluidic channel. The input power was set to $24 \mathrm{dBm}$. The IDTs were oriented at an angle of $15^{\circ}$ to the flow direction through the main channel. A schematic of the separation stage is shown in Fig 5.1(b). Particles of size 10- and $3-\mu \mathrm{m}$ were introduced into the central inlet, and they were converged towards the centre of the main channel by the exterior sheath flows. The particles were introduced into the main channel at a flow rate of $\sim 1.2 \mathrm{~mm} / \mathrm{s}$ Continous flow was generated by connecting the inlet to syringes driven by NE-1000 syringe pumps.

\subsubsection{Design of the particle concentration stage}

The taSSAW separated particles migrated towards the sensing and the waste outlets. The larger particles collect in the sensing outlet. The flow was cut-off once the sensing outlet was filled with $\sim 10 \mu \mathrm{L}$ of sample volume. Before sensing, the particles were concentrated inside the sensing chamber using a single SAW IDT, which forms the second part of the integrated device. Fig 5.1(c) shows the schematic representation of the particle concentration in a fluid filled cavity using a single SAW IDT. The IDT used for aggregation consists of ten pairs of electrode with a pitch (p) and width of $199 \mu \mathrm{m}$ and $4 \mathrm{~mm}$ respectively. The SAW propagates in an orthogonal direction to the usually preferred X-propagation direction of the substrate as shown by the IDT-B in Fig 5.1 (a). The SAW device was excited at a resonant frequency of $15.8 \mathrm{MHz}$ (refer Fig 5.3(b) for the $s 11$ response of the IDT). The IDT was excited with a driving stage similar to that used for the taSSAW. Following the theoretical explanation published in work by Destgeer et al. [177], a standing surface acoustic wave (SSAW) is critical for particle concentration. The SAW attenuation length $\left(x_{s}\right)>$ fluid size (radius, $r_{d}$ ) should be satisfied for attaining particle concentration. This condition ensures that the travelling wave from the IDT reflects back from a boundary before it attenuates. The back-propagating wave interferes with the forward travelling wave to generate an SSAW. Following the design criteria mentioned above (refer to Fig 5.4 for the dimensions and design of the 
open-chamber), fluid was filled in an open-chamber surrounded by polydimethylsiloxane (PDMS). The PDMS microfluidic channel had an air-cavity surrounding the fluid in the chamber. The cavity acts as a boundary and ensured that the surface waves gets reflected back before it traverses a distance more than or equal to the attenuation length $\left(x_{s}\right)$. The attenuation length on the substrate at it interacts with the fluid is given by $x_{s}=\rho_{s} c_{s} \lambda_{s} / \rho_{f} c_{f}$ where $\rho_{s}=4630 \mathrm{~kg} \mathrm{~m}^{-3}$ and $\rho_{f}=998 \mathrm{~kg} \mathrm{~m}^{-3}$ are the densities of the substrate $\left(\mathrm{LiNbO}_{3}\right)$ and water respectively; $c_{s}=3680 \mathrm{~m} \mathrm{~s}^{-1}$ and $c_{f}=1480 \mathrm{~m} \mathrm{~s}^{-1}$ are the sound speeds in the substrate and water respectively and $\lambda_{s}$ is the wavelength of the acoustic wave in the substrate. At a frequency of $15.8 \mathrm{MHz}$, the attenuation length $\left(x_{s}\right)=2.7 \mathrm{~mm}$. The cavity was designed such that the surface waves encountered a soft boundary condition (PDMS-air interface) at a distance of $2.6 \mathrm{~mm}$. The equivalent $r_{d}$ is $1.3 \mathrm{~mm}$. The wave reflects before travelling its attenuation length, and hence a standing wave is generated in the fluid-filled open cavity to concentrate the particles. Additionally, the $k$-factor defined by $k=\pi d_{p} / \lambda_{f}$, where $d_{p}$ is the diameter of the particle and $\lambda_{f}$ is the acoustic wavelength in the fluid, has a value of 0.3. The condition that $k<1$, to create particle concentration in the centre of the fluid is also satisfied. Hence, the design of the open-cavity satisfies the two conditions that cause particle concentration in the centre of the sessile droplet. The validity of the design criteria mentioned above is further confirmed by performing the particle concentration experiment with the microfluidic channel having an open cavity of a larger diameter ( 5 $\mathrm{mm}$ ). The acoustic attenuation length was less than the cavity dimension. Following the theory explained above, the particles should not concentrate.

Microfluidic cavity design for SAW concentration: The cavity aims to meet the conditions for generating standing wave to cause particle concentration. Fig 5.4 depicts the channel design with the sensing and reference cavity. The cavity design ensures that the travelling surface acoustic wave (TSAW) reflects from a boundary at a distance $(2.6 \mathrm{~mm})$ less than its attenuation length $(3.2 \mathrm{~mm})$, thus forming a standing 
acoustic wave inside the sensing cavity

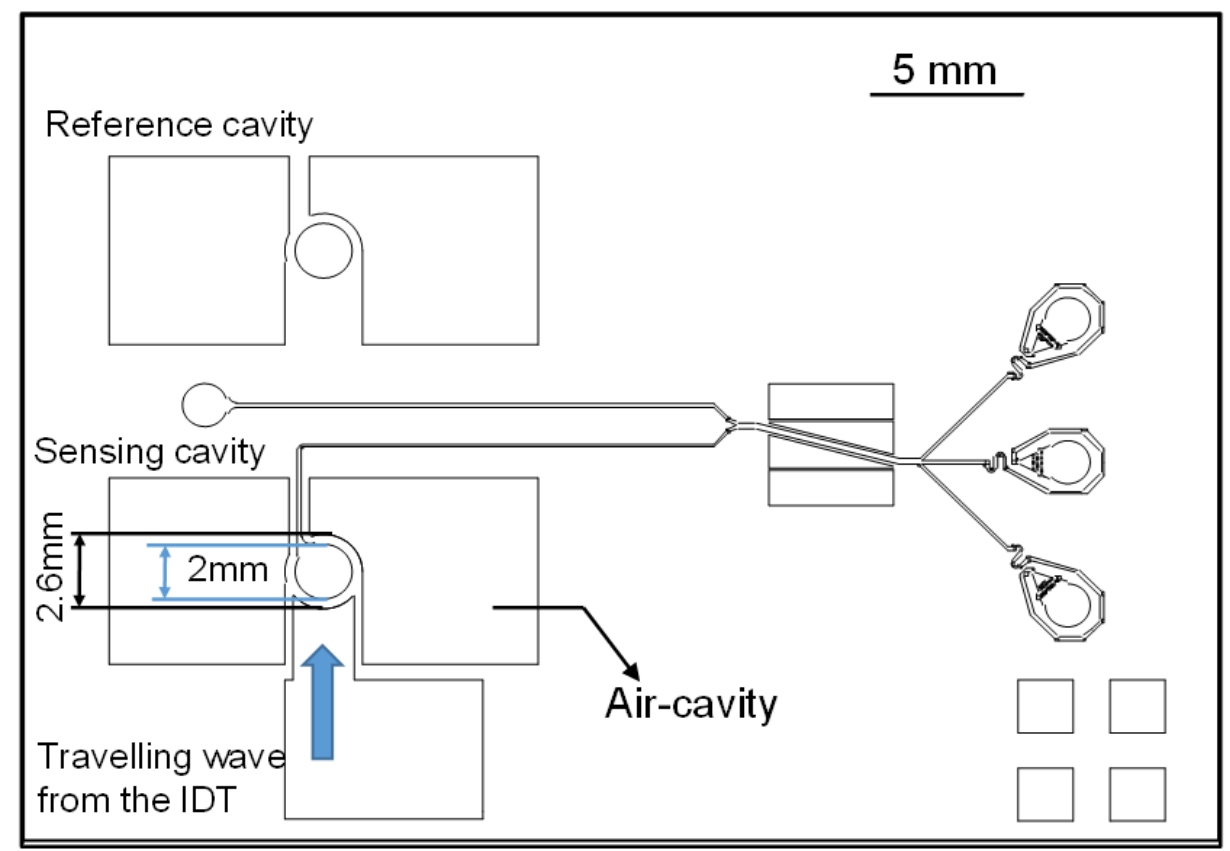

Figure 5.4: The design of the microfluidic channel. The boxes represent air-cavity (not filled with PDMS), only one of them is highlighted. The travelling surface acoustic wave(TSAW) from the concentrator IDT travels in the direction shown by the arrow (blue in colour). The TSAW reflects from the cavity of size $2.6 \mathrm{~mm}$, which is at a distance less than the SAW attenuation length for a frequency of $15.8 \mathrm{MHz}(3.2 \mathrm{~mm})$. The sensing cavity filled with the fluid has a diameter of $2 \mathrm{~mm}$.

\subsubsection{Design and working principle of the sensing stage}

The sensing constitutes the last stage of the integrated device. It consists of two pairs of IDTs: one for the sensing the particles (indicated by IDTs-C in the Fig $5.1(a)$ ) and the other as a reference (denoted by IDTs-D). The reference cavity is filled with DI water (see symbol-E in Fig 5.1(a)). The signal from the active-IDT is calibrated with the reference-IDT to account for variation in the laser intensity and other substrate changes including temperature effects. The SAW-PA sensor consists of ten pairs of split-finger IDTs with a uniform electrode width and spacing of $49.75 \mu \mathrm{m}$ (Refer Fig 5.3 
for the s-parameters of the sensing IDTs). The first and the most important criteria for designing the SAW-PA sensor is in selecting the optimum working frequency. The details about the significance of choosing the correct frequency were discussed in our previous work [154]. According to the theory of photoacoustics (PA), the laser excitation on a spherical particle gives rise to a wideband bipolar pressure wave with the centre frequency $(f)$ provided by $[86,87]$.

$$
f=\frac{v_{s}}{2 R_{s}}
$$

where $v_{s}$ denotes the speed of sound in the polystyrene particle $\left(2350 \mathrm{~m} \mathrm{~s}^{-1}\right)$ [181] and $R_{s}$ is the radius of the particle. For a particle of size $10 \mu \mathrm{m}$ (diameter), the centre frequency is calculated to be $235 \mathrm{MHz}$. From PA theory, it appears that designing a SAW transducer at $235 \mathrm{MHz}$ is more sensitive. However, our previous work [154] signifies that the PA signal generated inside the microfluidic channel undergoes acoustic resonance and mode converts into a SAW. We performed a 2D numerical simulation using COMSOL Multiphysics 4.4 to determine the SAW-PA resonant frequencies (refer section 5.3.3.1 for details on the numerical simulation model). The frequency response study results revealed that the resonant frequencies were dominated at low frequencies. All frequencies above $50 \mathrm{MHz}$ were strongly attenuated, explaining that the PA centre frequency was down-converted to a much lower frequency by the acoustic resonance within the microfluidic channel. To further validate the accuracy of the numerical simulation model, we fabricated sensors with a centre frequency of 235and $10-\mathrm{MHz}$ and used it to sense an equal number of particles in the cavity (see Fig 5.5 for the transient SAW-PA signals from the sensors). The high-frequency SAW device (centre frequency of $235 \mathrm{MHz}$ ) consists of ten pairs of IDTs with an IDT-pitch of $8.46 \mu \mathrm{m}$ (diameter). The experimental results confirm that the SAW IDT at $10 \mathrm{MHz}$ was more sensitive than the $235 \mathrm{MHz}$ SAW IDT to detect the SAW-PA signals from the ten particles. The peak amplitude of the $10 \mathrm{MHz}$ device is ten times larger than the $235 \mathrm{MHz}$ SAW device. We developed a model to establish the relationship between 


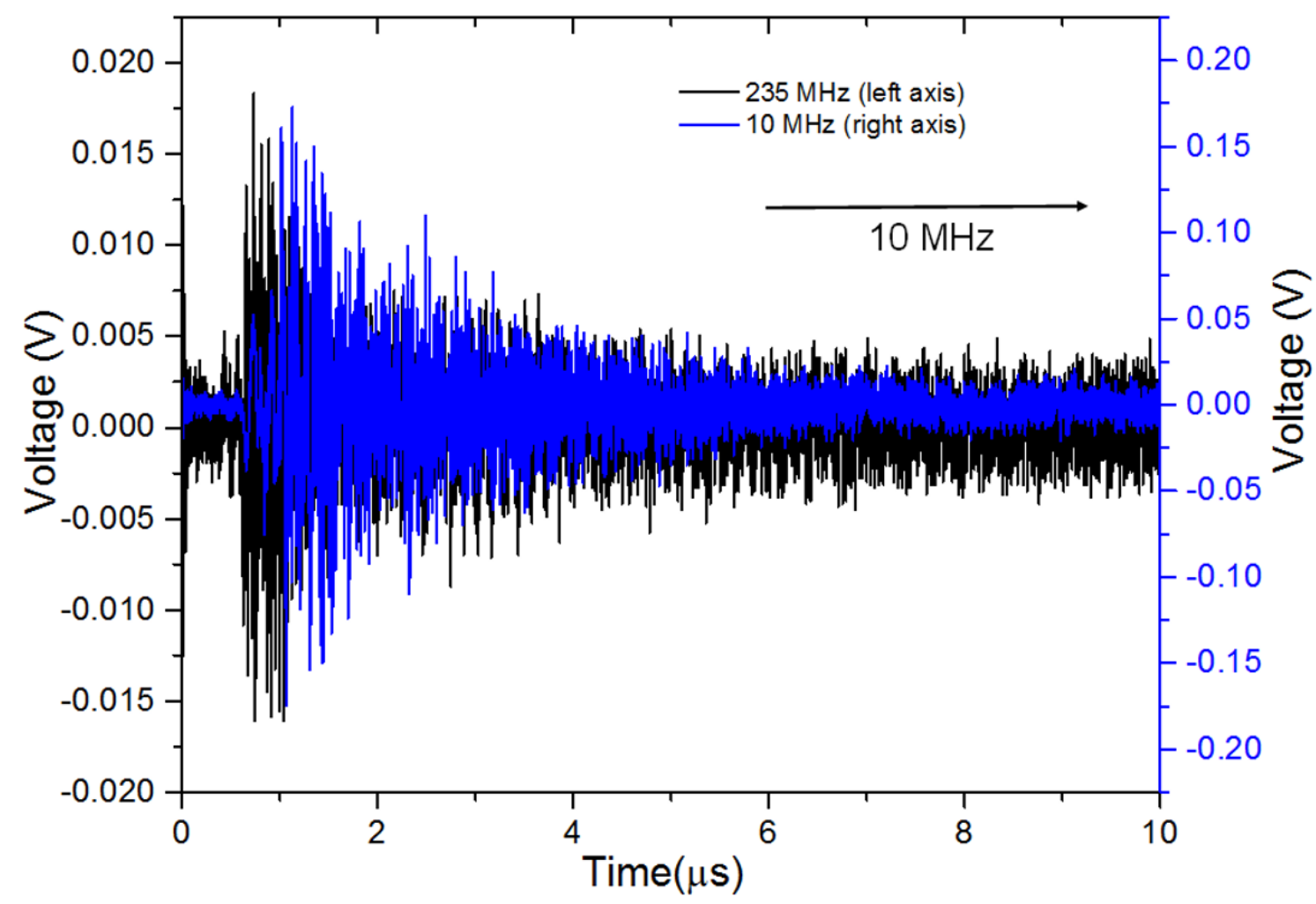

Figure 5.5: SAW-PA signals obtained from two SAW devices with a centre frequency of $10 \mathrm{MHz}$ and $235 \mathrm{MHz}$, for the same concentration of $10 \mu \mathrm{m}$ (diameter) particles.

the measured SAW-PA signal and the particle count. The SAW-PA amplitude can be expressed as:

$$
\begin{gathered}
A_{S A W-P A}=K_{S A W-P A}^{1} N_{p}+K_{S A W-P A}^{2}+n_{A} \\
K_{S A W-P A}^{1}=\Gamma_{p} \eta_{t h p} \mu_{a p} F K_{P A-S A W} K_{I D T} K_{a m p} \\
K_{S A W-P A}^{2}=\Gamma_{f} \eta_{t h F} \mu_{a F} F K_{P A-S A W} K_{I D T} K_{a m p}
\end{gathered}
$$

where $K_{S A W-P A}^{1}$ and $K_{S A W-P A}^{2}$ is a constant value, which denotes the SAW-PA output signal from a single particle and background (including the water and substrate) respectively. It includes the Grueneisen parameter $(\Gamma)$, heat conversion percentage $\left(\eta_{t h}\right)$, absorption cross-section $\left(\mu_{a}\right)$, optical fluence $(F)$, longitudinal wave generated by PA inside the fluid to surface acoustic wave conversion efficiency $\left(K_{P A-S A W}\right)$, acoustic 
to electrical conversion efficiency of the IDT $\left(K_{I D T}\right)$ and amplifier gain $\left(K_{a m p}\right)$. The subscript $p$ and $f$ denotes the particle and the fluid respectively. $N_{p}$ is the number of particles. The power spectral density (PSD) of a signal $y(t)$ can be expressed as:

$$
E\left\{y^{2}(t)\right\}=\frac{1}{2 \pi} \int_{-\infty}^{+\infty} S_{y y}(j \omega) d \omega
$$

where $E()$ is the mean and $S_{y y}(j \omega)$ is the power spectral density of $\mathrm{y}(\mathrm{t})$. Let the signal $\mathrm{y}(\mathrm{t})$ be represented as in (Eq. 5.2):

$$
\begin{aligned}
& E\left\{y^{2}(t)\right\}=( \\
&\left.K_{S A W-P A}^{1} N_{p}\right)^{2}+\left(K_{S A W-P A}^{2}\right)^{2}+ \\
& E\left(n_{A}^{2}\right)+2 K_{S A W-P A}^{1} N_{p} K_{S A W-P A}^{2}+2 K_{S A W-P A}^{1} N_{p} E\left(n_{A}\right) \\
& \quad+2 K_{S A W-P A}^{2} N_{p} E\left(n_{A}\right)
\end{aligned}
$$

where $n_{A}$ is the generated output noise.

$$
E\left\{y^{2}(t)\right\}=\left(K_{S A W-P A}^{1}\right)^{2} N_{p}^{2}+\left(2 K_{S A W-P A}^{1} K_{S A W-P A}^{2}\right) N_{p}+\left(K_{S A W-P A}^{2}\right)^{2}+E\left(n_{A}^{2}\right)
$$

From Eq. 5.5 and Eq. 5.7, the PSD has a quadratic relation with the number of particles $\left(\mathrm{N}_{\mathrm{p}}\right)$. The validity of the linear scaling of the SAW-PA amplitude with the number of particles $\left(\mathrm{N}_{\mathrm{p}}\right)$ exists only if the SAW-PA signals generated from each particle undergoes constructive addition. The source of the SAW signal originates from the PA generated longitudinal wave impinging on the substrate. We can consider each point on the substrate below the sensing cavity as a source of SAW excitation. If the particles are separated from the substrate at a vertical distance, $h_{p}$, the signals would undergo linear addition by particle count. Eq. 5.8 shows the distance at which a transition from near field to far field occurs.

$$
h_{p}>\frac{D^{2}}{4 \lambda}
$$


$D$ is the diameter of the source.Only the particles irradiated by the laser generates the SAW-PA signal. Hence we consider the maximum value of $D$ as the laser spot size. $\lambda$ denotes the acoustic wavelength $\left(=v_{s} / f_{0}\right), v_{s}$ is the sound speed in water and $f_{0}$ is the centre frequency of the SAW device. Satisfying Eq. 5.8 for the particle separation from the substrate ensures that the particles are located in the far-field. Hence, each particle would constructively add the signals at any point on the substrate. For a SAW IDT at centre frequency $10 \mathrm{MHz}\left(\mathrm{f}_{0}\right)$ and a laser spot size of $200 \mu \mathrm{m}(D)$, the transition distance equals $68 \mu \mathrm{m}$. Due to the SAW concentration effects, the particles are aggregated at a height $\left(h_{p}\right)$ near the fluid-air interface inside the sensing cavity $(\sim 2 \mathrm{~mm})$. The concentration ensures that the condition mentioned in Eq. 5.8 is satisfied. The PA signals generated by the particles residing in the far-field will, therefore, undergo constructive addition. The linear scaling of the SAW-PA signal amplitude with increasing number of particles described in Eq. 5.2 is, therefore, valid

\subsubsection{Materials}

Ten- and three-micron diameter black coloured polystyrene beads (polyscience), with a concentration of $\sim 4.55 \times 10^{7}$ and $1.68 \times 10^{9}$ particles $/ \mathrm{mL}(2.5 \%$ solids-suspension) were used to characterise the SAW integrated platform. The black dye has a broad absorption spectrum across the visible light wavelength, with an absorbance value of $\sim 0.7$.

\subsection{Results and discussion}

\subsubsection{Separation of particles}

The taSSAW approach demonstrated separation efficiency of $100 \%$ and > $95 \%$ for the $10-$ and $3-\mu \mathrm{m}$ beads respectively. The separation of particles can be traced from the stacked images in Fig 5.6. The large particles $(10 \mu \mathrm{m})$ were collected 
in the upper outlet cavity (sensing reservoir), while the flow transported the smaller particle $(3 \mu \mathrm{m})$ to the lower outlet (waste reservoir). The ratio of the flow rate through the inlets was maintained in the ratio of 10:1:2. The upper inlet had the largest flow rate to prevent particles from flowing into the sensing outlet without turning on the SAW. The particle flow through the centre inlet has the lowest rate. The flow-rates
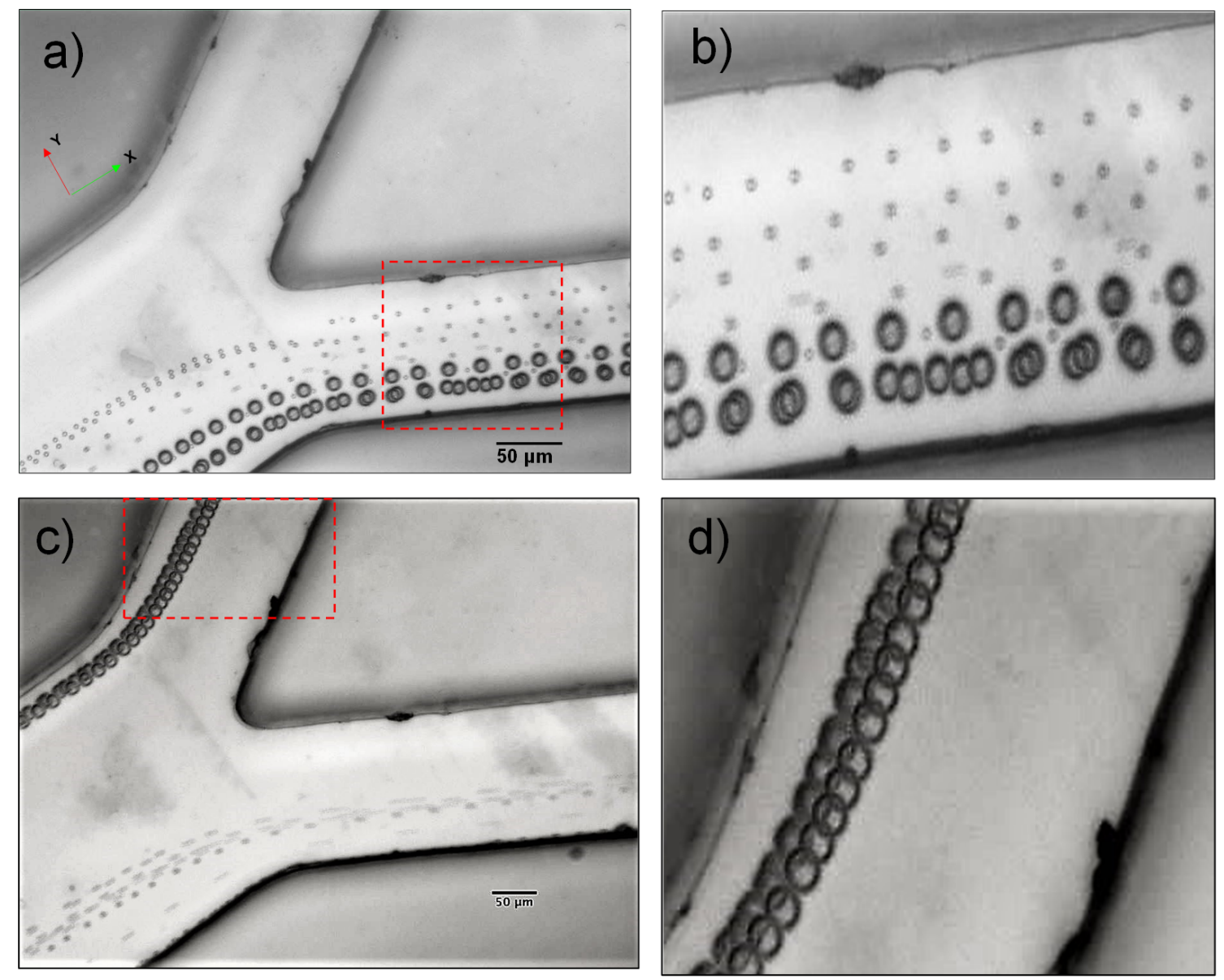

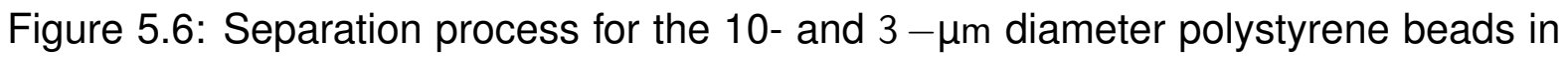
the outlet region of the microfluidic channel. (a) Shows the particle flowing to the waste reservoir when the SAW is turned OFF and (d) when the SAW is turned ON, the $10 \mu \mathrm{m}$ particles are pushed to the sensing outlet by the SAW acoustic radiation force.(b and d) shows the zoomed-in view of the waste outlet and the sensing outlet, respectively.

ensured that the particle flow was sandwiched between the two outer sheath flows, closer towards the bottom outlet. It was observed that increasing the power above 
$25 \mathrm{dBm}$ would push the $10 \mu \mathrm{m}$ particles into the upper part of the main channel and remain trapped. On turning off the SAW input, the trapped particles will continue with the flow into the sensing outlet. Further optimisations on the flow rate and the input SAW could achieve $100 \%$ separation efficiency for both the particle sizes.

\subsubsection{Concentration of particles}

The syringe pump was stopped when the separated particles filled the upper outlet. The cavity was filled to a volume of approximately $10 \mu \mathrm{L}$ in less than 10 mins. Fig 5.7 presents screen shots of the $10 \mu \mathrm{m}$ particles before and after the SAW exposure. As shown in Fig 5.7(a), particles are randomly positioned inside the cavity, even at various heights, before the SAW exposure. By exposing the suspension of $10 \mu \mathrm{m}$ particles in water to a $15.8 \mathrm{MHz}$ SAW at input amplitude of $26 \mathrm{dBm}$, the particles tend to aggregate, though not at the centre. The condition $K<1$ and $x_{s}>r_{d}$ (where $r_{d}$ in this case denotes one-half the sum of the cavity diameter and the width of the PDMS, $1.3 \mathrm{~mm}$ and $x_{s}=3.2 \mathrm{~mm}$ ) are satisfied in our design. According to the theory developed by Destgeer et al. [177], a droplet with particle satisfying the above two conditions will cause the particles to concentrate in the centre of the droplet. The shift in the location of the concentrated particles from the centre of the cavity could be due to the acoustic streaming force (ASF) pattern generated inside a PDMS cavity being different from a sessile droplet. Fig $5.7(\mathrm{~b}-\mathrm{d})$ shows that a small $(<10)$ to large $(200)$ number of particles are concentrated using the SAW. Image analysis software (ImageJ, NIH) was used to count the larger number of particles $(>100)$. A SAW exposure causes the particles to aggregate closer to the periphery of the droplet. The concentration process was relatively slow compared to the time taken for aggregation within a sessile droplet (few sec [177]). The concentration-time for an input power of $26 \mathrm{dBm}$ was 40-50 secs. The longer response time can be attributed to the weaker response of the IDT at the operating frequency (see Fig 5.3 for the $S 11$, which is $-4 \mathrm{~dB}$ ). For the subsequent sensing stage, the concentration attained is sufficient. The aggregation 

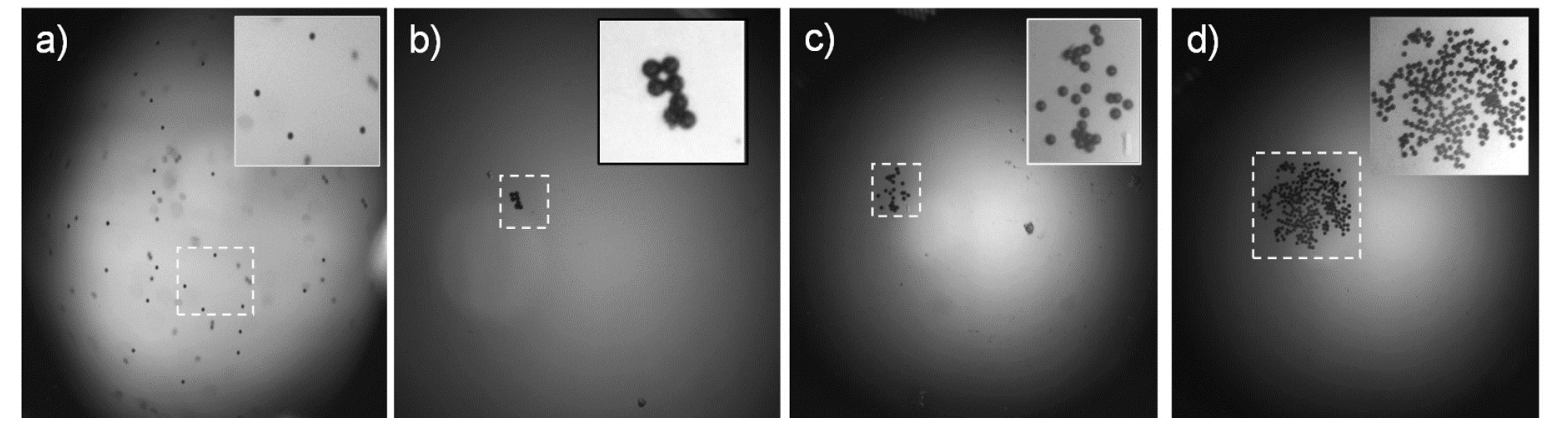

Figure 5.7: Ten micron (diameter) particles at different levels of concentration inside the sensing cavity. Screenshots of the particle-laden open-cavity before (a) and after (b-d) SAW exposure for a sufficient period to obtain the particles concentration at the required location.
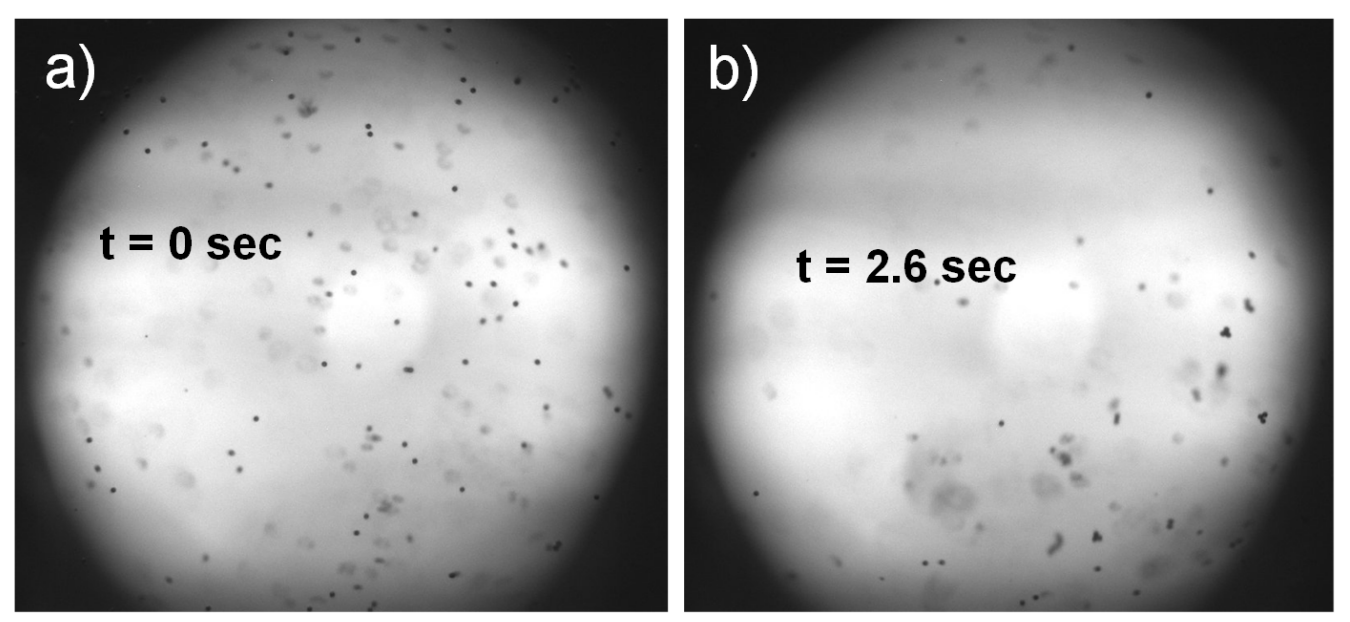

Figure 5.8: Particles of size $10 \mu \mathrm{m}$ placed inside an open cavity (surrounded by PDMS) of size $5 \mathrm{~mm}$ exposed to a $15.8 \mathrm{MHz}$ SAW. Particles do not concentrate, unlike a smaller sized cavity $(2 \mathrm{~mm})$ as shown in the figure before $(\mathrm{t}=0 \mathrm{~s})$ and after $(\mathrm{t}=2.6 \mathrm{~s})$ SAW exposure

helps to satisfy the two condition required: 1 ) the particles has to remain at a far-field distance. The particle aggregates at a distance closer to the free surface $(\sim 2 \mathrm{~mm})$. 2) The mechanical scanner operates at $1 \mathrm{~Hz}$, the response time of the sensing stage can be minimised by reducing the scanning area. Particles are concentrated within half of the cavity as shown in Fig $5.7(b-d)$. During the measurement, only half of the 
cavity containing the particles are scanned. While concentration is achieved using a $10 \mu \mathrm{m}$ particle inside a cavity of diameter $2 \mathrm{~mm}$, the particles fail to aggregate within the cavity of $5 \mathrm{~mm}$ diameter. The particles circulate under the acoustic streaming forces inside the fluid. The particle arrangement before and after SAW exposure in a $5 \mathrm{~mm}$ sized cavity is shown in Fig 5.8. SAW exposure at $15.8 \mathrm{MHz}$ for $2.6 \mathrm{~s}$ at $26 \mathrm{dBm}$ causes streaming inside the cavity, but the particles do not aggregate. The attenuation length at $15.8 \mathrm{MHz}$ is smaller than the dimension of the cavity, and as a result, there is no standing wave generated to create an aggregation of particles. Optimisation of the SAW frequency and cavity dimensions will reduce the concentration-time further.

\subsubsection{Sensing of particles}

\subsubsection{Numerical simulation}

Two-dimensional (2-D) frequency domain simulation is performed using COMSOL Multiphysics. The numerical simulation is carried out with two objectives: (a) To investigate the relationship between the number of particles $\left(N_{p}\right)$ and the amplitude of the piezoelectric surface displacement. (b) Study the SAW-PA frequency response of the particle to design the IDT for the sensor. The SAW concentration stage aggregates the particles in a horizontal 2-D plane (X-Z plane) at a certain height $\left(h_{t}\right)$ from the substrate. We utilise this functionality to simplify the numerical model. In the simulation, particles are arranged along the X-direction (see Fig 5.9 for the axis direction) in a linear array. We assume that the particles arranged in the Z-direction focused at a far-field distance from the substrate will linearly contribute to the acoustic displacements with $\mathrm{Np}$. The assumptions simplify the simulation to a 2-D model $(X-Y$ plane). Fig 5.9 shows the simulation geometry. It consists of a PDMS microfluidic channel with an open-cavity of width $w_{1}=2 \mathrm{~mm}$ and height $h_{1}=3 \mathrm{~mm}$. The PDMS channel is trimmed at the bottom with a thickness $w_{2}=300 \mu \mathrm{m}$. The trimming ensures that the cavity length for concentrating the particles as shown in Fig 5.4 is $2.6 \mathrm{~mm}$. 
The PDMS at the upper section extends for a longer length. The infinite domain of the PDMS is modelled using a perfectly matched layer (PML) which causes the wave to pass through without reflection from the sides. The PML is represented at the ends of the PDMS and piezoelectric domain. The polystyrene particles are arranged in a linear array at a height $h_{t}=2 \mathrm{~mm}$ from the substrate surface.

Thermoacoustics, Frequency domain physics was used to solve the pressure generated by the input heat from the polystyrene particles. Each polystyrene particle represented a heat source of $1 \mathrm{~W} / \mathrm{m}^{3}$. The top boundary of the water filled domain is modelled as a wall with normal impedance boundary condition and adiabatic thermal condition. The impedance offered by air is set to $420 \mathrm{~Pa} \mathrm{~s} / \mathrm{m} \mathrm{[182].} \mathrm{The} \mathrm{pressure}$ from the thermoacoustic domain is coupled to the pressure in the PDMS through the thermoacoustic-pressure acoustics boundary condition. Further, the pressure is coupled to the piezoelectric domain by the Multiphysics coupling nodes of the thermoacoustic/pressure acoustics- structure interaction. A soft boundary condition is used at the PDMS boundary to model the PDMS-air boundary. Fig 5.10(a) gives the result of the frequency domain simulation. The colour plots in the PDMS/fluid and piezoelectric domain shows the pressure and acoustic displacement respectively at $10 \mathrm{MHz}$ for 10 particles arranged at a height $\left(h_{t}\right)$ of $2 \mathrm{~mm}$ (in Y-direction). The fluid is filled in a cavity of height $3 \mathrm{~mm}$ which is matched to the cavity dimension used in the experiment. In the simulation, the particles are arranged linearly in the X-direction at a height $h_{t}=2 \mathrm{~mm}$. Fig 5.10(b) shows the frequency response of the acoustic displacement at a point (close to the IDT) on the $\mathrm{LiNbO}_{3}$ substrate. The magnitude of the acoustic displacements drops for frequencies above $50 \mathrm{MHz}$, which could be attributed to the mechanical resonances inside the PDMS cavity that attenuates the high-frequency modes. The acoustic displacement has the largest magnitude at the $9 \mathrm{MHz}$ frequency. Further, the effect of particle count $\left(N_{p}\right)$ on the SAW displacement was studied. The sum of the frequency response from $9-11 \mathrm{MHz}$ is calculated as the sensing parameter, as it matches with the bandwidth of the IDT designed for the sensor. Fig 5.10(c) shows 


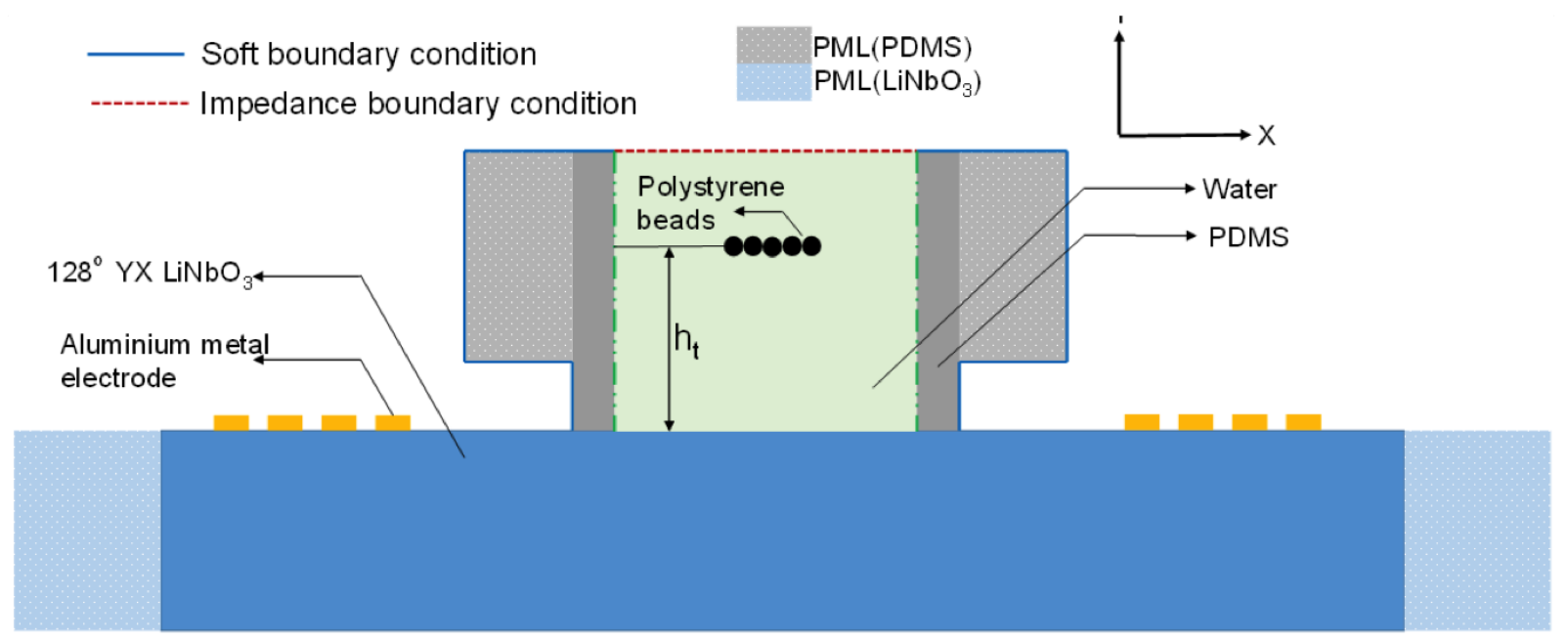

Figure 5.9: 2D Geometry used for the simulation with the corresponding domains. The fluid channel of width $w_{1}=2 \mathrm{~mm}$ and height $h_{1}=3 \mathrm{~mm}$ used for the simulation. It consists of a PDMS chip (grey), water (green) with polystyrene beads (black), piezoelectric transducer (blue, Lithium niobate, $\mathrm{LiNbO}_{3}$ ) surrounded by air. The polystyrene beads are placed at a height, $h_{t}(2 \mathrm{~mm})$ from the surface of the piezoelectric material. Perfectly matched layers (PML) are placed at the ends of the piezoelectric and PDMS domains to avoid reflections from the ends. An impedance boundary condition (red dotted line) at the water-air interface and soft boundary condition at the PDMS-air interface (continuous blue line) represents the boundaries in the model. The figure is not drawn to scale.

that a linear relationship exists between $\mathrm{N}_{\mathrm{p}}$ and the sensing parameter. From Eq. $5.2 \mathrm{a}$ linear relationship between particle count and SAW displacement is expected, which aligns the theory and the numerical simulation.

\subsubsection{Experimental results}

The laser beam of spot size $200 \mu \mathrm{m}$ was focussed into the concentrated particles for sensing. The alignment of the laser focus into the particles was achieved by mechanical scanning. The scanner was moved in the horizontal plane at a step size of $50 \mu \mathrm{m}$. At each scanning point, the pulsed laser at the repetition rate of $10 \mathrm{~Hz}$ was irradiated. Signals were averaged for ten cycles. After SAW actuation, a concentration of 1-200 particles per $10 \mu \mathrm{L}$ of the solution was aggregated within one-half of the PDMS 

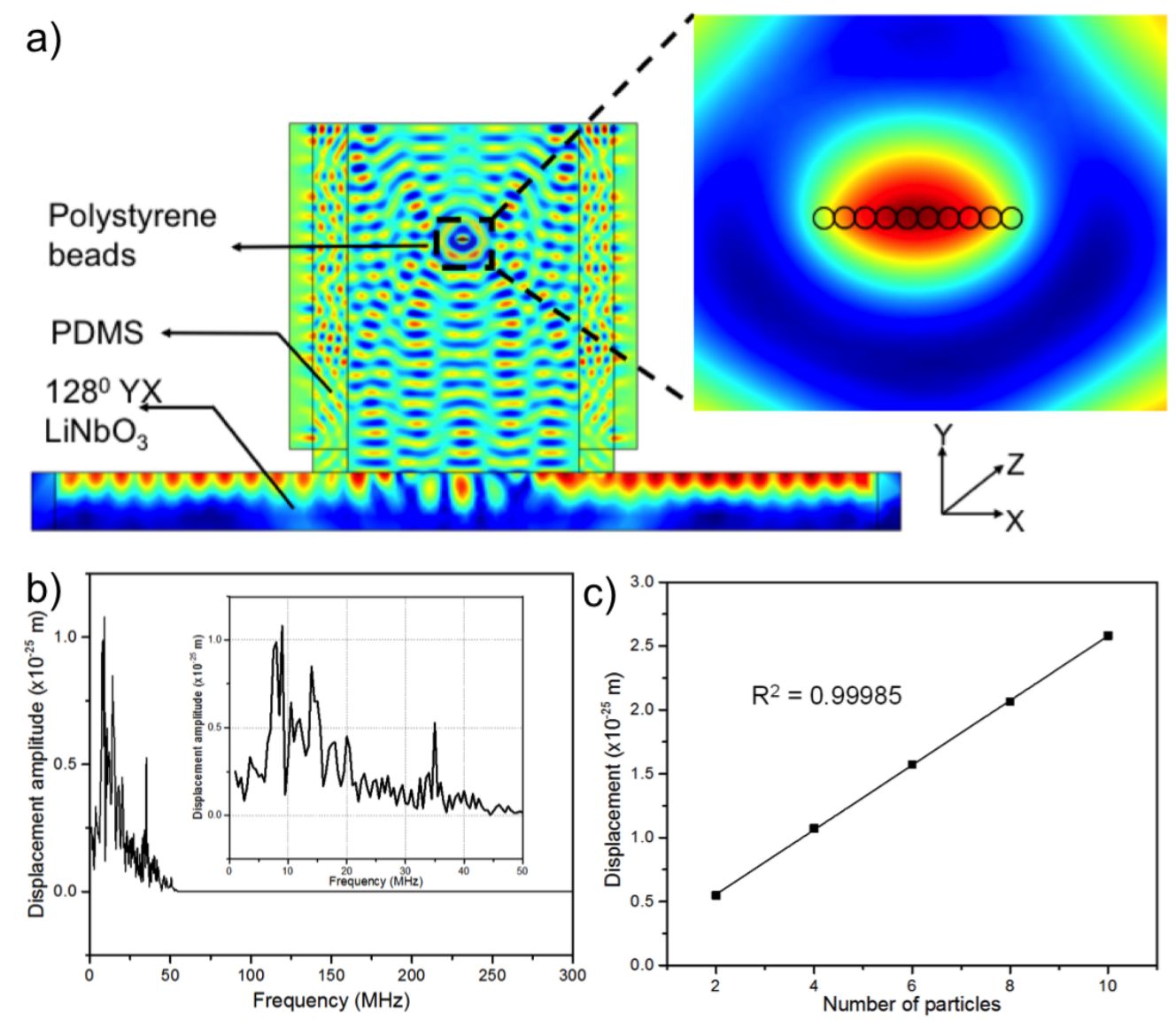

Figure 5.10: Numerical simulation results of particle detection using photoacoustic induced surface acoustic wave. (a) Colour plots of the 2-D simulation illustrating the acoustic pressure in the water-PDMS domain and y-axis displacement in the $\mathrm{LiNbO}_{3}$ substrate, at $9 \mathrm{MHz}$ for ten particles located at a height $\left(h_{t}\right)$ of $1 \mathrm{~mm}$. (b) The frequency response of the displacement amplitude at a point on the surface of the substrate from $1 \mathrm{MHz}-300 \mathrm{MHz}$, with peak amplitude at $9 \mathrm{MHz}$. The inset shows zoomed in frequency response from $1 \mathrm{MHz}-50 \mathrm{MHz}$. (c) Linear response of the acoustic displacement to the number of particles $(\mathrm{R} 2=0.99985)$.

cavity. Hence, the scanning area is limited to half of the cavity size, equal to an area of $1500 \mu \mathrm{m} \times 750 \mu \mathrm{m}$, with 450 scanning points. The total response time is the sum of the scan time and signal averaging time. For a mechanical scanner of rate $1 \mathrm{~Hz}$ and signal averaging time of $1 \mathrm{sec}$ for each point, the total response time is $15 \mathrm{mins}$. To demonstrate quantitative measurement of particle counts, we prepared solutions with different concentrations. A $10 \mu \mathrm{m}$ diameter particle with a stock concentration of 
$\sim 4.55 \times 10^{7}$ particles per millilitre $(2.5 \%$ solids-suspension $)$ was selected. The solution was diluted to prepare an initial concentration of $\sim 200$ particles per $10 \mu \mathrm{L}$. The solution was further serial diluted by two times to obtain concentrations of $100,50,25$, and 10 particles per $10 \mu \mathrm{L}$.

\subsubsection{Signal processing}

The use of reference SAW calibrates the variation of laser energy and other substrate variations. The reference SAW alleviates the requirement of a photodiode to calibrate fluence variation. The signal obtained from the sensing SAW is normalised by the variations in the reference SAW signal. The SAW sensor IDT is designed with a centre frequency of $10 \mathrm{MHz}$ with a bandwidth of $10 \%$. The sum of the power spectral density of the signal from $9-11 \mathrm{MHz}$ is selected as the sensing parameter. The sensed parameter obtained from each scanned point is interpolated using MATLAB interpolation function (interp2). The interpolation is used to get a continuous range of values of the sensing parameter from the spatially discrete signals obtained from the mechanical scanning. The maximum value obtained from the interpolation function is selected as the quantitative metric for various concentrations. The scanning step size of $50 \mu \mathrm{m}$ for a laser spot size of $200 \mu \mathrm{m}$ satisfies the Nyquist sampling rate condition for interpolation. Fig 5.11 shows the experimental results for different particle counts. Fig 5.11 (a) shows the transient waveform obtained from a single IDT for $10 \mu \mathrm{m}$ particles at a concentration of c.a 155 particles per $10 \mu \mathrm{L}$. The voltage waveforms were captured at the two sensing IDTs (IDT1 and IDT2). The sensor demonstrated a quadratic response $\left(R^{2}=0.98\right)$ between the sum of power spectral density (PSD) in the 9-11 $\mathrm{MHz}$ (SAW-PA sensing parameter) and the particle concentration (varying from $\sim 7$ 200 particles per $10 \mu \mathrm{L}$ range). The sensing parameter was normalised by the PSD calculated from the reference cavity. Hence, the variations in temperature and laser fluence are calibrated. The experimental response fits the analytical model presented in Eq. 5.7. The target fitting function was $Y=K_{1} N_{p}^{2}+K_{2} N_{p}+K_{3}$, where $K_{1}, K_{2}$ and $K_{3}$ 

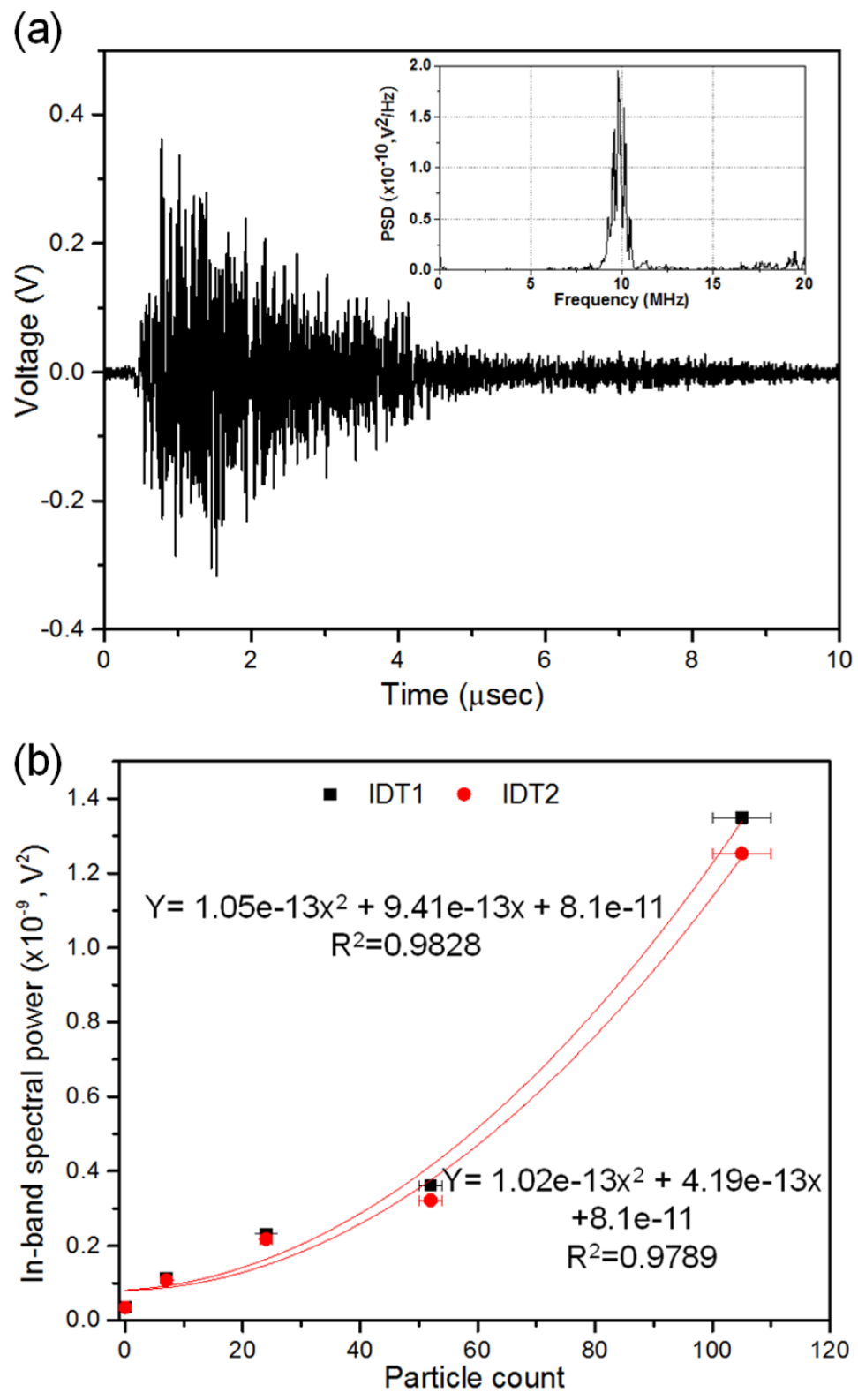

Figure 5.11: SAW-PA signal from $10 \mu \mathrm{m}$ (diameter) polystyrene beads at different concentrations. (a) Voltage waveform for $\sim 155$ particles in $10 \mu \mathrm{L}$ detected using the SAW-PA sensor. The inset shows the corresponding power spectral density. (b) Inband spectral power $(9-11 \mathrm{MHz})$ for various particle counts at the two IDTs. The spectral power followed a second-order polynomial $Y=K_{1} N_{p}^{2}+K_{2} N_{p}+K_{3}$, with $R^{2}$ value of 0.9828 and 0.9789 for IDT1 and IDT2 respectively. 
are unknown constants. The actual particle count was obtained from the CCD camera images: by manually counting for particles at low concentration and using particle counting functionality in ImageJ for larger concentrations ( 200 particles). For particles at the larger concentration shown in Fig 5.7(d), the concentration area was bigger than the spot size of $200 \mu \mathrm{m}$. Fitting the output signal to the fitting curve shown in Fig 5.11 , we obtain a particle count equal $\sim 155(+/-3)$, where the deviation is obtained at IDT1 and IDT2, respectively. The calculated value was less than the total particle count of 194 (using ImageJ). The maximum number of particles fitting a circle of size $200 \mu \mathrm{m}$ (using least square fitting) was found to be c.a 152 which is close to the particle count obtained by fitting the SAW-PA sensing parameter to the quadratic response curve. The response time of the sensor is limited currently by the mechanical scanner $(1 \mathrm{~Hz})$ and laser pulse repetition rate $(10 \mathrm{~Hz})$. An optical scanning mirror (in $\mathrm{KHz}$, Thorlabs) and laser with higher repetition rate $(100 \mathrm{~Hz})$ can reduce the response time to less than a minute.

\subsection{Conclusion}

We have developed a SAW-PA integrated microfluidic system that combined standing surface acoustic wave based separation, particle concentration and sensing on a single $\mathrm{LiNbO}_{3}$ substrate and demonstrated real-time quantitative detection of polystyrene beads. Though SSAW methods can achieve high separation efficiency ( $\sim 100 \%)$, the downstream detection is currently performed off-chip cell counters and haemocytometers, which risks the possibility of sample loss while transferring. We demonstrate that a pulsed laser illuminating the separated particles can generate a photoacoustic induced SAW (SAW-PA), which can be analysed to perform a label-free quantitative detection of the sample in a few mins with a sensitivity of $\sim 7-200$ particles per $10 \mu \mathrm{L}$ of the sample solution. The system can be extended to label free detection of cells from whole blood and other body fluids such as urine. A single cell level 
sensitivity is possible by using optimising the SAW design and microfluidic channel dimensions to match the acoustic resonance inside the microfluidic channel. 


\section{Chapter 6}

\section{Conclusions and Future Work}

In this chapter, we provide a summary of the work carried in this thesis and outline the significant contributions made. The chapter and dissertation will conclude with potential future works that could arise from these findings.

\subsection{Conclusions}

In this thesis, we commenced with an overview of lab-on-a-chip (LOC) systems and discussed an important microfluidic platform based on surface acoustic wave (SAW) devices. The capability to perform actuation and sensing makes the SAW device a potential candidate for realising an LOC. To explore the field of SAW in microfluidics, we first looked into the use of SAW as an actuator, where we conducted a comprehensive experimental and theoretical work related to the acoustic energy transmission to a fluid in a reusable microfluidic channel. Followingly, we proposed and demonstrated using extensive experimental and theoretical study, a novel sensor for characterising optical and acoustical properties of dyes and nanoparticles in a microfluidic channel. The sensor relies on detecting the SAW generated by photoa- 
coustics (PA) in a microchannel. Later, by performing a detailed numerical modelling using finite element method (FEM) we unravelled the existence of optically induced acoustic resonance inside the microfluidic channel and efficient mode conversion of a longitudinal wave into SAW at certain frequencies dependent on the microchannels dimensions. Finally, we demonstrate an integrated microfluidic platform, which combines the microfluidic actuation (separation, concentration) and sensing (detection of particles) on a single lithium niobate substrate. The main contribution of this thesis are as follows:

(i) Compared to other areas studied on SAW, less research effort has been concentrated on using a disposable superstrate for SAW microfluidic applications. The first complete study of SAW coupling to fluids in the microfluidic channel on disposable superstrate was revealed. We studied the influence of input voltage, frequency and coupling layer thickness on the acoustic energy induced mixing efficiency. The main significance of this work comes from the development of analytical models which can be used to optimise the acoustic energy transmission to the fluid and improve the mixing efficiency.

(ii) We propose for the first time a SAW-PA device which detects the PA generated in a microfluidic channel with a SAW device. The existence of optoacoustic resonance inside the microfluidic channel converts the wideband PA into a narrow band signal, justifying the reason for using SAW (high-Q) as a sensitive detector. This label-free sensing platform facilitates the characterization of liquid samples by measuring both it's optical and mechanical properties simultaneously. Furthermore, the usage of SAW as the receiver of the PA signal in this new design enables a couplant-free (water-free) PA signal sensing in a low cost and compact pathway.

(iii) We propose a SAW-PA integrated microfluidic platform which can perform a label-free quantitative detection of the separated particles on the same piezo- 
electric substrate. The sensor exhibited detection of the $10 \mu \mathrm{m}$ particles down to 7 particles in $10 \mu \mathrm{L}$ of the sample volume in 15 minutes. To the best of our knowledge, this is for the first time that a fully integrated microfluidic system using SAW is exhibited.

\subsection{Future work}

(i) Application of the integrated platform for detecting rare cells.

Though separation of rare circulating tumour cells was performed using a taSSAW device, its applicability as a diagnostic device is limited due to the lack of an integrated downstream detection. In this scenario, the SAW-PA integrated microfluidic system is an excellent choice. This work requires optimisation of sensor to detect down to a single cell, as the rare CTCs are present in a concentration of $\sim 100$ cells $/ \mathrm{mL}$. Further, to achieve the throughput (process one $\mathrm{mL}$ of sample) for rare CTC detection, requires lowering the sensors response time to $\sim 1 \mathrm{~min}$ for $10 \mu \mathrm{L}$ of solution. The various possibilities could include; using optical scanning mirrors, high pulse repetition rate or by precisely controlling the position of cells in the channel using SAW based tweezers, which can even discard the use of scanning system.

(ii) Portable SAW-PA sensor:

The portability of the SAW-PA sensor is limited due to the requirement of having a bulky solid-state pulsed laser. In this work, we are exploring the use of laser diodes which are relatively simple, compact and inexpensive. However, the limitation with laser diodes is its limited pulse energy (for nanosecond time duration) of several microjoules. The sensitivity of the SAW device has to be improved to detect such weak PA-induced SAW signals.

(iii) Low concentration detection of analytes. 
In Section 4.4.4 we calculated the sensitivity of the SAW-PA sensor to detect a squaraine dye to $508 \mu \mathrm{V} / \mathrm{nM}$. In this work, we could explore the limit of detection of the SAW-PA sensor. The lower response time $(<1 \mathrm{~min})$ owing to lack of surface treatment and biofunctionalization along with high sensitivity (nM) could pave wave to state-of-art performance improvement [76]. 


\section{Appendix A}

\section{Lamb waves in a plate}

Here we derive an equation for the displacement of a lamb wave excited in an isotropic plate shown in Fig A.1, following the derivation provided in [132].

The displacement of a material can be considered to be the sum of a scalar $\phi$ and vector potentials $\psi$, given by

$$
u=\nabla \phi+\nabla \times \psi
$$

where the component $\nabla \phi$ and $\nabla \times \psi$ represents the longitudinal and the transverse components of the displacements. From vector identities, the curl of a gradient of a

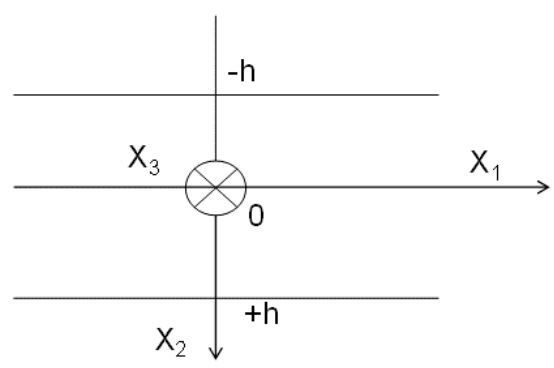

Figure A.1: Isotropic Plate 
scalar field $\nabla \times \nabla \phi$ and the divergence of the curl of any vector field $\nabla . \nabla \times \psi$ is zero. Hence, $\nabla \phi$ should act in the direction of the wave propagation, forming a longitudinal wave. While, $\nabla \times \psi$ should act orthogonal to the propagation direction constiuting a transverse wave. The potentials satisfy the following wave equations.

$$
\begin{aligned}
& \nabla^{2} \phi-\frac{1}{V_{L}^{2}} \frac{\partial^{2} \phi}{\partial t^{2}}=0 \\
& \nabla^{2} \psi-\frac{1}{V_{T}^{2}} \frac{\partial^{2} \psi}{\partial t^{2}}=0
\end{aligned}
$$

where $V_{L}$ and $V_{T}$ denotes the phase velocities of the longitudinal and transverse waves respectively. The lamb wave is considered to be propagating in the $x_{1}$ direction, and considering there is no diffraction or variation along the direction of $x_{3}$, with $\partial_{3} \equiv \frac{\partial}{\partial x_{3}}=$ 0 . For the sinusoidal propagation, $\frac{\partial}{\partial x_{1}}=\partial_{1}=-i k$. From Eq. A.1, we can write the displacement components as:

$$
\begin{gathered}
u_{1}=\partial_{1} \phi+\partial_{2} \psi_{3}=-i k \phi+\partial_{2} \psi_{3} \\
u_{2}=\partial_{2} \phi-\partial_{1} \psi_{3}=\partial_{2} \phi+i k \psi_{3}
\end{gathered}
$$

Defining $p$ and $q$ as

$$
\begin{aligned}
& p^{2}=\frac{\omega^{2}}{V_{L}^{2}}-k^{2} \\
& q^{2}=\frac{\omega^{2}}{V_{T}^{2}}-k^{2}
\end{aligned}
$$

The Laplacian operator is given by:

$$
\nabla^{2}=\frac{\partial^{2}}{\partial x_{1}^{2}}+\frac{\partial^{2}}{\partial x_{2}^{2}}=-k^{2}+\frac{\partial^{2}}{\partial x_{2}^{2}}
$$

Using Eq. A.2, Eq. A.4 and Eq. A.5, the functions $\phi$ and $\psi \equiv \psi_{3}$, satisfies the eqn:

$$
\begin{aligned}
\delta_{2}^{2} \phi+p^{2} \phi & =0 \\
\delta_{2}^{2} \psi+q^{2} \psi & =0
\end{aligned}
$$




\section{A.1 Derivation of stress in an isotropic solid}

For an isotropic solid, the elastic tensor $c_{i j k l}$ can be represented by two independent constants referred to as Lame constants $\lambda$ and $\mu$ by the following equation:

$$
c_{i j k l}=\lambda \delta_{i j} \delta_{k l}+\mu\left(\delta_{i k} \delta_{j l}+\delta_{i l} \delta_{j k}\right)
$$

Since the tensors $T_{i j}$ and $S_{k l}$ representing the stress and strain tensors are symmetric, the number of independent elastic constants reduces from 81 to 36 . Assigning value of 1 to 6 for the pairs (ij) and (kl), we get the following relations:

$$
\begin{array}{r}
c_{11}=c_{22}=c_{33}=\lambda+2 \mu \\
c_{12}=c_{23}=c_{13}=\lambda \\
c_{44}=c_{55}=c_{66}=\mu=\left(c_{11}-c_{12}\right) / 2
\end{array}
$$

Using Hooke's law in an isotropic solid, the normal stress $\left(T_{11}, T_{22}, T_{33}\right)$ can be written as:

$$
T_{i i}=c_{i i k l} S_{k l}
$$

substituting Eq. A.7 in Eq. A.9 with $j=i$, we get

$$
T_{i i}=\lambda\left(S_{11}+S_{22}+S_{33}\right)+2 \mu S_{i i}
$$

and the tangential stress $T_{i j}$, with $i \neq j$ is given by:

$$
\begin{array}{r}
T_{i j}=\mu\left(\delta_{i k} \delta_{j l}+\delta_{i l} \delta_{j k}\right) S_{k l} \\
T_{i j}=2 \mu S_{i j}, i \neq j
\end{array}
$$

From Eq. A.12 and Eq. A.11, we can define the stress tensor as:

$$
T_{i j}=\lambda S \delta_{i j}+2 \mu S_{i j}
$$


where $S=S_{11}+S_{22}+S_{33}$ is the dilation. Since $S_{33}=0$, the dilation is :

$$
S=S_{11}+S_{22}=\delta_{1} u_{1}+\delta_{2} u_{2}=\nabla^{2} \phi
$$

\section{A.2 Derivation of mechanical displacement components of Lamb wave}

The solution of Eq. A.6 should satisfy the stress free conditions, hence $T_{22}=$ $T_{2}=0$ and $T_{12}=T_{6}=0$ at $x_{2}= \pm h$. The normal stress $T_{2}=T_{22}$ is defined by using equation Eq. A.12 and first of Eq. A.8.

$$
\begin{array}{r}
T_{2}=T_{22}=c_{11} S-2 c_{66} S_{11} \\
=c_{11} \nabla^{2} \phi-2 c_{66} \partial_{1} u_{1} \\
T_{2}=c_{11} \nabla^{2} \phi+2 c_{66}\left[k^{2} \phi+i k \partial_{2} \psi\right]
\end{array}
$$

The last step of Eq. A.14 follows from Eq. A.3. Using Eq. A.2, Eq. A.5, Eq. A.6, we find

$$
\rho \omega^{2}=\left(k^{2}+q^{2}\right) c_{66}
$$

From Eq. A.2:

$$
c_{11} \nabla^{2} \phi=-\rho \omega^{2} \phi
$$

Substituting Eq. A.16 in Eq. A.14

$$
T_{2}=-\rho \omega^{2} \phi+2 c_{66}\left[k^{2} \phi+i k \partial_{2} \psi\right]
$$

Further using Eq. A.15 in Eq. A.17,

$$
T_{2}=c_{66}\left[\left(k^{2}-q^{2}\right) \phi+2 i k \partial_{2} \psi\right]
$$


Next we define the tangential stress $T_{6}=T_{12}$. From Eq. A.11,

$$
\begin{array}{r}
T_{6}=2 c_{66} S_{12} \\
=c_{6} 6\left[\partial_{2} u_{1}+\partial_{1} u_{2}\right]=c_{6} 6\left[\partial_{2}^{2} \psi-2 i k \partial_{2} \phi+k^{2} \psi\right]
\end{array}
$$

Using Eq. A.6 in Eq. A.19, we get

$$
T_{6}=c_{66}\left[\left(k^{2}-q^{2}\right) \psi-2 i k \partial_{2} \phi\right]
$$

The stress free conditions $T_{2}=T_{6}=0$ at $x_{2}= \pm h$ are satisfied only if the stresses $T_{2}$ and $T_{6}$ are even or odd functions of $x_{2}$. Hence, we define the solution for the potential functions as:

$$
\begin{aligned}
& \phi=B \cos \left(p x_{2}+\alpha\right) \\
& \psi=A \sin \left(q x_{2}+\alpha\right)
\end{aligned}
$$

Using Eq. A.21 in Eq. A.3, gives the mechanical displacements

$$
\begin{aligned}
& u_{1}=-i k B \cos \left(p x_{2}+\alpha\right)+q A \cos \left(q x_{2}+\alpha\right) \\
& u_{2}=-p B \sin \left(p x_{2}+\alpha\right)+i k A \sin \left(q x_{2}+\alpha\right)
\end{aligned}
$$

From Eq. A.22, we note that there are two types of lamb waves corresponding to:

(i) Symmetric mode for $\alpha=0$, here we note from Eq. A.22 that the longitudinal component $u_{1}$ is an even function of $x_{2}$, while the transverse component $u_{2}$ is an odd function.

(ii) Antisymmetric mode for $\alpha=\pi / 2$, where the longitudinal and the transverse components are odd and even functions of $x_{2}$ respectively. 
Next we derive the boundary conditions $T_{2}=T_{6}=0$ on $x_{2}=h$. Using Eq. A.21 on Eq. A.18 and Eq. A.20 results in the following equation.

$$
\begin{aligned}
& \left(k^{2}-q^{2}\right) B \cos (p h+\alpha)+2 i k q A \cos (q h+\alpha)=0 \\
& \left(k^{2}-q^{2}\right) A \sin (q h+\alpha)+2 i k p B \sin (p h+\alpha)=0
\end{aligned}
$$

For compatability, the determinant of the coefficient in Eq. A.23 is set to zero,

$$
\left(k^{2}-q^{2}\right)^{2} \cos (p h+\alpha) \sin (q h+\alpha)+4 k^{2} p q \sin (p h+\alpha) \cos (q h+\alpha)=0
$$

By replacing $B$ in Eq. A.22 from Eq. A.23, the modified equation becomes

$$
\begin{aligned}
u_{1} & =q A\left[\cos \left(q x_{2}+\alpha\right)-\frac{2 k^{2}}{k^{2}-q^{2}} \frac{\cos (q h+\alpha)}{\cos (p h+\alpha)} \cos \left(p x_{2}+\alpha\right)\right. \\
u_{2} & =i k A\left[\sin \left(q x_{2}+\alpha\right)+\frac{2 p q}{k^{2}-q^{2}} \frac{\cos (q h+\alpha)}{\cos (p h+\alpha)} \sin \left(p x_{2}+\alpha\right)\right.
\end{aligned}
$$

Rewriting Eq. A.4 by replace $k$ by the phase velocity $V=\omega / k$, we get

$$
\begin{aligned}
& p^{2}=\omega^{2}\left(\frac{1}{V_{L}^{2}}-\frac{1}{V^{2}}\right) \\
& q^{2}=\omega^{2}\left(\frac{1}{V_{T}^{2}}-\frac{1}{V^{2}}\right)
\end{aligned}
$$

In case of a glass plate (thickness of $0.15 \mathrm{~mm}$ ) excited at frequency of $50 \mathrm{MHz}$ and $100 \mathrm{MHz}$, the lamb wave excited is in the high frequency region where $k h \gg 1$. Higher order antisymmetric modes are generated in the glass at these frequencies. For the A2 mode generated at $100 \mathrm{MHz}$, the phase velocity $(\mathrm{V})$ is such that $V_{T}<V<V_{L}$ and hence from Eq. A.26, $p=i k \chi$. The displacements for the antisymmetric mode are derived in the following. From Eq. A.24 for $\alpha=\pi / 2$, we get

$$
\left(k^{2}-q^{2}\right)^{2} \text { tanph }=-4 k^{2} \text { pqtanqh }
$$


with $p=i k \chi$,

$$
k\left(1-\frac{q^{2}}{k^{2}}\right)^{2} \tanh \chi h k=-4 \chi q \tan q h
$$

Since $V$ appraches close to $V_{T}, q \rightarrow 0$, hence for a large value of $k 10^{5}, q_{n} h \rightarrow$ $(2 n+1) \pi / 2$ where $\mathrm{n}=0,1,2$ etc. Substituting the value of $q_{n} h$ in Eq. A.25 gives the displacement for the mode $A_{n}$ as:

$$
\begin{array}{r}
u_{1} \cong\left(n+\frac{1}{2}\right) \frac{\pi}{h} A\left\{-\sin \left[(2 n+1) \pi \frac{x_{2}}{2 h}\right]+2(-1)^{n} \frac{\sinh \left(\chi k x_{2}\right)}{\sinh (\chi k h)}\right\} \\
u_{2} \cong i K A\left\{\cos \left[(2 n+1) \pi \frac{x_{2}}{2 h}\right]+(2 n+1)(-1)^{n} \frac{\pi \chi}{k h} \frac{\chi k x_{2}}{\chi h}\right\}
\end{array}
$$




\section{Appendix B}

\section{Transmission coefficient of a sound}

\section{wave from an arbitrary number of}

\section{layers}

Fig B.1 shows the sound wave incident from layer $\mathrm{n}+1$ with an incident angle of $\theta_{n+1}$ and unit amplitude. The wave undergoes reflection and the transmitted wave is converted to both longitudinal and transverse waves. In the lower space denoted by Layer 1, in the present case case refers to water, hence there is no transverse waves in Layer 1 . The waves contains a common factor $e^{i(\xi x-\omega t)}$ where,

$$
\xi=k_{n+1} \sin \theta_{n+1}=k_{i} \sin \theta_{i}=\kappa_{i} \sin \gamma_{i}, i=1,2 ., n .
$$

First, we consider the layer $\mathrm{n}$ and temporarily set the boundary of layer $\mathrm{n}$ and $\mathrm{n}-1$ as the origin of the coordinates. The expression for the potentials of the longitudinal and 


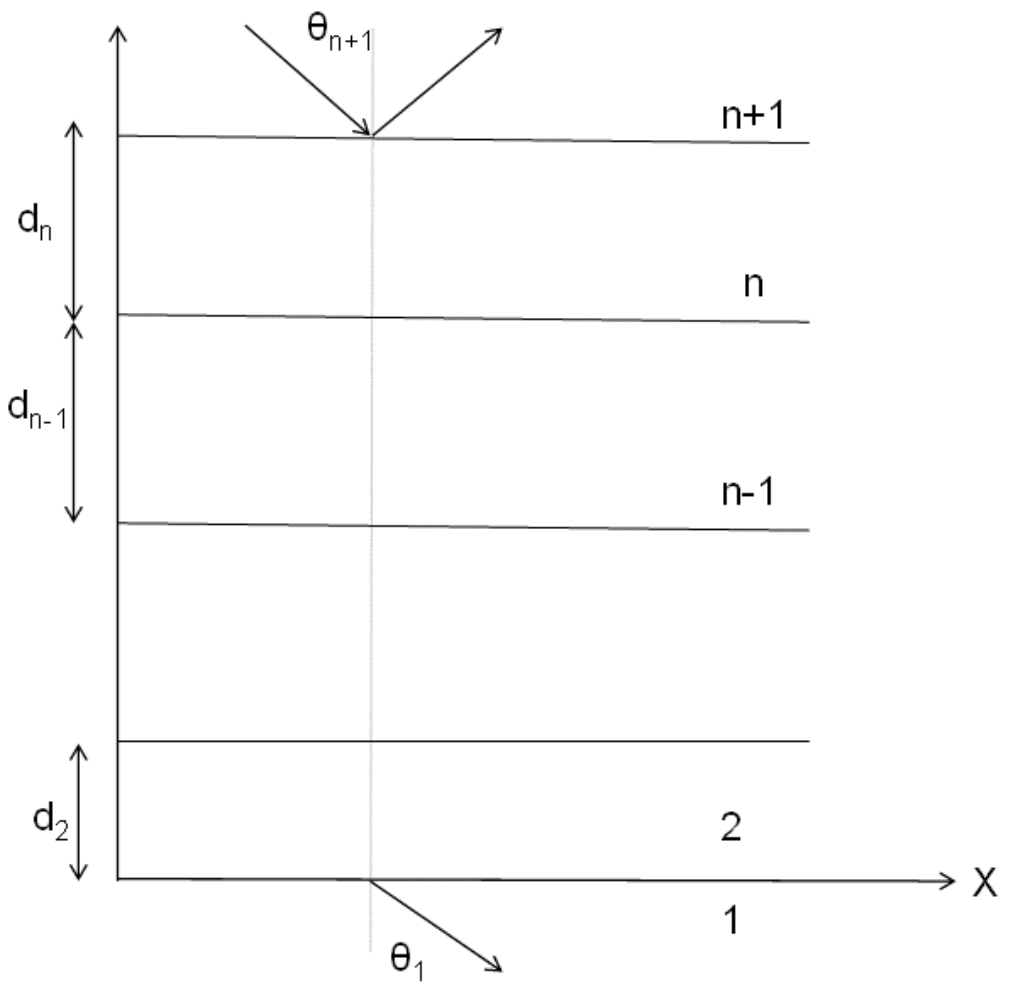

Figure B.1: Sound wave transmission through arbitrary number of layers

transverse waves as discussed in $\mathrm{A}$ are:

$$
\begin{gathered}
\phi=\phi^{\prime} e^{i \alpha z}+\phi^{\prime \prime} e^{-i \alpha x}, \alpha \equiv\left(k^{2}-\xi^{2}\right)^{1 / 2} \\
\psi=\psi^{\prime} e^{i \beta z}+\psi^{\prime \prime} e^{-i \beta x}, \beta \equiv\left(\kappa^{2}-\xi^{2}\right)^{1 / 2}
\end{gathered}
$$

The displacement can be expanded into various components using Eq. A.1 as:

$$
\begin{array}{r}
u_{x}=\partial_{x} \phi-\partial_{z} \psi \\
u_{y}=0 \\
u_{z}=\partial_{z} \phi+\partial_{x} \psi
\end{array}
$$


Similarily, using Eq. A.12 we can derive the normal stress $\left(T_{z z}=Z_{z}\right.$ and shear stress $T_{x z}=Z_{x}$ and $T_{y z}=Z_{y}$.

$$
\begin{array}{r}
Z_{z}=\lambda\left(\partial_{x} u_{x}+\partial_{z} u_{z}\right)+2 \mu \partial_{z} u_{z} \\
Z_{x}=\mu\left(\partial_{x} u_{z}+\partial_{z} u_{x}\right) \\
Z_{y}=\mu\left(\partial_{z} u_{y}\right)
\end{array}
$$

The quantities $u_{x}, u_{z}, Z_{z}, Z_{x}$ at the upper boundary of the layer $\mathrm{n}$ at $z=d$ is denoted by $u_{x}^{(n)}, u_{z}^{(n)}, Z_{x}^{(n)}, Z_{z}^{(n)}$. Using Eq. B.3 and Eq. B.4 gives:

$$
\begin{array}{r}
u_{x}^{(n)}=i \xi \cos P\left(\phi^{\prime}+\phi^{\prime \prime}\right)+\xi \sin P\left(\phi^{\prime}-\phi^{\prime \prime}\right)+i \xi \cos Q\left(\psi^{\prime}-\psi^{\prime \prime}\right)-\xi \sin Q\left(\psi^{\prime}+\psi^{\prime \prime}\right) \\
\text { where, } P=\alpha d, Q=\beta d
\end{array}
$$

Similarily expanding for the quantities $u_{z}^{(n)}, Z_{x}^{(n)}, Z_{z}^{(n)}$ and rearranging coefficients, we get the relation

$$
\begin{array}{r}
u_{z}^{(n)}=-\alpha \sin P\left(\phi^{\prime}+\phi^{\prime \prime}\right)-i \alpha \cos P\left(\phi^{\prime}-\phi^{\prime \prime}\right)+\xi \sin Q\left(\psi^{\prime}-\psi^{\prime \prime}\right)+i \xi \cos Q\left(\psi^{\prime}+\psi^{\prime \prime}\right) \\
Z_{z}^{(n)}=-\left(\lambda k^{2}+2 \mu \alpha^{2}\right) \cos P\left(\phi^{\prime}+\phi^{\prime \prime}\right)+i\left(\lambda k^{2}+2 \mu \alpha^{2}\right) \sin P\left(\phi^{\prime}-\phi^{\prime \prime}\right)+2 \mu \xi \beta \cos Q\left(\psi^{\prime}-\psi^{\prime \prime}\right) \\
-2 i \mu \xi \beta \sin Q\left(\psi^{\prime}+\psi^{\prime \prime}\right) \\
Z_{x}^{(n)}=2 i \mu \alpha \xi \sin P\left(\phi^{\prime}+\phi^{\prime \prime}\right)+2 \mu \alpha \xi \cos P\left(\phi^{\prime}-\phi^{\prime \prime}\right)+i \mu\left(\xi^{2}-\beta^{2}\right) \sin Q\left(\psi^{\prime}-\psi^{\prime \prime}\right)+ \\
\mu\left(\beta^{2}-\xi^{2}\right) \cos Q\left(\psi^{\prime}+\psi^{\prime \prime}\right)
\end{array}
$$

The displacement at the lower boundary of layer $n$ is obtained by setting $P=Q=0$.

$$
\begin{array}{r}
u_{x}^{(n-1)}=i \xi\left(\phi^{\prime}+\phi^{\prime \prime}\right)+i \xi\left(\psi^{\prime}-\psi^{\prime \prime}\right) \\
u_{z}^{(n-1)}=-i \alpha\left(\phi^{\prime}-\phi^{\prime \prime}\right)+i \xi\left(\psi^{\prime}+\psi^{\prime \prime}\right) \\
Z_{z}^{(n-1)}=-\left(\lambda k^{2}+2 \mu \alpha^{2}\right)\left(\phi^{\prime}+\phi^{\prime \prime}\right)+2 \mu \xi \beta\left(\psi^{\prime}-\psi^{\prime \prime}\right) \\
Z_{x}^{(n-1)}=2 \mu \alpha \xi\left(\phi^{\prime}-\phi^{\prime \prime}\right)+\mu\left(\beta^{2}-\xi^{2}\right)\left(\psi^{\prime}+\psi^{\prime \prime}\right)
\end{array}
$$


Combining Eq. B.5, Eq. B.7 and Eq. B.7, we get

$$
\left[\begin{array}{l}
u_{x}^{(n)} \\
u_{z}^{(n)} \\
Z_{z}^{(n)} \\
Z_{x}^{(n)}
\end{array}\right]=\left[\begin{array}{llll}
a_{11} & a_{12} & a_{13} & a_{14} \\
a_{21} & a_{22} & a_{23} & a_{24} \\
a_{31} & a_{32} & a_{33} & a_{34} \\
a_{41} & a_{42} & a_{43} & a_{44}
\end{array}\right]\left[\begin{array}{c}
u_{x}^{(n-1)} \\
u_{z}^{(n-1)} \\
Z_{z}^{(n-1)} \\
Z_{x}^{(n-1)}
\end{array}\right]
$$

where the elements of $a_{i} k(\mathrm{i}, \mathrm{k}=1,2,3,4)$ are given by

$$
\begin{array}{r}
a_{11}=2 \sin ^{2} \gamma \cos P+\cos 2 \gamma \cos Q \\
a_{12}=i(\tan \theta \cos 2 \gamma \sin P-\sin 2 \gamma \sin Q) \\
a_{13}=i \sin \theta(\cos Q-\cos P) / \omega \rho c \\
a_{14}=(\tan \theta \sin \gamma \sin P+\cos \gamma \sin Q) / \omega \rho b \\
a_{21}=i\left(2 \cot \theta \sin ^{2} \gamma \sin P-\tan \gamma \cos 2 \gamma \sin Q\right) \\
a_{22}=\cos 2 \gamma \cos P+2 \sin ^{2} \gamma \cos Q \\
a_{23}=(\cos \theta \sin P+\tan \gamma \sin \theta \sin Q) / \omega \rho c \\
a_{24}=i \sin \gamma(\cos Q-\cos P) / \omega \rho b \\
a_{31}=-2 i \omega \rho b \sin \gamma \cos 2 \gamma(\cos Q-\cos P) \\
a_{32}=-\omega \rho\left[(\cos 2 \gamma / \cos \theta) \sin P+4 b \cos \gamma \sin { }^{2} \gamma \sin Q\right] \\
a_{33}=\cos 2 \gamma \cos P+2 \sin { }^{2} \gamma \cos Q \\
a_{34}=i(\cos 2 \gamma t a n \theta \sin P-\sin 2 \gamma \sin Q) \\
a_{41}=-\omega \rho b^{2}\left[(4 / c) \cos \theta \sin ^{2} \gamma \sin P+(\cos 2 \gamma / b \cos \gamma) \sin Q\right] \\
a_{42}=-2 i \omega \rho b^{2} \sin \theta \cos 2 \gamma(\cos Q-\cos P) / c \\
a_{43}=i b^{2}\left[\left(\sin 2 \theta / c^{2}\right) \sin P-\left(\cos 2 \gamma / b^{2}\right) t a n \gamma \sin Q\right] \\
a_{44}=2 \sin { }^{2} \gamma \cos P+\cos 2 \gamma \cos Q
\end{array}
$$


Following the methods of successive application of Eq. B.8 we can write the result in the form:

$$
\left[\begin{array}{l}
u_{x}^{(n)} \\
u_{z}^{(n)} \\
Z_{z}^{(n)} \\
Z_{x}^{(n)}
\end{array}\right]=\left[\begin{array}{llll}
A_{11} & A_{12} & A_{13} & A_{14} \\
A_{21} & A_{22} & A_{23} & A_{24} \\
A_{31} & A_{32} & A_{33} & A_{34} \\
A_{41} & A_{42} & A_{43} & A_{44}
\end{array}\right]\left[\begin{array}{l}
u_{x}^{(1)} \\
u_{z}^{(1)} \\
Z_{z}^{(1)} \\
Z_{x}^{(1)}
\end{array}\right]
$$

where the matrix $A$ in Eq. B.10 is the product of matrices of a i.e, $A=a^{(n-1)} . . a^{(3)} a^{(2)}$. We represent the sum of layer thicknesses in B.1 by $H=\left(d_{2}+d_{3}+\ldots d_{n}\right)$. Considering only presence of longitudinal wave in the layer $n+1$ constituted by the liquid, the total field can be represented as

$$
\phi_{n+1}=e^{-i \alpha_{n+1}(z-H)}+V e^{i \alpha_{n+1}(z-H)}
$$

where $\alpha_{n+1}=k_{n+1} \cos \theta_{n+1}$ and $\mathrm{V}$ is the reflection coefficient. Considering that at the lower space, i.e at $n=1$, the medium is constituted by liquid, the potential consists of longitudinal waves represented as

$$
\phi_{1}=W e^{-i k_{1} z \cos \theta_{1}}
$$

where $\mathrm{W}$ represents the transmission coefficient of the longitudinal wave in medium 1. The coefficients $V$ and $W$ can be found from the conditions on the boundaries of media $n$ and $n+1$, where

$$
z=H, u_{z}^{(n+1)}=u_{z}^{(n)}, Z_{z}^{(n+1)}=Z_{z}^{n}, Z_{x}^{(n+1)}=Z_{x}^{n}=0
$$


Substituting the value of $u_{z}^{(n)}, Z_{z}^{(n)}$ and $Z_{x}^{(n)}$ from Eq. B.10, we get

$$
\begin{aligned}
u_{z}^{(n+1)} & =A_{21} u_{x}^{(1)}+A_{22} u_{z}^{(1)}+A_{23} Z_{z}^{(1)}+A_{24} Z_{x}^{(1)} \\
Z_{z}^{(n+1)} & =A_{31} u_{x}^{(1)}+A_{32} u_{z}^{(1)}+A_{33} Z_{z}^{(1)}+A_{34} Z_{x}^{(1)} \\
0 & =A_{41} u_{x}^{(1)}+A_{42} u_{z}^{(1)}+A_{43} Z_{z}^{(1)}+A_{44} Z_{x}^{(1)}
\end{aligned}
$$

where the values of $u_{z}^{(n+1)}$ and $Z_{z}^{(n+1)}$ are taken from $z=H$, while $u_{x}^{(1)}, . . Z_{x}^{(1)}$ from $z=0$. Replacing $u_{x}^{(1)}$ in the values of $u_{z}^{(n+1)}$ and $Z_{z}^{(n+1)}$ by substituting for the value of $u_{x}^{(1)}$ from last of Eq. B.14, we then have

$$
\begin{gathered}
u_{z}^{(n+1)}=M_{22} u_{z}^{(1)}+M_{23} Z_{z}^{(1)}+M_{24} Z_{x}^{(1)} \\
Z_{z}^{(n+1)}=M_{32} u_{Z}^{(1)}+M_{3} 3 Z_{z}^{(1)}+M_{34} Z_{x}^{(1)}
\end{gathered}
$$

where, $M_{i k}=A_{i k}-A_{i 1} A_{4 k} / A_{41}, i, k=2,3,4$ Next we obtain the expression for the various quantities at $\mathrm{z}=\mathrm{H}$ and $\mathrm{z}=0$. At $\mathrm{z}=\mathrm{H}$, we obtain $u_{z}^{(n+1)}$ and $Z_{z}^{(n+1)}$ by using Eq. B.3, Eq. B.4 and substituting the value of $\phi_{n+1}$ from Eq. B.11.

$$
\begin{array}{r}
u_{z}^{(n+1)}=i \alpha_{n+1}(V-1) \\
Z_{z}^{(n+1)}=-\omega^{2} \rho_{n+1}(1+V)
\end{array}
$$

Similarily at $z=0$, we use the form of $\phi_{1}$ in Eq. B.12 to derive the following values:

$$
\begin{array}{r}
u_{z}^{(1)}=i\left(-\alpha_{1} W\right) \\
u_{x}^{(1)}=i(\xi W) \\
Z_{z}^{(1)}=-\omega^{2} \rho_{1} W \\
Z_{x}^{(1)}=0
\end{array}
$$

From Eq. B.16 we find that the reflection coefficient $\mathrm{V}$ can be represented as:

$$
V=\frac{Z_{i n}-Z_{n+1}}{Z_{i n}+Z_{n+1}}
$$


where

$$
Z_{\text {in }}=\frac{i Z_{z}^{(n+1)}}{\omega u_{z}^{(n+1)}}, \quad Z_{n+1}=\rho_{n+1} c_{n+1} / \cos \left(\theta_{n+1}\right)
$$

By using Eq. B.17 in Eq. B.15 and substituting in Eq. B.19, the input impedance of the set of layers $Z_{i n}$ is written as:

$$
Z_{\text {in }}=(i / \omega)\left[\left(M_{32}-i \omega Z_{1} M_{33}\right) /\left(M_{22}-i \omega Z_{1} M_{23}\right)\right]
$$

We next derive the equations for the transmission coefficient $W$. Using the first equation of Eq. B.14, and substituting for the value of $u_{x}^{(1)}, u_{z}^{(1)}, Z_{z}^{(1)}, Z_{x}^{(1)}$, we get the following:

$$
W=\frac{i \alpha_{n+1}(V-1)}{i \xi A_{21}-i \alpha_{1} A_{22}-\omega^{2} \rho_{1} A_{23}}
$$

Replacing the value of $\mathrm{V}$ from Eq. B.18, gives

$$
W=\left(\frac{2 \alpha_{1} Z_{n+1}}{Z_{i n}+Z_{n+1}}\right)\left(\frac{1}{-\xi A_{21}+\alpha_{1} A_{22}-i \omega^{2} \rho_{1} A_{23}}\right)
$$

On applying the value of $Z_{\text {in }}$ from Eq. B.20 in Eq. B.22 and rearranging we get the following:

$$
\frac{\rho_{1}}{\rho_{n+1}} W=\frac{-2 i \omega Z_{1} \alpha_{1}\left[M_{22}-i \omega Z_{1} M_{23}\right]}{\left[\left(M_{32}-i \omega Z_{1} M_{33}\right)-\left(i \omega M_{22}+Z_{1} \omega^{2} M_{23}\right) Z_{n+1}\right]\left[-\xi A_{21}+\alpha_{1} A_{22}-i \omega^{2} \rho_{1} A_{23}\right]}
$$

where $Z_{1}=\omega \rho_{1} / \alpha_{1}$ On substituting the value of $M_{i k}$ in the term $\alpha_{1}\left[M_{22}-i \omega Z_{1} M_{23}\right]$ of Eq. B.23 in terms of the variable $A$ as discussed earlier, we can prove that $\alpha_{1}\left[M_{22}-\right.$ $\left.i \omega Z_{1} M_{23}\right]=\left[-\xi A_{21}+\alpha_{1} A_{22}-i \omega^{2} \rho_{1} A_{23}\right]$. Using these findings, the transmission coefficient $\mathrm{W}$ is defined as:

$$
\frac{\rho_{1}}{\rho_{n+1}} W=\frac{-2 i \omega Z_{1}}{\left(M_{32}-i \omega Z_{1} M_{33}\right)-\left(i \omega M_{22}+Z_{1} \omega^{2} M_{23}\right) Z_{n+1}}
$$


We write the quantities in Eq. B.24 in the form:

$$
\omega M_{23}=1 / Z_{1} N, \quad M_{22}+M_{33}=2 M / N, \quad M_{32}=\omega Z_{1}\left(M^{2}-N^{2}\right) / N \quad \text { (Eq. B.25) }
$$

where

$$
\begin{array}{r}
M=\left(Z_{2} / Z_{1}\right) \cos ^{2} 2 \gamma_{2} \cot P+\left(Z_{2 t} / Z_{1}\right) \sin ^{2} 2 \gamma_{2} \cot Q \\
N=Z_{2} \cos ^{2}\left(2 \gamma_{2}\right) / Z_{1} \sin P+Z_{2 t} \sin ^{2}\left(2 \gamma_{2}\right) / Z_{1} \sin Q \\
P=k_{2}(h / 2) \cos \theta_{2} \\
Q=\kappa(h / 2) \cos \gamma_{2}
\end{array}
$$

Thus, the transmission coefficient is written in the form:

$$
W=2 N /\left[2 M+i\left(M^{2}-N^{2}-1\right)\right]
$$




\section{Publication}

\section{Conference Papers}

(i) R. Kishor, Y. Zheng, W. Zhenfeng, and T. T. Lim, "FEM modelling of a SAW microfluidic sensor based on the photoacoustic effect" accepted at IEEE Int. UItrason. Symp., Tours, France,2016.

(ii) R. Kishor and Y. Zheng, "Surface acoustic wave RF sensing and actuation for lab-on-a-chip platforms", accepted at IEEE MTT-S International Microwave Workshop Series on Advanced Materials and Processes for RF and THz Applications (IMWS-AMP), China, 2016.

\section{Journal Papers}

(i) R. Kishor, Y. P. Seah, Y. J. Zheng, H. M. Xia, Z. F. Wang, H. J. Lu, et al., "Characterization of an acoustically coupled multilayered microfluidic platform on SAW substrate using mixing phenomena," Sensors and Actuators A: Physical, vol. 233, pp. 360-367, 2015

(ii) R. Kishor, F. Gao, S. Sreejith, X. Feng, Y. P. Seah, Z. Wang, et al., "Photoacoustic induced surface acoustic wave sensor for concurrent opto-mechanical microflu- 
idic sensing of dyes and plasmonic nanoparticles," RSC Advance, vol. 6, pp. 50238-50244, 2016.

(iii) R. Kishor, Ma Z.C., S. Sreejith, Y.P. Seah, Ai Y, Z.F. Wang, Lim T.T. and Y.J. Zheng, Integrated microfluidic system for real-time separation and detection of particles on a single piezoelectric substrate, Sensors and Actuators B:Chemical, (under revision).

(iv) R. Kishor, Y.P. Seah, Z.F. Wang, Lim T.T. and Y.J. Zheng, A compact separation and sensing platform for microparticles using othogonal surface acoustic wave devices, Lab on a chip, (to be submitted).

(v) R. Kishor,S. Sreejith, Y.P. Seah, Z.F. Wang, Lim T.T. and Y.J. Zheng, A SAW-PA integrated microfluidic sensing platform for detecting circulating tumor cells, (In preparation). 


\section{Bibliography}

[1] S. C. Terry, J. H. Jerman, and J. B. Angell. A gas chromatographic air analyzer fabricated on a silicon wafer. EEE Trans. Electron Devices, 26(12):1880-1886, 1979.

[2] A. Manz, N. Graber, and H.M. Widmer. Miniaturized total chemical analysis systems: A novel concept for chemical sensing. Sensors and Actuators B: Chemical, 1(1):244 - 248, 1990.

[3] Yanju Wang, Wei-Yu Lin, Kan Liu, Rachel J. Lin, Matthias Selke, Hartmuth C. Kolb, Nangang Zhang, Xing-Zhong Zhao, Michael E. Phelps, Clifton K. F. Shen, Kym F. Faull, and Hsian-Rong Tseng. An integrated microfluidic device for largescale in situ click chemistry screening. Lab Chip, 9:2281-2285, 2009.

[4] Rahim Esfandyarpour, Matthew J. DiDonato, Yuxin Yang, Naside Gozde Durmus, James S. Harris, and Ronald W. Davis. Multifunctional, inexpensive, and reusable nanoparticle-printed biochip for cell manipulation and diagnosis. Proc. Natl. Acad. Sci. U.S.A., 114(8):E1306-E1315, 2017. 10.1073/pnas.1621318114.

[5] J. G. E. Gardeniers and A. van den Berg. Lab-on-a-chip systems for biomedical and environmental monitoring. Analytical and Bioanalytical Chemistry, 378(7):1700-1703, 2004.

[6] Curtis D. Chin, Vincent Linder, and Samuel K. Sia. Commercialization of microfluidic point-of-care diagnostic devices. Lab Chip, 12(12):2118-2134, 2012. 
[7] Mazher Iqbal Mohammed, Steven Haswell, and lan Gibson. Lab-on-a-chip or chip-in-a-lab: Challenges of commercialization lost in translation. Procedia Technology, 20:54-59, 2015.

[8] George M. Whitesides. The origins and the future of microfluidics. Nature, 442(7101):368-373, 2006. 10.1038/nature05058.

[9] Jaime Castillo-León and Winnie E. Svendsen. Lab-on-a-Chip Devices and Micro-Total Analysis Systems A Practical Guide. 2015.

[10] Y. C. Lim, A. Z. Kouzani, and W. Duan. Lab-on-a-chip: a component view. Microsystem Technologies, 16(12):1995-2015, 2010.

[11] Jan G. Smits. Piezoelectric micropump with three valves working peristaltically. Sens. Actuators A, 21(1-3):203-206, 1990.

[12] D. J. Laser and J. G. Santiago. A review of micropumps. J. Micromech. Microeng., 14(6):R35, 2004.

[13] Ling-Sheng Jang, Yuan-Jie Li, Sung-Ju Lin, Yi-Chu Hsu, Wu-Sung Yao, MiChing Tsai, and Ching-Cheng Hou. A stand-alone peristaltic micropump based on piezoelectric actuation. Biomed Microdevices, 9(2):185-194, 2007.

[14] William H. Grover, Robin H. C. Ivester, Erik C. Jensen, and Richard A. Mathies. Development and multiplexed control of latching pneumatic valves using microfluidic logical structures. Lab Chip, 6(5):623-631, 2006.

[15] Kameel Abi-Samra, Liviu Clime, Ling Kong, Robert Gorkin, Tae-Hyeong Kim, Yoon-Kyoung Cho, and Marc Madou. Thermo-pneumatic pumping in centrifugal microfluidic platforms. Microfluidics and Nanofluidics, 11(5):643-652, 2011.

[16] Yu Zhou and Farid Amirouche. An electromagnetically-actuated all-pdms valveless micropump for drug delivery. Micromachines, 2(3):345, 2011.

[17] Machauf Andrew, Nemirovsky Yael, and Dinnar Uri. A membrane micropump electrostatically actuated across the working fluid. Journal of Micromechanics and Microengineering, 15(12):2309, 2005. (Prochaska).

[18] Aaron R. Wheeler. Putting electrowetting to work. Science, 322(5901):539-540, 
2008.

[19] Torsten Gerlach and Helmut Wurmus. Working principle and performance of the dynamic micropump. Sensors and Actuators A: Physical, 50(1-2):135-140, 1995.

[20] Chunsun Zhang, Da Xing, and Yuyuan Li. Micropumps, microvalves, and micromixers within pcr microfluidic chips: Advances and trends. Biotechnology Advances, 25(5):483-514, 2007.

[21] Christopher J. Easley, James M. Karlinsey, Joan M. Bienvenue, Lindsay A. Legendre, Michael G. Roper, Sanford H. Feldman, Molly A. Hughes, Erik L. Hewlett, Tod J. Merkel, Jerome P. Ferrance, and James P. Landers. A fully integrated microfluidic genetic analysis system with sample-in-answer-out capability. Proceedings of the National Academy of Sciences, 103(51):19272-19277, 2006.

[22] J. Steigert, M. Grumann, T. Brenner, K. Mittenbühler, T. Nann, J. Rühe, I. Moser, S. Haeberle, L. Riegger, J. Riegler, W. Bessler, R. Zengerle, and J. Ducrée. Integrated sample preparation, reaction, and detection on a high-frequency centrifugal microfluidic platform. JALA: Journal of the Association for Laboratory Automation, 10(5):331-341, 2005.

[23] Sungyoung Choi, Seungjeong Song, Chulhee Choi, and Je-Kyun Park. Continuous blood cell separation by hydrophoretic filtration. Lab Chip, 7(11):1532-1538, 2007.

[24] Arvind A. Bhagwat, Jitu Patel, Trina Chua, Audrey Chan, Saúl Ruiz Cruz, and Gustavo A. González Aguilar. Detection of Salmonella Species in Foodstuffs, pages 33-43. Humana Press, Totowa, NJ, 2008.

[25] Nguyen Nam-Trung and Wu Zhigang. Micromixers-a review. J. Micromech. Microeng., 15(2):R1, 2005.

[26] Elmabruk A. Mansur, Mingxing Ye, Yundong Wang, and Youyuan Dai. A state-ofthe-art review of mixing in microfluidic mixers. Chin. J. Chem. Eng., 16(4):503516, 2008. 
[27] H. M. Xia, S. Y. M. Wan, C. Shu, and Y. T. Chew. Chaotic micromixers using two-layer crossing channels to exhibit fast mixing at low reynolds numbers. Lab Chip, 5(7):748-755, 2005.

[28] Chia-Yen Lee, Chin-Lung Chang, Yao-Nan Wang, and Lung-Ming Fu. Microfluidic mixing: A review. International Journal of Molecular Sciences, 12(5):32633287, 2011.

[29] Daniel Ahmed, Xiaole Mao, Jinjie Shi, Bala Krishna Juluri, and Tony Jun Huang. A millisecond micromixer via single-bubble-based acoustic streaming. Lab Chip, 9(18):2738-2741, 2009.

[30] Trung-Dung Luong, Vinh-Nguyen Phan, and Nam-Trung Nguyen. Highthroughput micromixers based on acoustic streaming induced by surface acoustic wave. Microfluidics and Nanofluidics, 10(3):619-625, 2011.

[31] Ahmed Ould El Moctar, Nadine Aubry, and John Batton. Electro-hydrodynamic micro-fluidic mixer. Lab Chip, 3(4):273-280, 2003.

[32] Yan Du, Zhiyi Zhang, ChaeHo Yim, Min Lin, and Xudong Cao. A simplified design of the staggered herringbone micromixer for practical applications. Biomicrofluidics, 4(2):024105, 2010.

[33] Zhiyi Zhang, ChaeHo Yim, Min Lin, and Xudong Cao. Quantitative characterization of micromixing simulation. Biomicrofluidics, 2(3):034104, 2008.

[34] Michele Campisi, Dino Accoto, Francesco Damiani, and Paolo Dario. A softlithographed chaotic electrokinetic micromixer for efficient chemical reactions in lab-on-chips. Journal of Micro-Nano Mechatronics, 5(3):69-76, 2009.

[35] J. Deval, P. Tabeling, and Ho Chih-Ming. A dielectrophoretic chaotic mixer. In Technical Digest. MEMS 2002 IEEE International Conference. Fifteenth IEEE International Conference on Micro Electro Mechanical Systems (Cat. No.02CH37266), pages 36-39.

[36] Yiou Wang, Jiang Zhe, Benjamin T. F. Chung, and Prashanta Dutta. A rapid magnetic particle driven micromixer. Microfluidics and Nanofluidics, 4(5):375- 
389, 2008.

[37] Amy N. Hellman, Kaustubh R. Rau, Helen H. Yoon, Stephanie Bae, James F. Palmer, K. Scott Phillips, Nancy L. Allbritton, and Vasan Venugopalan. Laserinduced mixing in microfluidic channels. Analytical Chemistry, 79(12):44844492, 2007.

[38] Po-Hsun Huang, Yuliang Xie, Daniel Ahmed, Joseph Rufo, Nitesh Nama, Yuchao Chen, Chung Yu Chan, and Tony Jun Huang. An acoustofluidic micromixer based on oscillating sidewall sharp-edges. Lab Chip, 13(19):38473852, 2013. 23896797[pmid] Lab Chip.

[39] Orbay Sinem, Ozcelik Adem, Lata James, Kaynak Murat, Wu Mengxi, and Huang Tony Jun. Mixing high-viscosity fluids via acoustically driven bubbles. Journal of Micromechanics and Microengineering, 27(1):015008, 2017.

[40] Daniel Ahmed, Xiaole Mao, Bala Krishna Juluri, and Tony Jun Huang. A fast microfluidic mixer based on acoustically driven sidewall-trapped microbubbles. Microfluidics and Nanofluidics, 7(5):727, 2009.

[41] P. Sajeesh and Ashis Kumar Sen. Particle separation and sorting in microfluidic devices: a review. Microfluidics and Nanofluidics, 17(1):1-52, 2014.

[42] Mehmet Toner and Daniel Irimia. Blood-on-a-chip. Annual Review of Biomedical Engineering, 7(1):77-103, 2005.

[43] Petra S. Dittrich and Andreas Manz. Lab-on-a-chip: microfluidics in drug discovery. Nat Rev Drug Discov, 5(3):210-218, 2006.

[44] Darwin R. Reyes, Dimitri lossifidis, Pierre-Alain Auroux, and Andreas Manz. Micro total analysis systems. 1. introduction, theory, and technology. Analytical Chemistry, 74(12):2623-2636, 2002.

[45] Peng Li, Zhangming Mao, Zhangli Peng, Lanlan Zhou, Yuchao Chen, Po-Hsun Huang, Cristina I. Truica, Joseph J. Drabick, Wafik S. El-Deiry, Ming Dao, Subra Suresh, and Tony Jun Huang. Acoustic separation of circulating tumor cells. Proceedings of the National Academy of Sciences of the United States of America, 
112(16):4970-4975, 2015.

[46] Marie Podenphant, Neil Ashley, Kamila Koprowska, Kalim U. Mir, Maksim ZaIkovskij, Brian Bilenberg, Walter Bodmer, Anders Kristensen, and Rodolphe Marie. Separation of cancer cells from white blood cells by pinched flow fractionation. Lab Chip, 15(24):4598-4606, 2015.

[47] Palaniappan Sethu, Aaron Sin, and Mehmet Toner. Microfluidic diffusive filter for apheresis (leukapheresis). Lab Chip, 6(1):83-89, 2006.

[48] Barbara Roda, Pierluigi Reschiglian, Andrea Zattoni, Francesco Alviano, Giacomo Lanzoni, Roberta Costa, Arianna Di Carlo, Cosetta Marchionni, Michele Franchina, Laura Bonsi, and Gian Paolo Bagnara. A tag-less method of sorting stem cells from clinical specimens and separating mesenchymal from epithelial progenitor cells. Cytometry Part B: Clinical Cytometry, 76B(4):285-290, 2009.

[49] C. Wyatt Shields Iv, Catherine D. Reyes, and Gabriel P. Lopez. Microfluidic cell sorting: a review of the advances in the separation of cells from debulking to rare cell isolation. Lab Chip, 15(5):1230-1249, 2015.

[50] Lee Gi-Hun, Kim Sung-Hwan, Ahn Kihoon, Lee Sang-Hoon, and Park Joong Yull. Separation and sorting of cells in microsystems using physical principles. Journal of Micromechanics and Microengineering, 26(1):013003, 2016.

[51] Daniel R. Gossett, Westbrook M. Weaver, Albert J. Mach, Soojung Claire Hur, Henry Tat Kwong Tse, Wonhee Lee, Hamed Amini, and Dino Di Carlo. Labelfree cell separation and sorting in microfluidic systems. Analytical and Bioanalytical Chemistry, 397(8):3249-3267, 2010.

[52] Xiaoyun Ding, Zhangli Peng, Sz-Chin Steven Lin, Michela Geri, Sixing Li, Peng $\mathrm{Li}$, Yuchao Chen, Ming Dao, Subra Suresh, and Tony Jun Huang. Cell separation using tilted-angle standing surface acoustic waves. Proceedings of the National Academy of Sciences, 111(36):12992-12997, 2014.

[53] Ghulam Destgeer, Byung Hang Ha, Jin Ho Jung, and Hyung Jin Sung. Submicron separation of microspheres via travelling surface acoustic waves. Lab 
Chip, 14(24):4665-4672, 2014.

[54] R. Huang, T. A. Barber, M. A. Schmidt, R. G. Tompkins, M. Toner, D. W. Bianchi, R. Kapur, and W. L. Flejter. A microfluidics approach for the isolation of nucleated red blood cells (nrbcs) from the peripheral blood of pregnant women. Prenatal Diagnosis, 28(10):892-899, 2008.

[55] Andreas Lenshof, Asilah Ahmad-Tajudin, Kerstin Järås, Ann-Margret SwärdNilsson, Lena Åberg, György Marko-Varga, Johan Malm, Hans Lilja, and Thomas Laurell. Acoustic whole blood plasmapheresis chip for prostate specific antigen microarray diagnostics. Analytical Chemistry, 81(15):6030-6037, 2009.

[56] David J. Collins, Tuncay Alan, and Adrian Neild. Particle separation using virtual deterministic lateral displacement (vdld). Lab Chip, 14(9):1595-1603, 2014.

[57] Joo H. Kang, Silva Krause, Heather Tobin, Akiko Mammoto, Mathumai Kanapathipillai, and Donald E. Ingber. A combined micromagnetic-microfluidic device for rapid capture and culture of rare circulating tumor cells. Lab Chip, 12(12):2175-2181, 2012.

[58] I. Fang Cheng, Victoria E. Froude, Yingxi Zhu, Hsueh-Chia Chang, and HsienChang Chang. A continuous high-throughput bioparticle sorter based on $3 d$ traveling-wave dielectrophoresis. Lab Chip, 9(22):3193-3201, 2009.

[59] M. P. MacDonald, G. C. Spalding, and K. Dholakia. Microfluidic sorting in an optical lattice. Nature, 426(6965):421-424, 2003.

[60] Xiaolin Wang, Shuxun Chen, Marco Kong, Zuankai Wang, Kevin D. Costa, Ronald A. Li, and Dong Sun. Enhanced cell sorting and manipulation with combined optical tweezer and microfluidic chip technologies. Lab Chip, 11(21):3656-3662, 2011.

[61] Frank B. Myers and Luke P. Lee. Innovations in optical microfluidic technologies for point-of-care diagnostics. Lab Chip, 8(12):2015-2031, 2008.

[62] Pamela N. Nge, Chad I. Rogers, and Adam T. Woolley. Advances in mi- 
crofluidic materials, functions, integration, and applications. Chemical Reviews, 113(4):2550-2583, 2013.

[63] Bambang Kuswandi, Nuriman, Jurriaan Huskens, and Willem Verboom. Optical sensing systems for microfluidic devices: A review. Analytica Chimica Acta, 601(2):141-155, 2007.

[64] Haider A. J. Al Lawati. Flow-based analysis using microfluidicschemiluminescence systems. Luminescence, 28(5):618-627, 2013.

[65] Jing Wu and Min Gu. Microfluidic sensing: state of the art fabrication and detection techniques. Journal of Biomedical Optics, 16(8):080901-080901-12, 2011.

[66] Andrea Pais, Ansuman Banerjee, David Klotzkin, and lan Papautsky. Highsensitivity, disposable lab-on-a-chip with thin-film organic electronics for fluorescence detection. Lab Chip, 8(5):794-800, 2008.

[67] A. Banerjee, A. Pais, I. Papautsky, and D. Klotzkin. A polarization isolation method for high-sensitivity, low-cost on-chip fluorescence detection for microfluidic lab-on-a-chip. IEEE Sensors Journal, 8(5):621-627, 2008.

[68] Lidija Malic and Andrew G. Kirk. Integrated miniaturized optical detection platform for fluorescence and absorption spectroscopy. Sensors and Actuators A: Physical, 135(2):515-524, 2007.

[69] Gihan Ryu, Jingsong Huang, Oliver Hofmann, Claire A. Walshe, Jasmine Y. Y. Sze, Gareth D. McClean, Alan Mosley, Simon J. Rattle, John C. deMello, Andrew J. deMello, and Donal D. C. Bradley. Highly sensitive fluorescence detection system for microfluidic lab-on-a-chip. Lab Chip, 11(9):1664-1670, 2011.

[70] Anders M. Jorgensen, Klaus B. Mogensen, Jörg P. Kutter, and Oliver Geschke. A biochemical microdevice with an integrated chemiluminescence detector. Sensors and Actuators B: Chemical, 90(1-3):15-21, 2003.

[71] L. Zhu, C. S. Lee, and D. L. DeVoe. Integrated microfluidic uv absorbance detector with attomol-level sensitivity for bsa. Lab Chip, 6(1):115-120, 2006. 
[72] Heidi Ottevaere, Sara Van Overmeire, Jorge Albero, Lukasz Nieradko, Gert Desmet, Christophe Gorecki, and Hugo Thienpont. Plastic light coupler for absorbance detection in silicon microfluidic channels. Microfluidics and Nanofluidics, 18(4):559-568, 2015.

[73] Fang Wei, Jianghua Wang, Wei Liao, Bernhard G. Zimmermann, David T. Wong, and Chih-Ming Ho. Electrochemical detection of low-copy number salivary rna based on specific signal amplification with a hairpin probe. Nucleic Acids Research, 36(11):e65-e65, 2008.

[74] Xianqiao Hu, Yuanyuan Dong, Qiaohong He, Hengwu Chen, and Zhiwei Zhu. Fabrication of a polystyrene microfluidic chip coupled to electrospray ionization mass spectrometry for protein analysis. Journal of Chromatography B, 990:96103, 2015.

[75] Kandammathe Valiyaveedu Sreekanth, Yunus Alapan, Mohamed ElKabbash, Efe Ilker, Michael Hinczewski, Umut A. Gurkan, Antonio De Luca, and Giuseppe Strangi. Extreme sensitivity biosensing platform based on hyperbolic metamaterials. Nat Mater, 15(6):621-627, 2016.

[76] J. L. Arlett, E. B. Myers, and M. L. Roukes. Comparative advantages of mechanical biosensors. Nat Nano, 6(4):203-215, 2011.

[77] S. T. Ten, U. Hashim, S. C. B. Gopinath, W. W. Liu, K. L. Foo, S. T. Sam, S. F. A. Rahman, C. H. Voon, and A. N. Nordin. Highly sensitive escherichia coli shear horizontal surface acoustic wave biosensor with silicon dioxide nanostructures. Biosensors and Bioelectronics.

[78] Hua-Lin Cai, Yi Yang, Xiao Chen, Mohammad Ali Mohammad, Tian-Xiang Ye, Cang-Ran Guo, Li-Ting Yi, Chang-Jian Zhou, Jing Liu, and Tian-Ling Ren. A third-order mode high frequency biosensor with atomic resolution. Biosensors and Bioelectronics, 71:261-268, 2015.

[79] A. Oseev, R. Lucklum, M. Zubtsov, M. P. Schmidt, and S. Hirsch. Saw based phononic crystal liquid sensor - periodic microfluidic channels approach. In 2016 
IEEE International Ultrasonics Symposium (IUS), pages 1-4.

[80] Kyu Hyun Kim, Gaurav Bahl, Wonsuk Lee, Jing Liu, Matthew Tomes, Xudong Fan, and Tal Carmon. Cavity optomechanics on a microfluidic resonator with water and viscous liquids. Light Sci Appl, 2:e110, 2013.

[81] H. M. Lai and K. Young. Theory of the pulsed optoacoustic technique. The Journal of the Acoustical Society of America, 72(6):2000-2007, 1982.

[82] J. Uotila, V. Koskinen, and J. Kauppinen. Selective differential photoacoustic method for trace gas analysis. Vibrational Spectroscopy, 38(1-2):3-9, 2005.

[83] V. Koskinen, J. Fonsen, J. Kauppinen, and I. Kauppinen. Extremely sensitive trace gas analysis with modern photoacoustic spectroscopy. Vibrational Spectroscopy, 42(2):239-242, 2006.

[84] Chengzhong Cai, Kai A. Carey, Dmitry A. Nedosekin, Yulian A. Menyaev, Mustafa Sarimollaoglu, Ekaterina I. Galanzha, Jason S. Stumhofer, and Vladimir P. Zharov. In vivo photoacoustic flow cytometry for early malaria diagnosis. Cytometry Part A, 89(6):531-542, 2016.

[85] Thomas Schmid. Photoacoustic spectroscopy for process analysis. Analytical and Bioanalytical Chemistry, 384(5):1071-1086, 2006.

[86] G. J. Diebold and P. J. Westervelt. The photoacoustic effect generated by a spherical droplet in a fluid. The Journal of the Acoustical Society of America, 84(6):2245-2251, 1988.

[87] L. V. Wang. Tutorial on photoacoustic microscopy and computed tomography. IEEE Journal of Selected Topics in Quantum Electronics, 14(1):171-179, 2008.

[88] Jie Hui and Ji-Xin Cheng. Vibrational photoacoustic microscopy: Converting molecular vibration to mechanical for deep-tissue imaging, 2017.

[89] Colin Campbell. Surface acoustic wave devices for mobile and wireless communications. Academic Press, San Diego, 1998.

[90] David P. Morgan. Surface acoustic wave filters : with applications to electronic communications and signal processing. Academic Press, Amsterdam; London, 
2007.

[91] Supriyo Datta. Surface acoustic wave devices. Prentice-Hall, Englewood Cliffs, N.J., 1986.

[92] A. Karabutov Aleksander. Laser excitation of surface acoustic waves: a new direction in opto-acoustic spectroscopy of a solid. Soviet Physics Uspekhi, 28(11):1042, 1985.

[93] H. L. Bertoni and T. Tamir. Characteristics of wedge transducers for acoustic surface waves. IEEE Transactions on Sonics and Ultrasonics, 22(6):415-420, 1975.

[94] R. F. Mitchell and D. W. Parker. Synthesis of acoustic-surface-wave filters using double electrodes. Electronics Letters, 10(24):512-512, 1974.

[95] C. K. Campbell. Applications of surface acoustic and shallow bulk acoustic wave devices. Proceedings of the IEEE, 77(10):1453-1484, 1989.

[96] V. Plessky and M. Methfessel. Saw transformer-filter. In 2016 IEEE International Ultrasonics Symposium (IUS), pages 1-4.

[97] Xiaoyun Ding, Peng Li, Sz-Chin Steven Lin, Zackary S. Stratton, Nitesh Nama, Feng Guo, Daniel Slotcavage, Xiaole Mao, Jinjie Shi, Francesco Costanzo, and Tony Jun Huang. Surface acoustic wave microfluidics. Lab Chip, 13(18):36263649, 2013.

[98] Leslie Y. Yeo and James R. Friend. Surface acoustic wave microfluidics. Annual Review of Fluid Mechanics, 46(1):379-406, 2014.

[99] James Friend and Leslie Y. Yeo. Microscale acoustofluidics: Microfluidics driven via acoustics and ultrasonics. Reviews of Modern Physics, 83(2):647-704, 2011. RMP.

[100] Jinjie Shi, Hua Huang, Zak Stratton, Yiping Huang, and Tony Jun Huang. Continuous particle separation in a microfluidic channelvia standing surface acoustic waves (ssaw). Lab Chip, 9(23):3354-3359, 2009.

[101] Xiaoyun Ding, Sz-Chin Steven Lin, Brian Kiraly, Hongjun Yue, Sixing Li, I-Kao 
Chiang, Jinjie Shi, Stephen J. Benkovic, and Tony Jun Huang. On-chip manipulation of single microparticles, cells, and organisms using surface acoustic waves. Proceedings of the National Academy of Sciences, 109(28):1110511109, 2012.

[102] Myeong Chan Jo and Rasim Guldiken. Particle manipulation by phase-shifting of surface acoustic waves. Sensors and Actuators A: Physical, 207:39-42, 2014.

[103] Henrik Bruus. Acoustofluidics 7: The acoustic radiation force on small particles. Lab Chip, 12(6):1014-1021, 2012.

[104] Martin Gröschl. Ultrasonic separation of suspended particles - part i: Fundamentals. Acta Acustica united with Acustica, 84(3):432-447, 1998.

[105] C. C. W. Ruppel, L. Reindl, and R. Weigel. Saw devices and their wireless communications applications. IEEE Microwave Magazine, 3(2):65-71, 2002.

[106] Haiyan Li, James Friend, Leslie Yeo, Ayan Dasvarma, and Kathy Traianedes. Effect of surface acoustic waves on the viability, proliferation and differentiation of primary osteoblast-like cells. Biomicrofluidics, 3(3):034102, 2009.

[107] Ryan P. Hodgson, Tan Ming, L. Yeo, and J. Friend. Transmitting high power if acoustic radiation via fluid couplants into superstrates for microfluidics. Applied Physics Letters, 94(2):024102-024102-3, 2009.

[108] Rahul Kishor, Y.P. Seah, Y.J. Zheng, H.M. Xia, Z.F. Wang, H.J. Lu, and T.T. Lim. Characterization of an acoustically coupled multilayered microfluidic platform on \{SAW\} substrate using mixing phenomena. Sensors and Actuators A: Physical, 233:360 - 367, 2015.

[109] Yannyk Bourquin, Julien Reboud, Rab Wilson, and Jonathan M. Cooper. Tuneable surface acoustic waves for fluid and particle manipulations on disposable chips. Lab on a Chip, 10(15):1898-1901, 2010.

[110] Thomas Frommelt, Marcin Kostur, Melanie Wenzel-Schäfer, Peter Talkner, Peter Hänggi, and Achim Wixforth. Microfluidic mixing via acoustically driven chaotic 
advection. Physical Review Letters, 100(3):034502, 2008.

[111] Achim Wixforth. Acoustically driven programmable microfluidics for biological and chemical applications. Journal of the Association for Laboratory Automation, 11(6):399-405, 2006.

[112] Richard Shilton, Ming K. Tan, Leslie Y. Yeo, and James R. Friend. Particle concentration and mixing in microdrops driven by focused surface acoustic waves. Journal of Applied Physics, 104(1):014910, 2008.

[113] Richie J. Shilton, Leslie Y. Yeo, and James R. Friend. Quantification of surface acoustic wave induced chaotic mixing-flows in microfluidic wells. Sensors and Actuators B: Chemical, 160(1):1565-1572, 2011.

[114] Wei-Kuo Tseng, Jr-Lung Lin, Wang-Chou Sung, Shu-Hui Chen, and Gwo-Bin Lee. Active micro-mixers using surface acoustic waves on y-cut $128^{\circ}$ linbo3. Journal of Micromechanics and Microengineering, 16(3):539, 2006.

[115] K. Sritharan, C. J. Strobl, M. F. Schneider, A. Wixforth, and Z. Guttenberg. Acoustic mixing at low reynold's numbers. Applied Physics Letters, 88(5):054102, 2006.

[116] M. K. Tan, L. Y. Yeo, and J. R. Friend. Rapid fluid flow and mixing induced in microchannels using surface acoustic waves. EPL (Europhysics Letters), 87(4):47003, 2009.

[117] Trung-Dung Luong, Vinh-Nguyen Phan, and Nam-Trung Nguyen. Highthroughput micromixers based on acoustic streaming induced by surface acoustic wave. Microfluidics and Nanofluidics, 10(3):619-625, 2011.

[118] Linda Johansson, Johannes Enlund, Stefan Johansson, llia Katardjiev, and Ventsislav Yantchev. Surface acoustic wave induced particle manipulation in a pdms channel—principle concepts for continuous flow applications. Biomedical Microdevices, 14(2):279-289, 2012.

[119] Daniel Mark, Stefan Haeberle, Gunter Roth, Felix von Stetten, and Roland Zengerle. Microfluidic lab-on-a-chip platforms: requirements, characteristics 
and applications. Chemical Society Reviews, 39(3):1153-1182, 2010.

[120] Paul Yager, Gonzalo J. Domingo, and John Gerdes. Point-of-care diagnostics for global health. Annual Review of Biomedical Engineering, 10(1):107-144, 2008.

[121] Thirumalai Kannan. Finite Element Analysis of Surface Acoustic Wave Resonators. PhD thesis, 2006.

[122] H. M. Xia, Z. P. Wang, Y. X. Koh, and K. T. May. A microfluidic mixer with selfexcited 'turbulent' fluid motion for wide viscosity ratio applications. Lab on $a$ Chip, 10(13):1712-1716, 2010.

[123] Caroline A. Schneider, Wayne S. Rasband, and Kevin W. Eliceiri. Nih image to imagej: 25 years of image analysis. Nat Meth, 9(7):671-675, 2012.

[124] S. Shiokawa, Y. Matsui, and T. Ueda. Liquid streaming and droplet formation caused by leaky rayleigh waves. In IEEE Ultrasonics Symposium, volume 1, pages 643-646.

[125] Nguyen Nam-Trung and R. M. White. Acoustic streaming in micromachined flexural plate wave devices: numerical simulation and experimental verification. Ultrasonics, Ferroelectrics and Frequency Control, IEEE Transactions on, 47(6):1463-1471, 2000.

[126] Junhui Hu, Kentaro Nakamura, and Sadauki Ueha. Optimum operation conditions of an ultrasonic motor driving fluid directly. Japanese Journal of Applied Physics, 35(5S):3289, 1996.

[127] P. Brunet, M. Baudoin, O. Bou Matar, and F. Zoueshtiagh. Droplet displacements and oscillations induced by ultrasonic surface acoustic waves: A quantitative study. Physical Review E, 81(3):036315, 2010.

[128] J M M Pinkerton. The absorption of ultrasonic waves in liquids and its relation to molecular constitution. Proceedings of the Physical Society. Section B, 62(2):129, 1949.

[129] Lawrence E. Kinsler. Fundamentals of acoustics. Fourth edition, 2000. 
[130] Hyo Ung Li and Katsuo Negishi. Visualization of lamb mode patterns in a glass plate. Ultrasonics, 32(4):243-248, 1994.

[131] D. Royer and E. Dieulesaint. Elastic Waves in Solids II Generation, Acoustooptic Interaction, Applications. Advanced texts in physics,. Springer, Berlin ; New York, 2000.

[132] D. Royer and E. Dieulesaint. Elastic Waves in Solids I: Free and Guided Propagation. Advanced texts in physics,. Springer, Berlin ; New York, 2000.

[133] L. M. Brekhovskikh. Waves in layered media. Applied mathematics and mechanics. Academic Press, New York, 2d edition, 1980.

[134] Martín Chávez, Victor Sosa, and Ricardo Tsumura. Speed of sound in saturated pure water. The Journal of the Acoustical Society of America, 77(2):420-423, 1985.

[135] L.W.Tilton and J.K Taylor. Accurate representation of the refractivity and density of distilled water as a function of temperature. Journal of Research of the National Bureau of Standards, 18:205-214, 1937.

[136] D. Ciplys and R. Rimeika. Characterization of liquids using leaky surface acoustic waves in yx-litao3. In IEEE Ultrason. Symp. Proc., pages 1333-1336.

[137] T. Nomura, A. Saitoh, and Y. Horikoshi. Measurement of acoustic properties of liquid using liquid flow sh-saw sensor system. Sens. Actuators, B, 76(1-3):6973, 2001.

[138] Lars Egil Helseth. Simultaneous measurements of absorption spectrum and refractive index in a microfluidic system. Optics Express, 20(4):4653-4662, 2012.

[139] Ilpo Niskanen, Jukka Räty, and Kai-Erik Peiponen. Optical sensing of concentration and refractive index of pigments in a suspension. Applied Optics, 49(17):3428-3433, 2010.

[140] Emanuel Weber and Michael J. Vellekoop. Optofluidic micro-sensors for the determination of liquid concentrations. Lab Chip, 12(19):3754-3759, 2012.

[141] Hongbin Yu, Guangya Zhou, Fook Siong Chau, and Feiwen Lee. Phase- 
transmission-grating-based compact optofluidic refractometer. Optics Letters, 34(12):1753-1755, 2009.

[142] Camilo Guzmán, Henrik Flyvbjerg, Roland Köszali, Carole Ecoffet, László Forró, and Sylvia Jeney. In situ viscometry by optical trapping interferometry. Appl. Phys. Lett., 93(18):184102, 2008.

[143] Stephen Keen, Alison Yao, Jonathan Leach, Roberto Di Leonardo, Chris Saunter, Gordon Love, Jonathan Cooper, and Miles Padgett. Multipoint viscosity measurements in microfluidic channels using optical tweezers. Lab Chip, 9(14):2059-2062, 2009.

[144] Fei Gao, Xiaohua Feng, Yuanjin Zheng, and Claus Dieter Ohl. Photoacoustic resonance spectroscopy for biological tissue characterization. J. Biomed. Opt., 19(6):067006, 2014.

[145] Fei Gao, Xiaohua Feng, and Yuanjin Zheng. Photoacoustic phasoscopy supercontrast imaging. Appl. Phys. Lett., 104(21):213701, 2014.

[146] Lihong V. Wang and Song Hu. Photoacoustic tomography: In vivo imaging from organelles to organs. Science, 335(6075):1458-1462, 2012.

[147] Minghua Xu and Lihong V. Wang. Photoacoustic imaging in biomedicine. Rev. Sci. Instrum., 77(4):041101, 2006.

[148] Sivaramapanicker Sreejith, KizhumuriâĂĚP Divya, and Ayyappanpillai Ajayaghosh. A near-infrared squaraine dye as a latent ratiometric fluorophore for the detection of aminothiol content in blood plasma. Angewandte Chemie International Edition, 47(41):7883-7887, 2008.

[149] Sivaramapanicker Sreejith, James Joseph, Kim Truc Nguyen, Vadakke Matham Murukeshan, Sun Woh Lye, and Yanli Zhao. Graphene oxide wrapping of goldsilica core-shell nanohybrids for photoacoustic signal generation and bimodal imaging. ChemNanoMat, 1(1):39-45, 2015.

[150] Abhijit Biswas, Tao Wang, and Alexandru S. Biris. Single metal nanoparticle spectroscopy: optical characterization of individual nanosystems for biomedical 
applications. Nanoscale, 2(9):1560-1572, 2010.

[151] A A Karabutov. Laser excitation of surface acoustic waves: a new direction in opto-acoustic spectroscopy of a solid. SOV PHYS USPEKHI, 28(11):1042$1051,1985$.

[152] M Ochiai. Laser-induced surface acoustic wave technique for precise depth measurement of stress corrosion cracking. Journal of Physics: Conference Series, 278(1):012009, 2011.

[153] Rahul Kishor, Fei Gao, Sivaramapanicker Sreejith, Xiaohua Feng, Yen Peng Seah, Zhenfeng Wang, Mihaiela Corina Stuparu, Teik-Thye Lim, Xiaodong Chen, and Yuanjin Zheng. Photoacoustic induced surface acoustic wave sensor for concurrent opto-mechanical microfluidic sensing of dyes and plasmonic nanoparticles. RSC Adv., 6(55):50238-50244, 2016.

[154] R. Kishor, Zheng Yuanjin, Zhenfeng Wang, and T. T. Lim. Fem modelling of a saw microfluidic sensor based on the photoacoustic effect. In 2016 IEEE International Ultrasonics Symposium (IUS), pages 1-4, Sept 2016.

[155] J. Fraser, B. T. Khuri Yakub, and G. S. Kino. The design of efficient broadband wedge transducers. Appl. Phys. Lett., 32(11):698-700, 1978.

[156] Shinichi Nakagawa and Holger Schielzeth. Repeatability for gaussian and nongaussian data: a practical guide for biologists. Biological Reviews, 85(4):935956, 2010.

[157] Jing Wang, Tan Liu, Shuliang Jiao, Ruimin Chen, Qifa Zhou, K. Kirk Shung, Lihong V. Wang, and Hao F. Zhang. Saturation effect in functional photoacoustic imaging. Journal of Biomedical Optics, 15(2):021317-021317-5, 2010.

[158] Qingliang Zhao, Lin Li, Qian Li, Xia Jiang, Qiushi Ren, Xinyu Chai, and Chuanqing Zhou. Concentration dependence of optical clearing on the enhancement of laser-scanning optical-resolution photoacoustic microscopy imaging. J. Biomed. Opt., 19(3):036019-036019, 2014.

[159] Alessandro Feis, Cristina Gellini, Pier Remigio Salvi, and Maurizio Be- 
cucci. Photoacoustic excitation profiles of gold nanoparticles. Photoacoustics, 2(1):47-53, 2014.

[160] Jin-Woo Kim, Ekaterina I. Galanzha, Evgeny V. Shashkov, Hyung-Mo Moon, and Vladimir P. Zharov. Golden carbon nanotubes as multimodal photoacoustic and photothermal high-contrast molecular agents. Nat Nano, 4(10):688-694, 2009.

[161] Martín G. González, Xiangjiang Liu, Reinhard Niessner, and Christoph Haisch. Strong size-dependent photoacoustic effect on gold nanoparticles by laserinduced nanobubbles. Applied Physics Letters, 96(17):174104, 2010.

[162] Chenxu Yu and Joseph Irudayaraj. Multiplex biosensor using gold nanorods. Analytical Chemistry, 79(2):572-579, 2007.

[163] Prashant K. Jain, Kyeong Seok Lee, Ivan H. El-Sayed, and Mostafa A. ElSayed. Calculated absorption and scattering properties of gold nanoparticles of different size, shape, and composition: Applications in biological imaging and biomedicine. J Phys Chem B., 110(14):7238-7248, 2006.

[164] Eric M Strohm, Ivan Gorelikov, Naomi Matsuura, and Michael C Kolios. Modeling photoacoustic spectral features of micron-sized particles. Physics in Medicine and Biology, 59(19):5795, 2014.

[165] Kannan Thirumalai. Finite element analysis of surface acoustic wave resonators. Master's thesis, University of Saskatchewan, 2006.

[166] Oleg A. Godin. Sound transmission through water-air interfaces: new insights into an old problem. Contemporary Physics, 49(2):105-123, 2008.

[167] Taehwa Lee, Qiaochu Li, and L. Jay Guo. Out-coupling of longitudinal photoacoustic pulses by mitigating the phase cancellation. Scientific Reports, 6:21511, 2016.

[168] A Rona. The acoustic resonance of rectangular and cylindrical cavities. Journal of Algorithms \& Computational Technology, 1(3):329-356, 2007.

[169] Sivaramapanicker Sreejith, James Joseph, Manjing Lin, Nishanth Venugopal 
Menon, Parijat Borah, Hao Jun Ng, Yun Xian Loong, Yuejun Kang, Sidney Wing-Kwong Yu, and Yanli Zhao. Near-infrared squaraine dye encapsulated micelles for in vivo fluorescence and photoacoustic bimodal imaging. ACS Nano, 9(6):5695-5704, 2015.

[170] David Bell and Rosanna W. Peeling. Evaluation of rapid diagnostic tests: malaria. Nat Rev Micro, 4(9 suppl):S34-8, 2006.

[171] Onur Tokel, Umit Hakan Yildiz, Fatih Inci, Naside Gozde Durmus, Okan Oner Ekiz, Burak Turker, Can Cetin, Shruthi Rao, Kaushik Sridhar, Nalini Natarajan, Hadi Shafiee, Aykutlu Dana, and Utkan Demirci. Portable microfluidic integrated plasmonic platform for pathogen detection. Sci. Rep., 5:9152, 2015.

[172] Jeongan Choi, Miran Kang, and Jae Hee Jung. Integrated micro-optofluidic platform for real-time detection of airborne microorganisms. Sci. Rep., 5:15983, 2015.

[173] Tian Fook Kong, Weijian Ye, Weng Kung Peng, Han Wei Hou, Marcos, Peter Rainer Preiser, Nam-Trung Nguyen, and Jongyoon Han. Enhancing malaria diagnosis through microfluidic cell enrichment and magnetic resonance relaxometry detection. Sci. Rep., 5:11425, 2015.

[174] Achim Wixforth. Acoustically driven planar microfluidics. SUPERLATTICE MICROST, 33(5-6):389-396, 2003.

[175] David J. Collins, Citsabehsan Devendran, Zhichao Ma, Jia Wei Ng, Adrian Neild, and Ye Ai. Acoustic tweezers via sub-time-of-flight regime surface acoustic waves. Sci. Adv., 2(7), 2016.

[176] Priscilla R. Rogers, James R. Friend, and Leslie Y. Yeo. Exploitation of surface acoustic waves to drive size-dependent microparticle concentration within a droplet. Lab Chip, 10(21):2979-2985, 2010.

[177] Ghulam Destgeer, Hyunjun Cho, Byung Hang Ha, Jin Ho Jung, Jinsoo Park, and Hyung Jin Sung. Acoustofluidic particle manipulation inside a sessile droplet: four distinct regimes of particle concentration. Lab Chip, 16(4):660-667, 2016. 
[178] Julien Reboud, Craig Auchinvole, Christopher D. Syme, Rab Wilson, and Jonathan M. Cooper. Acoustically controlled enhancement of molecular sensing to assess oxidative stress in cells. Chem. Commun., 49(28):2918-2920, 2013.

[179] Michael J. Heller and András Guttman. Integrated microfabricated biodevices : advanced technology for genomics, drug discovery, bioanalysis, and clinical diagnostics. Marcel Dekker, Inc., New York, 2002.

[180] A. Holm, Q. Stürzer, Y. Xu, and R. Weigel. Investigation of surface acoustic waves on linbo3, quartz, and litao3 by laser probing. Microelectronic Engineering, 31(1):123 - 127, 1996.

[181] Peter Barkholt Muller, Rune Barnkob, Mads Jakob Herring Jensen, and Henrik Bruus. A numerical study of microparticle acoustophoresis driven by acoustic radiation forces and streaming-induced drag forces. Lab Chip, 12(22):46174627, 2012.

[182] Richard P. Hodges. Underwater acoustics : analysis, design and performance of sonar, 2013. 